University of Louisville

ThinkIR: The University of Louisville's Institutional Repository

Electronic Theses and Dissertations

$8-2020$

\title{
Genes involved in nitrogen assimilation in Ustilago maydis.
}

Sunita Khanal
University of Louisville

Follow this and additional works at: https://ir.library.louisville.edu/etd

Part of the Molecular Genetics Commons

\section{Recommended Citation}

Khanal, Sunita, "Genes involved in nitrogen assimilation in Ustilago maydis." (2020). Electronic Theses and Dissertations. Paper 3492.

https://doi.org/10.18297/etd/3492

This Doctoral Dissertation is brought to you for free and open access by ThinkIR: The University of Louisville's Institutional Repository. It has been accepted for inclusion in Electronic Theses and Dissertations by an authorized administrator of ThinkIR: The University of Louisville's Institutional Repository. This title appears here courtesy of the author, who has retained all other copyrights. For more information, please contact thinkir@louisville.edu. 
GENES INVOLVED IN NITROGEN ASSIMILATION IN USTILAGO MAYDIS

\author{
By \\ Sunita Khanal \\ B.Sc., Tribhuvan University, Nepal, 2010 \\ M.Sc., Tribhuvan University, Nepal, 2013 \\ M.S., University of Louisville, USA, 2017
}

\begin{abstract}
A Dissertation
Submitted to the Faculty of the

College of Arts and Sciences of the University of Louisville in Partial Fulfillment of the Requirements for the Degree of
\end{abstract}

Doctor of Philosophy in Biology

Department of Biology, Division of Molecular, Cellular and Developmental Biology, College of Arts and Sciences

University of Louisville, Louisville, Kentucky

August 2020 

GENES INVOLVED IN NITROGEN ASSIMILATION IN USTILAGO MAYDIS

By

Sunita Khanal

B.Sc., Tribhuvan University, Nepal, 2010

M.Sc., Tribhuvan University, Nepal, 2013

M.S., University of Louisville, USA, 2017

A Dissertation Approved on

July 30, 2020

By the following Dissertation Committee:

Dr. Michael H. Perlin, Principal Advisor

Dr. Cynthia Corbitt

Dr. Mark P. Running

Dr. David J. Schultz

Dr. Farrukh Aqil 


\section{ACKNOWLEDGEMENTS}

First and foremost, I would like to thank my mentor Dr. Michael H. Perlin for his support, patience, enthusiasm and motivation. His guidance has helped me throughout this journey from the shaping of the project to the writing of the dissertation. His constructive criticism, prompt response to any questions and true scientific wisdom have

enriched my growth as a student. Apart from being a great mentor, he is an extraordinary human being. He is humorous, friendly, full of joy and his nature of celebrating success made the lab environment lively. I would also like to thank the rest of my committee members Drs. Mark P. Running, David J. Schultz, Cynthia Corbitt and Farrukh Aqil for their valuable time, critical comments and constructive feedback. Their guidance, suggestions and insights have helped a lot for the progress of this project.

This project wouldn't have been completed without the support of all present and past Goat lab members. Thanks to Michael Cooper for his findings and the list of the potential genes to begin the project. I am thankful to Margaret Wallen and Lalu Krishna for helping me understand the Ustilago system and basic techniques involved in working with $U$. maydis. A big thanks to Swathi Kuppireddy for providing her input to help me troubleshoot any problems I come across, both at a personal level and in science. Even after their graduation, these doctorates have helped me grow and stay focused as I got closer to my dissertation completion. 
One of the greatest achievements in joining goat lab is having great lab colleagues and awesome friends. Hector Mendoza is a great human being and a great friend. He doesn't hesitate to help with anything asked for and has always helped me in completing experiments at odd lab hours. Also, many thanks to Ming-Chang Tsai (Nelson), Joseph P. Ham (JP), Roxanne Leiter (favorite) and William Beckerson who have all helped in one way or the other for the completion of this project. They all have provided their insights, helped me troubleshoot and have made an excellent joyful work environment.

I appreciate my research team for their time and contributions towards successful completion of this project. Kiara Smith and Alejandro Nava (Alex) spent junior and senior years of their undergraduates with me. While they learned lab skills from me, I was learning how to stay cool and enjoyable despite School's stress. Luke Schroeder joined my team in his freshman year, and now he is entering in his junior year. As I have observed him growing as a good researcher, his enthusiasm to learn more and ask critical questions has always led me think deep. Despite heavy loads of course works, Luke has helped me even during weekends and holidays. Because of his eagerness in research, he received an Undergraduate Mentor-Mentee Award to conduct an independent research and the study was well-received in Louisville Research Showcase 2020. I also had an opportunity to train Jaleyea Foster (Jay) and Noela Botaka, along with Kiara and Alex in BIOL 416. During that period, they helped me with making constructs and plant infection. Alicia Orgel, while working on her graduate project, has helped me with lots of bacterial cloning experiments.

This project would not have been possible without support from funding sources: IRIG Grant from the Office of the Vice President for Research to M.H.P. and a Research 
Grant from the College of Arts and Sciences at the University of Louisville. This work has also been partly supported by Graduate Network in Arts and Sciences (GNAS) and an Intramural grant from College of Arts and Sciences, awarded to me. I am also thankful to GNAS, the Biology Graduate Student Association (BGSA) and Fungal Conference for providing me Travel Awards to attend $2^{\text {nd }}$ International Ustilago-Smut Convergence and $30^{\text {th }}$ Fungal Genetics Conference in 2019 at the Asilomar Conference Grounds, Pacific Groove, CA. I am also grateful for the Graduate Teaching Assistantship and the Financial Aid from University of Louisville to cover my tuition and basic expenses.

I am thankful to the University of Louisville and the graduate selection committee of the Department of Biology for giving me the opportunity to pursue my Ph.D. degree in MCDB-Biology. I express my thanks to Dr. Ronald Fell, Dr. Perri Eason, Dr. Sarah Emery, Doris, Terri and all the faculty and staff of the Department of Biology for their direct or indirect help and support.

I am always grateful to my parents Suresh Pd. Khanal and Sudha A. Khanal for their unconditional love, tremendous support and constant encouragement. I am thankful to God to send me an angel in the form of my sister, Sujita Khanal. Without my sister's belief in me, I wouldn't have come this far. Heartful thanks to her for cherishing my childhood and always being there for me in joys and pains. I would also like to thank my brother-in-law, Saibyasachi (Sabby) Nath for his support and encouragement. Immense love to my nephew, Aryan Khanal Nath (my bhuntey), who always cherish me with his silly acts and gibberish talk. 
I must thank my aunt Dr. Suman Sharma and uncle Dr. Mohan Sharma, who have always encouraged me and my sister, appreciated our endeavors and are always proud of our achievements. Their motivation in all years from my high school to graduate had a profound influence on my development to reach the position where I am today. I am thankful to my roommate/ friend Abbegail (Abbey) Bell for being excellent "my firstever" roommate and making nice and friendly environment in an apartment. She has not only taught me a lot about American food and culture but also how to be organized and prompt at the same time. I would also like to thank my local guardians Jay and Linda Spading who are always there when I missed my parents back home. They are always excited to hear how my project is going on, even though they don't understand much about it.

Finally, I would like to thank all my relatives and friends who have always cherished me in my lows and helped me in one way or the other to make this dissertation successful. I remember and acknowledge all of you even though I have not listed your names here. 


\title{
ABSTRACT \\ GENES INVOLVED IN NITROGEN ASSIMILATION IN USTILAGO MAYDIS
}

\author{
Sunita Khanal
}

July 30, 2020

Ustilago maydis is a basidiomycete phytopathogen that induces tumors or galls in corn plants, hence its common name of "corn smut". The biotrophic fungus undergoes different morphological transitions throughout its life cycle. The most prominent and important transition is the dimorphic switch from budding to filamentous growth. The infectious dikaryotic filaments are produced via mating with partners of compatible mating types. The mating and subsequent infection of the host plant are the obligate parts of its sexual reproduction and life cycle. The pheromone-receptor response of compatible opposite mating partners is required for the mating. In addition, nutrient starvation, specifically nitrogen limitation is one of the environmental cues that triggers mating in this fungus.

U. maydis can utilize nitrate as a source of nitrogen from its environment, a process requiring a nitrate transporter to bring the nitrate inside the cell. This is followed by a two-step reduction of nitrate to ammonium. At first, nitrate reductase reduces nitrate into nitrite; in the second reaction, nitrite is reduced to ammonium by nitrite reductase. 
The goal of the study was to identify the nitrate transporter and nitrite reductase genes in U. maydis and characterize them based on their role in mating and virulence.

The genes um03848 and um03849 were identified bioinformatically as encoding, respectively, the nitrite reductase and nitrate transporter in U. maydis. In this study, the deletion mutants for um03848, um03849 or both genes were constructed. In addition, the CRISPR-Cas9 gene-editing technique was used to create INDEL mutations in the $U$. maydis um03849 gene. The gene deletion or editing was done in mating compatible haploid strains $1 / 2$ and $2 / 9$. For all the mutants, the phenotypes such as the growth ability, mating efficiency and pathogenesis were examined.

For the CRISPR-Cas9 edited um03849 gene, DNA sequence analysis confirmed isolates with $3 \mathrm{bp}$-deletion, $19 \mathrm{bp}$-deletion and $2 \mathrm{bp}$-substitution in the $1 / 2$ mating strain, while a 3 bp-deletion and a 66 bp-insertion were found in independent isolates of the 2/9 strain. The mating assay in these mutants showed that none of these mutations in um03849 in U. maydis affected mating with its compatible partner, as assessed by "fuzz" on charcoal media. However, the growth of mutated 1/2 strains was affected when grown in a medium with nitrate or nitrite as a source of nitrogen. With respect to host plant pathogenesis, the $1 / 2$ strain with a 2 bp substitution (resulting in a Arg-> Lys missense mutant) and frameshift mutation showed dramatically reduced infection.

In addition to growth and virulence of the fungus, other phenotypes were characterized in the mutants. Though no effect on mating was seen with deletion of either gene um03848 or um03849 alone, deletion of both genes affected the mating. The growth of all deleted mutants was impaired when grown in a medium with nitrate as a source of 
nitrogen. For the double deletion mutant, $\Delta u m 03848 \Delta u m 03849$, the growth on different stress media was also affected, depicting the interaction of the genes. Deletion of either gene alone, or both genes, led to reduced virulence. Further, when the deleted mutant strain was paired with wild type opposite mating-type partner, reduced virulence was observed, in a mating background specific manner. The background specific reduction of plant pathogenicity was correlated with differential expression of mating genes in $U$. maydis.

Therefore, our study showed that the phytopathogen $U$. maydis can assimilate nitrate as a source of nitrogen and this ability is required for full virulence on its host plant. 


\section{TABLE OF CONTENTS}

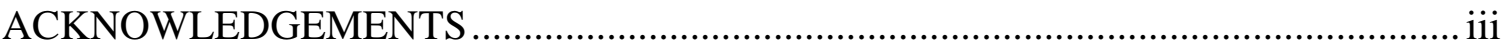

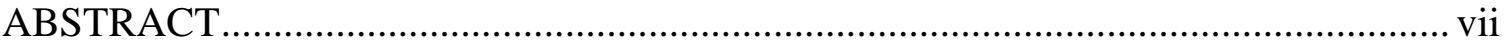

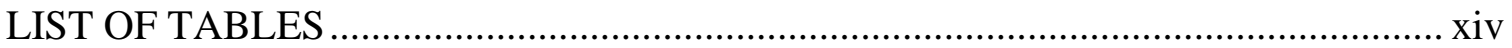

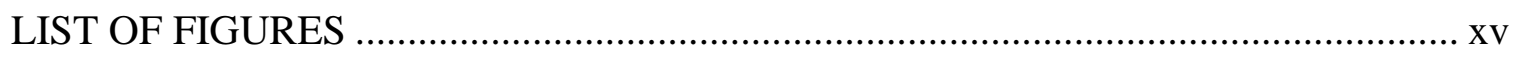

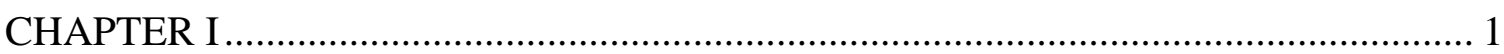

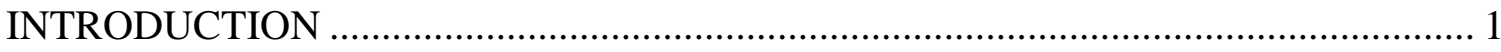

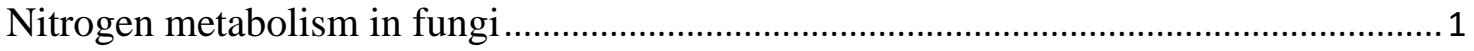

Nitrogen metabolism in Basidiomycota .................................................................

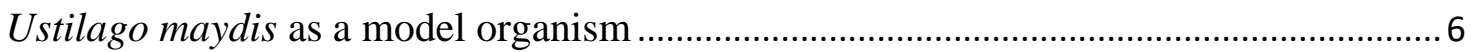

Nitrogen metabolism in Ustilago maydis .........................................................................

Lifecycle of Ustilago maydis .................................................................................

Mating genes in $U$. maydis .................................................................................10

Signaling systems for mating and pathogenesis in U. maydis .......................................12

Regulation of growth, mating process and dimorphism in $U$. maydis based on nitrogen

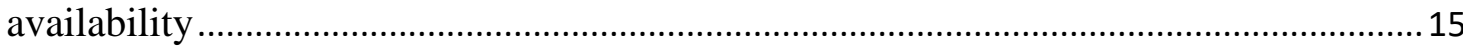

Regulation of plant pathogenicity of $U$. maydis based on nitrogen availability .............16

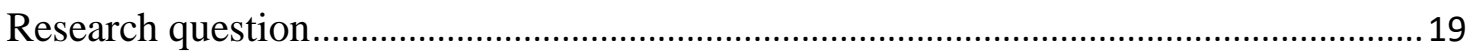

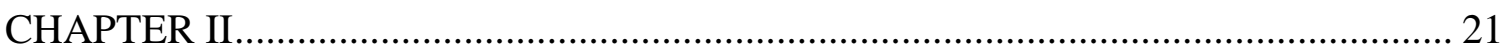

THE NITRITE REDUCTASE GENE, UM03848, IN MATING AND

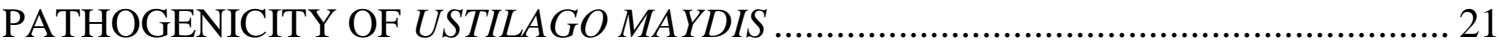

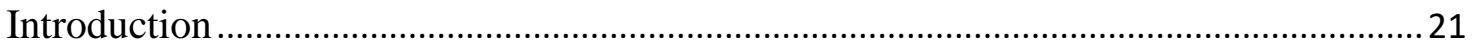

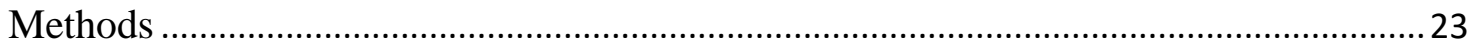

Strains and growth conditions. ........................................................................ 23 
Primer design and PCR................................................................................. 25

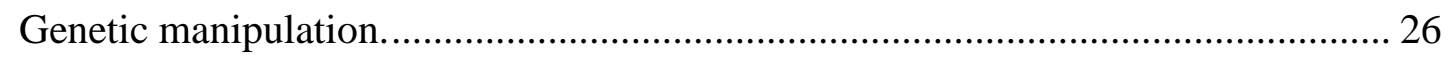

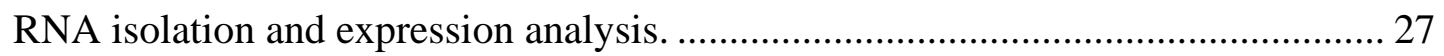

Nitrogen utilization, stress and mating assay. ……………................................... 29

Cell growth rate and infection assay.................................................................... 29

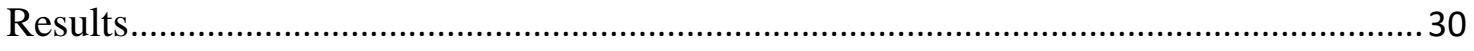

Analysis of um03848 showed high sequence similarity to known nitrite reductase from other filamentous fungi.............................................................................. 30

Induction of nitrite reductase, um03848, gene in low ammonium............................ 33

Deletion of um03848 does not affect the filamentation of $U$. maydis in low

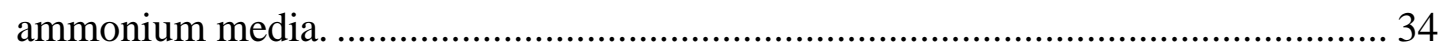

Deletion of um03848 does not affect the ability of $U$. maydis to mate with its partner.

Cell growth of um03848 mutant was not affected in rich medium, while um03848 was involved in the ability of $U$. maydis to sense and utilize nitrate as a source of

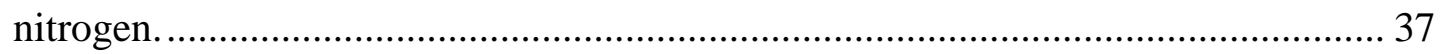

Growth of um03848 mutant in different stress media was not affected................... 43

Deletion of um03848 affects the degree of pathogenesis, in a background-specific

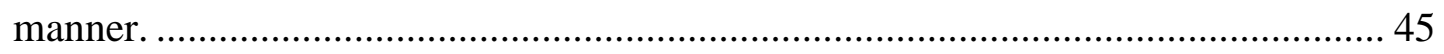

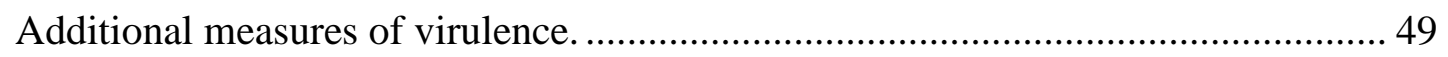

Gene expression of $u m p 2$ and mating gene targets for $\Delta u m 03848$ grown under low

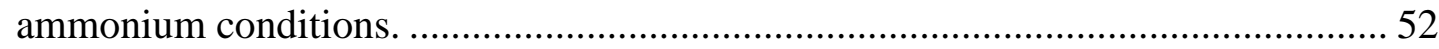

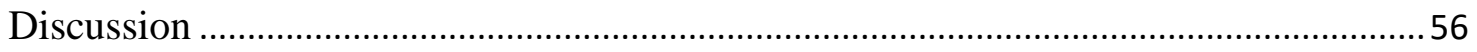

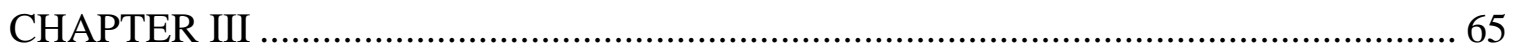

NITRATE TRANSPORTER, UM03849, GENE IN MATING AND PATHOGENICITY

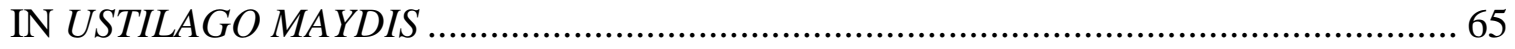

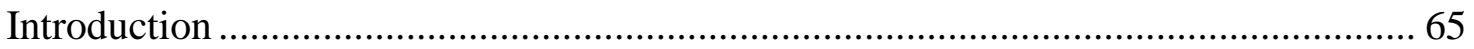

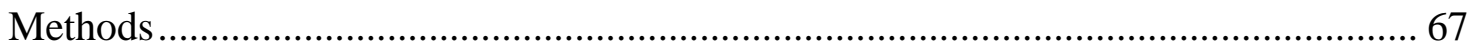

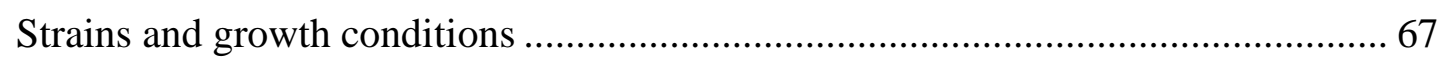

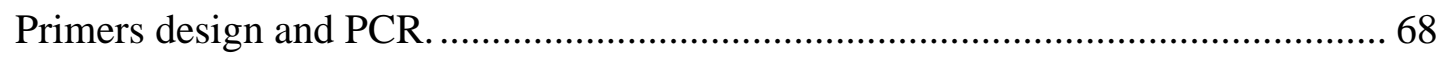

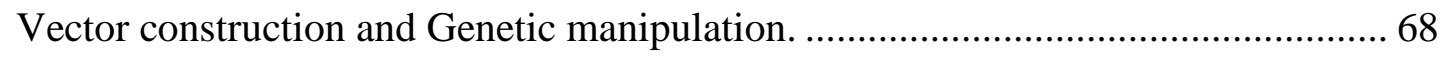

sgRNA design and construction of Cas9 vector with um03849 target...................... 69

Cell growth, nitrogen utilization, stress tests, mating and pathogenicity................... 70

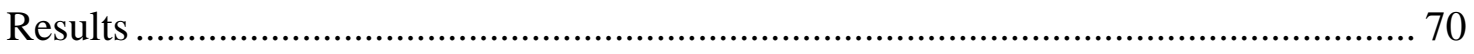


Induction of nitrate transporter, um03849, gene on low ammonium.

Deletion of um03849 (NRT) does not affect filamentation in low ammonium media.

The deletion of um03849 does not affect the growth of fungus in the medium with different nitrogen sources.

The deletion of um03849 has a slight affect on the growth of $U$. maydis in different stress media. 73

The deletion of um03849 has no obvious effect on the fungus' ability to form dikaryotic filament with its opposite partner.................................................. 75

The deletion of $u m 03849$ affects the pathogenesis to the host plant. ....................... 76

The deletion of um03849 yields differential gene expression of mating targets under low ammonium conditions. 80

Sequence analysis for the um03849-CRISPR Cas 9 mutants. 81 The um03849-CRISPR-Cas9 mutants have background specific growth effect when grown in different nitrogen sources media.

The um03849-CRISPR-Cas9 mutants does not affect the ability of $U$. maydis to mate with its opposite partner. 85

The um03849-CRISPR-Cas9 mutants affect the virulence to the plant based on type

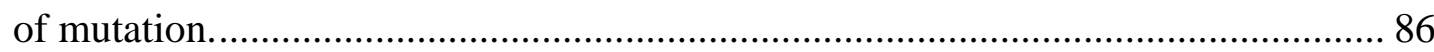

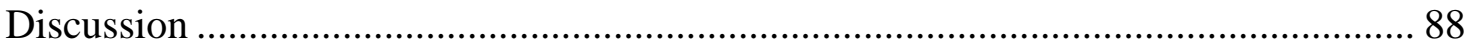

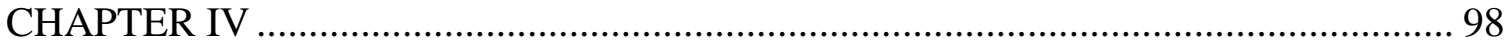

NITRATE ASSIMILATORY GENES, UM03848 AND UM03849, IN MATING AND PATHOGENICITY IN USTILAGO MAYDIS ....................................................... 98

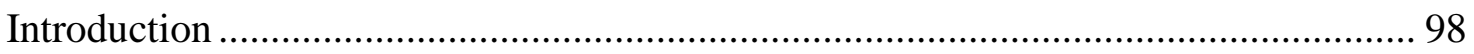

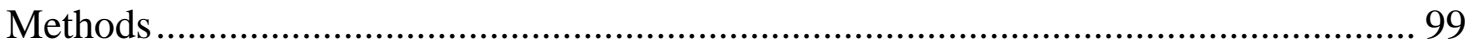

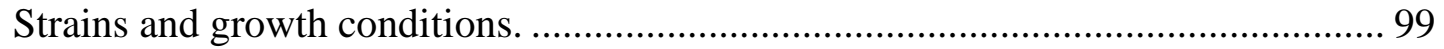

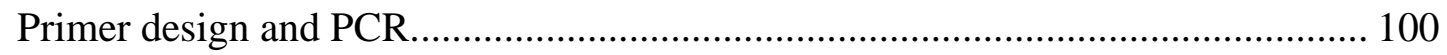

Vector construction and Genetic manipulation. ............................................... 100

Cell growth, nitrogen utilization, stress tests, mating and pathogenicity............... 101

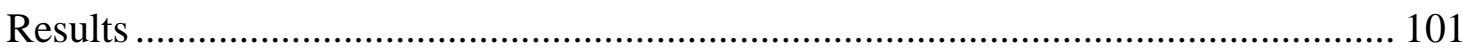

Double deletion of um03848 (NiR) and um03849 (NRT) influences filamentation in

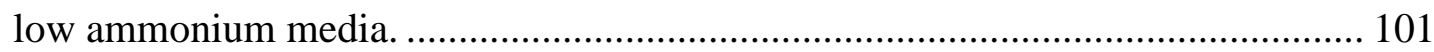

The double deletion of um03848 and um03849 affects the growth of fungus in different nitrogen media. 
The double deletion of um03848 and um03849 affects the growth of fungus in different stress media.

Double deletion of um03848 and um03849 reduces mating ability. 108

Double deletion of um03848 and um03849 reduces pathogenesis in a manner dependent on the genetic background of the mutant. 109

Discussion 112

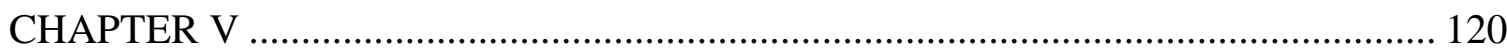

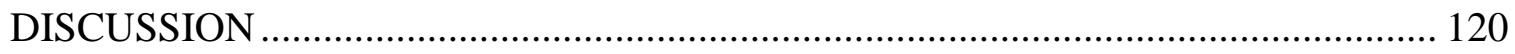

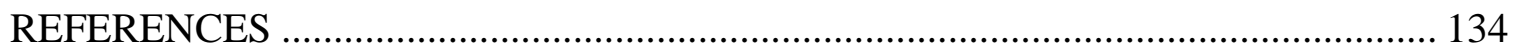

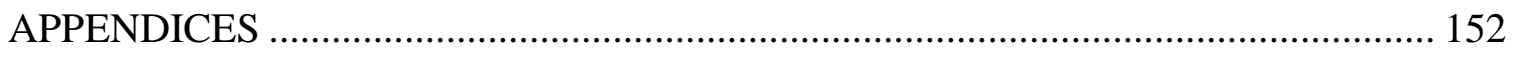

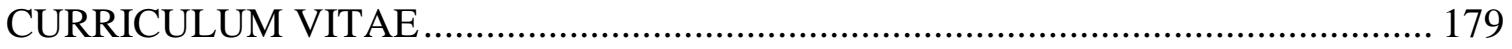




\section{LIST OF TABLES}

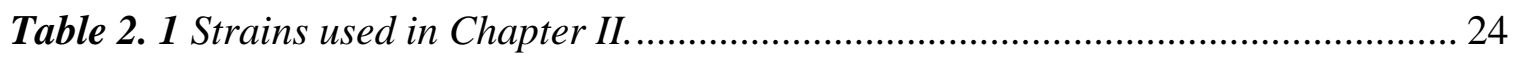

Table 2. 2 List of primers for PCR for Chapter II................................................... 25

Table 2. 3 Genes and primers used for qRT-PCR for Chapter II................................ 28

Table 2. 4 Summary of BLASTp analysis for um03848 with identified nitrite reductase. 31

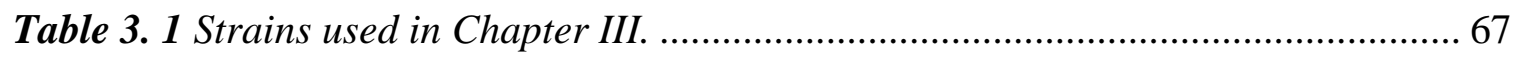

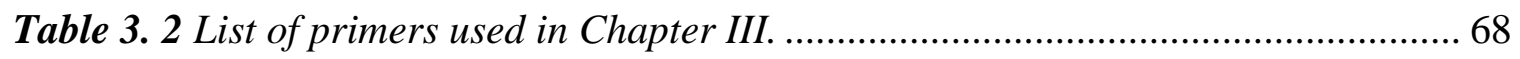

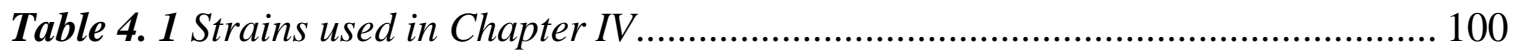

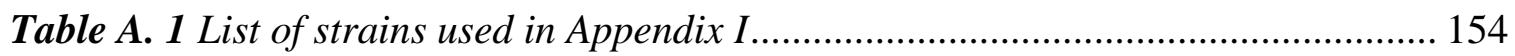

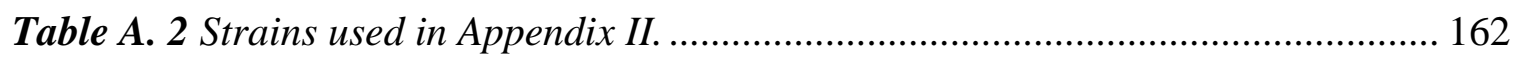

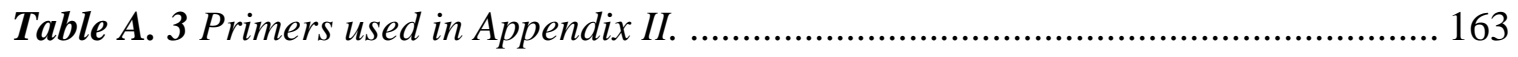

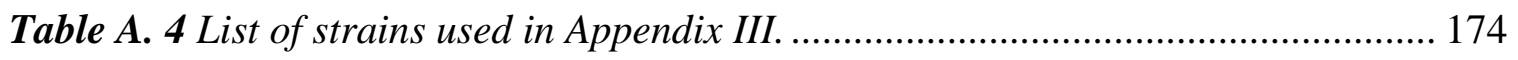




\section{LIST OF FIGURES}

Figure 1. 1 Schematic diagram of TOR kinase signaling pathway for the regulation of NCR genes expression in $S$. cerevisiae. .................................................................... 4

Figure 1. 2 Lifecycle of Ustilago maydis (Reused from Feldbrügge et al. 2004 with copyright permission). .............................................................................................. 9

Figure 1. 3 Regulation of Mating in Ustilago maydis.............................................. 12

Figure 1. 4 Co-ordination between cAMP signaling and MAPK cascade for mating and pathogenesis of Ustilago maydis. ............................................................................ 14

Figure 1. 5 Model depicting the nitrogen flow in healthy vs U. maydis infected plant. .. 19

Figure 2. 1 In silico analysis of $U$. maydis nitrite reductase, um03848, protein............ 33

Figure 2. 2 Relative expression of um03848 in wildtype grown under low ammonium

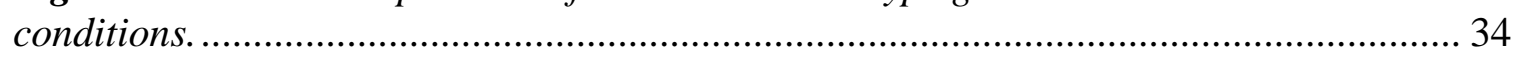

Figure 2. 3 Phenotype associated with um03848 mutants on low ammonium medium... 35

Figure 2. 4 Mating assay on PDA-charcoal plate. .................................................... 37

Figure 2. 5 Nitrogen utilization by mutants. ........................................................ 40

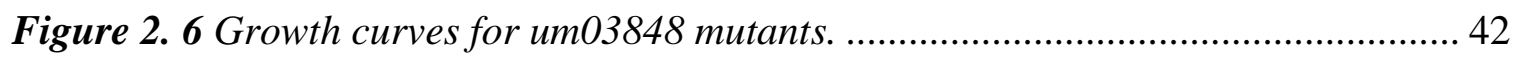

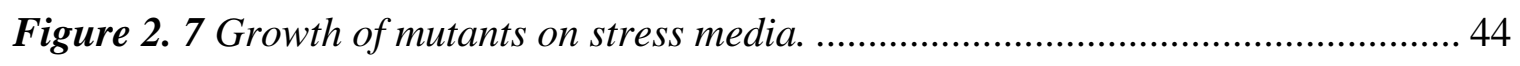

Figure 2. 8 Pathogenicity assay with indicated strains of um03848 mutants................. 45

Figure 2. 9 Plant infection using solopathogenic strain SG200 and diploid D132........ 47

Figure 2.10 Are functional copies of the um03848 gene in both partners required for

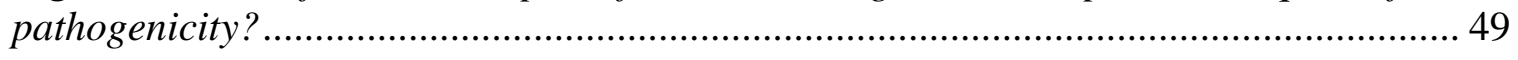

Figure 2. 11 Plant growth parameters. ................................................................ 51 
Figure 2. 12 The average log2 fold changes in different gene expression by um03848 mutants grown under low ammonium condition.

Figure 2. 13 Proposed genetic regulation of um03848 on pathogenicity of $U$. maydis... 62

Figure 3. 1 Relative expression of um03848 in wild type grown under low ammonium conditions. 70

Figure 3. 2 Phenotype observation of $\Delta$ um03849 grown on low ammonium medium.... 71

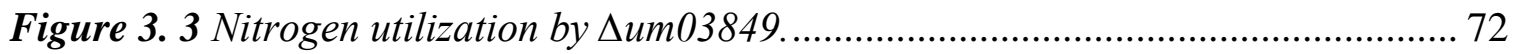

Figure 3. 4 The growth of $\Delta$ um03849 in different stress media................................... 75

Figure 3. 5 Mating assay of $\triangle$ um03849 on PDA-charcoal plate .................................. 76

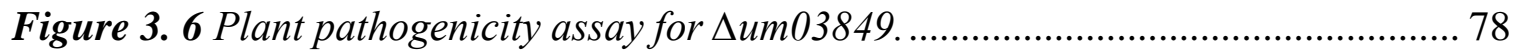

Figure 3. 7 Plant growth measurements of the plants infected by $\Delta$ um03849............... 79

Figure 3. 8 The average log2 fold differences in gene expression by $\Delta$ um03849 mutants

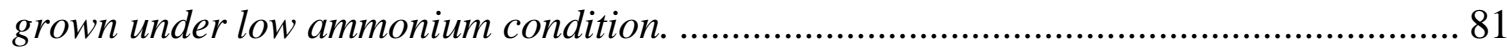

Figure 3. 9 MUSCLE alignment to evaluate INDEL mutation in um03849 gene caused by

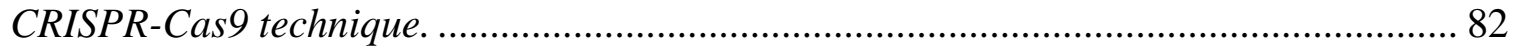

Figure 3. 10 Nitrogen Utilization by CRISPR-Cas9 mediated um03849 mutants. .......... 85

Figure 3. 11 Mating assay for CRISPR-Cas9 mediated um03849 mutants. ................... 86

Figure 3. 12 Plant pathogenicity assay for CRISPR-Cas9 mediated um03849 mutants. 87

Figure 4. 1 Phenotype observation for $\Delta$ um03848 $\Delta$ um03849 mutants grown on low

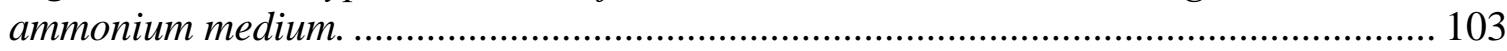

Figure 4. 2 Nitrogen utilization by double deletion mutants...................................... 105

Figure 4. 3 Growth of double deletion mutants in stress media. ............................... 107

Figure 4. 4 Mating assay of $\Delta u m 03848 \Delta$ um03849 on PDA-charcoal medium............ 108

Figure 4. 5 Pathogenicity assay for $\Delta$ um03848 $\Delta$ um03849 mutants. .......................... 110

Figure 4. 6 Plant growth measurements for the plants infected by $\Delta$ um03848 $\Delta$ um03849. 
Figure 5. 1 The average log2 fold changes in um03848 gene expression by $\Delta u m 03849$ mutants grown under low ammonium condition.

Figure 5. 2 Prediction of protein-protein interaction between nitrogen transporter/ permease in $U$. maydis.

Figure 5. 3 Proposed mode of actions for um03848 and um03849 in U. maydis.......... 133

Figure A. 1 Phenotype changes related to deletion of um03848 on FB1 and FB2 strains.

Figure A. 2 Log2 Fold changes in expression of mating targets and ump2 on wild type and mutant strains on low ammonium medium. 159

Figure A. 3 Prediction of localization of um03848 protein. 166

Figure A. 4 Agarose gel electrophoresis to determine the orientation of um03848 in transformed cells. 167

Figure A. 5 Relative expression of um03848 in transformed S. cerevisiae. 168

Figure A. 6 SDS-PAGE to separate proteins present in crude extract of transformed S. cerevisiae and $E$. coli. 169

Figure A. 7 Conserved Domain Analysis for um00037 amino acid sequence. 175

Figure A. 8 Mating and pathogenicity by $\Delta$ um00037 in U. maydis. 176

Figure A. 9 Relative expression of um03848 in mutants of U. maydis. 177

Figure A. 10 Relative expression of um03849 in mutants in U. maydis. 178

Figure A. 11 Relative expression of um03848 and um03849 in mutants in U. maydis. 178 


\section{CHAPTER I}

\section{INTRODUCTION}

\section{Nitrogen metabolism in fungi}

Nitrogen is essential for all living organisms, both prokaryotes and eukaryotes as it is an integral component of amino acids and nucleic acids. Nitrogen forms part of energy-transfer compounds such as ATP (Adenosine Triphosphate), which will allow cells to conserve and use energy released via metabolism. Thus, nitrogen is essential for growth, survival and reproduction in any organism. The nitrogen cycle converts atmospheric nitrogen into compounds that plants, animals and fungi can use ${ }^{1,2}$. Fungi can use diverse compounds as a source of nitrogen. However, fungi prefer nitrogen compounds such as ammonium and glutamine, that are easy to metabolize at lower cost of energy ${ }^{3,4}$. The coordinated regulation of distinct nitrogen metabolism of different nitrogen sources is crucial for a quick adaptation in response to the quality and concentration of available nutrients. Therefore, the cell senses the preferred (good) and non-preferred (poor) nitrogen sources in the environment. In the presence of an adequate supply of good nitrogen, the transcription of genes encoding the proteins needed to transport and degrade poor nitrogen sources are turned off. On the other hand, when the amount of a good nitrogen source becomes limiting or only poor nitrogen sources are available, the genes needed for their transport and catabolism are transcribed. The 
situation whereby metabolism of complex nitrogen sources is repressed in the presence of the favored nitrogen source is called Nitrogen Catabolite Repression (NCR) or Nitrogen metabolite repression (NMR) ${ }^{3,5,6}$.

In eukaryotic organisms, the NCR effect has been well investigated in the yeast, Saccharomyces cerevisiae, and some filamentous fungi of the Phylum Ascomycota ${ }^{7}$. The GATA factors are the major transcription factors regulating gene expression for the nitrogen catabolic activities in all fungal species. The target of the GATA family proteins is a promoter sequence containing GATA, with the C-terminus of the zinc-finger GATA factor binding to DNA ${ }^{8}$.

S. cerevisiae has four GATA factors that have been shown to mediate nitrogenresponsive gene expression. It has a pair of transcriptional activators, Gln3 and Gat1/Nil1 and a pair of transcriptional repressors Dal80 and Deh1/ Gzf3 ${ }^{9,10}$. The intracellular localization of the two GATA-family transcription activators Gln3 and Gat1/Nil1 determines the regulation of NCR genes. In nitrogen-rich medium, Gln3 and Gat 1 are retained in the cytoplasm and transcription of NCR genes is restricted to low basal levels. The sequestration of Gln3 is dependent upon Ure2, a cytoplasmic protein and Sit4, a type 2A-related phosphatase ${ }^{11,12}$. These proteins are linked to the target of rapamycin (TOR) signaling pathway. TOR is a phosphatidylinositol kinase-related protein kinase. Rapamycin is an immunosuppressive and anticancer drug that acts by inhibiting TOR. The TOR protein signaling pathway is a highly conserved pathway in most fungi that activates cell growth in response to nutrient availability and uptake ${ }^{10,13}$. TOR kinases control cytoplasmic protein synthesis and degradation through the conserved Tap42 protein (a phosphatase-associated protein of $42 \mathrm{kDa}$ ). Tap42 is an essential, conserved 
protein that independently associates with phosphatase Sit4 in response to the TOR activity ${ }^{14,15}$. TOR proteins regulate the NCR in S. cerevisiae by preventing the transcription of genes that are expressed upon nitrogen limitation. The repression of genes is promoted by binding of GATA transcription factor Gln3 to Ure2, resulting in a Gln3-Ure2 complex. In the presence of a good nitrogen source such as ammonium or glutamine, TOR keeps Sit4 inactive by promoting the association of Sit4 to Tap42. Upon nitrogen starvation, Sit4 is dissociated from its inhibitor Tap42. The activated Sit4 in turn dephosphorylates Gln3 and releases it from association with Ure2. The dephosphorylated active form of Gln3 relocates to the nucleus and binds to the DAL5 promoter (Figure 1.1), regulating the transcription of genes involved in the assimilation of alternative nitrogen sources ${ }^{13,14,16}$. Rapamycin also induces a nitrogen starvation response by inactivating TOR and increasing expression of genes involved in the uptake and use of poor nitrogen sources. When a cell is treated with rapamycin, it interacts with intracellular 12-kDa FK506-rapamycin-binding protein (FKBP12). This FKBP12rapamycin complex then acts on and inhibits the activation of TOR kinase ${ }^{17,18}$. In contrast, if a "good" nitrogen source is available again, Gln3 can exit from the nucleus and this represses the transcription of NCR genes ${ }^{11}$. However, the second GATA activator Gat1 is regulated differently than Gln3; nuclear localization of Gat1 is not strongly influenced by the TOR pathway. Gat 1 can bind to the DAL5 promoter in the absence of Gln3 but Gln3 binding to DNA is Gat1-dependent ${ }^{12}$. The GATA transcriptional repressors Dal80 and Deh1/ Gzf3 have high levels of sequence similarities in the zinc finger regions to the corresponding regions of activators Gln3 and Gat1/ Nill. Therefore, Gln3 and Gat1 must compete with Dal80 and Deh1 for binding to the GATA 
sites located in promoters of NCR-responsive genes. The outcome of this competition would influence the transcriptional activation or basal-level transcription of NCR gene expression ${ }^{19,20}$.

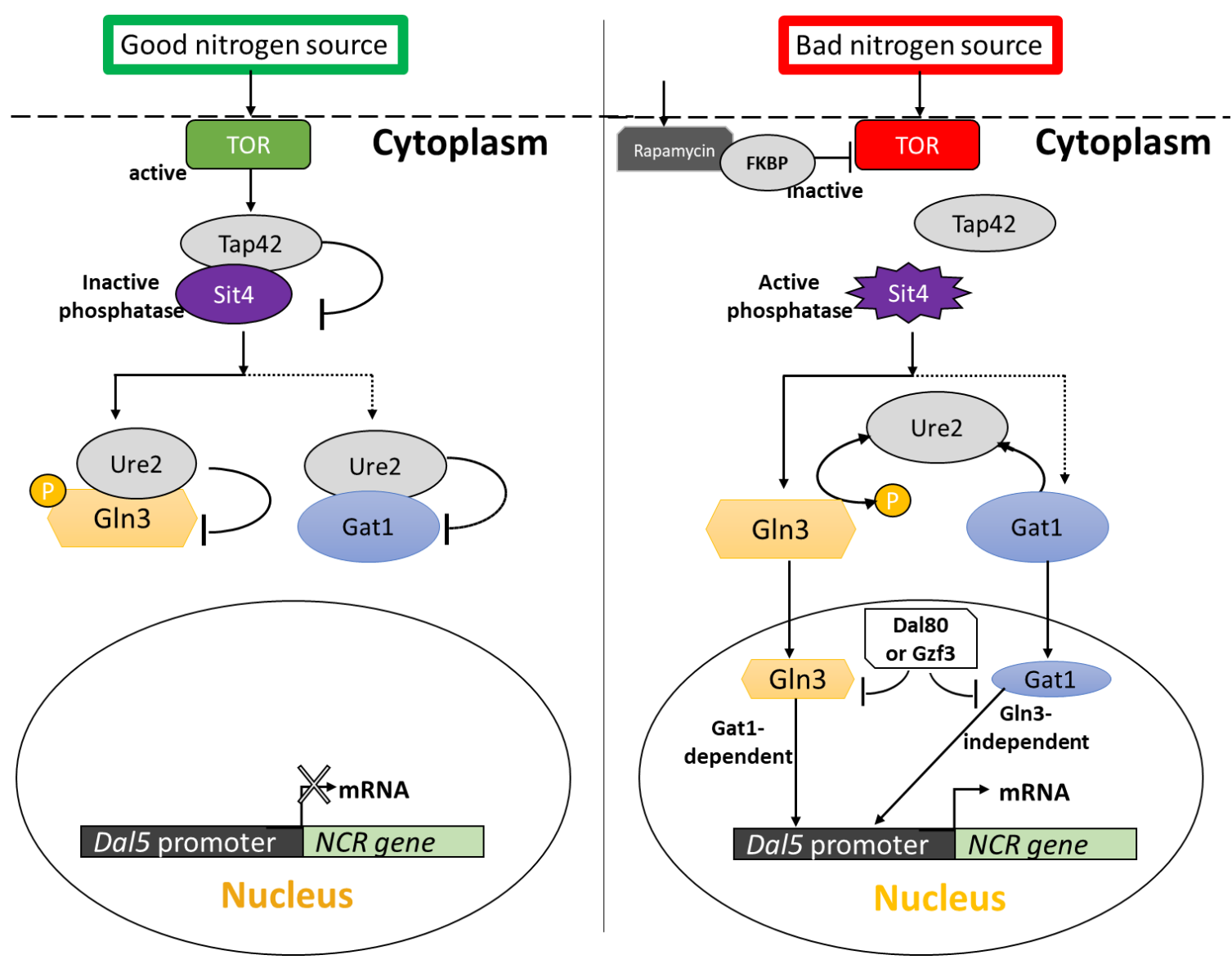

Figure 1. 1 Schematic diagram of TOR kinase signaling pathway for the regulation of $N C R$ gene expression in $S$. cerevisiae.

Under good nitrogen conditions, TOR promotes a cytoplasmic Gln3-Ure2 complex via Tap42-mediated inhibition of phosphatase Sit4, thereby preventing nuclear accumulation of Gln3 and repressing transcription of GATA-controlled genes. In presence of bad nitrogen source or rapamycin treatment, Sit4 is released from Tap42 which then dephosphorylates Gln3. Dephosphorylated Gln3 translocates into nucleus where it activates transcription of NCR genes. Dotted line represents possible regulation.

In the filamentous fungi Aspergillus nidulans and Neurospora crassa, the GATA transcription factors are AreA and Nit2, respectively, which are the central regulators of 
NCR ${ }^{3,21}$. In A. nidulans, ammonium and glutamine are utilized preferentially but, in their absence, AreA regulates a wide range of genes involved in metabolizing alternative nitrogen sources by binding to the promoters of these genes ${ }^{21,22}$. The activity of AreA is modulated via different mechanisms, including the rapid degradation of the areA transcript ${ }^{21,23}$, direct interaction with the negatively acting regulatory protein $\mathrm{NmrA}^{3,24}$, and AreA-mediated chromatin remodeling that increases histone acetylation and binding of specific transcriptional activators ${ }^{22,25-27}$. Nit2 found in N. crassa is the homolog to AreA in $A$. nidulans ${ }^{28}$. The deletion of areA or nit 2 gene leads to the inability of these filamentous fungi to utilize any nitrogen sources other than glutamine and ammonium 5,29-31. While AreA/ Nit2 proteins cause the repression of preferred nitrogen-utilizing genes, additional pathway-specific activators are required for the activation of NCR genes. For nitrate utilization, a Gal4-like transcription factor called NirA and Nit4 in $A$. nidulans and $N$. crassa respectively, induces the expression of nitrate reductase gene, nit3, to utilize nitrate as a source of nitrogen, only in the presence of AreA/Nit2 ${ }^{32}$.

The role of AreA/Nit2 orthologs has been studied in some plant pathogenic ascomycetes, where it also regulates the utilization of different nitrogen sources ${ }^{33}$. In some of the ascomycetes, such as Fusarium oxysporum ${ }^{34}$, Gibberella fujikuroi ${ }^{30}$, Colletotrichum lindemuthianum ${ }^{35}$, and Cladosporium fulvum ${ }^{36}$, mutants of the AreA/Nit2 orthologs are unable to utilize non-preferable nitrogen sources and reduce production of secondary metabolites and /or reduce pathogenicity to the host plant. 


\section{Nitrogen metabolism in Basidiomycota}

Very few reports exist on the regulatory mechanisms for nitrogen utilization in basidiomycetes. Basidiomycetes possess genes coding for GATA activators homologous to AreA/Nit2 but lack the orthologs of the GATA repressors that are known in yeast and some filamentous fungi ${ }^{4,37}$. In the human pathogen, Cryptococcus neoformans, the Gat1 gene, ortholog to AreA in A.s nidulans, regulates nitrogen catabolite repression ${ }^{38}$. In the ectomycorrhizal basidiomycete fungi, Hebeloma cylindrosporum ${ }^{39}$ and Laccaria bicolor (Maire) ${ }^{40}$, the transcription of nitrate utilization genes is repressed by the presence of ammonium in the medium but the mechanisms for that remain unknown.

\section{Ustilago maydis as a model organism}

Ustilago maydis is a biotrophic basidiomycete fungus. This means that it requires live tissue of its host plant in order to thrive and complete its otherwise pathogenic or parasitic lifecycle. This smut fungus undergoes different morphological transitions throughout its lifecycle; the most prominent and important is the dimorphic switch from a yeast-like nonpathogenic form to filamentous pathogenic form ${ }^{41}$. It is a ubiquitous pathogen of Zea mays (maize or corn) with a high degree of host specificity ${ }^{42}$. This pathogenic fungus has been long used as an important model organism for the study of morphogenesis, mating and signaling ${ }^{43}$. It is among the most genetically well-developed model plant pathogens for the basidiomycetes, used to unravel the molecular mechanisms of fungal dimorphism and pathogenicity for the rust and smut diseases ${ }^{43,44}$. U. maydis can be propagated in the laboratory in the haploid budding form and it can be

manipulated efficiently by reverse genetics ${ }^{45}$. The $U$. maydis genome is relatively small 
in comparison to other fungal plant pathogens, with fewer than 7000 predicted proteinencoding genes ${ }^{46}$. Expressed sequence tag (EST) libraries of $U$. maydis diploid teliospores and haploid cells, as well as whole-genome microarrays have provided the key resources for experiments ${ }^{47}$. In addition, $U$. maydis has been considered for biotechnological applications such as production of lipases and secondary metabolites 48,49 .

\section{Nitrogen metabolism in Ustilago maydis}

The NMR control in $U$. maydis is a global regulator, suggesting that there may be a single step mechanism for regulating expression of genes for metabolism of alternate nitrogen sources ${ }^{47}$. Ho, Cahill, and Saville 2007 identified that the um02808 and um10417 genes in U. maydis share functional homology to Nit4 and Nit2/AreA-like regulator, respectively. These genes are believed to control the alternate nitrogen

utilization in $U$. maydis ${ }^{47}$. The gene um10417 in $U$. maydis has homology to Gat1p from S. cerevisiae, as both contain the conserved Zn finger domain. Um10417 is a positive regulator of the unfavorable nitrogen source utilization pathway. It was shown that um10417 deletion in haploid sporidial strains were not able to grow on most of nitrogen source media other than ammonium and glutamine. Similarly, um10417 is also required for the efficient initiation of dikaryotic filamentation growth and virulence on its host maize plant ${ }^{6}$. A BLAST in U. maydis genome performed by Romero-Aguilar et al. 2020 found that this fungus has one TOR kinase. Treatment with rapamycin resulted in large morphological changes such as alternations in cell size, the production of septa, and 
different distribution of vacuoles. However, rapamycin has no effect on U. maydis vegetative growth ${ }^{50}$.

In addition, $U$. maydis nitrate reductase activity is induced by nitrate and repressed by ammonia ${ }^{51}$, suggesting a nitrogen catabolite repression (NCR) regulatory system in this phytopathogen. For utilization of nitrate, an organism needs an active transporter for nitrate, plus nitrate reductase and nitrite reductase enzymes. Nitrate is transported into the cells by nitrate transporter and it is reduced to ammonium in a twostep reduction. At first, nitrate is reduced into nitrite which is catalyzed by an enzyme, nitrate reductase (NR), and the second step is the reduction of nitrite to ammonium by

nitrite reductase (NiR) ${ }^{52-54}$. In $U$. maydis, genomic analysis has identified the um03849 (or $U M A G \_11105$ ) gene encoding nitrate transporter and the um03848 (or $U M A G_{-}$11104)gene encoding nitrite reductase ${ }^{55}$. A gene encoding nitrate reductase, um03847, was previously identified ${ }^{56}$. As in other fungi, these three genes form a cluster in the U. maydis genome and oversee the reductive assimilation of nitrate and nitrite to $\operatorname{ammonium}^{55}$.

\section{Lifecycle of Ustilago maydis}

Ustilago maydis must establish a biotrophic relationship with its host corn plant to complete its lifecycle. If it is not infecting plants, it exists as a haploid yeast-like form. The non-pathogenic saprophytic haploid sporidia of $U$. maydis multiply asexually by budding (Figure 1.2). When the compatible haploid partners of opposite mating type recognize each other, they undergo conjugation resulting in a dikaryon that can infect the host corn plant by forming an appressorium. Local growth inside the plant tissue leads to 
tumor formation where nuclear fusion and hyphae fragmentation results in numerous diploid teliospores. Bursting of tumors releases teliospores into the environment, which remain relatively dormant until favorable conditions. When diploid teliospores germinate, they undergo meiosis and again produce haploid sporidia ${ }^{45}$.

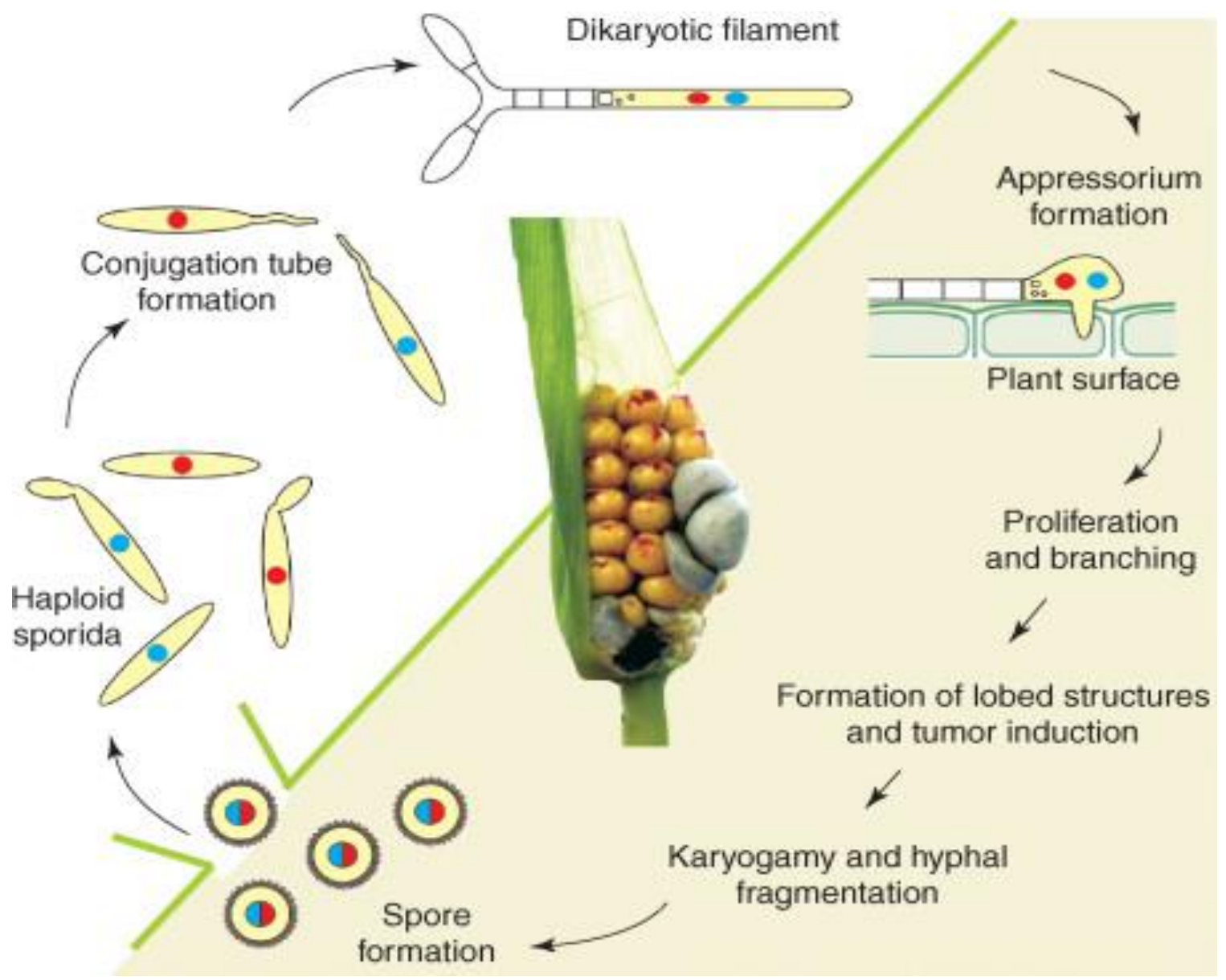

Figure 1. 2 Lifecycle of Ustilago maydis (Reused from Feldbrügge et al. 2004 with copyright permission).

The blue and red circle inside filament represent opposite mating type nucleus. The yellow shades of the figure represent the lifecycle inside host plant. 


\section{Mating genes in $U$. maydis}

U. maydis uses a tetrapolar mating system that consists of two MAT (matingtype) genetic loci with at least two alleles at each locus. In $U$. maydis, the two mating loci are $a$ and $b$, where the $a$ locus governs cell fusion between haploid partners and the $b$ locus controls the sexual development after fusion, and pathogenicity. Therefore, the mating compatibility of $U$. maydis is regulated by the gene products of $a$ and $b$ loci at the pre- and post-fusion levels, respectively ${ }^{41,43,57,58}$.

The allele at the $a$ locus is identified as either $a 1$ or $a 2$, depending on the mating type strains, i.e., the $a 1$ allele is specific to a1 strains. Molecular analysis has revealed that the $a 1$ allele is smaller, at $4.5 \mathrm{~kb}$, while the $a 2$ allele is larger, with $8 \mathrm{~kb}^{59,60}$. The biallelic $a$ locus (al/a2) encodes an intercellular recognition system consisting of lipopeptide pheromone precursors ( $\mathrm{mfal} / \mathrm{mfa} 2)$ and their receptors ( $\mathrm{pral} / \mathrm{pra2})^{59}$. When compatible mating partners (a1b1/a2b2) come into proximity, usually on the plant surface, the pheromone produced by one mating type (a1b1) is recognized by the corresponding receptor of the opposite mating type (a2b2). This begins a cascade of signaling events that alters gene expression in the $a$ and $b$ loci ${ }^{43}$.

The multiallelic $b$ locus encodes the two divergently transcribed genes, $b E$ ( $b$ -

East) and $b W$ ( $b$-West), encoding 410- and 626-aa proteins bE and bW, respectively ${ }^{43,61}$. These two homeodomain proteins are mostly unique in the $\mathrm{N}$ terminal region ${ }^{61}$. The $\mathrm{N}$ terminal region mediates the heterodimerization of these proteins, generating active transcription factors that leads to sexual development. This heterodimerization, mediated 
by the $\mathrm{N}$ terminus, is an essential component of self- versus non-self- recognition in the mating partners ${ }^{62}$.

When two compatible haploid sporidia come closer and recognize each other, the lipopeptide pheromone $(\mathrm{mfa} 1 / 2)$ secreted by one mating partner will bind to the receptor (pra1/2) of the opposite partner (Figure 1.3) by forming conjugation tubes towards each other. The stable dikaryon is formed only if they contain different alleles of the multiallelic $b$ locus which is then able to develop an appressorium and infect corn plants ${ }^{58}$. The successful mating then activates the series of signal cascades leading to the formation and activation of transcription factor, Prf1. Prf1 regulates the pheromoneinduced expression of the $a$ and $b$ mating type genes ${ }^{63}$. Upon fusion of an $a 1 b 1$ haploid cell to an $a 2 b 2$ haploid cell, the bE protein of a mating type will physically interact with a $\mathrm{bW}$ protein of the opposite mating type to form the functional heterodimer, for example bE1 dimerization with bW2, forming bE1-bW2 heterodimer. The heterodimer acts as a transcription factor for the genes necessary to establish the infectious dikaryon and establishment of pathogenicity to the host plant ${ }^{43,59,64,65}$. Therefore, the successful transition from budding to dikaryon critically depends on an active bE/bW heterodimer. In addition, the active $\mathrm{bE} / \mathrm{bW}$ heterodimer differentially regulates the expression of genes involved in cellular processes such as cell wall remodeling, lipid metabolism, cell cycle control, mitosis and DNA replication. If the promoter lacks putative $\mathrm{bE} / \mathrm{bW}$ binding sites then $\mathrm{bE} / \mathrm{bW}$ heterodimers induce other transcriptional regulators such as Rbf1 to regulate gene expression ${ }^{66}$. 


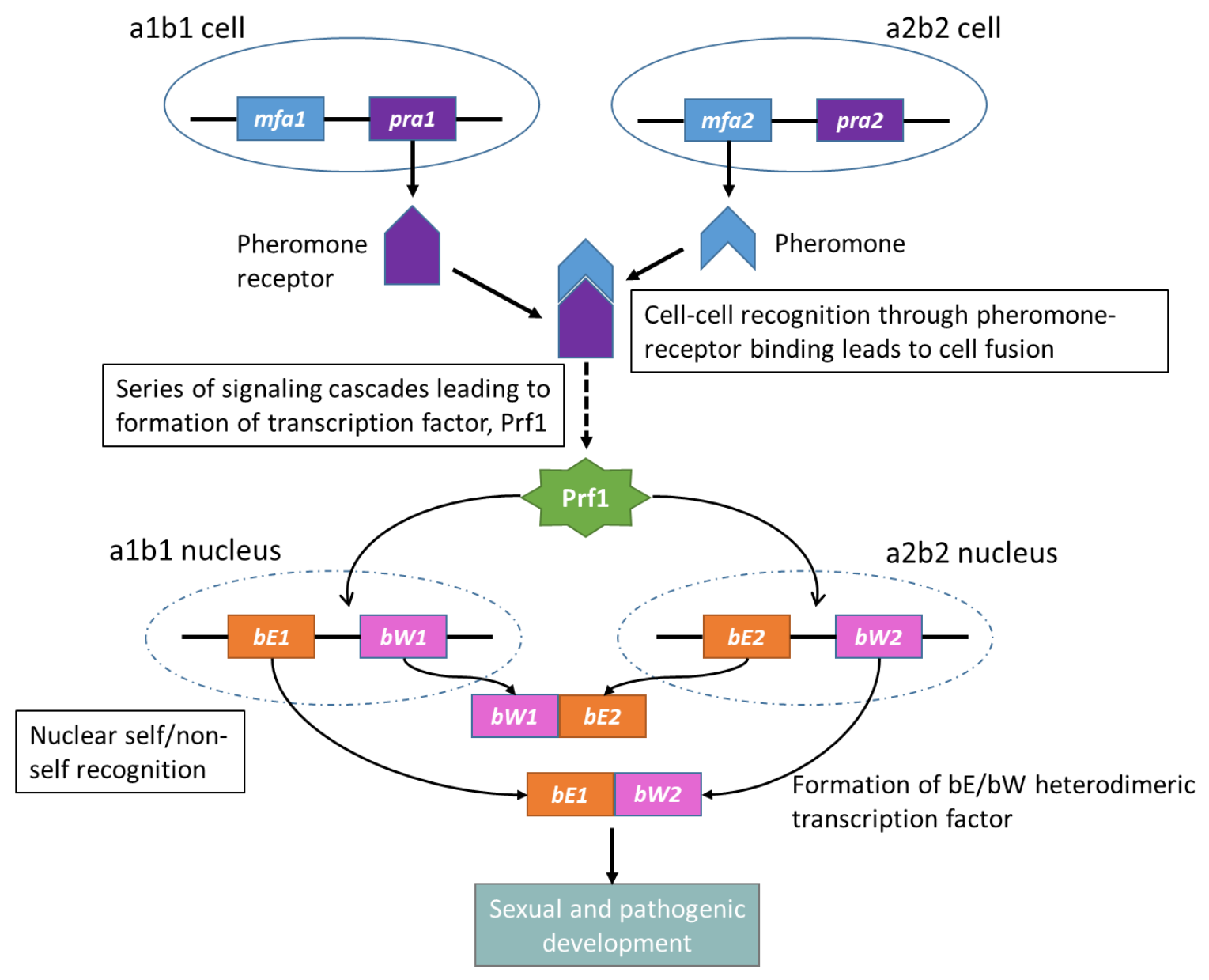

Figure 1. 3 Regulation of Mating in Ustilago maydis.

\section{Signaling systems for mating and pathogenesis in U. maydis}

The signaling systems that control dimorphism, mating and pathogenesis are conserved among related fungi ${ }^{67}$. There is a crosstalk between the cAMP (cyclic AMP)dependent protein kinase A (PKA) pathway and the mitogen-activated protein kinase (MAPK) signaling for the mating and virulence in $U$. maydis ${ }^{68-70}$. The phosphorylation of Prf1 is used to integrate PKA and MAPK signaling pathways. The PKA mediated Prf1 phosphorylation sites are essential for induced expression of $a$ and $b$ mating type genes, while MAPK mediated phosphorylation is used for pheromone-induced expression of $b$ 
gene but not required for the expression of $a$ gene ${ }^{68}$. The pheromone response elements (PREs) are present in the regulatory regions of $a$ and $b$ mating type genes ${ }^{60}$, which is recognized and bound by Prf1 to control the expression of these genes.

In $U$. maydis, lipoprotein pheromone (mfa1/2) binding to its 7- transmembrane Gprotein-coupled receptor (GPCR- pra1/2) or environmental cues/nutrient starvation activate the downstream signaling cascades that lead to induction of a large number of genes (Figure 1.4). The pheromone-receptor interaction or environmental signals activate the dissociation of heterotrimeric G-protein into $G \alpha$ and $G \beta \gamma$ subunits. The liberated G $\beta \gamma$ subunit interacts with the other proteins which then activate the MAP kinase signaling pathway ${ }^{71,72}$. In U. maydis, MAPK cascades consists of MAPKKK Kpp4 or Ubc4, MAPKK Fuz7 or Ubc5, and MAPK Kpp2 or Ubc3, where each kinase is activated by phosphorylation by previous kinases upstream in the pathway. Activated MAPK subsequently phosphorylates and activates the transcription factor, Prf1 ${ }^{68,73-75}$ which, in turn, activates $a$ and $b$ genes for mating, filamentation and virulence response. 


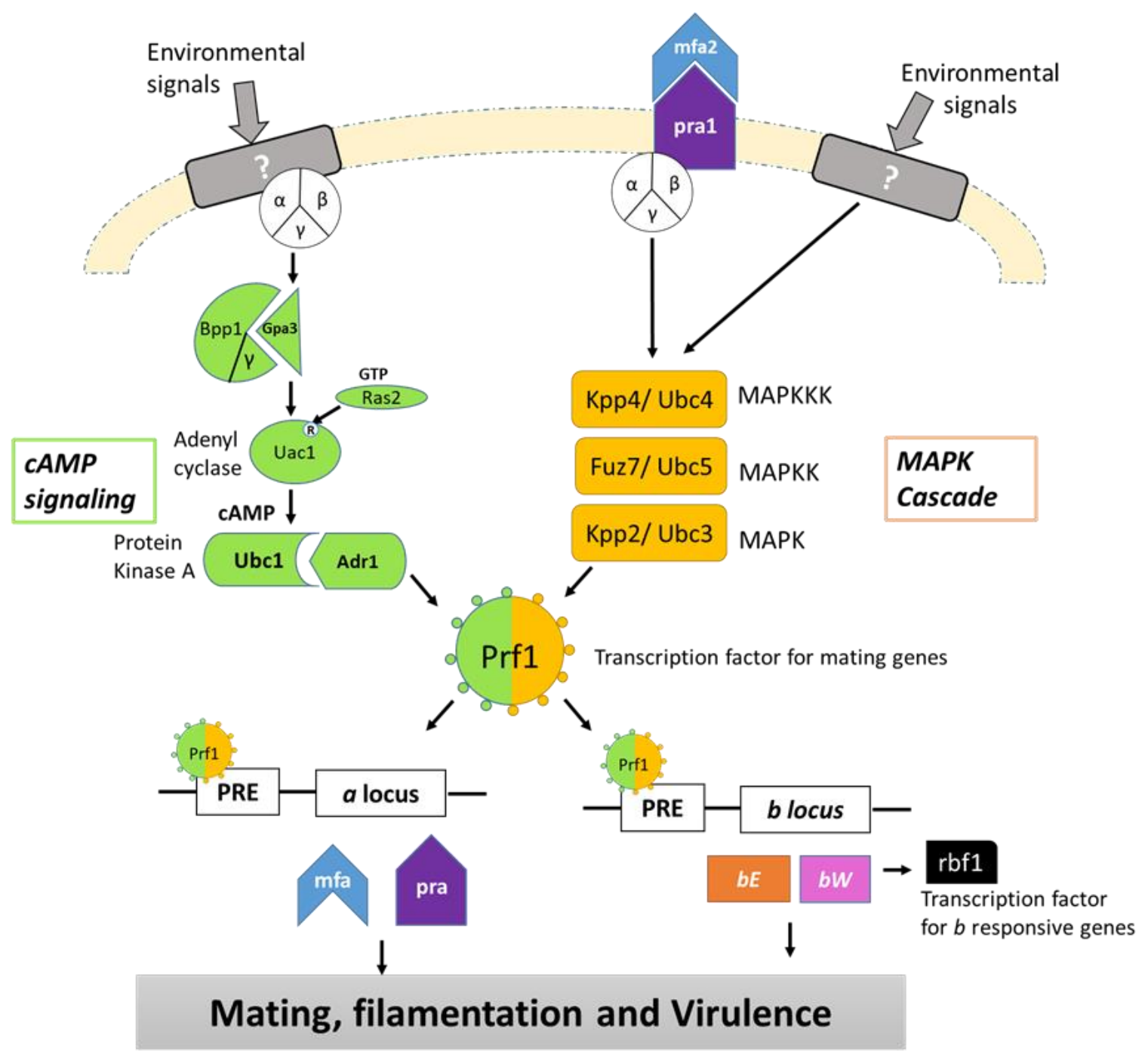

Figure 1. 4 Co-ordination between cAMP signaling and MAPK cascade for mating and pathogenesis of Ustilago maydis.

The green color represents the components of cAMP pathway and orange represents the components of MAPK pathway. Either of these pathway is able to phosphorylates and activates Prf1 while both pathways is required for regulation of mating and virulence of fungus.

In addition to the MAPK pathway, the cAMP-PKA signaling pathway also regulates mating and virulence in $U$. maydis, mostly in response to environmental signals (Figure 1.4). This pathway consists of the $\alpha$ subunit of heterotrimeric G protein, Gpa3; the adenylate cyclase, Uac1; and the dimeric protein units, Ubc1-Adr1. Ubc1 is a 
negative regulator of Adr1, which is the catalytic subunit of PKA in U. maydis. When the cell receives environmental signals (whose receptors are currently unknown), it dissociates heterotrimeric G-protein. One of the four subunits of $\alpha$ unit of G protein, Gpa3, activates the adenylate cyclase, Uac1, which leads to increased levels of cAMP. High levels of cellular cAMP cause the dissociation of the dimeric regulatory protein units (Ubc1) from the catalytic subunit Adr1. The dissociated Adr1 then phosphorylates transcription factor Prf1 which upregulates the expression mating genes ${ }^{68,76-78}$.

\section{Regulation of growth, mating process and dimorphism in U. maydis based on nitrogen availability}

Environmental signals and nutritional availability can affect cellular differentiation, and have specific roles in mating and virulence in many fungi ${ }^{79}$. For example, the diploid cells of yeast $S$. cerevisiae undergo a dimorphic transition from the yeast form to pseudo-hyphal growth in response to the limitation of nitrogen in the media; whereas in the case of haploid cells, nitrogen limitation promotes the invasive growth on solid media ${ }^{67,80}$. For Microbotryum violaceum, a phytopathogen of the flowering species of the Caryophyllaceae family, mating only occurs under starvation for nutrients, in particular, nitrogen ${ }^{79}$.

Ustilago maydis grows haploid filaments or aerial hyphae on nitrogen limited ${ }^{81}$ or on low ammonium media ${ }^{79}$. Such transitions in growth form might help the cells forage for the nutrients under limited conditions. The phenotypic changes in response to low ammonium in $U$. maydis require only the high affinity ammonium transceptor, Ump2. Deletion of ump1, encoding the low affinity ammonium transporter, did not yield 
any distinguishing growth difference from wild type when grown in low ammonium medium. However, the deletion of $u m p 2{ }^{79}$ or $b$ genes ${ }^{82}$ yielded haploid strains unable to produce filaments under similar growth conditions. Therefore, ump2 detects nitrogen availability in medium in a $b$-dependent manner and functions in filamentation ${ }^{82}$.

Furthermore, the dimorphic transition in U. maydis and its pathogenicity to the host plant are distinct in response to the environmental cues such as acid $\mathrm{pH}$ or low nitrogen availability ${ }^{69}$. In the laboratory, the formation of dikaryotic filaments by mating between opposite mating-type haploid cells is induced in nitrogen starvation conditions on solid charcoal medium ${ }^{41,79}$. In nature, mating and formation of the infectious dikaryon in $U$. maydis occurs on the aerial parts of the plant ${ }^{83}$. This indicates that the fungus gains access to the resources by invading the host tissues, as there is an established connection between the nutrient limitation and induction of mating.

\section{Regulation of plant pathogenicity of $U$. maydis based on nitrogen availability}

As already indicated, $U$. maydis is a biotrophic phytopathogen ${ }^{41,66}$ which means that it doesn't kill its host but derives nutrition and completes its lifecycle in living host plant tissue ${ }^{84}$. The establishment of a biotrophic relationship with the host corn plant is necessary for $U$. maydis to complete its lifecycle. Only the dikaryotic filamentous form can infect the plant. The dikaryotic filaments show tip-directed growth by accumulating cytoplasm into the tip cell compartment and thus tips swell to form the poorly differentiated structure known as the appressorium that directs the penetration of the filament into plant tissue ${ }^{66}$. The filamentous dikaryon proliferates in the plant tissues, growing mostly intracellularly but without much damage to the host cells. As it 
proliferates, it induces tumor formation in the aerial parts of plant. Because it is difficult to propagate filamentous dikaryon in laboratory culture media but it grows well in the plant tissues, it can be hypothesized that plant signals promote its growth and/or maintenance in the dikaryotic form ${ }^{85}$. The infection with $U$. maydis causes hypertrophy (increase in cell size) and hyperplasia (increase in cell numbers) of the plant cells of the infected region. In addition to this, increased levels of plant growth hormones such as auxins or cytokinin in the infected tissue leads to the growth of tumors ${ }^{43,86}$. The tumors provide the environment where hyphae differentiate, karyogamy occurs and production of a specialized cell wall results in diploid teliospores. Upon bursting out of the tumor, teliospores are dispersed into the environment. Teliospores can lie dormant in the soil for years. Teliospores are not capable of vegetative growth. They germinate under appropriate conditions, undergoing meiosis to produce haploid progeny that then reproduce by budding ${ }^{85}$. There is clear interaction between pathogen and host for the filamentous growth, host tissue invasion, eliciting tumor formation and fungal differentiation to teliospores and meiotic production of haploid cells. There has been intense study on deciphering these signals and identifying the genes involved in response to these signals ${ }^{45,69,85}$. However, not much is known regarding the role of nitrogen in plant-pathogen interactions for the infection process in $U$. maydis.

It has been reported that nitrogen starvation in a plant increases its susceptibility to pathogen infection, most likely caused by reduced general plant fitness ${ }^{87}$. Some studies also suggest that plants mobilize nitrogen to the infected location as a defense strategy to abiotic and biotic stresses ${ }^{88-90}$. Use of nitrogen fertilization in host plants has shown both of these two conflicting effects, as it enhances plant defense as well as 
increases the nitrogen compounds available for the pathogens in host plants. Mostly biotrophic and hemi-biotrophic pathogens benefit from increased metabolite pools in the host tissues, but not necrotrophic fungi. In addition, pathogen attack is also expected to modify plant nitrogen content in the site of infection as well as activate important metabolic changes in the whole host plant similar to those enhanced during senescence process ${ }^{87-89}$. In addition, abiotic stress and pathogen infection enhance the expression of several plant genes involved in nutrient recycling, nitrogen remobilization, proteolysis and transporters for sugars, amino acids, and sulfur ${ }^{91-93}$.

Within 12 hpi (hours post-infection) of $U$. maydis infection, there is upregulation of defense genes in the maize plant, whereas by $24 \mathrm{hpi}$, these genes are highly downregulated. From 24 hpi onwards, the genes for the metabolism of amino acids are highly upregulated, along with the genes for cell wall synthesis and lipid metabolism ${ }^{94}$. In $U$. maydis infected plants, there is significant increase of total amino acids during tumor development and it progressively increases in infected plants ${ }^{83}$. Horst et al. 2010 demonstrated that for plants with $U$. maydis-induced tumors, primary nitrogen assimilation from soil is reduced, whereas organic nitrogen from other systemic leaves preferentially accumulates in tumors compared to the uninfected plants (Figure 1.5). The rerouting of nitrogen from systemic leaves into tumors could possibly be to overcome the nutrient limitation in infected leaves. Therefore, the tumor-induced plants have higher phloem-mobility and lower xylem-mobility towards the tumor. Furthermore, it was also observed that photosynthesis is elevated in lower systemic leaves of infected plants which exhibit a delay in senescence ${ }^{95}$. This indicates that the $U$. maydis sequesters and uses 
most of the organic nitrogen in the plant for the induction and development of tumors, resulting in the elevated demand for nitrogen.

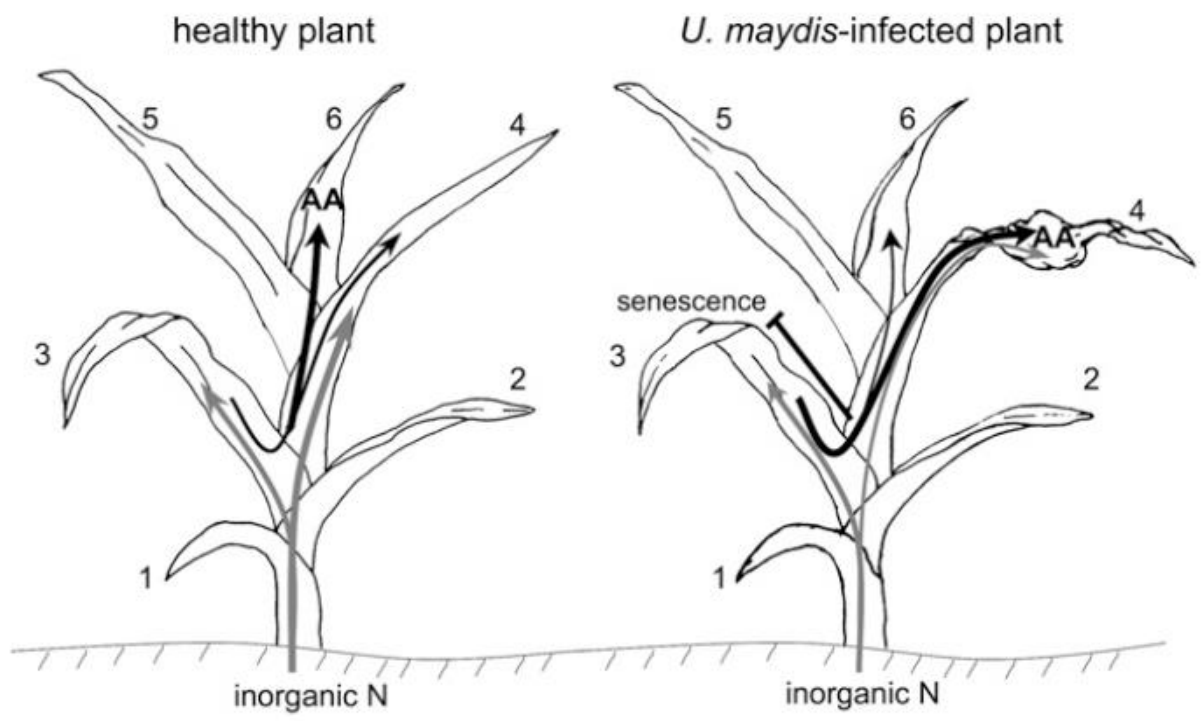

Figure 1. 5 Model depicting the nitrogen flow in healthy vs U. maydis infected plant. (Reprinted from ${ }^{95}$ with copyright permission from the American Society of Plant Biologists). The gray arrow shows the flow of inorganic nitrogen and black arrows for amino acids, AA, in healthy plant on left and $U$. maydis-infected plant on right. The thickness of the arrows indicate the flow rate ${ }^{95}$.

\section{Research question}

The regulation of nitrogen metabolism, nitrogen uptake and the effects of different nitrogen sources in dikaryotic fungi are of great interest as it has been established that mating, filamentation and induction of pathogenesis in these fungi is dependent on nitrogen limitation ${ }^{55}$. The maize pathogenic fungus $U$. maydis has been seen to grow in low or no nitrogen media. For low ammonium, strains grow at rates similar to those seen in nutrient rich media ${ }^{96,97}$. In addition to ammonium, $U$. maydis can utilize nitrate as a nitrogen source as well ${ }^{98}$. The study in our laboratory also supports the finding that $U$. maydis can grow without nitrogen being provided in the medium at all ${ }^{99}$. 
The double mutant lacking both ammonium transporters, ump1 ump2, in $U$. maydis is also able to grow on low ammonium medium ${ }^{82}$. This suggested that $U$. maydis might adopt some other pathways or might have some genes which help cells to assimilate inorganic nitrogen and assist the growth in limited nitrogen media. The present study was conducted to characterize the genes that are involved in the assimilation of less-preferred inorganic nitrogen, specifically nitrate, in $U$. maydis. The deletion and overexpression mutants of the genes involved in nitrate transport, um03849, and nitrite reductase, um03848 and um00037 (having a domain for nitrite reductase), were constructed to explore the role of these genes in the growth of the fungus under nitrogen starvation conditions. The subsequent chapters investigate the role of each of these genes in mating and plant infection of the fungus. Chapter IV focuses on the combined effect of um03849 and um03848 in the cell growth, filamentation, mating and pathogenicity of $U$. maydis. 


\section{CHAPTER II}

\section{THE NITRITE REDUCTASE GENE, UM03848, IN MATING AND PATHOGENICITY OF USTILAGO MAYDIS}

\section{Introduction}

Nitrogen limitation in Ustilago maydis is linked to mating, filamentation and induction of pathogenesis ${ }^{79,81}$. Therefore, the regulation of nitrogen metabolism, nitrogen uptake and the effects of different nitrogen sources are of interest. This study attempts to understand nitrogen assimilation in $U$. maydis, by focusing on the nitrate metabolism pathway.

The majority of fungi are able to assimilate nitrate or nitrite, which are the most abundant nitrogen ions present in soils ${ }^{100}$. Once nitrate is transported into the cell, nitrate is reduced to ammonium by the consecutive action of nitrate and nitrite reductases. Since this pathway involves a substantial investment in metabolic energy, it is tightly regulated 53,93 . In general, de novo synthesis of nitrate and nitrite reductases requires both low intracellular concentrations of preferred nitrogen sources (nitrogen-starvation) and simultaneous presence of nitrate or nitrite, which act as transcriptional inducers ${ }^{3}$. In addition, the nitrate reductase gene product appears to autoregulate its own gene expression and regulate that of the nitrite reductase ${ }^{101}$. 
U. maydis can utilize nitrate as a nitrogen source as demonstrated by its ability to grow on minimal medium containing only potassium nitrate ${ }^{102}$. Genomic analysis has identified potential genes encoding a nitrate permease/ transporter $\left(U M A G \_11105\right.$ or um03849), nitrate reductase (narl; um03847) and nitrite reductase (UMAG_11104 or um03848). These three genes form a cluster in U. maydis, as they do in other fungi ${ }^{3,56}$. The regulation of these genes provides the means for the reductive assimilation of nitrate to ammonium. Chapter II of this study is to understand the role of the nitrite reductase gene in different phenotypes of $U$. maydis.

Nitrite reductase catalyzes the reduction of nitrite to ammonium. The nitrite reductases (NiR; EC 1.7.1.4) of higher plants use ferredoxin $(\mathrm{Fd})$ as electron donor and they are found in the chloroplast, although encoded by a nuclear gene ${ }^{103}$. However, in bacteria and fungi, NiR (E.C. 1.6.6.4) uses NAD(P)H as an electron donor ${ }^{104,105}$. Both forms of NiR contain siroheme-Fe and iron-sulphur (Fe-S) centers, while fungal and bacterial NiR also contain FAD and Fe-S centers not associated with siroheme ${ }^{105,106}$. Therefore, fungal nitrite reductase catalyzes $[\mathrm{NAD}(\mathrm{P}) \mathrm{H}]$-dependent 6-electron reduction of nitrite to ammonia as shown by the reaction,

$$
\mathrm{NO}_{2}^{-}+3 \mathrm{NAD}(\mathrm{P}) \mathrm{H}+3 \mathrm{H}^{+} \rightarrow \mathrm{NH}_{4} \mathrm{OH}+3 \mathrm{NAD}(\mathrm{P})^{+}+\mathrm{H}_{2} \mathrm{O}^{104,107}
$$

In Ustilago maydis, um03848 (or UMAG_11104) has been identified as the potential gene encoding nitrite reductase ${ }^{55}$. The nitrite reductase gene, um03848, encodes a protein of 602 amino acids that contains regions corresponding to conserved assimilatory nitrite reductase family, siroheme-binding site and Rieske domain analyzed from Conserved Domain Analysis at NCBI ${ }^{46,108,109}$ (Figure 2.1 B). The bacterial NirD has a single Rieske domain while fungal proteins have a C-terminal Rieske domain in 
addition to several other domains. The Rieske domain is an iron-sulphur [2Fe-2S] cluster binding domain involved in electron transfer ${ }^{109}$.

In this chapter, we studied the role of the um03848 (NiR in U. maydis) gene in mating and virulence of the fungus. We constructed a $u m 03848$ deletion mutant and showed that the mutant not only is severely impaired for growth in the medium with nitrate as the source of nitrogen, but also affects the virulence of infection on the host plant. We further tested the degree to which a $\Delta u m 03848$ mutant was able to infect plants when mated with a wild type partner strain. We found that the deletion of $u m 03848$ in the $1 / 2$ background can significantly reduce virulence while deletion in the $2 / 9$ genetic background did not have a significant effect. In order to demonstrate that the observed phenotypes were due to the um03848 mutation, we examined whether this phenotype was reversed when the wild type copy of $u m 03848$ gene was introduced into the $1 / 2$ $\Delta u m 03848$ mutant. We also constructed a $u m 03848$ overexpressor strain by an ectopic addition of the um03848 gene in the wild type strains. The complementation of um03848 in $\Delta u m 03848$ was not able to complement the growth defect on nitrate medium, but partially reversed the defect in virulence. Overexpression of the um03848 gene in $U$. maydis showed similar growth ability and similar virulence as that of the wild type strain.

\section{Methods}

Strains and growth conditions. The $U$. maydis strains used in this study are listed in Table 2.1. Escherichia coli strain DH5 $\alpha$ (Thermo Fisher) was used for all cloning and plasmid maintenance. U. maydis strains were grown at $27^{\circ} \mathrm{C}$ in solid or liquid YEPS, PDA, HSS media (Brachmann, König, Julius, \& Feldbrügge, 2004) or Holliday Salt 
media (Holliday, 1974). Strains grown in liquid media were grown overnight, shaking at $250 \mathrm{rpm}$. E. coli cells were grown in LB medium, supplemented with $200 \mu \mathrm{g} / \mathrm{mL}$ ampicillin if plasmid selection was required, at $37^{\circ} \mathrm{C}$.

For the determination of nitrogen utilization, minimal medium (MM) plates ${ }^{98}$ were supplemented with $2 \%(\mathrm{wt} / \mathrm{vol})$ glucose and $0.3 \%(\mathrm{wt} / \mathrm{vol}) \mathrm{KNO}_{3},\left(\mathrm{NH}_{4}\right)_{2} \mathrm{SO}_{4}(\mathrm{AMM})$, or $10 \mathrm{mM}$ Urea; for nitrogen starvation medium $(-\mathrm{N})$, no nitrogen source was added, as described previously ${ }^{6}$. For liquid medium, agar was omitted. Mating media was made with solid PDA and 1\% activated charcoal, while stress media were made with solid YEPS and either Congo red (Fisher Scientific, Fairlawn, NJ, $15 \mu \mathrm{g} / \mathrm{ml}$ ), sodium chloride $(1 \mathrm{M})$, or sorbitol $(1 \mathrm{M})$ as described ${ }^{110,111}$.

Table 2. 1 Strains used in Chapter II.

\begin{tabular}{|c|c|c|}
\hline Ustilago Strain & Genotype & Reference \\
\hline $1 / 2$ & $a l b l$ & Gold et al., 1997 \\
\hline $2 / 9$ & $a 2 b 2$ & Gold et al., 1997 \\
\hline $1 / 2 \Delta u m 03848-H y g R$ & albl um03848::hyg ${ }^{R}$ & This study \\
\hline 2/9 $\Delta u m 03848-H y g R$ & $a 2 b 2$ um03848::hyg ${ }^{R}$ & This study \\
\hline $1 / 2 \Delta u m 03848-C b x R$ & alb1 um03848::cbx $x^{R}$ & This study \\
\hline 2/9 $\Delta u m 03848-C b x R$ & $a 2 b 2$ um03848::cbx $x^{R}$ & This study \\
\hline $1 / 2 \Delta u m 03848 c$ & alb1 um03848:: hyg $g^{R}$ & This study \\
\hline 2/9 $\Delta u m 03848 c$ & $\begin{array}{l}\text { Potef-um03848,cbx } \\
\text { a2b2 um03848:: hyg } \\
\text { Potef-um03848,cbx }\end{array}$ & This study \\
\hline $1 / 2$ um03848 $8^{\text {Otef }}$ & alb1 $P_{\text {otef-um03848, } c b x^{R}}$ & This study \\
\hline $2 / 9$ um03848 & $a 2 b 2 P_{\text {otef- }}-u m 03848, c b x^{R}$ & This study \\
\hline SG200 & $a 1 m f a 2 b W 2 b E 1$ & Kämper J. et al., 2006 \\
\hline SG200 $\Delta u m 03848$ & $a 1 m f a 2 b W 2 b E 1$ um03848::hyg ${ }^{R}$ & This study \\
\hline D132 & $a 1 a 2 b 1 b 2$ & Kronstad and Leong, 1989 \\
\hline D132 $\Delta u m 03848$ & $\begin{array}{l}\text { ala2b1b2 um03848 } \\
\text { um03848:: hyg }\end{array}$ & This study \\
\hline
\end{tabular}


Primer design and PCR. Primers (Table 2.2) were designed using Primer 3

(http://bioinfo.ut.ee/primer3-0.4.0/) ${ }^{112}$ on the genome sequence of $U$. maydis available at Joint Genome Institute, JGI (https://genome.jgi.doe.gov/pages/search-for-

genes.jsf?organism=Ustma1). Primers were obtained from Eurofins MWG Operon USA.

PCR was carried out in a T100 Thermal Cycler (Bio-Rad Laboratories, Hercules, CA).

For PCR amplification reactions, either Ex-Taq Hot Start DNA polymerase, PrimeSTAR

Max HS DNA Polymerase (Takara Bio, USA) or DreamTaq Hot Start DNA Polymerase

(Thermo Fisher Scientific, USA) was used. PCR cycling conditions utilized an initial

denaturation temperature of $94{ }^{\circ} \mathrm{C}$ for 4 minutes, followed by 34 cycles of a three-step

process with denaturation at $94{ }^{\circ} \mathrm{C}$ for 30 seconds, annealing at $60{ }^{\circ} \mathrm{C}$ for 30 seconds and

extension at $72{ }^{\circ} \mathrm{C}$ for 1 minute per $1 \mathrm{~kb}$ of anticipated product length, and final extension

at $72{ }^{\circ} \mathrm{C}$ for 10 minutes.

Table 2. 2 List of primers for PCR for Chapter II.

\begin{tabular}{ll}
\hline Primers & Sequence $\left(\mathbf{5}^{\prime} \rightarrow \mathbf{3}\right.$ ') \\
\hline um03848UpFIF & CTTTCAAGTCGTCCCCTTT \\
um03848UpRtHyg & GTAGTTACCACGTTCGGCCATGAATCTCTGTTCCGCTTGG \\
HygLftum03848T & CCAAGCGGAACAGAGATTCATGGCCGAACGTGGTAACTAC \\
HygRtum03848T & AAGAAATCGGTCAAAGCCCTTCAGGCCTCATGTTTGACAG \\
um03848DnFlFHygT & CTGTCAAACATGAGGCCTGAAGGGCTTTGACCGATTTCTT \\
um03848DnRt & GCCTCACATTGCCTACAACC \\
New 3848T-AmpFw & GCAATGTGAGGCTTAATTAATATTGAAAAAGGAAGAG \\
AmpOriR 3848T & GACTTGAAAGGATATCAAAAGGCCGCGTTGC \\
um03848UpF-OriT & CCTTTTGATATCCTTTCAAGTCGTCCCCCTTT \\
um03848UpR-CbxT & CATCTAGGCCATGAATCTCTGTTCCGCTTGG \\
CbxF-um03848T & AGAGATTCATGGCCTAGATGGCCACCATGG \\
CbxR-um03848T & TCAAAGCCCTGGCCACTCAGGCCTATTAAT \\
um03848DnF-CbxT & CTGAGTGGCCAGGGCTTTGACCGATTTCTT \\
New AmpT 3848DnR & CCTTTTCAATATTAATTAAGCCTCACATTGCCTACAACCG \\
um03848qRTLft & TCTGGCTGCGTTCGTGAGT \\
um03848qRTRt & TGGTGGCGATAAGTCCAAAGT \\
um3848ovFw BamHI & acaacatcatccacgGGATCCGCTGAAACGTAAAGCGAACC
\end{tabular}


um3848ovxR NotI um03848cFw-SbFI um3848cR-BamHI
gatctgcagccgggcggecgcATCTTATCCGCTGCGTCAAA gaagcttgcatgcctgcaggCTTTCAAGTCGTCCCCCTTT cggccgttactagtggatccGCCTCACATTGCCTACAACC

Genetic manipulation. The deletion of um03848 in U. maydis was obtained by homologous recombination as described previously ${ }^{113}$. The deletion construct for um03848 was created in a two-step process. First, the $1 \mathrm{~kb}$ flanking region upstream and downstream of um03848 gene from wild type 1/2 strain and the hygromycin resistance cassette from plasmid pUMa1507 ${ }^{114}$ or the carboxin resistance cassette from plasmid pOtefCbxRGFP, were amplified using primers listed in table 2.2. Secondly overlap-PCR was performed as described previously ${ }^{115}$. The merged construct, either flanks with HygR or flanks with CbxR, was then transformed into U. maydis strains by the polyethylene glycol/ protoplast method ${ }^{113,116}$ which replaces the um03848 ORF with hygromycin resistance cassette or carboxin resistance (Figure A.9). Complementation and overexpression constructs were produced using the Gibson assembly technique ${ }^{117}$. First, the $u m 03848$ gene along with the terminator was amplified using um03848ovx Forward and Reverse primers. The plasmid pOtefCbxRGFP was digested using endonucleases BamHI and NotI (New England Biolabs Inc.). Digested plasmid was then ligated with PCR amplified um03848 gene using Gibson assembly technique. Finally, the constructed p3848Otefovx plasmid was transformed into commercially competent $E$. coli DH5 $\alpha$. The transformed colonies were grown in selective medium and the isolated plasmids sequenced using different primers to make sure the plasmid has um03848 gene under expression of pOtef promoter. The sequence confirmed the plasmid was digested with endonuclease SspI which digests within the ip $(\mathrm{CbxR})$ gene and creates a sequence 
homologous to the corresponding region for the sensitive wild type variant of the gene (encoding succinate dehydrogenase) in U. maydis. The digested linear plasmid was then transformed into wild type (WT) and $\Delta u m 03848$ strains to obtain overexpression and complementation strains, respectively. Transformed $U$. maydis were selected on YEPS agar plates containing $3 \mu \mathrm{g}$ carboxin per ml (Santa Cruz Biotechnology, Dallas, TX). Successful transformation was confirmed by PCR and Real time quantitative PCR (qRTPCR) using the primers that would distinguish the transformants from the respective WT strains. The qRT-PCR on complementation strains showed similar gene expression to their respective WT strains, indicating that the um03848 gene has been complemented in these strains. The overexpression and complementation strains showed significant expression of um03848 gene in 1/2 and 2/9 mating strains (Figure A.9).

RNA isolation and expression analysis. RNA isolation from $U$. maydis strains and quantitative real-time PCR were carried out as previously described ${ }^{65}$. For RNA isolation, $U$. maydis cells were grown on high ammonium and low ammonium agar plates for 48 hours. The cells were scraped off the plates and homogenized in a frozen mortar and pestle. Total RNA from homogenized cells was isolated using the TRIzol reagent protocol from Invitrogen. Double stranded cDNA samples were prepared with Super Script IV [Invitrogen, Carlsbad, CA] following the manufacturer's instructions and using $2.5 \mu \mathrm{g}$ RNA as the starting material. To evaluate the expression levels of gene specific primers (Table 2.3) were designed using the ABI Primer Express software version 3.0. The $12 \mathrm{X}$ diluted cDNA was used as a template for real time quantitative RT-PCR (qRTPCR) in a $32 \mu 1$ mixture containing $6.5 \mu 1$ of EvaGreen (Mango Biotechnology, Mountain View, CA), diluted cDNA preparation, $0.8 \mu$ l of each $2.5 \mu \mathrm{M}$ forward and 
reverse primers. Quantitative real time PCR reactions were performed in an Applied Biosystems Step-One thermocycler set for $95{ }^{\circ} \mathrm{C}$ for 10 mins followed by 40 cycles of 95 ${ }^{\circ} \mathrm{C}$ for 15 secs and $60{ }^{\circ} \mathrm{C}$ for $1 \mathrm{~min}$. The real time PCR assay is detected by accumulation of a fluorescent signal and determined by comparative $\mathrm{Ct}$ values. The cycle threshold $(\mathrm{Ct})$ value of a reaction is defined as the number of cycles required for the fluorescent signal to cross the threshold or background level. The $\mathrm{Ct}$ values for gene expression were normalized to the expression of the constitutively expressed gene, eif $2 B$, encoding the translation initiation factor eIF2. Changes in the gene expression are the averages of three technical triplicates and three biological replicates and expressed in $\log 2$-fold changes relative to expression of WT grown in similar condition. RNA expression data were normalized to the expression of respective strains under high ammonium conditions. Data were analyzed in GraphPad Prism 7.0 using One-way ANOVA followed by Tukey's multiple comparison. A probability value of $\mathrm{p}<0.05$ was considered to be significant.

Table 2. 3 Genes and primers used for qRT-PCR for Chapter II

\begin{tabular}{|c|c|c|}
\hline Gene name & Primers & Sequence $\left(5^{\prime} \rightarrow 3^{\prime}\right)$ \\
\hline \multirow{2}{*}{$\begin{array}{l}\text { Guanine nucleotide } \\
\text { exchange factor }\end{array}$} & rt-eIF-2B-F new & ATCCCGAACAGCCCAAAC \\
\hline & rt-eIF-2B-R new & ATCGTCAACCGCAACCAC \\
\hline \multirow[t]{2}{*}{ prfl (um02713) } & rt-Prf1-F new & TCGGTAGAACGAGCTGTGATG \\
\hline & rt-Prf1-R new & CTGTTGGACGATGTTGGAGTTG \\
\hline \multirow[t]{2}{*}{$r b f 1$} & RT Rbf1 F & AGGGTGTGGCAAATCGTTCT \\
\hline & RT Rbf1 R & TCGGCATCAGCATGGTTTC \\
\hline \multirow{4}{*}{$m f a(\mathrm{um} 02382)$} & rt-Mfa1-F & ATGCTTTCGATCTTCGCTCAG \\
\hline & Rt-Mfa1-R & TAGCCGATGGGAGAACCGT \\
\hline & Mfa2 F qrt & AGCAGGCTTCCAACAACGA \\
\hline & Mfa2 R qrt & CACATCTAGGCGATCAAGCAGTA \\
\hline \multirow{4}{*}{$b E(\mathrm{um} 00577)$} & b1Elft123 & GCAACAAAAGATACCCAACGA \\
\hline & b1Ert334 & TTCGACACCCTACATCAGGAC \\
\hline & new bE2q1277-F & CAGGGGCAATAGGAAAGTCA \\
\hline & new bE2q 1330-R & ACCATTTTCGACCTCGTCAG \\
\hline$b W($ um00578) & b1Wlft1559 & TCGAGTCTGCCTCAATTCCT \\
\hline
\end{tabular}




\begin{tabular}{lll} 
& b1Wrt1827 & CTCTCCTATGCTGGCTCCAC \\
& bW2 F qrt & CGTGGAGCCTACGGAATCA \\
& bW2 R qrt & GGAGGTGACTCGTGTCTGGAA \\
\hline ump2 (um05889) & rt-ump2-F & TGGGTCCCGTTCTCATTTTC \\
& rt-ump2-R & AGGCGATGGGATTGTAGACAA \\
\hline
\end{tabular}

Nitrogen utilization, stress and mating assay. For nitrogen utilization, stress and mating assays, strains were grown in $4 \mathrm{~mL}$ YEPS liquid medium shaken at $280 \mathrm{rpm}$ until an absorbance at $600 \mathrm{~nm}\left(\mathrm{OD}_{600}\right)$ of 0.6-0.8. The liquid culture was collected, cells were washed and resuspended in water. The strains were then diluted with water up to $10^{5}$ fold. Then, $10 \mu \mathrm{l}$ of each diluted and undiluted strains was spotted onto nitrogen utilization and stress media ${ }^{111}$. Mating assays were performed using strains grown overnight in YEPS broth to an $\mathrm{OD}_{600}$ of 0.6-0.8. Strains were collected and resuspended in water to an $\mathrm{OD}_{600}$ of 2.0. Opposite mating-type strains were co-spotted $(10 \mu \mathrm{l})$ onto PDA $1 \%$ charcoal plate and the mating was observed after incubation at $28{ }^{\circ} \mathrm{C}$ for $24-48$ $\mathrm{h}$ by the presence of white $f u z z$ as evidence of successful mating ${ }^{78,118}$.

Cell growth rate and infection assay. For cell growth rate, the cells were grown in YEPS liquid medium overnight to reach exponential growth phase. Cells were then collected by centrifugation, washed and resuspended in $50 \mathrm{~mL}$ fresh liquid media of complete medium (CM) or minimal medium (MM). After $8 \mathrm{~h}$ of incubation at $28{ }^{\circ} \mathrm{C}$ with $250 \mathrm{rpm}$ shaking, the optical density was determined every 2 hours up to $30 \mathrm{~h}^{47}$. Three biological replicates of this growth process were performed. Data were analyzed using GraphPad Prism.

For the plant infection assay, strains grown in $20 \mathrm{ml}$ YEPS media up to $\mathrm{OD}_{600}$ of 1.0 were collected and resuspended in water to concentrate up to an $\mathrm{OD}_{600}$ 3.0. Organic 
Golden Bantam (Territorial Seed Company) corn seedlings (8-10 days old) were injected with pre-mixed haploid strains of opposite mating type or directly for the solopathogenic SG200 strain and diploid D132 strain, or with water (mock), as previously described ${ }^{78}$. The virulence symptoms were rated on 8- and 14- days post infection (dpi) by disease index (DI) on a scale of 0 to 5 , where $0=$ No symptoms, $1=$ Chlorosis, $2=$ Small leaf tumors, $3=$ medium leaf tumors or small leaf tumors in multiple leaves, $4=$ stem tumor and $5=$ dead ${ }^{78}$. Additional measures of virulence included the difference in number of leaves and mean difference in the plant length, measured between the day of infection and 8 dpi.

Statistical analysis of the disease index measures was performed using a KruskalWallis Test with Multiple Comparison Test using the $\mathrm{R}$ program ${ }^{119,120}$. The mean plant length difference and mean difference in number of leaves ( $8 \mathrm{dpi}-0 \mathrm{dpi})$ were analyzed using ANOVA followed by Tukey's test comparison across the groups ${ }^{121}$ using GraphPad Prism. The probability value, $\mathrm{p}<0.05$ was considered significant.

\section{Results}

\section{Analysis of um03848 showed high sequence similarity to known nitrite reductase}

from other filamentous fungi. The initial analysis of the genome of $U$. maydis suggested that um03848 (UMAG_11104) is a potential nitrite reductase ${ }^{55}$. To find the sequence similarity with the known nitrite reductases from other filamentous fungi, BLASTp ${ }^{122}$ was performed. BLASTp was done on translated um03848 protein against the protein sequences of known nitrite reductases from other filamentous fungi such as Aspergillus nidulans and Fusarium oxysporum and the fungi belonging to the order 
Ustilaginales, Moesziomyces aphidis (old name- Psedudozyma aphidis). The nitrite reductase protein has been identified in these fungi based on bioinformatic ${ }^{123,124}$ and mutational analyses ${ }^{100,125}$. BLASTp showed high sequence similarity of $U$. maydis um03848 with known nitrite reductases from fungal orthologs. In these alignments, um03848 (602 amino acid residues) showed about 90\% of total amino acid sequence similarity with the ortholog of the closely related fungus M. aphidis (1248 amino acids) with $0 \%$ gap. Whereas, it showed $57 \%$ similarity with $2 \%$ gap and $54 \%$ similarity with 4\% gap to the total amino acid sequences of filamentous fungi A. nidulans (1104 amino acids) and F. oxysporum (1089 amino acid), respectively (Table 2.4, Figure 2.1 A). Similarly, um03848 protein sequence was analyzed with the NCBI conserved domain analysis tool (https://www.ncbi.nlm.nih.gov/Structure/cdd/wrpsb.cgi) ${ }^{109}$. The U. maydis um03848 protein is characterized by conserved PRK14989 super family nitrite reductase $[\mathrm{NAD}(\mathrm{P}) \mathrm{H}]$ and Rieske-like [2Fe-2S] domain (Figure 2.1 B).

Table 2. 4 Summary of BLASTp analysis for um03848 with identified nitrite reductase.

\begin{tabular}{lcccccc}
\hline \multicolumn{1}{c}{ Species } & Gene ID & $\begin{array}{c}\text { Max } \\
\text { Score }\end{array}$ & $\begin{array}{c}\text { Total } \\
\text { Score }\end{array}$ & $\begin{array}{c}\text { Query } \\
\text { Cover }\end{array}$ & $\begin{array}{c}\text { Per. } \\
\text { Ident }\end{array}$ & $\begin{array}{c}\text { E- } \\
\text { value }\end{array}$ \\
\hline Moesziomyces aphidis & ETS64086.1 & 1136 & 1201 & $100 \%$ & $89.37 \%$ & 0.0 \\
\hline Aspergillus nidulans & CBF88349.1 & 651 & 729 & $90 \%$ & $57.38 \%$ & 0.0 \\
\hline Fusarium oxysporum & BAF31848.1 & 612 & 650 & $90 \%$ & $54.19 \%$ & 0.0 \\
\hline
\end{tabular}




\begin{tabular}{|c|c|c|c|c|}
\hline \multicolumn{5}{|l|}{ (A) } \\
\hline$\square$ Query_33613 & 1 & \multicolumn{2}{|c|}{ MKSSTKCGTGCGGCMPLST } & 19 \\
\hline & 483 & \multicolumn{2}{|c|}{ LCNKGAAIEKPPAELIIGAKKEGEEEGDDLPDEAQVCSCHNVTKGDLIRCVKDGGVRSVGEMKSSTKCGTGCGGCLPLSQ } & 562 \\
\hline$\square \overline{\mathrm{CBF} 88349.1}$ & 464 & \multicolumn{2}{|c|}{ MVKSQKPLEVPPSEFILGAQSGGEENADDLDDSTQICSCHNVTKGDVVESVKSGTCKTIADVKSCTKAGTGCGGCMPLVQ } & 543 \\
\hline$\square \underline{B A F 31848.1}$ & 423 & \multicolumn{2}{|c|}{ MVKAQKELEISPSELILGAKKDGGEDTDDLDDDAQVCSCHNVTKGDIVKVVKDGTCKDVASIKKCTKAGTGCGGCVPLIT } & 502 \\
\hline$\square$ Query_33613 & 20 & \multicolumn{2}{|c|}{ SILNKALKEAGVEVSNHLCGHFAYSRQDLYQIIKFKKLKDFTTVMSTVGKKADSLGCEVCRPAIGSILSSLYNDWIMAPQ } & 99 \\
\hline$\square$ ETS64086.1 & 563 & \multicolumn{2}{|c|}{ SILNKALKEAGVEISNHLCPHFAYTRQELYQIIKFKKLKDFTSAMQAVGKKADSLGCEVCRPAVGSILSSLYNDWIMNPQ } & \\
\hline$\square \mathrm{CBF88349.1}$ & 544 & \multirow{2}{*}{\multicolumn{2}{|c|}{$\begin{array}{l}\text { SIFNKTMLDMGQEVSNNLCVHIPYSRADLYNVIAIRQLRTFDDVMKSAGKCPDSLGCEICKPAIASILSSLFNPHLMDKE } \\
\text { TIFNKTMASMGQEVTNYVCSHFNHSRADLFNIIMVKRLKNMGEVMREAGNDKEALGCELCKPVVASIMASLWNRHVMDKT }\end{array}$}} & 623 \\
\hline$\square$ BAF31848.1 & 503 & & & 582 \\
\hline$\square \mathrm{Qu}$ & 100 & \multicolumn{2}{|c|}{ LRQTQDTNDRYLANMQRDGTYSVVPRLAAGEITPAGLKVIGEVAEEYGLYTKITGGQRIDMFGAKKQDLPAIWTKLVDAG } & 179 \\
\hline VETS64086.1 & 643 & \multirow{2}{*}{\multicolumn{2}{|c|}{ QRQTQDTNDRYLANVQRDGTYSVVPRLPAGEVTPAGLKVIGEVAEEYGLYTKITGGQRIDMFGAKKQDLPAIWEKLVNAG }} & 722 \\
\hline$\square \underline{\mathrm{CBF} 88349.1}$ & 624 & \multirow{2}{*}{\multicolumn{2}{|c|}{$\begin{array}{l}\text { YHELQETNDRFLANIQRNGTFSVVPRVPGGEITADKLIAIGQVAKKYNLYCKITGGQRIDMFGARKQDLLDIWTELVDAG } \\
\text { THGLQETNDRFLGIQRDGTYSVVPRVSGGEITPDKLVVIGEVAQKYNLYTKITGGQRIDLFGAQKQDLLDIWGQLVAGG }\end{array}$}} & \\
\hline$\square$ BAF31848.1 & 583 & & & 662 \\
\hline$\square \mathrm{Qu}$ & 30 & \multicolumn{2}{|c|}{ FESGHAYGKALRTVKSCVGSTWCRYGVGDSVGLAHELETRYKGIRAPHKFKGGISGCVRECAEAQGKDFGLIATTKGWNV } & 259 \\
\hline ఐETS64086.1 & 723 & \multicolumn{2}{|c|}{ FESGHAYGKALRTVKSCVGSTWCRYGVGDSVGLAHELETRYKGLRAPHKFKGGVSGCVRECAEAQGKDFGLIATTKGWNV } & 802 \\
\hline$\square \overline{\mathrm{CBF} 88349.1}$ & 704 & \multirow{2}{*}{\multicolumn{2}{|c|}{$\begin{array}{l}\text { MESGHAYAKSLRTVKSCVGTTWCRFGVGDSVGMAIRLEQRYKSIRAPHKFKGAVSGCVRECAEAQNKDFGLIATEKGFNI } \\
\text { MESGHAYAKSLRTVKSCVGSTWCRFGLSDSVGMAVRLEERYKSIRAPHKIKGGVSGCVRECAEAQGKDFGLIATEKGWNI }\end{array}$}} & \\
\hline$\square \overline{\mathrm{BAF} 31848.1}$ & 663 & & & \\
\hline$\square$ Que & 260 & \multicolumn{2}{|c|}{ YVGGNGGAKPRHAELIAEDVTRRQAIKILDRFILFYIRSADKLERTARWIEKFPGGLKGLKEVIVDDKLGICEDLEKEME } & \\
\hline VETS64086.1 & 803 & \multicolumn{2}{|c|}{ YVGGNGGAKPRHAELIAEDVTRREAIKILDRFILFYIRSADKLERTARWIEKFPGGLKGLKEVIVDDKLGICQDLEREME } & 882 \\
\hline$\square \overline{\mathrm{CBF} 88349.1}$ & 784 & \multirow{2}{*}{\multicolumn{2}{|c|}{$\begin{array}{l}\text { FVGGNGGAKPRHSELLAKDVPPEEVIPILDRYVIFYIRTADKLQRTARWLESLPGGIEYLKDVVLNDKLGIAAEMERQMQ } \\
\text { FVGGNGGANPRHAELLAKDVPPADVVTILDRYLMFYMRTADKLQRTARWIENLPGGIKYLQEVILEDKLGINASLEAQME }\end{array}$}} & \\
\hline$\square \underline{\text { BAF31848.1 }}$ & 743 & & & 822 \\
\hline 613 & 340 & \multicolumn{2}{|c|}{ VLVGTYHCEWTKVVRDPTRHKAFRQFVNTDETQPVSEMMSERDQQRPADWPVETGPLHFSILDVAKEAVWEWRSVCKYTD } & \\
\hline ETS64086.1 & 883 & \multicolumn{2}{|c|}{ VLVGTYHCEWTKVVRDPARQKAFRQFINTDETQEVSEKLSEREQQRPVDWPTESGPLHFSILDVAKEAVWEWRAVCKYSD } & 962 \\
\hline$\nabla \underline{\mathrm{CBF} 88349.1}$ & 864 & \multicolumn{2}{|c|}{ ELVDSYFCEWTETVRNPKRRKYFQQFANTDETVENVEIVKEREQVRPTYWPKDGA-NEDFKG--HQWSSLSWQPVIKADY } & \\
\hline$\square \overline{\mathrm{BAF} 31848.1}$ & 823 & \multicolumn{2}{|c|}{ ELVDSFFDEWAEAIKSPAIAAKFKQFANTNDTTRNMELEDDRGQKRPAFWPADAAATDNFSGLKDKWSMTSWEPIIESSY } & 902 \\
\hline$\checkmark \mathrm{Qu}$ & 420 & \multirow{2}{*}{\multicolumn{2}{|c|}{$\begin{array}{ll}\text { LD--PQKDSPSSATIKYGDTQIAVWNIPGRGVRAAQNMCPPRRAFVLADGLV } & \text { GEDDKGREYVSCPLHKRNYILSAN } \\
\text { LD--PQVDPSSATIKYGDTQIAVWNIPGRGVRAAQNMCPHRRAFVLADGLV } & \text { GEDDKGREYVSCPLHKRNYILSAK }\end{array}$}} & 493 \\
\hline VETS & 963 & & & 103 \\
\hline$\square \underline{\mathrm{CBF} 8}$ & & \multicolumn{2}{|c|}{ F--SDGPPAISSANIKRGDTQLAIFKVKG-KYYATQQMCPHKRTFVLSDGLI GDDDNGKYWVSCPYHKRNFEL--N } & \\
\hline$\nabla \underline{B A F 31848.1}$ & 903 & \multicolumn{2}{|c|}{ FDgADELPNGISATVKRGDTQLAIWRIKG-VYYATQQMCPHKRAFILSDGLI[10]GDKDGASPWVSCPHHKRNFDL --G } & 985 \\
\hline$\checkmark$ Que & 494 & \multicolumn{2}{|c|}{ ADEEGGKCNDGDYSIMTFEAKMDEEKDLVYLKLPPTEALDAVLSTSKWMLRRATEESDAMAGGQSHSVEITGPLDMDTLE } & \\
\hline VETS64086.1 & 1037 & \multicolumn{2}{|c|}{ AEEEGGKCNDGDYSIMTFETKLDKDQDLVFLKLPPTEALDAVLSTSKWMLRRATEESDALAGGQSHSVEITGPLDMDQLE } & 111 \\
\hline$\square \underline{\mathrm{CBF} 88349.1}$ & 1012 & \multicolumn{2}{|c|}{ GEQAGRCQNDEAMNIATFPVEERED-GWIYMKLPPVEELDSVLGTEKWKVKKG-EAVD-PFEAYDKK--YSG---..- } & 107 \\
\hline$\square$ BAF31848.1 & 986 & \multicolumn{2}{|c|}{ N---GACKTDESLSIATFPTEARAD-GMLYLKLPPVEELDAALGTKKWMVKKG-EAGEAPLAKLDSKIKFVG-- } & 105 \\
\hline चQuery_Z & 574 & KGPSSPNKPGSPPAKKTKASCGEQSSLDW--- & 602 & \\
\hline VETS64086.1 & 1117 & SS-SGPKRGSGSVTAPIKASCGE-ASLDWSSM[15]SGGSGGNSADD [76] & 1248 & \\
\hline$\square \mathrm{CBF88349.1}$ & 1079 & $\ldots$ MKGKRAGAKGIEGSKPTRSPSNTI - & 1104 & \\
\hline BAF31848.1 & 1053 & -..-LRGKKPYVKPTGGAKMTTKPLDLMMA & 1089 & \\
\hline
\end{tabular}


(B)

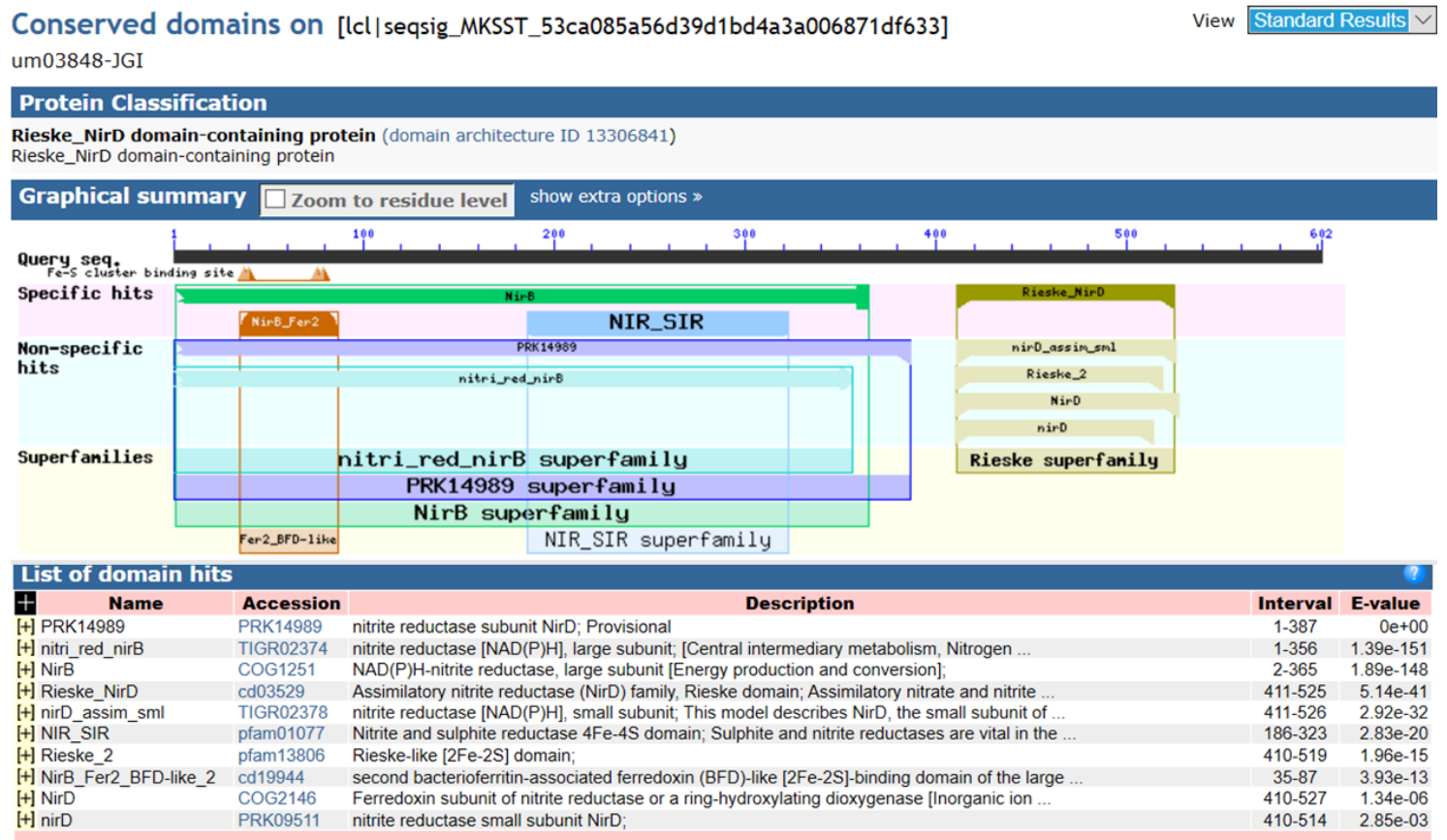

Figure 2. 1 In silico analysis of $U$. maydis nitrite reductase, um03848, protein.

(A) The multiple sequence alignment of $U$. maydis potential nitrite reductase, um03848 (Query) and nitrite reductase from other fungi. Red color shows a high degree of sequence similarity in amino acids at the conserved domains between um03848 and other identified proteins. Blue color shows the difference in amino acid while dash (-) indicates the gap in the alignment. (B) Conserved domain analysis of um03848 in which the graphical summary indicates the presence of different conserved domains in different regions of the query um03848 sequence (black). The table gives the list of domain hits and the description of domain.

Induction of nitrite reductase, um03848, gene in low ammonium. To examine possible induction of nitrite reductase under nitrogen starvation condition, the WT strains were grown on low and high ammonium medium. From the isolated RNA of these strains, qRT-PCR was performed. The expression of the um03848 gene was significantly increased when WT strains (1/2 and 2/9) were grown in ammonium starved condition (Figure 2.2) compared to those grown in high ammonium condition. This observation led 
us to investigate the role of um03848 in the phenotype of $U$. maydis in terms of mating and virulence to its host plant.

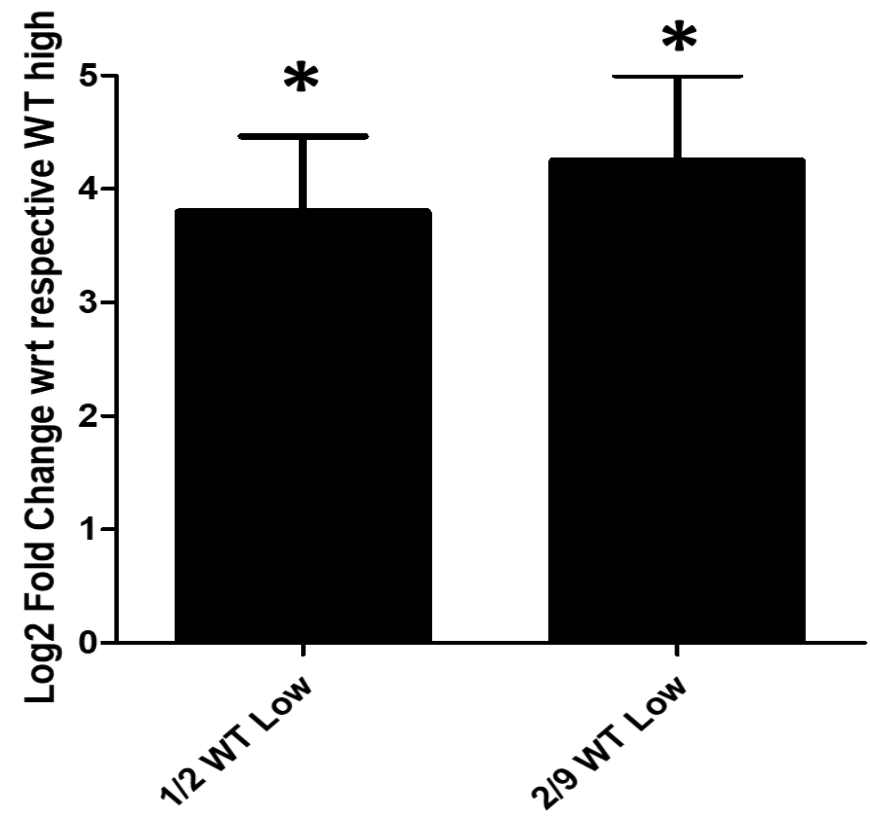

Figure 2. 2 Relative expression of um03848 in wildtype grown under low ammonium conditions.

Bars represent the averages of biological triplicates and standard errors are indicated on top of each bar. Statistical analysis was done using Student's t-test (unpaired two-tailed) in GraphPad Prism 7.0, with respect to (wrt) respective wild type grown on high ammonium, i.e., 1/2 WT low was compared to 1/2 WT on high and 2/9 on low was compared to 2/9 WT grown on high. * indicates the significance with p-value $<0.05$.

\section{Deletion of um03848 does not affect the filamentation of $\boldsymbol{U}$. maydis in low}

ammonium media. The haploid filamentation phenotype was observed for $\Delta u m 03848 . \mathrm{CbxR}$ and $\Delta u m 03848 . \mathrm{HygR}$ of $1 / 2$ and $2 / 9$ strains grown in low ammonium medium for 4 days. Both 1/2 and 2/9 WT background strains showed a characteristic filamentation phenotype when grown on low ammonium medium. We observed similar filamentation on low ammonium medium for all the mutant strains as compared to the wild types (Figure 2.3). 


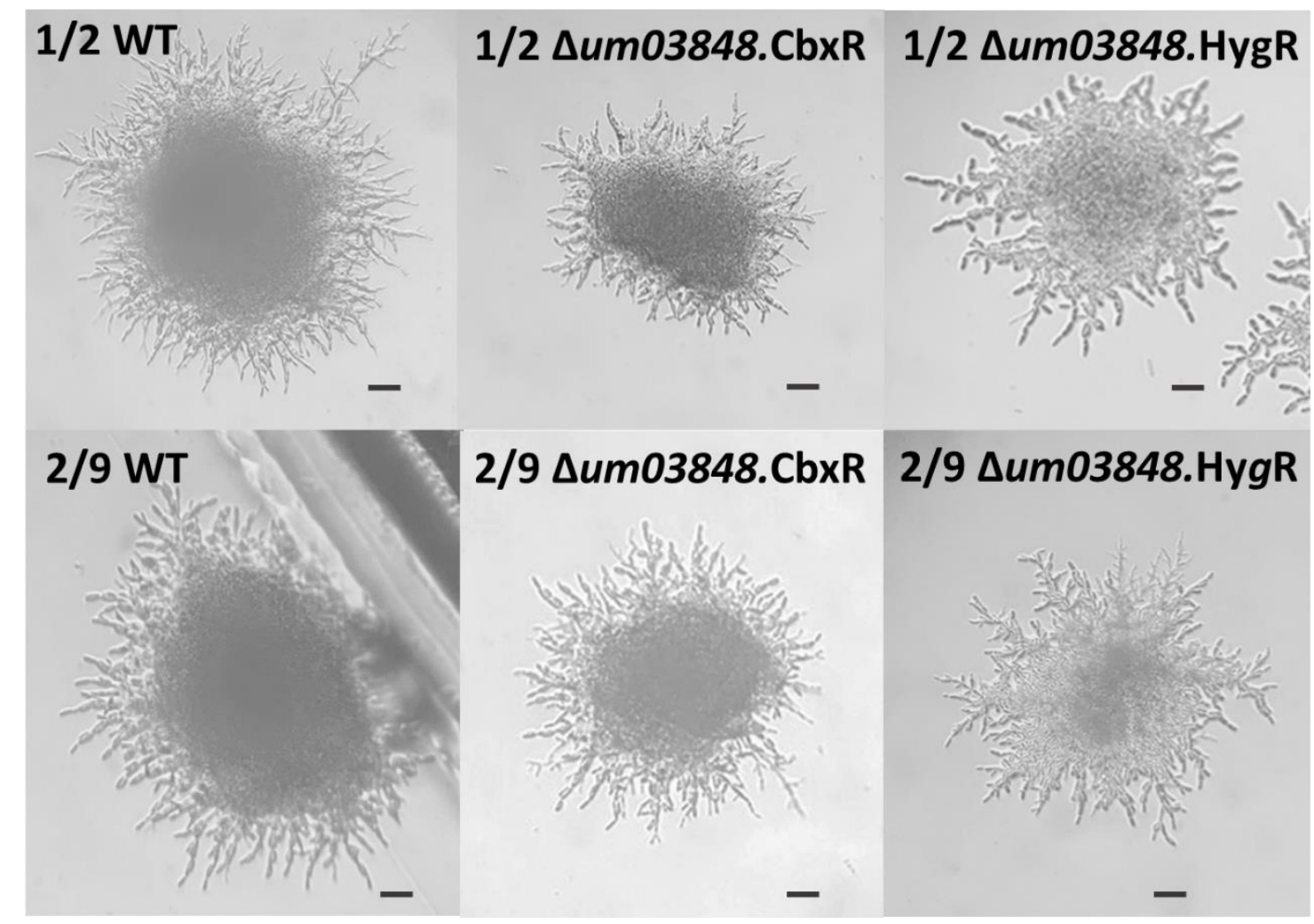

Figure 2. 3 Phenotype associated with um03848 mutants on low ammonium medium. Filamentation assay where haploid cells (labeled) were grown on low ammonium medium. Size bars, $100 \mu \mathrm{m}$.

\section{Deletion of um03848 does not affect the ability of $U$. maydis to mate with its partner.}

To assess the ability to form dikaryotic filament, the mating assay was performed where compatible strains were co-spotted onto PDA charcoal plates. The strains tested were WT (1/2 and 2/9); um03848 deleted mutants ( $\Delta u m 03848 . \mathrm{CbxR}$ and $\Delta u m 03848 . \mathrm{HygR})$; um03848 deletion strains complemented with a functional copy of um03848 locus ( $\Delta u m 03848 \mathrm{c})$, and overexpression of $u m 03848$ with ectopic addition of $u m 03848$ locus introduced into the wild type (um03848 $\left.8^{\mathrm{Otef}}\right)$. Successful mating or aerial hyphae formation is measured as the extent of white "fuzz" growth on charcoal media when observed after $24 \mathrm{~h}$ of incubation. All the mutants showed the characteristic "fuzz" 
phenotype, indicating that the deletion of um03848 does not affect the ability of the fungus to mate with its partner.

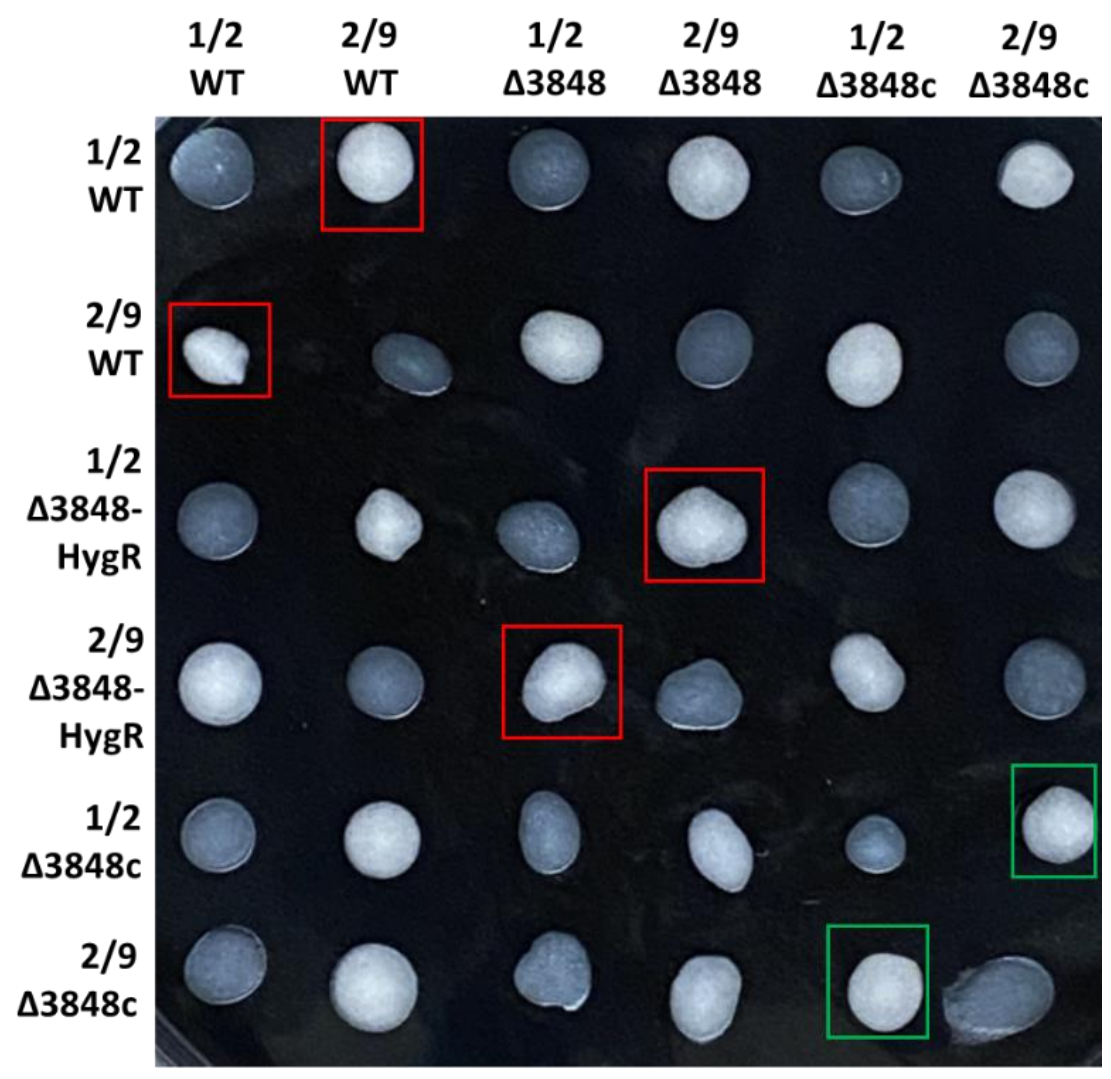




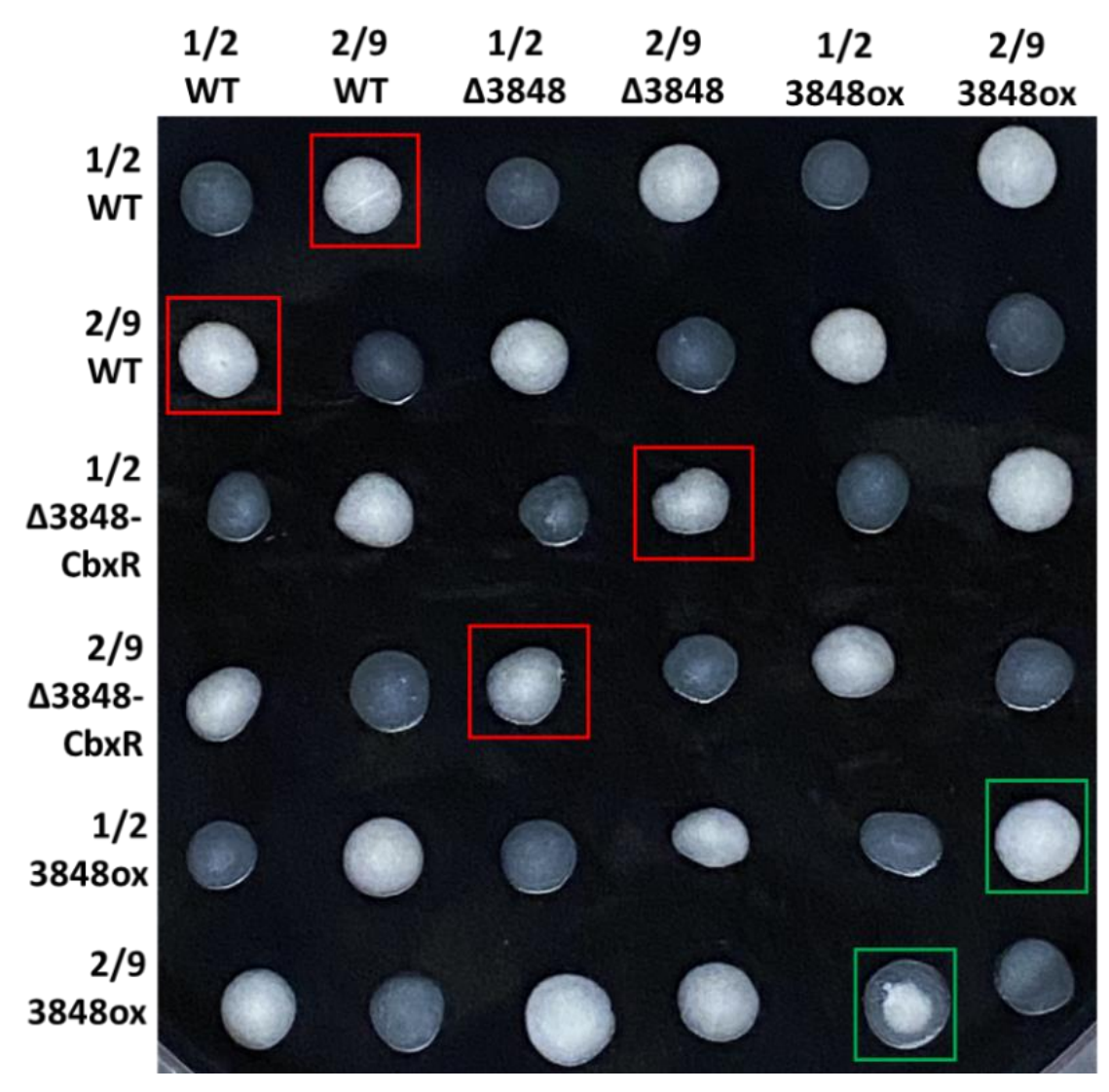

Figure 2. 4 Mating assay on PDA-charcoal plate.

Plate mating assay on $1 \%$ charcoal incorporated PDA plate for $1 / 2$ and 2/9 wild type, corresponding um03848 deletion mutants, these mutants complemented with a wild type copy of um03848 gene. and um03848 over-expressor with ectopic copy of um03848 gene in wild type. Equal mixtures of haploid strains were pre-mixed in a tube and spotted onto PDA plate and grown for $24 \mathrm{hrs}$. Top panel represents mating assay for $\Delta u m 03848$.HygR and complementation ( $\triangle u m 03848 \mathrm{c}$ ) strains and bottom panel is for $\Delta u m 03848 . \mathrm{CbxR}$ and overexpression (3848ox) strains. A positive mating reaction produced a white "fuzz" phenotype of aerial hyphae production. Red boxes indicate the "fuzz" reaction of wild type and $\triangle u m 03848$ with their compatible mating partners. Green boxes indicate "fuzz" reaction by um03848 complementation or over-expressor.

\section{Cell growth of um03848 mutant was not affected in rich medium, while um03848}

\section{was involved in the ability of $U$. maydis to sense and utilize nitrate as a source of}

nitrogen. When um03848 deletion, complementation and overexpression mutants were grown in rich medium such as YEPS, they were able to grow in similar ways as their respective wild type cells (Figure 2.6 A, B). Similarly, in the medium with ammonium or 
urea as a sole source of nitrogen or medium without nitrogen, the growth of the mutants was not affected (Figure 2.5). However, when mutants were grown in medium with nitrate as a sole source of nitrogen, um03848 deletion strains showed a reduced tolerance to nitrate when compared to the respective wild type strains (Figure 2.5 A, B). This was also shown by the time course growth assessment of mutants in liquid medium with nitrate as a source of nitrogen (Figure 2.6 C, D). Although the complemented strains still showed reduced growth on nitrate medium (Figure 2.5 B and 2.6 A), overexpression strains showed improved growth when compared to the respective deleted mutants (Figure $2.5 \mathrm{D}$ ). The $1 / 2$ um03848 overexpression strain grew similar to its respective wild type in nitrate medium, while $2 / 9$ um03848 overexpression strain had reduced growth compared to its wild type. The 2/9 WT showed unexpectedly abundant or normal growth on nitrate medium compared to that of 1/2 WT (Figure 2.5 B, D and Figure 2.6 C, D).

\section{(A)}

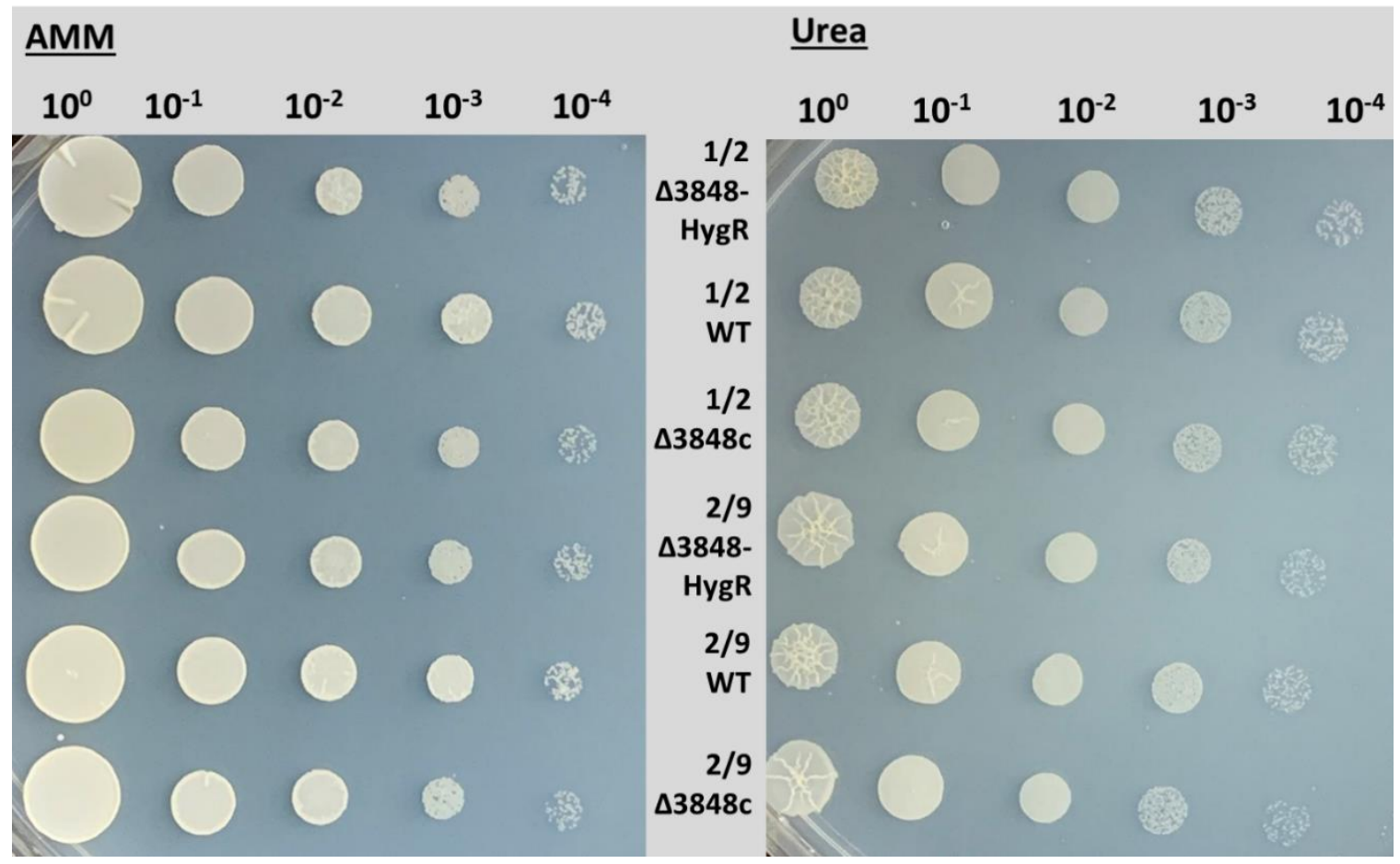


(B)

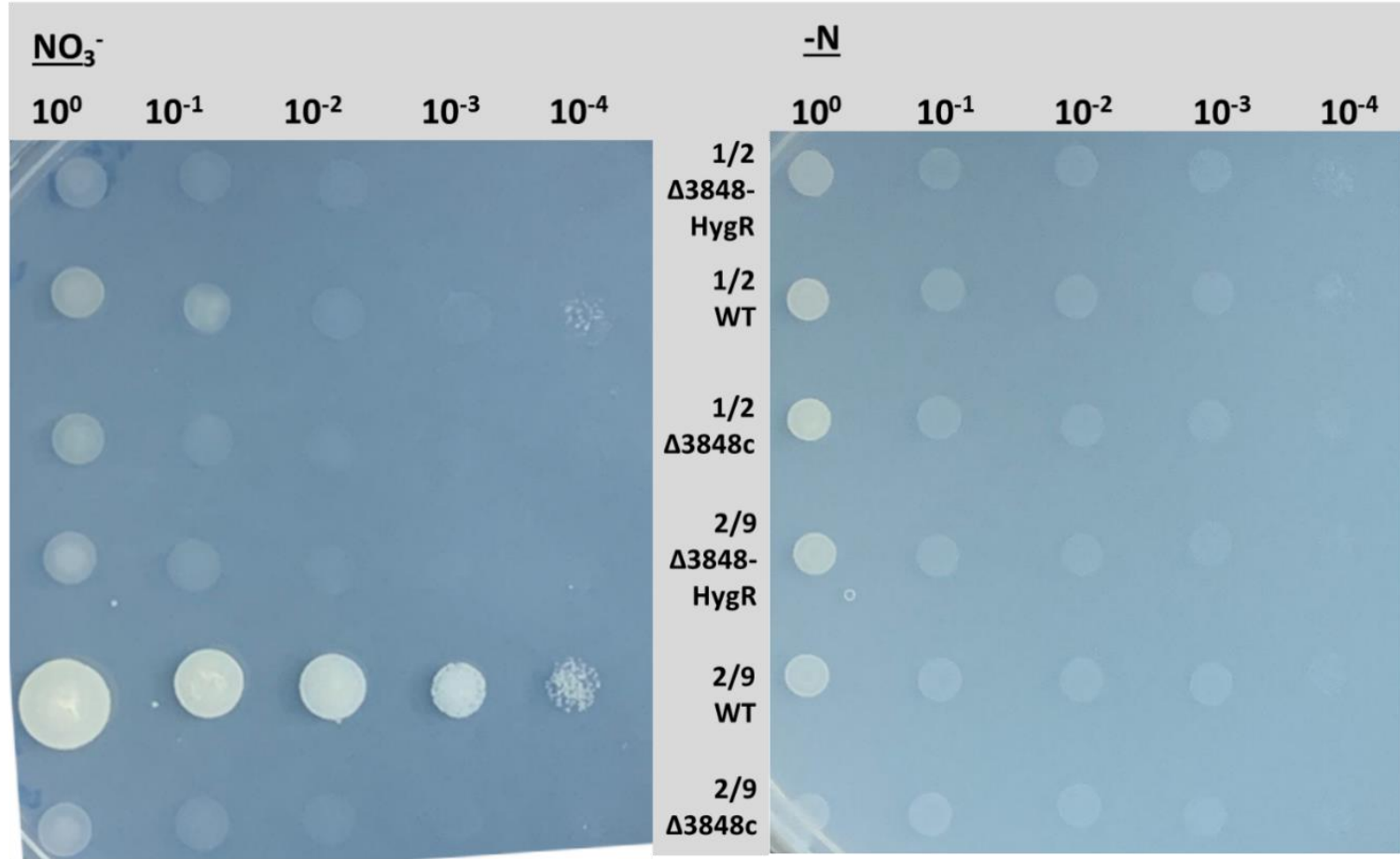

(C)

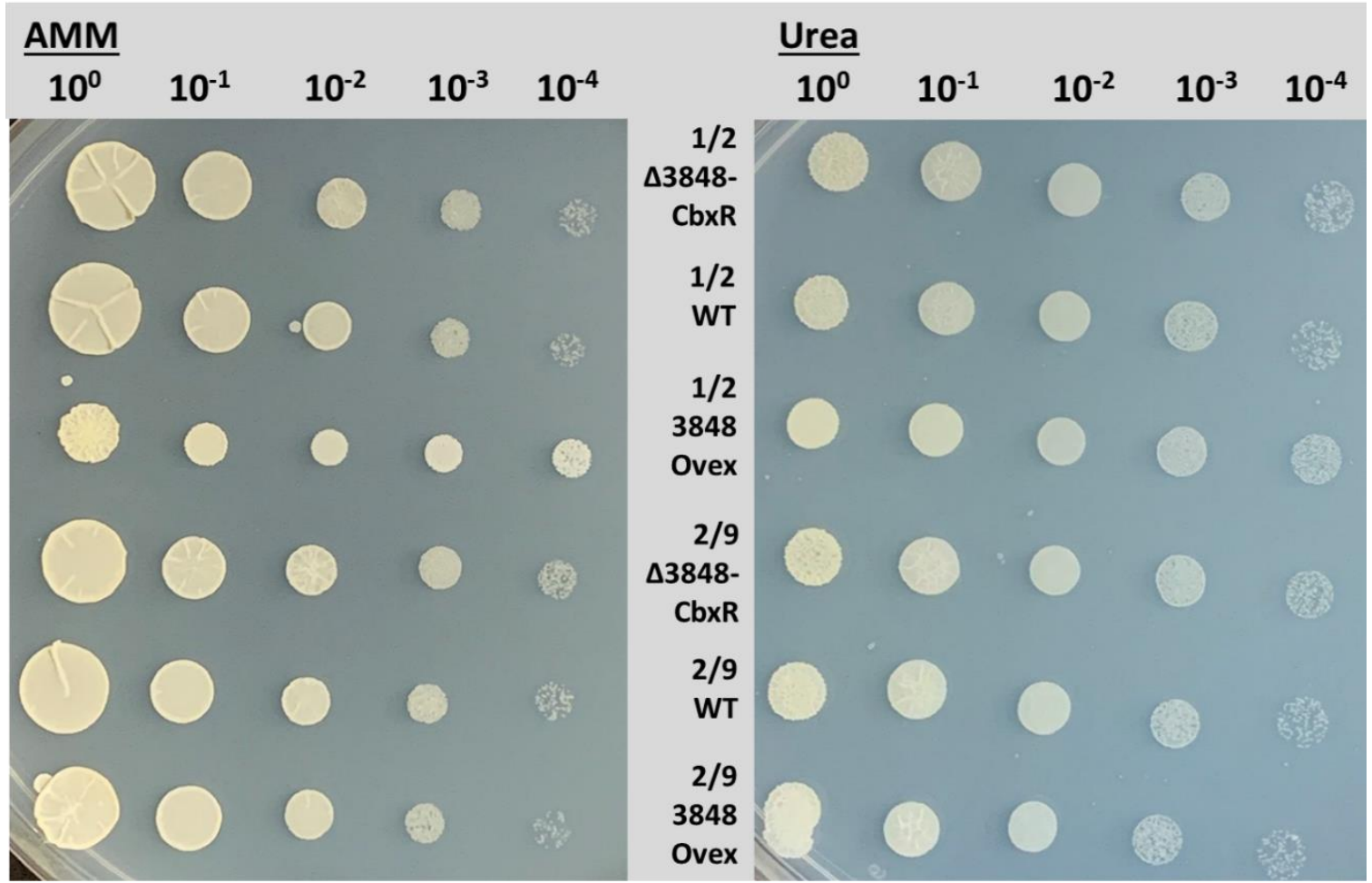




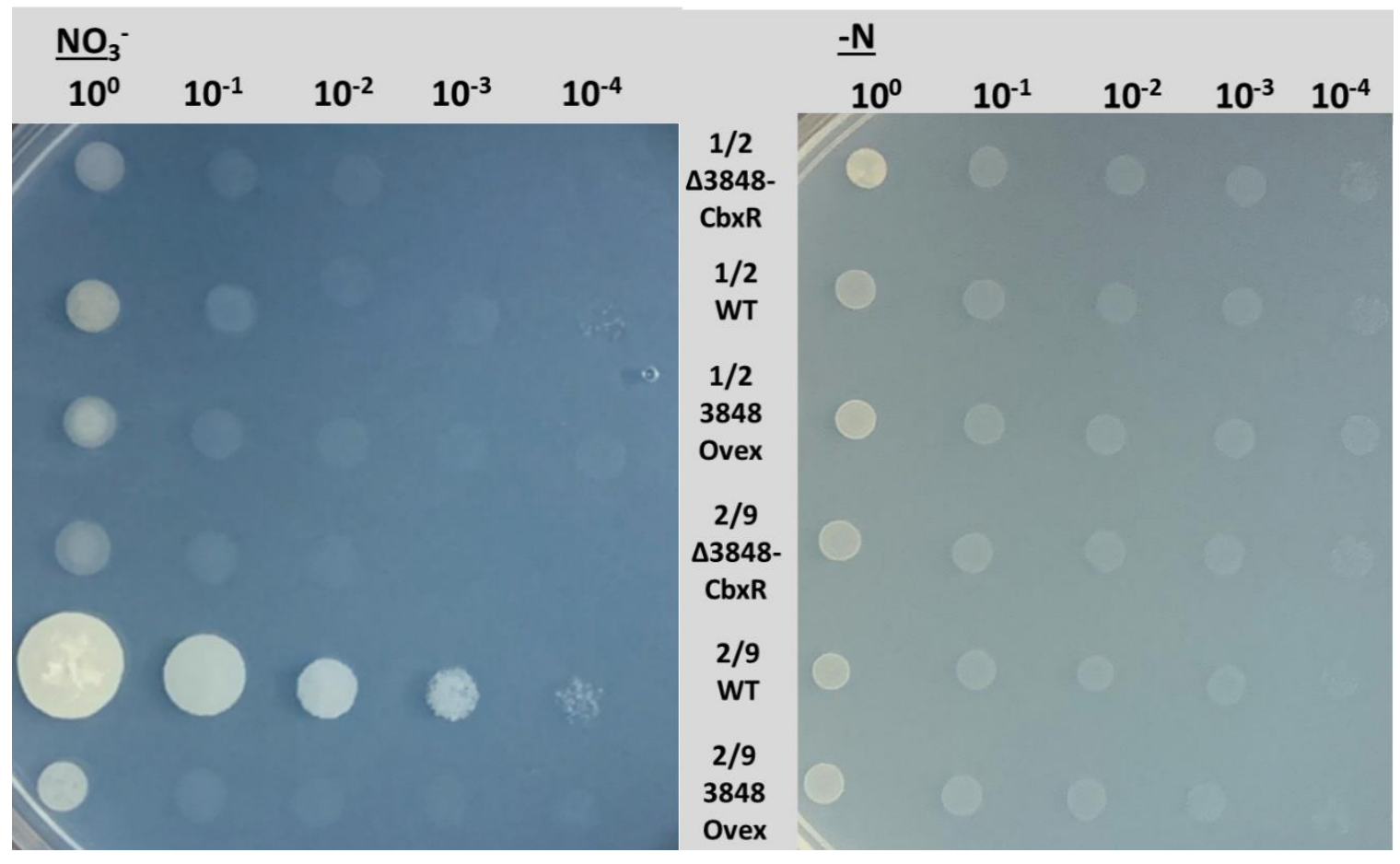

Figure 2. 5 Nitrogen utilization by mutants.

10 -fold serial dilutions of wild types, $\Delta u m 03848, u m 03848 \mathrm{c}$ and $u m 03848^{\text {Otef }}$ strains were spotted onto minimal media with ammonium (AMM), urea, nitrate $\left(\mathrm{NO}_{3}\right)$ and no nitrogen (-N). Plates were grown at $28{ }^{\circ} \mathrm{C}$ for $48 \mathrm{hrs}$. Mutants were compared with their respective wild type. (A, B) $\Delta u m 03848$-HygR and complementation $(\Delta 3848 \mathrm{c})$ are spotted along with respective wild types. (C, D) independently constructed mutant $\triangle u m 03848$-CbxR and overexpression (3848ovex) are spotted along with respective wild types. 


\section{(A) 1/2 in YEPS}

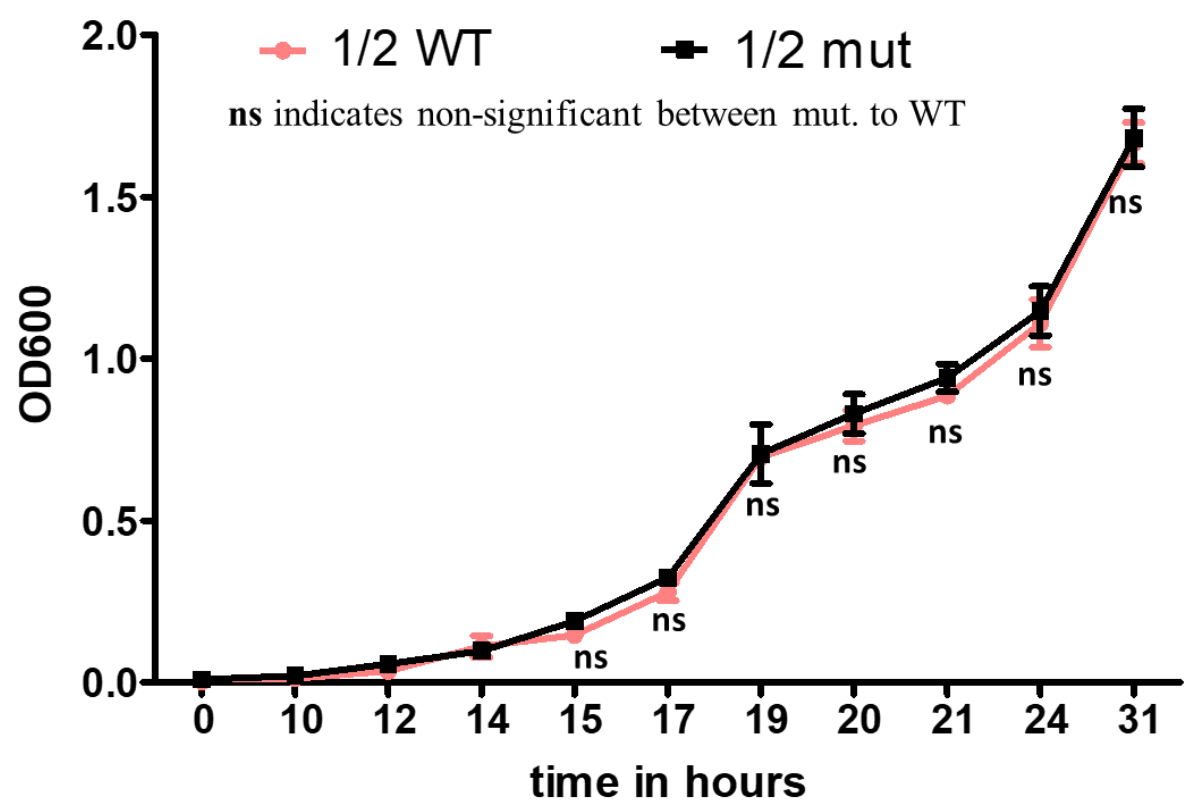

(B) 2/9 in YEPS

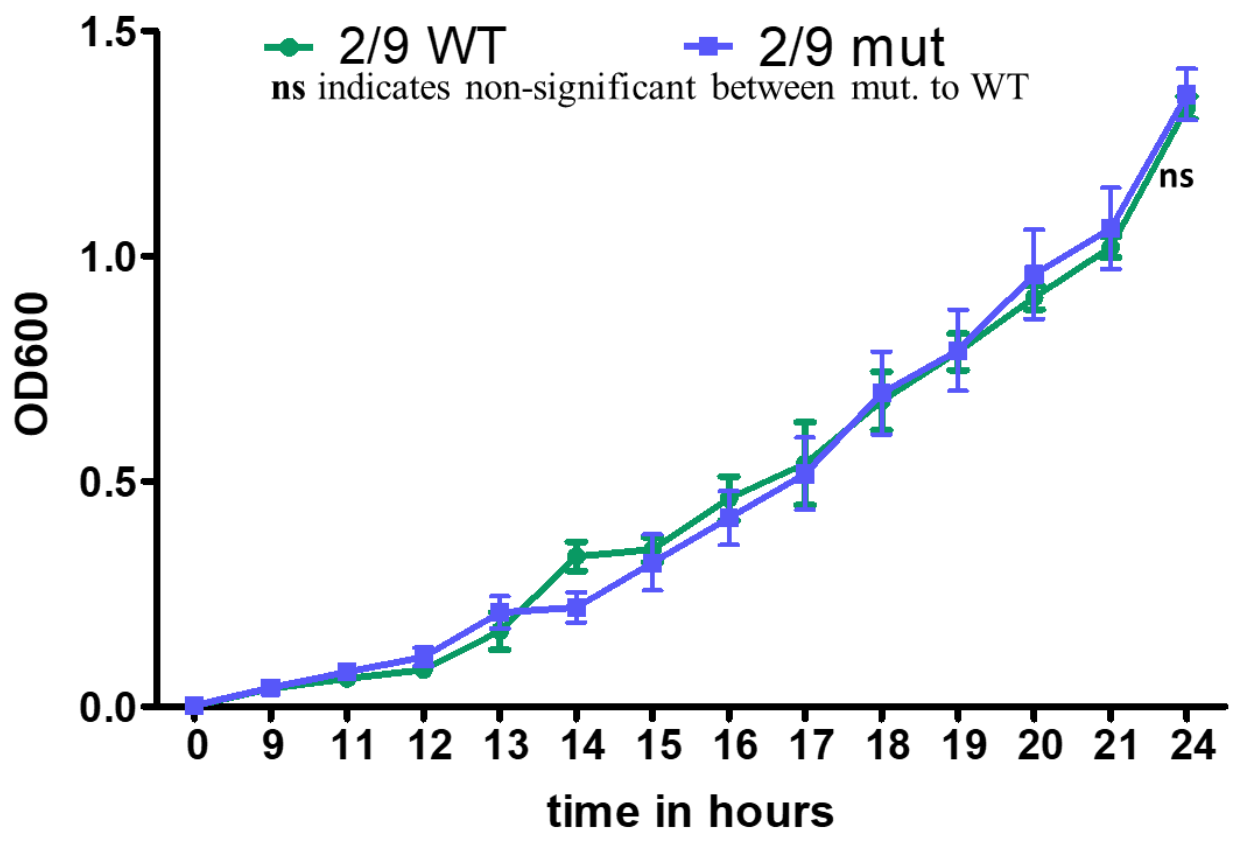


(C) $1 / 2$ in $\mathrm{NO}_{3}^{-}$

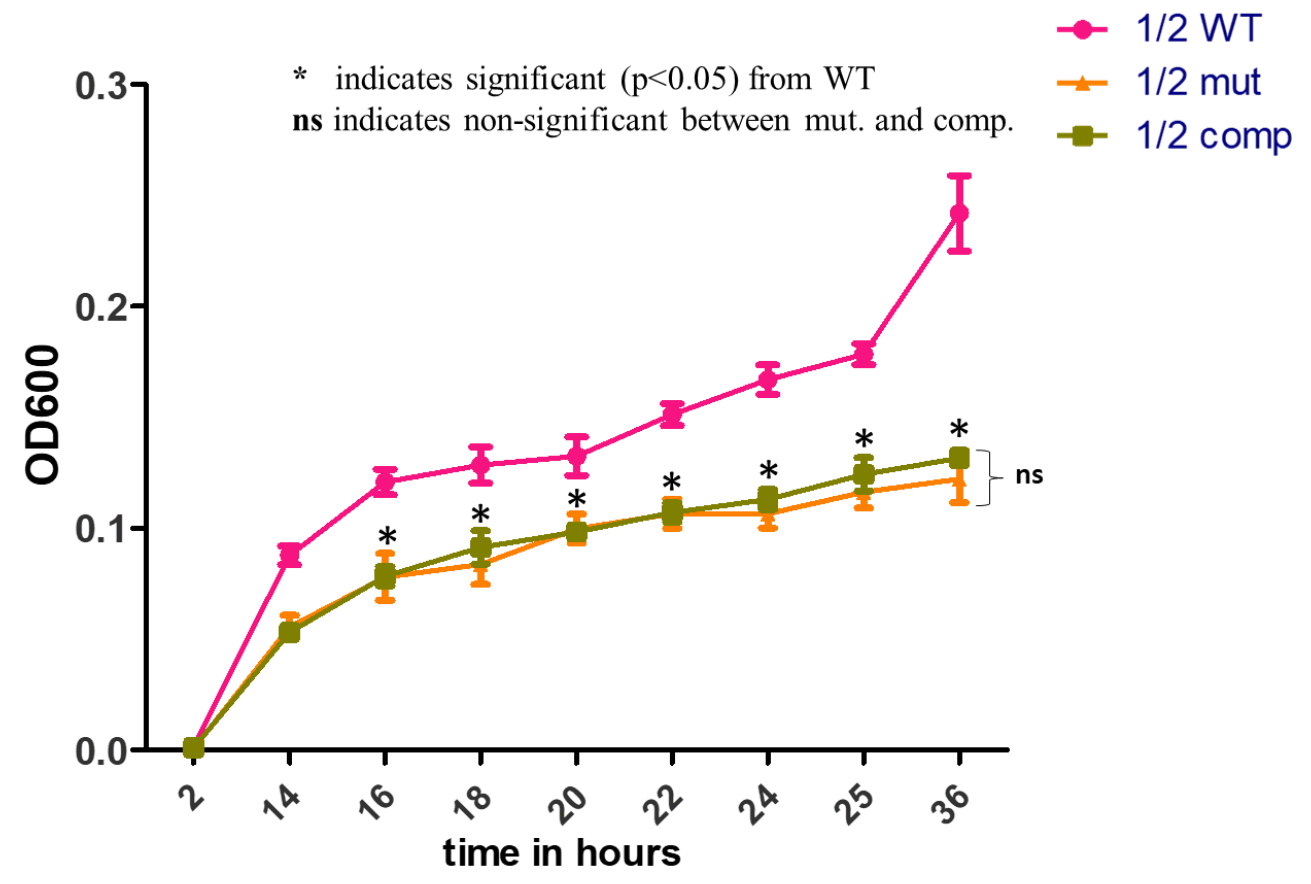

(D) $2 / 9$ in $\mathrm{NO}_{3}^{-}$

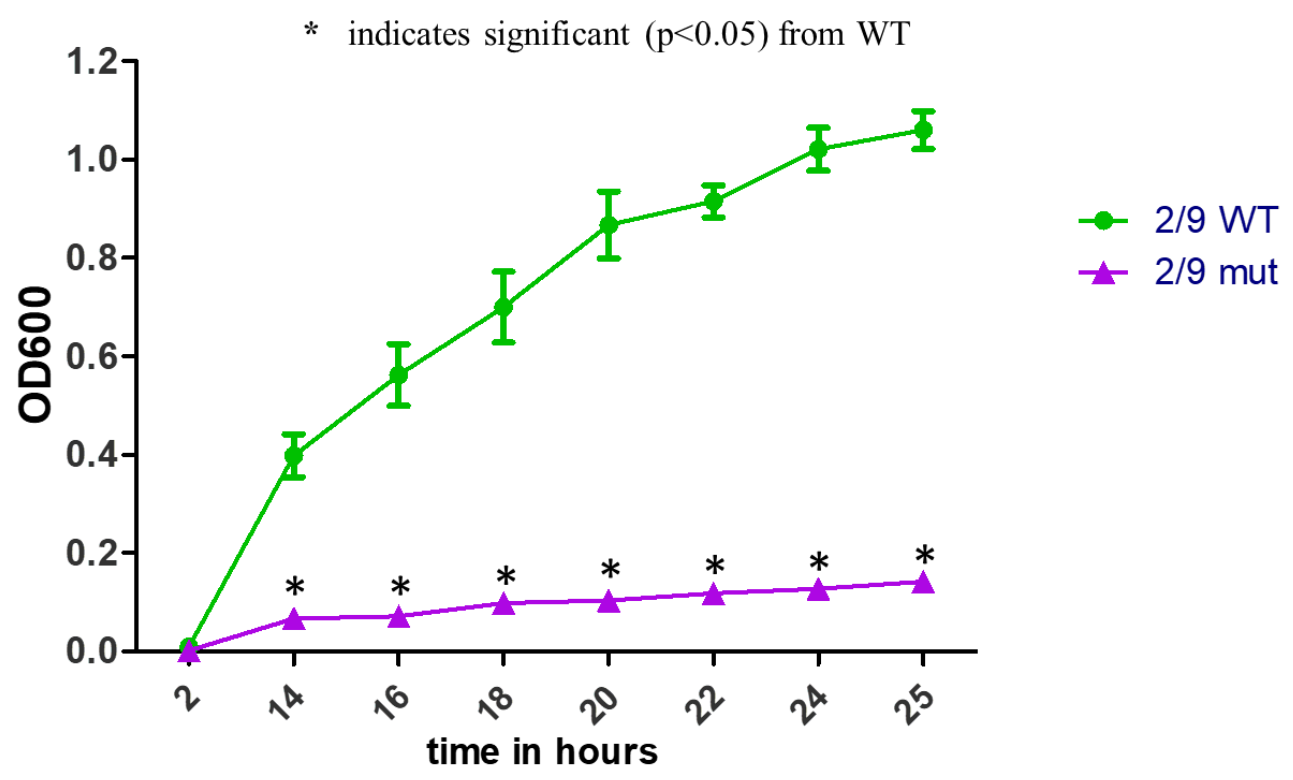

Figure 2. 6 Growth curves for um03848 mutants.

The haploid $U$. maydis strains $(\mathbf{A}, \mathbf{C}) 1 / 2$ and $(\mathbf{B}, \mathbf{D}) 2 / 9$, were grown in YEPS medium overnight at $28{ }^{\circ} \mathrm{C}$, collected by centrifugation, washed with sterile water and resuspended in $50 \mathrm{ml}(\mathbf{A}, \mathbf{B})$ YEPS medium or $(\mathbf{C}, \mathbf{D})$ medium with nitrate as a source. After cells were grown at $28{ }^{\circ} \mathrm{C}$, on a rotary shaker at $250 \mathrm{rpm}$ and $\mathrm{OD}$ at $600 \mathrm{~nm}$ was taken at time indicated in $\mathrm{X}$-axis. Each point on the graph is an average of three 
biological replicates, standard error bars are indicated. For each time point, two-way ANOVA was performed followed by Bonferroni posttests to compare the growth between different strains. Asterisk $(*)$ indicates the significant difference $(p<0.05)$ between the wild type and mutant strains.

\section{Growth of um03848 mutant in different stress media was not affected. YEPS}

supplemented with $1 \mathrm{M}$ sorbitol or $1 \mathrm{M} \mathrm{NaCl}$ as high osmotic media as well as YEPS supplemented with $1 \mathrm{M}$ Congo red (CR) as cellular stress medium were used as the stressors. All mutants and wild types were spotted as serial 10-fold dilutions onto these media. There was no observable difference in the growth of um03848 deletion mutants in any of the stress media (Figure 2.7 A-C).

\section{(A)}
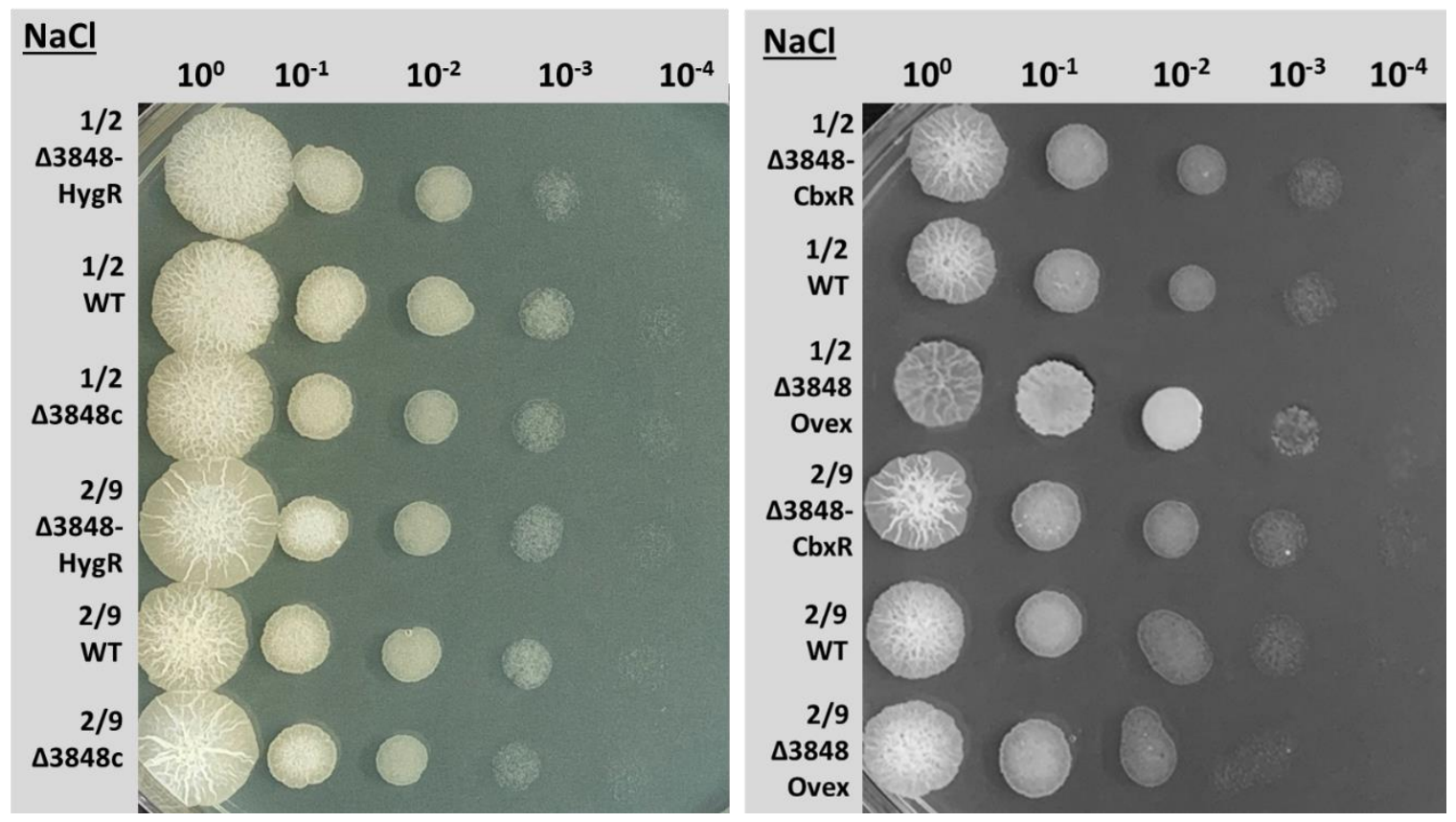
(B)

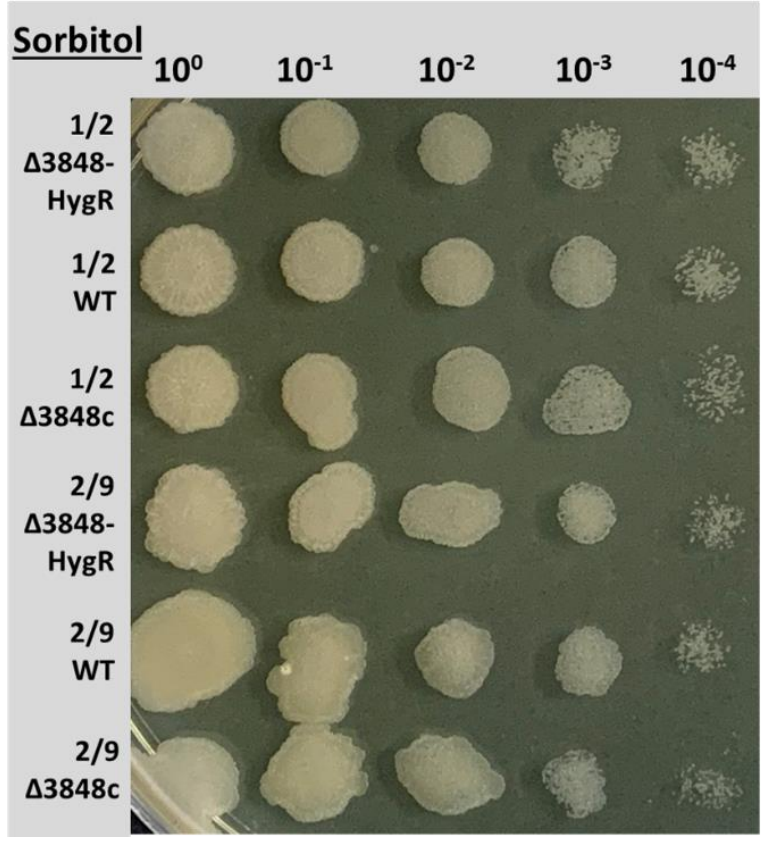

(C)

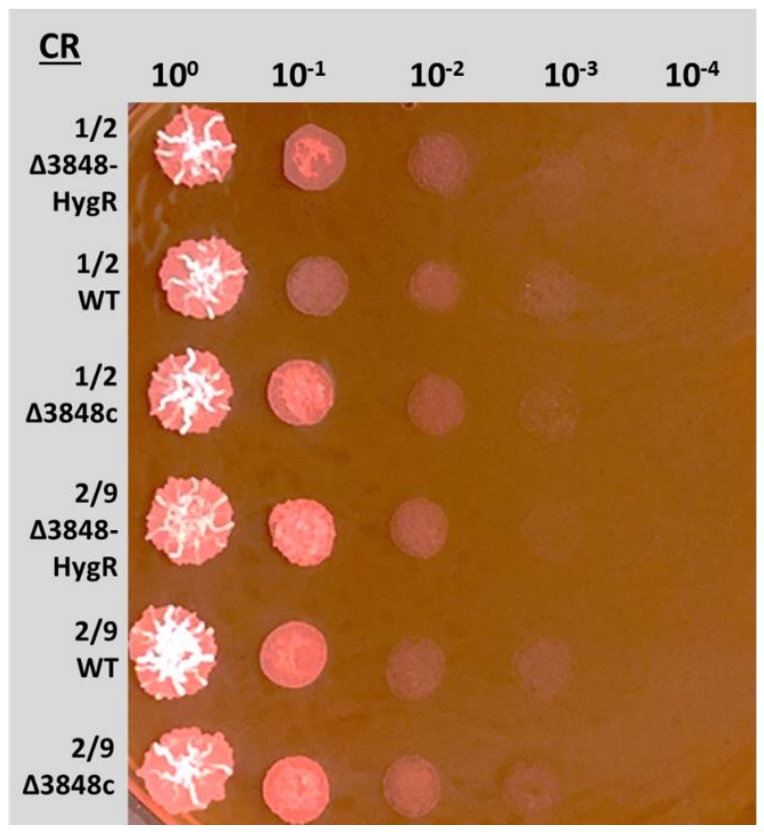

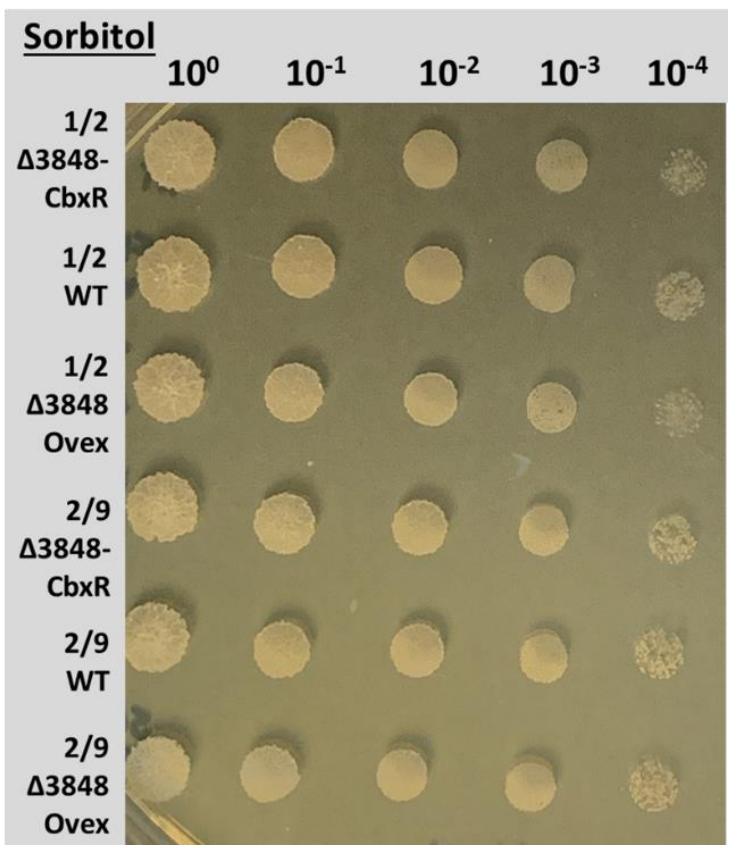

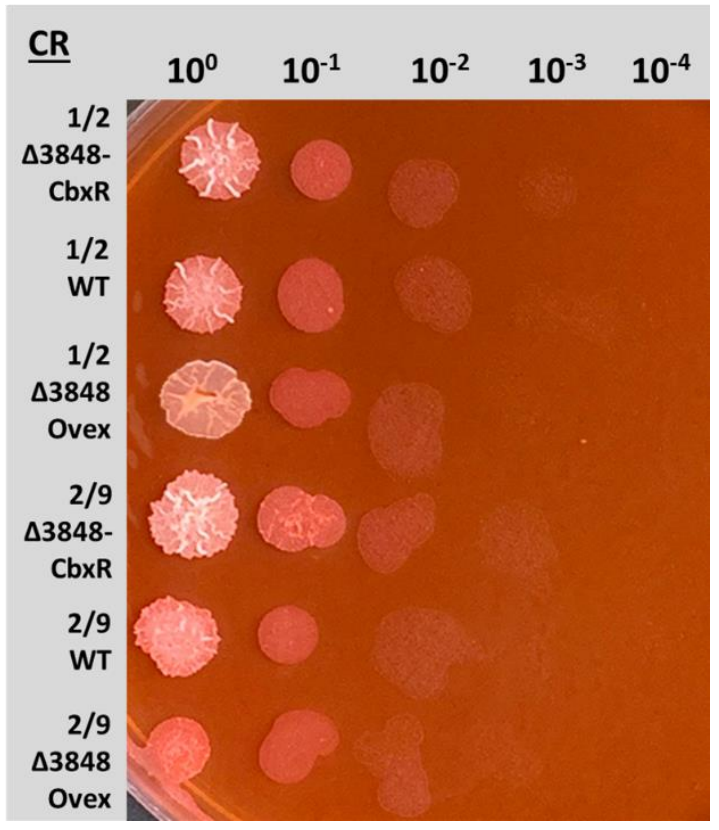

Figure 2. 7 Growth of mutants on stress media.

The 10 -fold serial dilutions of wild types, $\Delta u m 03848$, um03848c and $u m 03848^{\mathrm{Otef}}$ strains were spotted onto YEPS supplemented with (A) 1 M Sodium Chloride ( $\mathrm{NaCl})$, or (B) 1 M Sorbitol or (C) $1 \mathrm{mM}$ Congo red (CR). $\mathrm{NaCl}$ and Sorbitol are osmotic stressors and Congo red is a cell wall stressor. 


\section{Deletion of um03848 affects the degree of pathogenesis, in a background-specific}

manner. The compatible opposite mating strains were pre-mixed and injected into maize seedlings to determine their ability to infect the host plant. For the solopathogenic SG200, and diploid strain D132, the wild type and mutant strain were each injected without a partner. The percentage of plants showing specific virulence symptoms are presented in a graph (Figure $2.8 \mathrm{~A}$ ), which indicates that the $\Delta u m 03848$ significantly reduced the virulence of the infection to the plant as compared to the wild type $(1 / 2$ albl $X 2 / 9 a 2 b 2)$. The complemented $\Delta u m 03848 \mathrm{c}$ mating partners were able to rescue the phenotype of $\Delta u m 03848$ (Figure 2.8). The overexpression of um03848 showed similar degree of pathogenicity as the wild type strains (Figure 2.8).

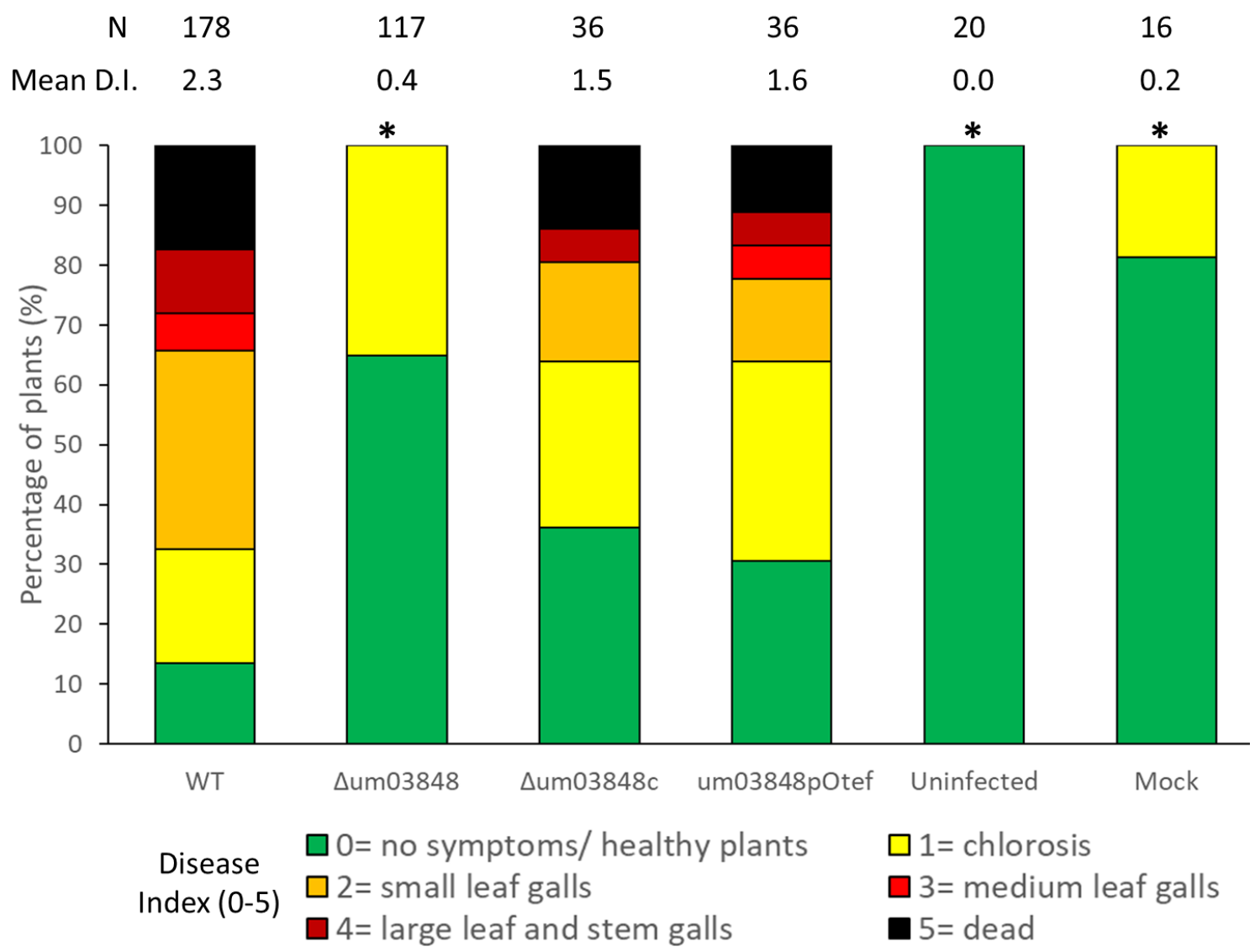

Figure 2. 8 Pathogenicity assay with indicated strains of um03848 mutants. 
The indicated strains of $U$. maydis were grown to exponential phase, washed twice with sterile distilled water, concentrated to $\mathrm{OD}_{600}=3$, pre-mixed with their compatible partner and injected into 7-day old maize (Zea mays) seedlings (Early Golden Bantam). Disease symptoms were scored 14 days post infection (dpi). The graph displays the percentage of plants with specific symptoms of infection. The $\mathrm{X}$ axis indicates the paired $(1 / 2 \times 2 / 9)$ background injected into maize plant. The uninfected group indicates the plants that were grown without injecting anything. The Mock group indicates the plants that were injected with sterile distilled water. The disease symptoms were categorized from 0 to 5 as depicted at the bottom of the figure. Numbers on the top $(\mathrm{N})$ represent the number of plants infected and mean for disease indices (DI). The data was analyzed using KruskalWallis test followed by post-hoc comparison and an asterisk $(*)$ indicates significant difference $(\mathrm{p}<0.05)$ compared to those where both partners were wild type strains $(1 / 2 X$ 2/9). The significant reduction ( $\mathrm{p}<0.05$ ) of plant pathogenesis was seen in $\triangle u m 03848$ strain. This reduction was fully recovered by the ectopic complementation of um03848 in $\Delta u m 03848$ (um03848c). The overexpression of um03848 (um03848 ${ }^{\text {Otef }}$ ) showed similar virulence as that of wild type strains.

In a separate experiment, plants were injected with um03848 deleted in the solopathogenic SG200 background. Plants were also injected with a diploid D132 strain where one copy of um03848 was deleted. Both groups showed similar severity of infection as their respective wild type progenitors (Figure 2.9). This indicated that the reduced pathogenicity is due to factors post-mating and is therefore not under control of $a$ and $b$ loci genes directly. 


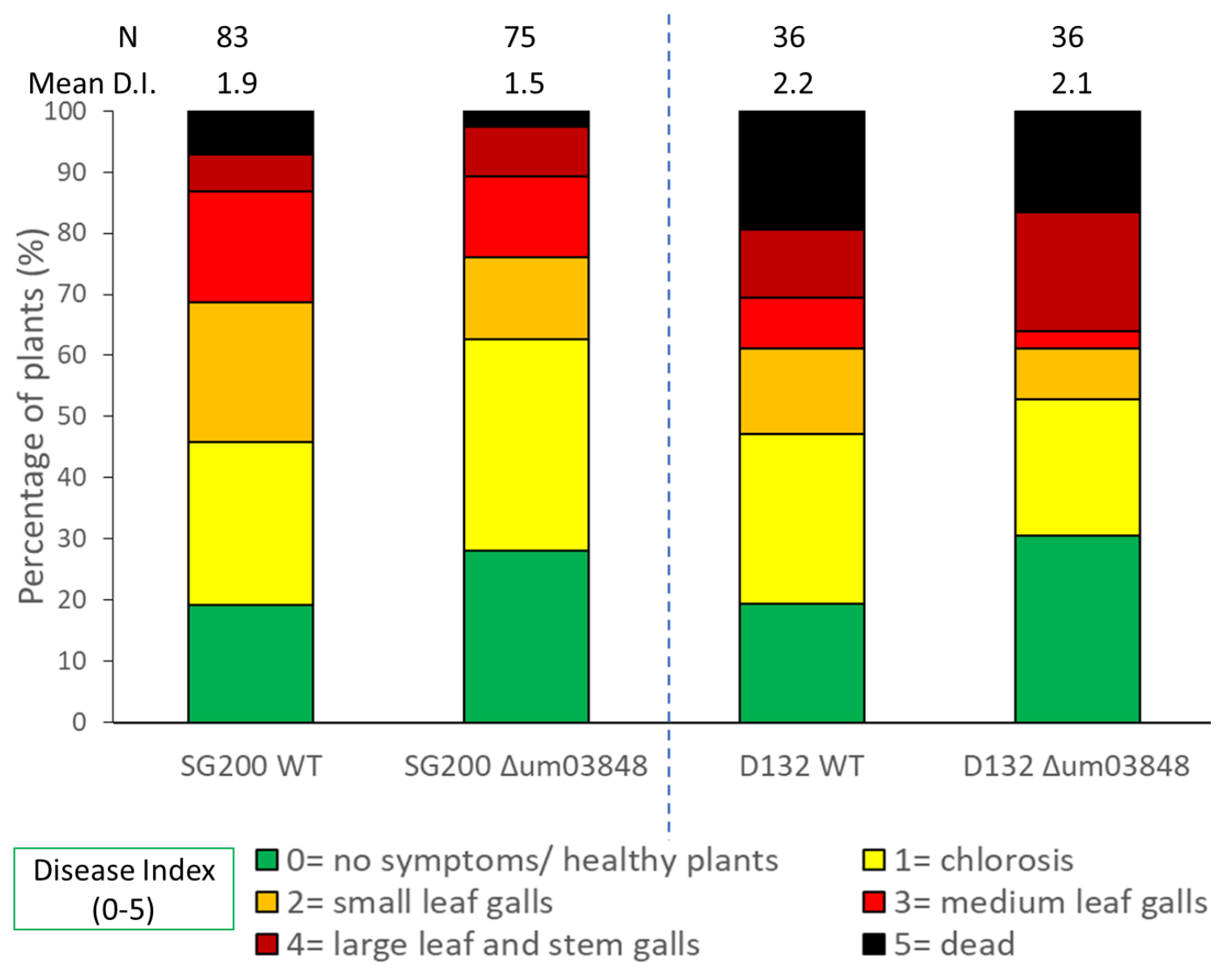

Figure 2.9 Plant infection using solopathogenic strain SG200 and diploid D132. The indicated strains were injected into 7day old maize seedlings. Disease symptoms were scored 14 days post infection (dpi). The disease symptom was categorized from 0 to 5 as depicted at the bottom. The Y-axis represents the percentage of plants with specific symptoms of infection. The $\mathrm{X}$-axis indicates the background that was injected into maize plant. The data were analyzed using Kruskal-Wallis test followed by post-hoc comparison and an asterisk $(*)$ indicates significant difference $(\mathrm{p}<0.05)$ compared to their respective wild type strains: SG200 WT or D132 WT. Despite lower mean D.I. indicates reduced virulence, the analysis did not find a statistically significant difference between SG200 $\Delta u m 03848$ and its WT progenitor. Similarly, the virulence levels of D132 $\Delta u m 03848$ and D132 WT were not statistically significant.

Furthermore, we analyzed whether or not functional copies of the um03848 gene in both haploid partners were required for full pathogenicity. The $\Delta u m 03848$ was combined with the wild type strain of opposite mating-type and compared with the infections due to either both wild type or both mutant pairings (Figure 2.10 A, B). The 
1/2 $\Delta u m 03848 \mathrm{X} 2 / 9 \mathrm{WT}$ showed significantly reduced virulence, in a manner similar to that of $\Delta u m 03848(1 / 2 X 2 / 9)$. However, $1 / 2 \mathrm{WT}$ X 2/9 $\Delta u m 03848$ only displayed a slight reduction in Disease Index that was not significant compared to WT infections. This indicted that the um03848 affects the pathogenicity to the plant, dependent on the specific genetic background of the strain (Figure 2.10 A). Similar results were observed when the infection assay was performed in independently constructed $\Delta u m 03848:: C b x R$ mutant (Figure 2.10 B).

(A)

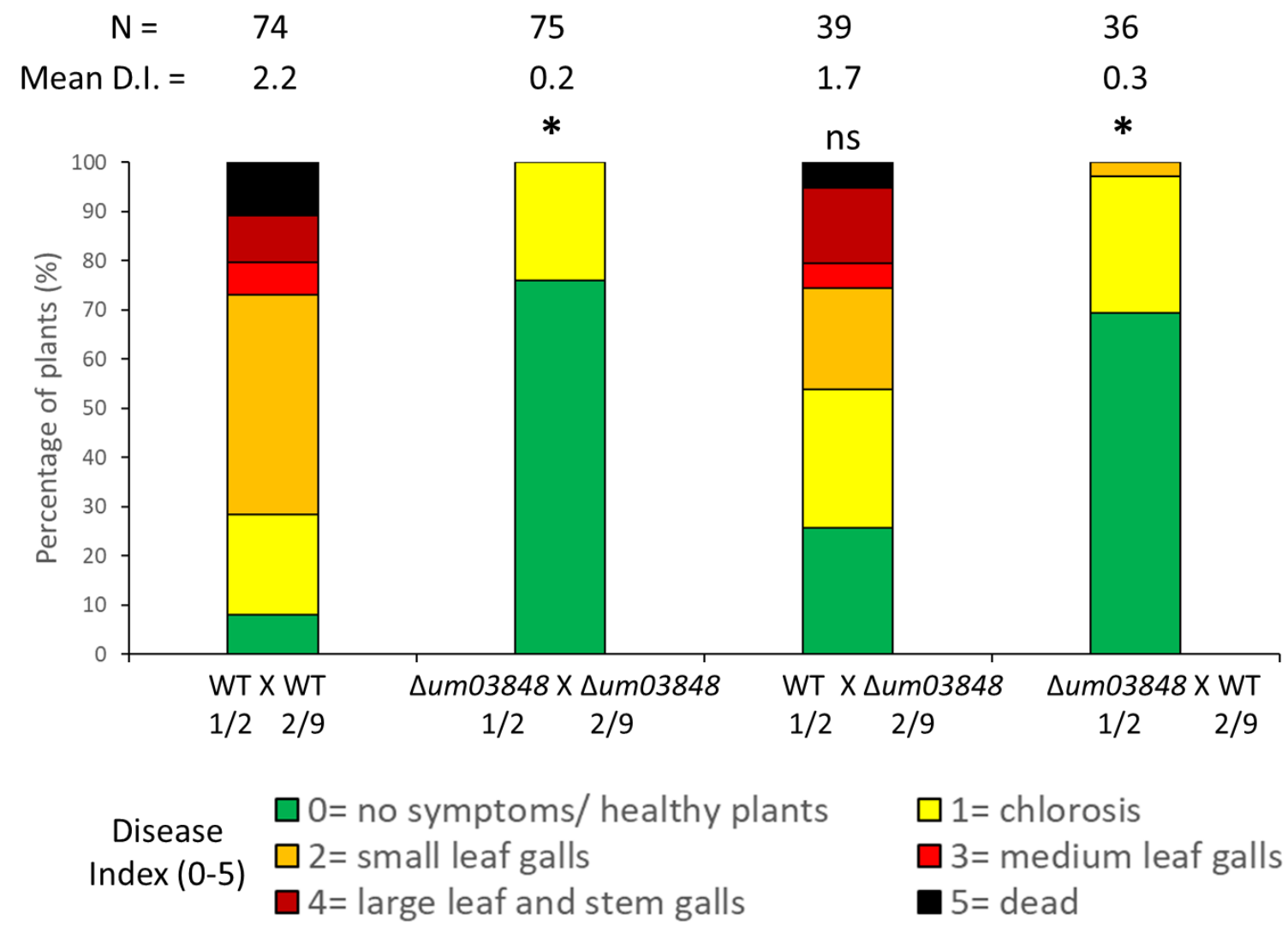


(B) um03848::cbx ${ }^{R}$

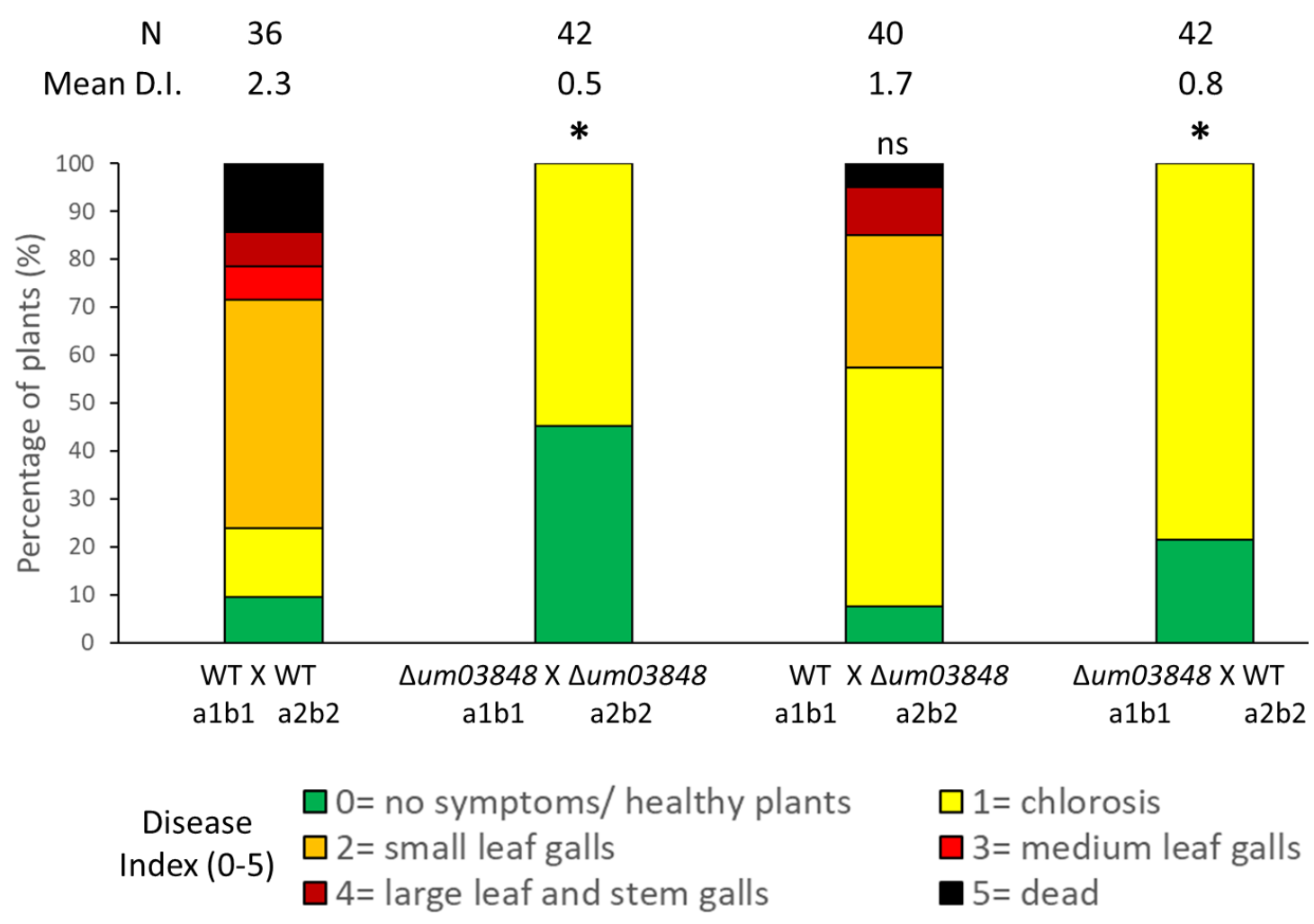

Figure 2.10 Are functional copies of the um03848 gene in both partners required for pathogenicity?

The $\Delta u m 03848$ strains were combined with the wild type strain of opposite mating-type and compared with infections due to either both wild type or both mutant pairings. The graphs display the percentage of plants with specific symptoms of infection. The data were analyzed using Kruskal-Wallis test followed by post-hoc comparison and an asterisk $(*)$ indicates significant difference $(\mathrm{p}<0.05)$ compared to $1 / 2$ WT X 2/9 WT. The combination $1 / 2 \Delta u m 03848 \times 2 / 9 W T$ was only slightly more virulent when compared to $\Delta u m 03848(1 / 2 \times 2 / 9)$ but was significantly reduced when compared to the $1 / 2$ WT X 2/9 $W T$. Similar results were observed in the independently constructed $u m 03848:: h y g^{R}$ and um03848:: $c b x^{R}$ mutants. (A) the deletion um03848 mutant was generated by homologous recombination of um03848 in U. maydis by hygromycin resistant (HygR) cassette. (B) the deletion um03848 mutant was generated by homologous recombination of um03848 in $U$. maydis by carboxin resistant $(C b x R)$ gene.

Additional measures of virulence. The effect of the deletion of um03848 on the virulence of the fungus on the host plant was measured by two different plant growth parameters. These parameters were the height of plant and total number of leaves. The 
plants infected with the wild type cross (1/2 X 2/9) strains were compared with those infected with um03848 deletion ( $\Delta u m 03848)$, complementation (um03848c) and overexpression (um03848 ${ }^{\text {Otef }}$ ) cross strains. The total number of leaves and length of the plants were measured on the day of infection and 8 dpi. Measurements were not taken after 8 days, as many plants were drooping and some were dying due to infection, thereby making accurate length measurements difficult. The difference in the measurements of leaves and plant height from 8 dpi compared to the day of infection were plotted (Figure $2.11 \mathrm{~A}, \mathrm{~B})$. The plants infected with $\Delta u m 03848$ grew significantly taller, indicating reduced infection, than those infected by wild type strains (Figure 2.11 A). The average number of leaves produced was significantly higher in plants infected with $\Delta u m 03848$ compared to plants infected with wild type (Figure 2.11 B). However, the differences observed in the length of plant (Figure 2.11 A) and number of leaves (Figure 2.11 B) in the plants infected by complementation (um03848c) and overexpression (um03848 $\left.{ }^{\mathrm{Otef}}\right)$ strains were closer to those infected by wild type strains. These results indicate that deletion of $u m 03848$ results in the significant reduction in virulence of the fungus, but the overexpression also did not cause additional harm to the host plant. 

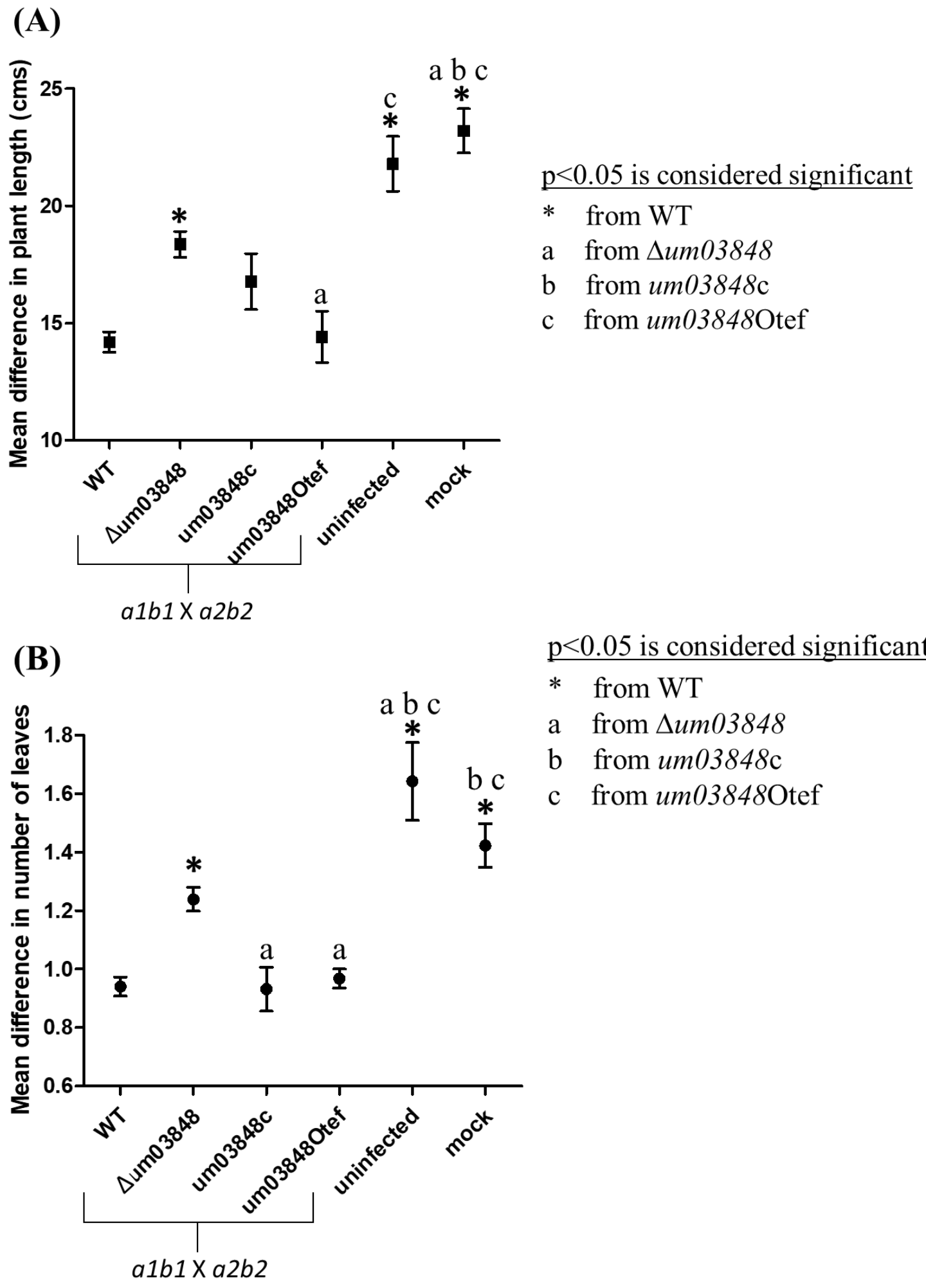

Figure 2. 11 Plant growth parameters.

Effect of um03848 deletion, complementation and overexpression strains on (A) plant height $(\mathrm{cm})$ and (B) leaf count. The plants were infected with wild type, WT, $(1 / 2$ albl $X$ 2/9 a2b2), $\Delta u m 03848$ (a1b1 X a2b2), um03848c (alb1 X a2b2), um03848 Otef (a1b1 X 
$a 2 b 2$ ) and sterile distilled water (Mock). Some plants were grown normally without infecting with anything (uninfected) as an additional control. The length and the number of leaves produces on 0-dpi (day post infection) and 8-dpi were measured. The mean of the differences ( $8 \mathrm{dpi}-0 \mathrm{dpi}$ ) in plant height and leaf count is presented in figure. Statistical analysis was done by one-way ANOVA in GraphPad prism, followed by Tukey's test to compare in between groups. $\mathrm{P}$ value $<0.05$ was considered significant and represented by different symbols in graph, stated in legend. The plants infected with $\Delta u m 03848$ showed significant increase in the plant height and the number of leaves when compared to those infected by wild type.

\section{Gene expression of ump2 and mating gene targets for $\Delta u m 03848$ grown under low}

ammonium conditions. The background specific effects of um03848 deleted mutants on the virulence to the host plant led us to evaluate the expression of mating target genes that could affect virulence. For this, qRT-PCR was performed for $\Delta u m 03848$ and wild type strains grown on high and low ammonium medium to examine the expression of genes involved in the mating pathway. These genes include: $m f a$, encoding pheromone precursor; $p r f 1$, encoding a transcription factor; $b$ genes $(b E$ and $b W)$, whose heterodimerization is required for mating and progression of the pathogenic program; and rbfl, encoding a transcription factor required for pathogenesis. The expression of the high-affinity ammonium transceptor, ump2, was also studied. The expression of all the strains were normalized against the endogenous control gene, eif 2 , and then compared to their respective strains grown in high ammonium, e.g., 1/2 WT low was compared with 1/2 WT high and $1 / 2 \Delta u m 03848$ low was compared with $1 / 2 \Delta u m 03848$ high. The WT as well as the $\Delta u m 03848$ strains grown under low ammonium showed upregulation of the ump2 gene. However, when the mutant grown on low ammonium was compared with the WT grown on same condition, the ump2 expression was significantly downregulated in the mutants (Figure 2.12 A). The mating target genes were expressed differently in $1 / 2$ $\Delta u m 03848$ and 2/9 $\Delta u m 03848$ (Figure 2.12 B and D). The most prominent differences 
were in the expression of $b$ genes and transcription factors, Prf1 and Rbf1. We believe that due to significant downregulation of $b W 1$ and $r b f 1$ genes, the $1 / 2 \Delta u m 03848$ mutant is less virulent than 2/9 $\Delta u m 03848$. The $b W 1$ and $r b f 1$ genes were upregulated (lower level than WT) in $1 / 2 \Delta u m 03848 \mathrm{c}$ strain (Figure $2.12 \mathrm{~B}$ ), which might be reason behind rescued pathogenicity of $\Delta u m 03848 \mathrm{c}$. The expression of $u m 03848$ in $1 / 2 \Delta u m 03848 \mathrm{c}$ was significantly upregulated on low ammonium. However, this expression was slightly below $(\mathrm{p}$-value $=0.1)$ expression of $u m 03848$ in $1 / 2 \mathrm{WT}$ grown on low ammonium (Figure $2.12 \mathrm{C}$ ). This might be the reason why expressions of $b W 1$ and $r b f 1$ in $1 / 2$ $\Delta u m 03848 \mathrm{c}$ was not fully recovered.

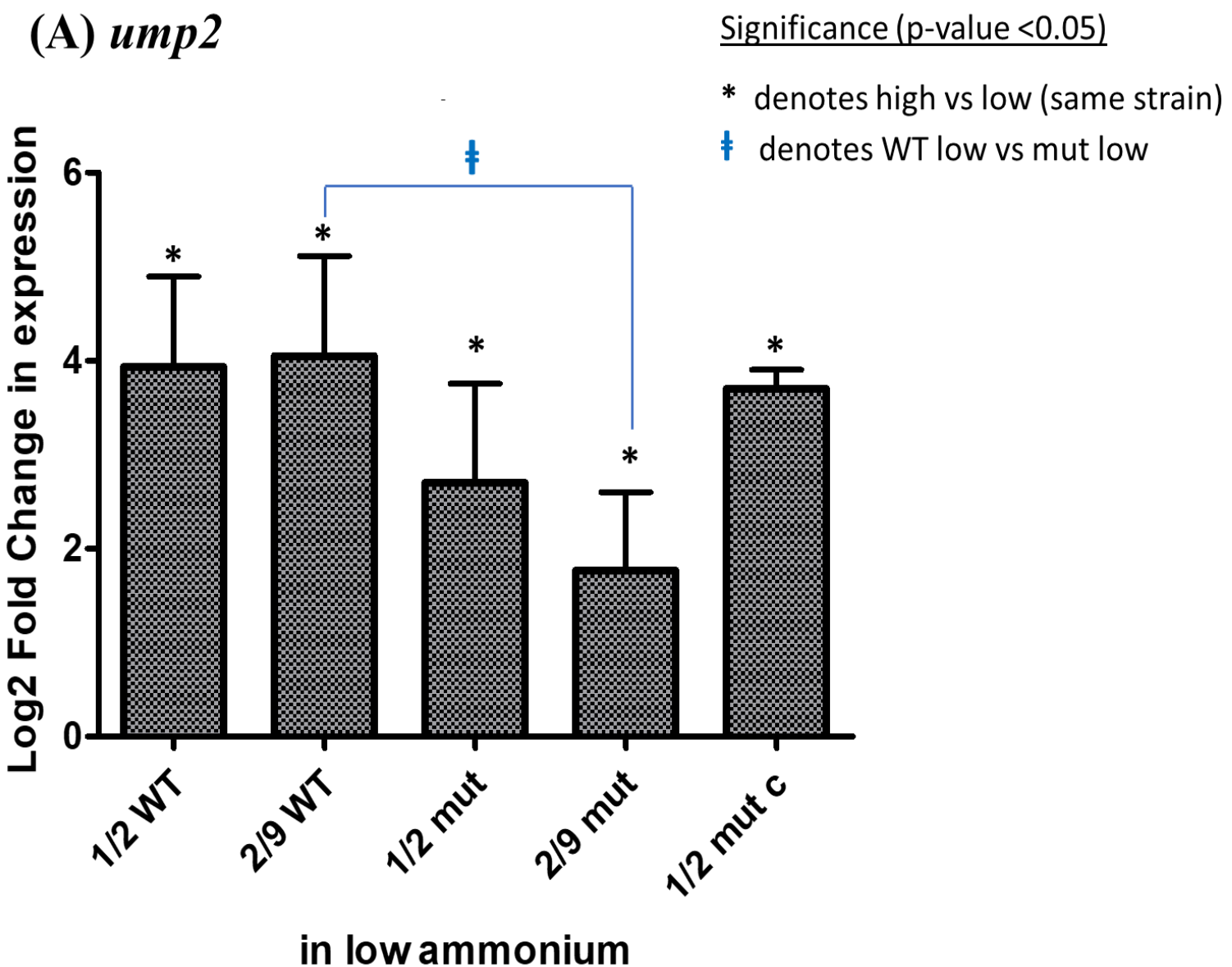




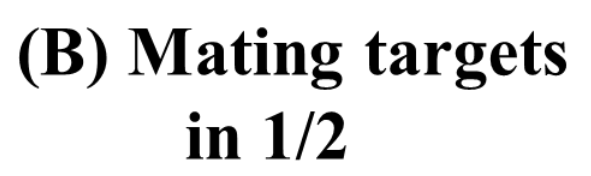

Significance ( $p$-value $<0.05$ )

* denotes high vs low (same strain)

\# denotes WT low vs mut low

d denotes mut low vs comp low

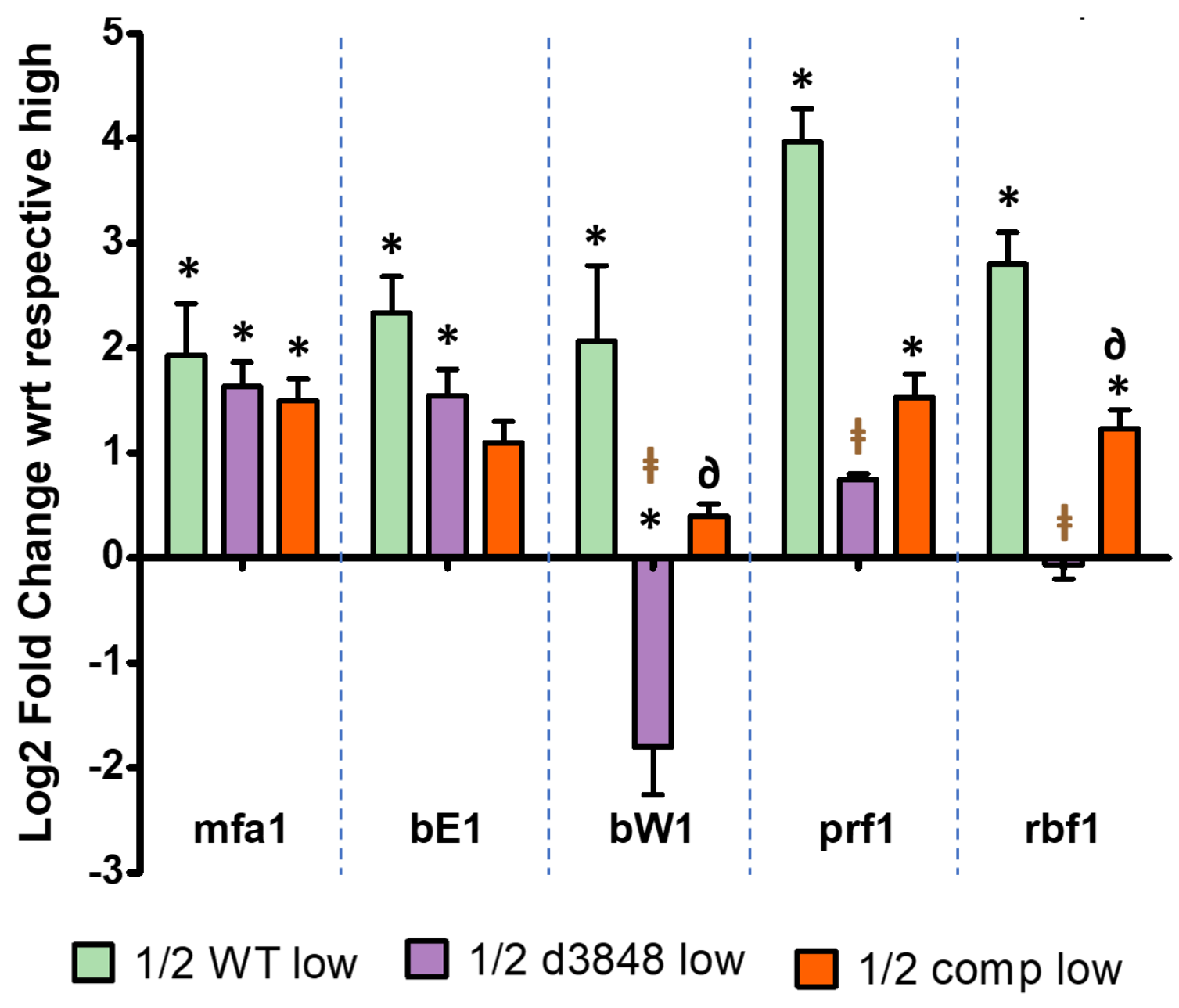


(C) $u m 03848$

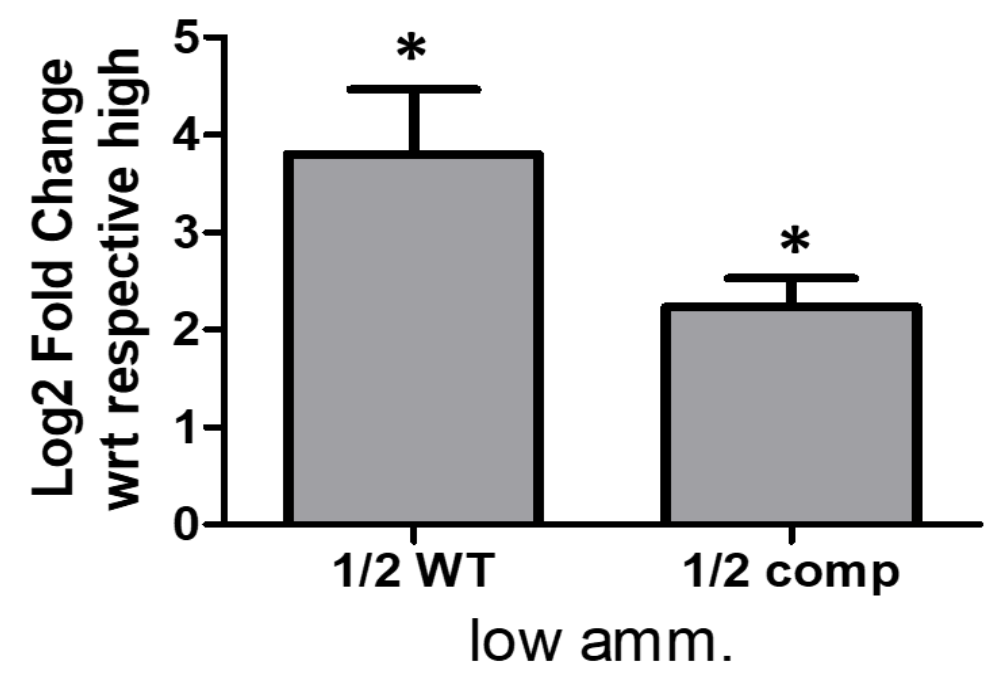

(D) Mating targets in $2 / 9$
Significance ( $p$-value $<0.05)$

* denotes high vs low (same strain)

\# denotes WT low vs mut low

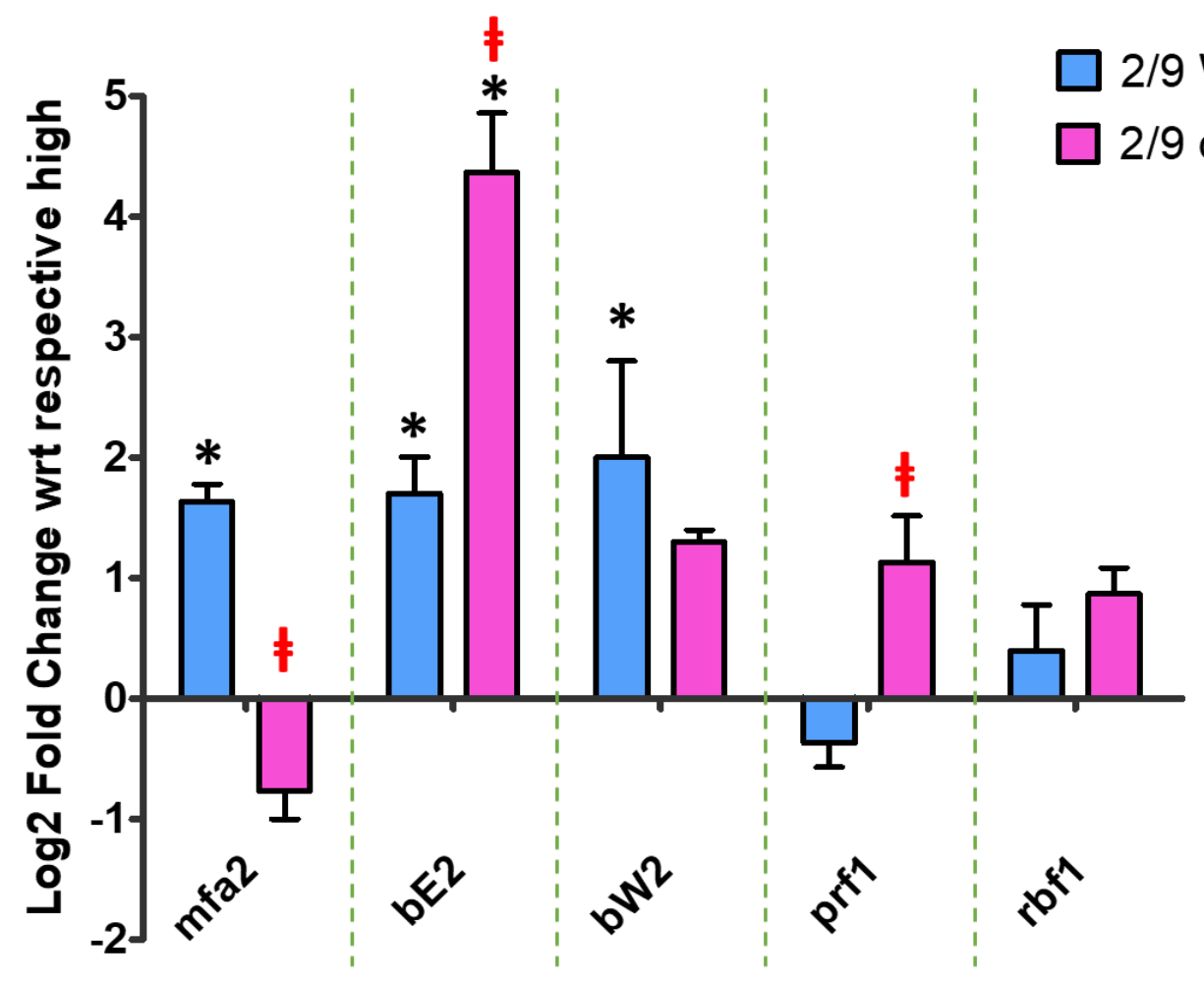

Figure 2. 12 The average log2 fold changes in different gene expression by um03848 mutants grown under low ammonium condition. 
The wild type and $\Delta u m 03848$ strains in $1 / 2$ and $2 / 9$ backgrounds were grown under low ammonium and high ammonium conditions. The complementation mutant, 1/2

$\triangle u m 03848 \mathrm{c}$ was also grown in the same conditions. The qRT-PCR was performed on the total RNA extracted from these strains for the (A) high affinity ammonium transceptor, ump2 (B) genes in mating pathway for $1 / 2$ background, (C) um03848 gene in WT vs $1 / 2$ $\Delta u m 03848 \mathrm{c}$ under low ammonium conditions and (D) genes in mating pathway for $2 / 9$ background. The log2 fold changes in expression of the strains grown under low ammonium were normalized against eif 2 and are expressed with respect to (wrt) the same strain grown under high ammonium. Bars represent the averages of biological triplicates and standard errors are indicated in the graphs. The positive values represent increased expression whereas negative values reflect the decreased expression relative to high ammonium conditions. One-way ANOVA followed by Tukey's Multiple Comparison Test was performed in GraphPad Prism. P-value $<0.05$ is considered significant. * indicates the significant difference in the expression level of target gene for the strain grown on low ammonium with respect to same strain grown on high ammonium. $\neq$ indicates the significant difference in the expression level of the mutant on low when compared with wild type on low. $\partial$ denotes significance between the mutant on low ammonium compared to the complemented strain on low ammonium.

\section{Discussion}

In the basidiomycete fungus, $U$. maydis, the environmental signals induce and coordinate the discrete dimorphic transition during sexual development. In this fungus, mating of two compatible haploid sporidia is a prerequisite for the dimorphic switch and subsequent pathogenic development in the host plant ${ }^{126}$. Nutrient limitation, specifically for nitrogen, is one of the factors responsible for the transition. Ammonium and glutamine are the preferred sources of nitrogen for fungi. Nitrate and nitrite are considered unfavorable nitrogen sources because the reduction of nitrate/nitrite into ammonium is an energy-expensive process, ${ }^{31,100}$. Therefore in nitrate assimilation, the synthesis of the metabolic enzymes involved only occurs if there is nitrogen starvation for the preferred nitrogen sources and the presence of nitrate ${ }^{53}$.

Nitrogen Metabolic Repression (NMR) is a regulatory strategy in microorganisms that restricts the utilization of unfavored nitrogen sources in the presence of favored 
nitrogen sources. Nit2 is the major positive regulator of NMR in many filamentous fungi ${ }^{3,21}$ including $U$. maydis ${ }^{6}$. In $U$. maydis, the genes encoding nitrate transporter $(n r t)$, nitrate reductase (narl) and putative nitrite reductase (nirl) are present in one gene cluster $^{55}$. The nitrate reductase (narl) expression is induced at the transcriptional level in the absence of favored nitrogen and presence of nitrate in filamentous fungi by Nit2/AreA ${ }^{3}$. In U. maydis, there is transcriptional regulation of narl ${ }^{56}$ in which the promoter of narl is tightly regulated by the presence of nitrate ${ }^{127}$. In the transcriptional analysis of $U$. maydis grown in different nitrogen media, there was significantly higher transcript accumulation of narl and nrt for cells grown in the medium with nitrate. However, in $\Delta$ nit 2 , the transcript accumulation was much lower than wildtype ${ }^{6}$. This indicates that the expression of genes in nitrate metabolism is highly controlled, which is partially dependent on the NMR master regulator gene, nit2.

We demonstrated that $u m 03848$, putative nitrite reductase (nirl) gene in $U$. maydis has high similarity with the known nitrite reductase genes in other filamentous fungi (Table 2.4, Figure 2.1 A). In the conserved domain analysis, we observed that the predicted UM03848 protein consists of Rieske-like, Ferredoxin and [NAD(P)H]-Nir large subunit domains (Figure 2.1 B). From qRT-PCR, we observed that the expression of the U. maydis nitrite reductase gene, um03848, was significantly upregulated when cells were grown in a low ammonium (nitrogen starvation) condition relative to that when cells were grown in high ammonium condition (Figure 2.2). These results led us to explore the functional properties of gene um03848, especially in terms of mating and pathogenicity in $U$. maydis. 
The high affinity ammonium transporters of filamentous fungi are involved in mycelial proliferation and may also act as ammonium sensors which stimulate hyphal proliferation in response to low levels of ammonium enrichment ${ }^{128}$. The deletion of the high affinity ammonium transceptor, ump2, results in the loss of filamentation when grown on low ammonium medium. However, the overexpression of ump2 resulted in the higher filamentation on low ammonium ${ }^{82}$. Our study showed that the deletion of nitrite reductase gene, um03848, does not affect the sensing or response of $U$. maydis towards ammonium, as deleted mutants produced haploid filaments like their respective WT strains grown on low ammonium medium (Figure 2.4). Similarly, the overexpression of um03848, also didn't have any effect on the filamentation of the fungus on low ammonium. However, deleted mutants in different fungal backgrounds showed significant reduction in ump2 gene expression (Figure $2.12 \mathrm{~A}$ ).

The ability of haploid $\Delta u m 03848$ strains to grow in medium with different nitrogen sources was examined and compared to the growth of their respective wild types. On solid medium with nitrate as a sole source of nitrogen, the growth of the $\Delta u m 03848$ mutants was reduced compared to the wild type (Figure 2.5) suggesting inability of the fungus to reduce nitrite; thus, $\Delta u m 03848$, affects the growth of fungus in nitrate. This was confirmed by the study of time-course growth of $\Delta u m 03848$ in liquid medium (Figure 2.6 C, D). However, 2/9 WT unexpectedly had higher growth on nitrate medium on plate as well as in liquid culture, which might be a unique phenotype of 2/9 background that wasn't studied before. The null mutant of the NiR gene, YNI1, in $H$. polymorpha, excreted nitrite when cells were incubated in nitrate ${ }^{129}$. In yeast $H$. anomala, the presence of nitrite caused inactivation of nitrate reductase activity ${ }^{130}$. 
Nitrite has been described to have toxic effects in many fungi, inhibiting their growth ${ }^{131-}$

133. Thus, inability of $\Delta u m 03848$ to grow in nitrate medium could be to maintain the nitrite levels below lethal concentrations inside the cell. However, there was no difference in growth of $\Delta u m 03848$ strains on other nitrogen source media (Figure 2.5). Similarly, when mutants were grown in different stress media such as Congo Red, Sorbitol and $\mathrm{NaCl}$, there was no difference in growth from wild types grown in the same media (Figure 2.7).

When plants were infected with $\Delta u m 03848$ strains, there was a drastic reduction in virulence to the plants (Figure 2.8), and this reduction was reversed in the complemented strains. On the other hand, the overexpression of um03848 had no apparent effect on virulence beyond that of WT. Similarly, deletion of um03848 in the solopathogenic haploid SG200 strain and deletion of one copy deletion of um03848 in the diploid D132 strain had no obvious effects on pathogenicity to plants (Figure 2.9). This indicates that the role of $u m 03848$ on virulence is a post-mating phenomenon and that only a single copy of the gene is sufficient after cell fusion (or its equivalent) for full function in plant infection. This result is similar to the reduced plant pathogenicity seen in F. oxysporum f. sp. melanis mutant in which a nitrite reductase gene was disrupted ${ }^{125}$. In host-pathogen interactions, efficient pathogen nutrition and the ability of filamentous fungi to acquire nutrition from host is the prerequisite for successful colonization and fungal fitness ${ }^{134}$. A biotrophic pathogen such as $U$. maydis, has total dependency on extracting nutrients from living host cells to complete its life cycle ${ }^{135}$. Nitrate is one of the major components in soil as well as in the fertilizer used in croplands ${ }^{136}$. The nirl mutant in Colletotrichum acutatum displayed defects in appressorium development ${ }^{137}$. 
The Nir1 protein is an ortholog of NirA in A. nidulans, which is a pathway-specific activator of nitrate utilization genes or NMR. Similarly, deletion of Nit2 in U. maydis delayed the initiation of formation of dikaryotic filaments at the site of infection and significantly reduced virulence ${ }^{6}$. Therefore, the ability of fungus to take up and utilize nitrate is important for the proliferation of the fungus inside host tissue. Hence, the deletion um03848, gene encoding nitrite reductase- a second step in reduction of nitrate reduction to ammonia, reduces the ability of fungus in pathogenicity to the host (Figure 2.8).

To study if functional copies of $u m 03848$ in both opposite partners is required for pathogenicity, we mixed $\Delta u m 03848$ with its opposite wild type and injected such mixtures into plants. The combination of $1 / 2 \Delta u m 03848$ X 2/9 WT and 1/2 WT X 2/9 $\Delta u m 03848$ had less virulence when compared to the 1/2 WT X 2/9 WT (Figure 2.10). However, only $1 / 2 \Delta u m 03848$ X 2/9 WT showed significantly reduced virulence compared to WT, and none of the infected plants were able to induce tumors (Figure 2.10). The opposite result was observed when um03848 deleted mutants in FB1 and FB2 strains were used for infection (Figure A.1), showing reduced virulence by FB1 WT X FB2 $\Delta u m 03848$. This shows the complexity of species-specific requirements for nutrients and developmental interaction of fungus to host and the importance of genetic background of strains in determining the degree to which mutations in some genes affect phenotypes.

To understand this reduction of pathogenicity dependent on genetic background, we explored the expression of genes that are involved in the mating pathway as well as high-affinity ammonium transceptor gene, ump2. Although ump2 expression in mutants 
grown on low ammonium was significantly higher than in mutants grown on high ammonium medium, it was downregulated compared to wild type grown on low (Figure $2.12 \mathrm{~A})$. For the mating pathway targets, except pheromone encoding $m f a 2$, all the mating pathway genes were upregulated in the $2 / 9$ mutant (Figure 2.12 B). To be specific, $b E 2$ and prfl genes were significantly upregulated in 2/9 $\triangle u m 03848$ on low ammonium when compared to wild type grown under same conditions. Although cell fusion is governed by the action of pheromones and receptors, the pathogenicity program is controlled by the interaction of homeodomain proteins, $\mathrm{bE}$ and $\mathrm{bW}$ with pheromone response factor (Prf1) as the central regulator ${ }^{126}$. On low ammonium $b E$ serves to prevent an induction of other mating genes such as $m f a$, prfl and $r b f l^{82}$. Therefore, highly increased expression of $\mathrm{bE}$ homeodomain gene and the transcription factor, Prf1 might be the cause of similar levels of virulence for 2/9 $\Delta u m 03848$ compared with WT (Figure 2.10).

The $1 / 2 \Delta u m 03848$ grown on low ammonium showed significantly reduced expression of $b W l$ and null expression of $r b f 1$ (Figure 2.12 B). This result possibly explains the reduced pathogenicity of $1 / 2 \Delta u m 03848$ (Figure 2.10). In addition, the mating targets' expression was also studied in $1 / 2 \Delta u m 03848 \mathrm{c}$, the complementation strain in which a copy of um03848 gene was ectopically expressed in $1 / 2 \Delta u m 03848$ under the control of the Otef promoter. For $1 / 2 \Delta u m 03848 \mathrm{c}$ grown under low ammonium condition, the $b W 1$ and $r b f l$ genes were upregulated, reversing the expression observed in the deleted mutant (Figure 2.12 B). This correlates with the improved virulence of $1 / 2$ Aum03848c compared to the $1 / 2 \Delta u m 03848$ mutant (Figure 2.8). Therefore, we hypothesize that the master control of the pathogenicity in $1 / 2 \Delta u m 03848$ is the level of 
$b W$ gene expression. Combining our genetic study to the established functions ${ }^{82}$, we propose a model for the involvement of the NiR gene, um03848, in $U$. maydis virulence (Figure 2.13). On low ammonium condition, um03848, ump2, $b E$ and $b W$ are independently upregulated; um03848 may be required for this upregulation. $b E$ negatively regulates the expression of transcription factors. However, um03848, ump2 and $b W$, through unknown means, further regulate the expression of transcription factors Prf1 and Rbf1 to control the pathogenic development of the fungus. Additional investigations on the expression of $u m 03848$ in the deletion and overexpression mutants of ump2 and $b$ gene locus will give clearer views on the interaction.

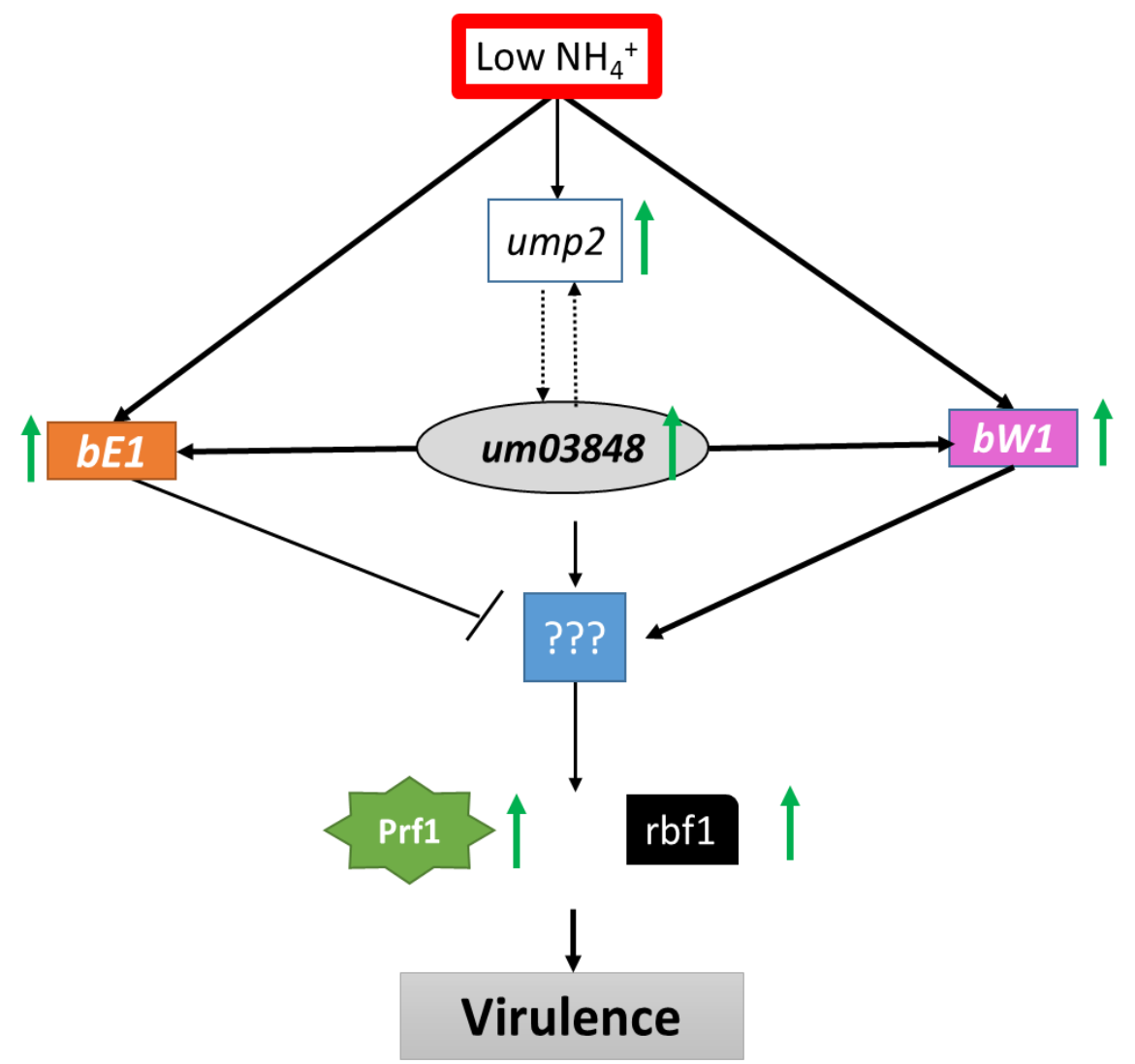

Figure 2. 13 Proposed genetic regulation of um03848 on pathogenicity of U. maydis. 
The interactions between $\mathrm{bE} / \mathrm{bW}$ homeodomain proteins are not involved in mating/cell fusion per se ${ }^{82}$ but are involved in formation of dikaryotic filaments and as an essential regulator for all steps of pathogenesis ${ }^{76,86}$. The $b E$ and $b W$ function independently in haploids to regulate gene expression where $b W$ upregulates the transcription of mating targets such as $m f a l$, prfl and $r b f l^{82}$. In our study, we observed that the downregulation of $b W$ resulted in downregulation of the targets prfl and $r b f 1$ in 1/2 $\Delta u m 03848$. Prf1 is a central regulatory transcription factor that is required for cell fusion and pathogenic development of the fungus and which is regulated posttranscriptionally by different environmental signals ${ }^{126}$. However, Prf1 is not required for conjugation tube formation in the fungus ${ }^{75}$. The $\mathrm{bE} / \mathrm{bW}$ heterodimer directly activates the second transcription factor Rbf1 (regulator of $b$-filament), which is a master regulator for the sexual and pathogenic development in $U$. maydis ${ }^{138}$. Artificial overexpression of either bE-bW or Rbf1 induces the expression of 14 effector genes that are important for the establishment of biotrophic interaction with the host ${ }^{46,139}$. Clpl (clampless 1 ) is a $b$ mating locus dependent gene that is required for the clamp-like structure formation after penetration through the plant cuticle, therefore determining pathogenic development of U. maydis ${ }^{140}$. Clp1 can interact with both bW and Rbf1 separately modulating the pathogenic program. The interaction between Clp1 and bW triggers the $b$ dependent $\mathrm{G} 2$ cell cycle arrest, a prerequisite for the formation of the infectious dikaryon. With Rbf1, Clp1 represses the a-dependent pheromone pathway, conjugation tube formation and the a-induced G2 cell cycle arrest ${ }^{140,141}$. Overall, this emphasizes the complexity of regulation of mating and virulence in $U$. maydis. Therefore, further investigations of the genes involved in different stages of pathogenicity such as appressorium formation, 
establishment of host-pathogen relationship, proliferation inside host and teliospores development will be helpful to understand which phase of the infection cycle is related to um03848.

The biochemical assay was performed on um03848 in this study to analyze its nitrite reductase activity. For this, the $u m 03848$ gene was expressed in yeast and E. coli. Although the expression of um03848 was observed at the transcriptional level, there was no evidence for the presence of um03848 protein in both systems and no enzymatic activity could be demonstrated using a published assay for nitrite reductase activity ${ }^{142}$. The results from this are shown and discussed in Appendix II of the dissertation. 


\section{CHAPTER III}

NITRATE TRANSPORTER, UM03849, GENE IN MATING AND PATHOGENICITY

IN USTILAGO MAYDIS

\section{Introduction}

Fungi can utilize extracellular nitrate after it has been taken up from the medium. This cellular nitrate uptake is an energy-dependent process since it occurs against an electrochemical gradient and thus it requires active nitrate transporter (NRT) ${ }^{143}$. Nitrate transport is the first step for the nitrate assimilation pathway, and it induces the expression of the other genes related to its assimilation. Therefore, the activity of the nitrate transporter is important to understand the regulation of this pathway ${ }^{144}$.

A nitrate transporter [E.C. 3.6.3.26] in fungi was first identified in the filamentous fungus Aspergillus nidulans and was named as $\operatorname{crn} A^{145}$. The availability of sequence alignment tools and the genomic DNA library for different fungi, have opened the study of nitrate transporter in these organisms. The nitrate transport system studied from various fungi have shown that they are able to transport nitrate as well as nitrite and hence is referred to as a nitrate/nitrite transporter ${ }^{100,146,147}$. Nitrate transporters in Escherichia coli and the denitrifying bacterium, Pseudomonas aeruginosa, are involved in excretion of nitrite produced by the dissimilatory reduction of nitrate. In case of low concentrations of extracellular nitrate, the nitrate transporters in these bacteria mediate 
nitrite excretion as a nitrate/nitrite antiporter. This process prevents the intracellular accumulation of toxic levels of nitrite before action of nitrite reductase ${ }^{148-150}$. However, this specific function of nitrate transporter hasn't yet been reported in fungi.

Expressed sequence tag and transcript analyses in U. maydis performed by Ho, Cahill, and Saville 2007 have shown that the nitrate transporter gene, um03849 (or $U M A G \_11105$ ), is highly up regulated in the nitrogen starved condition. Even though a nitrate transporter gene has been identified in $U$. maydis, its effect on the phenotype of the fungus has not yet been explored. The study discussed in this Chapter is to understand the role of NRT in $U$. maydis. For this, the nitrate transporter gene, um03849, was edited by two different techniques. The first technique was by utilizing the homologous recombination mechanism of $U$. maydis whereby um03849 was replaced with a hygromycin resistance (HygR) gene cassette. This results in the deletion of the functional copy of the um03849 gene in the fungus. The second method was by using the CRISPRCas9 genome editing technique followed by the non-homologous end joining (NHEJ) repairing mechanism of fungus. This results in INDEL (insertion-deletion) mutations in the um03849 gene in the fungus. By either of the gene editing techniques, um03849 gene mutation was obtained in the compatible mating partners, $1 / 2$ and 2/9. The morphological observations were analyzed for um03849 mutants and compared to their respective wild type strains. The morphological observations included the ability of mutants to grow on different nitrogen source media and in different stressor media; the ability to mate with its opposite compatible partner and cause virulence to the host plant were also examined.

The um03849 deleted $U$. maydis strains had reduced growth in nitrate medium and reduced virulence to the plant. The CRISPR-Cas9 mediated um03849 mutants in $U$. 
maydis showed a background specific effect on growth on nitrate medium. The frameshift and substitution mutations in um03849 resulted in reduced growth in different nitrogen sources and reduced virulence to plants. However, an in-frame mutation in um03849 didn't show any difference in growth and virulence compared to respective wild types. The mating target gene expression analysis in $\Delta u m 03849$ showed differential regulation of the targets.

\section{Methods}

Strains and growth conditions. The $U$. maydis strains used in this study are listed in Table 3.1. Escherichia coli strain DH5a (Thermo Fisher) was used for all cloning and plasmid maintenance. U. maydis strains were grown at $27^{\circ} \mathrm{C}$ in solid or liquid YEPS, PDA, HSS media (Brachmann, König, Julius, \& Feldbrügge, 2004) or Holliday Salt media (Holliday, 1974). Strains grown in liquid media were grown overnight shaking at $250 \mathrm{rpm}$. E. coli cells were grown in LB medium supplemented with $200 \mu \mathrm{g} / \mathrm{mL}$ ampicillin at $37^{\circ} \mathrm{C}$.

Table 3. 1 Strains used in Chapter III.

\begin{tabular}{|c|c|c|}
\hline Ustilago Strain & Genotype & Reference \\
\hline $1 / 2$ & al bl & Gold et al., 1997 \\
\hline 2/9 & $a 2 b 2$ & Gold et al., 1997 \\
\hline 1/2 $\Delta u m 03849-H y g R$ & alb1 um03849:: hyg ${ }^{R}$ & This study \\
\hline 2/9 Dum03849- HygR & $a 2 b 2$ um03849:::hyg ${ }^{R}$ & This study \\
\hline Plasmid & Genotype & Reference \\
\hline pum03849-HygR & up $-h y g^{R}-$ down $a m p^{R}$ Ori & This study \\
\hline \#1779 pCas9HF1 & $a m p^{R}$ pU6-phsp70-Cas $9 c b x^{R}$ & Zuo, Depotter and \\
\hline \#1782 pCas9-bW1 & $a m p^{R} \mathrm{pU} 6-b W 1$-scaffold phsp70-Cas 9 & Doehlemann, 2020 \\
\hline pCas9-um03849 & $\begin{array}{l}a m p^{R} \mathrm{pU} 6-\text {-um03849 phsp70-Cas9 } \\
c b x^{R}\end{array}$ & This study \\
\hline
\end{tabular}


Primers design and PCR. Primers (Table 3.2) were designed and PCR reactions were performed as described previously (Chapter II).

Table 3. 2 List of primers used in Chapter III.

\begin{tabular}{ll}
\hline Primers & Sequence \\
\hline New AmpFw 3849T & tgaccagcaatcTTAATTAATATTGAAAAAGGAAGAG \\
OriAmp R-3849UpT & ggttgtttgcGATATCAAAAGGCCGCGTTG \\
3849UpFwd- Ori T & TTTTGATATCGCAAACAACCAAGCAAGCTG \\
3849UpRev HygT cir & cgtcgtttaAGGAGATAAGACCGTAGCGC \\
HygRFw 3849UpT cir & CTTATCTCCTTAAAACGACGGCCAGTGAAT \\
HygRcir Rev-3849DnT & TTCTCTACAGTGTGGAATTGTGAGCGATA \\
3849DnFw-HygT cir & acaattccacaCTGTAGAGAAGTGGGGTGT \\
New-AmpT-3849DnR & ccttttcaatattaattaaGATTGCTGGTCACATTGGCT \\
um_New3849Fw - & CAAAATTCCATTCTACAACGggaaagccaatcagaagaa- \\
pU6T & -GAAGTTTAGAGCTAGAAATAGCA \\
phsp70 Fw Cas9T & cttcttaggcggcatggatcCTGTGAAATCTAAAACTTTTGC \\
\hline
\end{tabular}

Vector construction and Genetic manipulation. The deletion of um03849 in U. maydis was obtained by homologous recombination as described previously ${ }^{113}$. To create the deletion construct of um03849, first, the $1 \mathrm{~kb}$ flanking regions upstream and downstream of the um03849 gene from FB1 strain, hygromycin resistance cassette from plasmid pUMa1507 ${ }^{114}$ and ampicillin resistance along with Ori cassette from plasmid pMS73 ${ }^{151}$ were amplified using primers in table 4.2 and secondly performed using the Gibson overlap-technique ${ }^{117}$ to obtain a vector designated as pum03849-HygR, which was then transformed into commercially competent $E$. coli. Sequencing from the middle of $\mathrm{HygR}$ towards 3' and 5' regions showed the correct orientation of flanks and HygR gene in the plasmid. The pum03849-HygR plasmid confirmed via sequencing was used as a template in PCR using flanks primers to obtain the linearized $u m 03849:: H y g^{R}$ construct. The construct was then transformed into U. maydis strains by the polyethylene glycol/ protoplast method ${ }^{113,116}$ which would replace um03849 ORF with the hygromycin 
resistance cassette. Transformed $U$. maydis were selected on YEPS agar plates containing $150 \mu \mathrm{g} / \mathrm{ml}$ hygromycin B (Gold Biotech). Successful transformation was confirmed by PCR and gene disruption was further confirmed by Real time quantitative PCR (qRTPCR) (Figure A.10).

sgRNA design and construction of Cas9 vector with um03849 target. The targeting single guide RNA (sgRNA) for the gene um03849, was designed using E-CRISP design tool (http://www.e-crisp.org/E-CRISP/index.html) ${ }^{152}$. On the E-CRISP webpage, Ustilago maydis was selected as the organism for genome comparison and the targeted, um03849, genomic DNA sequence in FASTA format was provided. Once the search for sgRNA was completed, the target sequence with the best scores and most towards Nterminal was selected. The sgRNA was ordered as primer, um_New3849Fw - pU6T, which contains 20 bp um03849 targeted sgRNA along with 20 bp scaffold RNA at the 3' end and $20 \mathrm{bp}$ U6 promoter at the 5' end. This primer along with phsp70Rev was used to amplify RNA scaffold-sgRNA and phsp70 promoter from plasmid obtained from \#1782 (Table 3.1). The amplified product was assembled with linearized \#1779 plasmid at the Acc65I restriction site using Gibson assembly technique ${ }^{117}$. The pCas9-um03849 plasmid, generated by the Gibson assembly, was transformed into competent $E$. coli cells. The successful cloning was identified by sequencing. The isolated plasmid was then used to transform compatible $U$. maydis mating partners, $1 / 2$ and $2 / 9$ strains. Transformed $U$. maydis were selected on YEPS agar plates containing $3 \mu \mathrm{g}$ carboxin per ml (Santa Cruz Biotechnology, Dallas, TX). Selected colonies were sub-cultured in YEPS agar without carboxin to eliminate the plasmids bearing Cas9. Such putative transformed strains now lacking Cas9 were then sequenced at the targeted region of the gene. 
Cell growth, nitrogen utilization, stress tests, mating and pathogenicity. Cell growth rates, utilization of different nitrogen sources, cell wall stress tests, mating assays and plant infections were performed as described previously (Chapter II).

\section{Results}

Induction of nitrate transporter gene, um03849, on low ammonium. The qRT-PCR was performed on the wild type strains that were grown on low and high ammonium medium for $48 \mathrm{~h}$. The expression of the um03849 gene in wild type grown in low ammonium was compared to their respective strain grown in high ammonium. The expression of um03849 gene was significantly increased when wild type strains were grown in the ammonium starved condition (Figure 3.1) compared to those grown in high ammonium condition. This observation led us to investigate the role of NRT, um03849, in the phenotype of $U$. maydis in terms of mating and virulence to its host plant.

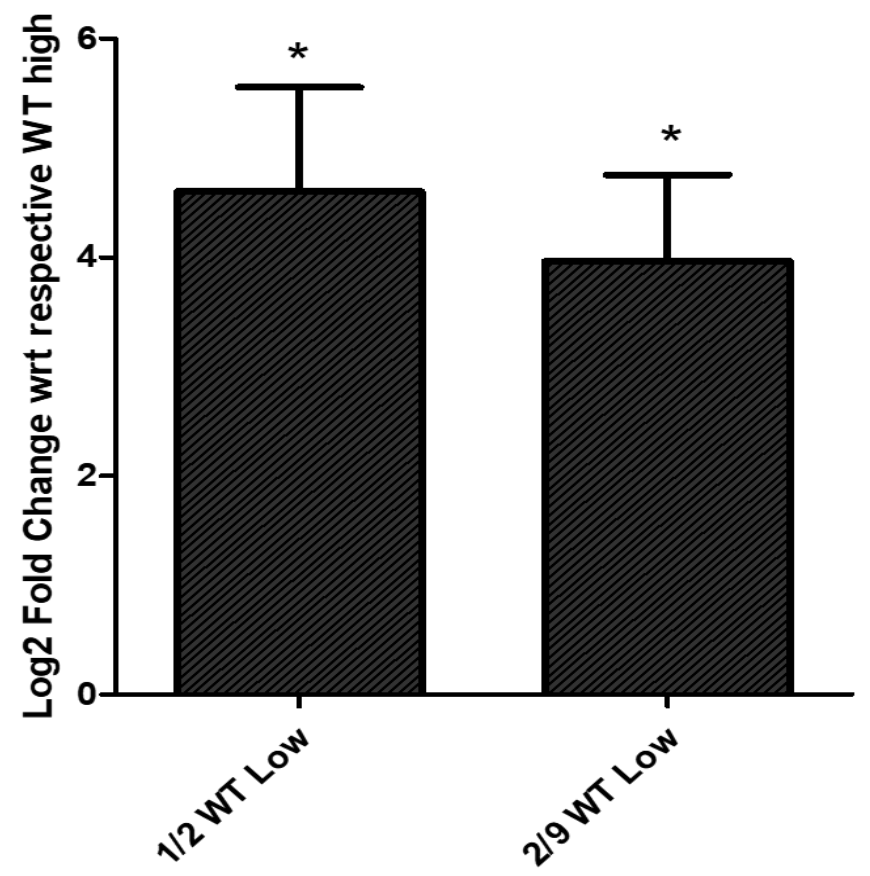

Figure 3. 1 Relative expression of um03848 in wild type grown under low ammonium conditions.

Bars represent the averages of biological triplicates and standard errors are indicated on top of each bar. Statistical analysis was done using Student's t-test (unpaired two-tailed) in GraphPad Prism 7.0 with respect to (wrt) respective wild type grown on high 
ammonium i.e., 1/2 WT low was compared to $1 / 2 \mathrm{WT}$ on high and 2/9 on low was compared to 2/9 WT grown on high. * indicates the significance with p-value $<0.05$.

\section{Deletion of um03849 (NRT) does not affect filamentation in low ammonium media.}

The haploid filamentation formation was observed for wild type strains $1 / 2$ and 2/9 and the $\Delta u m 03849$ mutant in these compatible mating backgrounds. All strains were streaked on low ammonium medium and the ability to form haploid filaments was observed after the incubation of plates for 4 days at $28{ }^{\circ} \mathrm{C}$. The $\Delta u m 03849$ showed smaller colony size but was equally filamentous as that of their respective wild type strains (Figure 3.2).

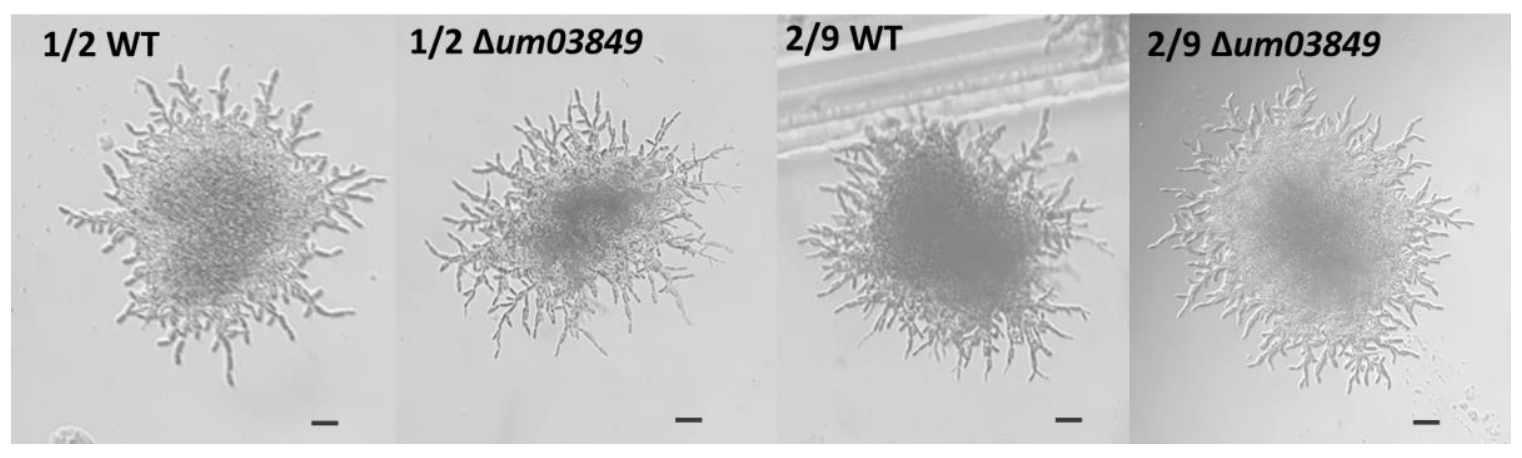

Figure 3. 2 Phenotype observation of $\Delta$ um03849 grown on low ammonium medium. The filamentation assay was performed with haploid cells (labeled) grown on low ammonium medium for 4 days. Size bars, $100 \mu \mathrm{m}$.

The deletion of um03849 does not affect the growth of fungus in the medium with different nitrogen sources. The deletion mutants were grown in different nitrogen source media. The minimal nutrient medium was incorporated with ammonium, urea and nitrate or without any nitrogen. Deletion mutants grew in a similar way to their respective wild type strains in all the media, except for 2/9 $\Delta u m 03849$ mutant (Figure 3.3). 
(A) $\underline{\text { AMM }}$

(B) Urea

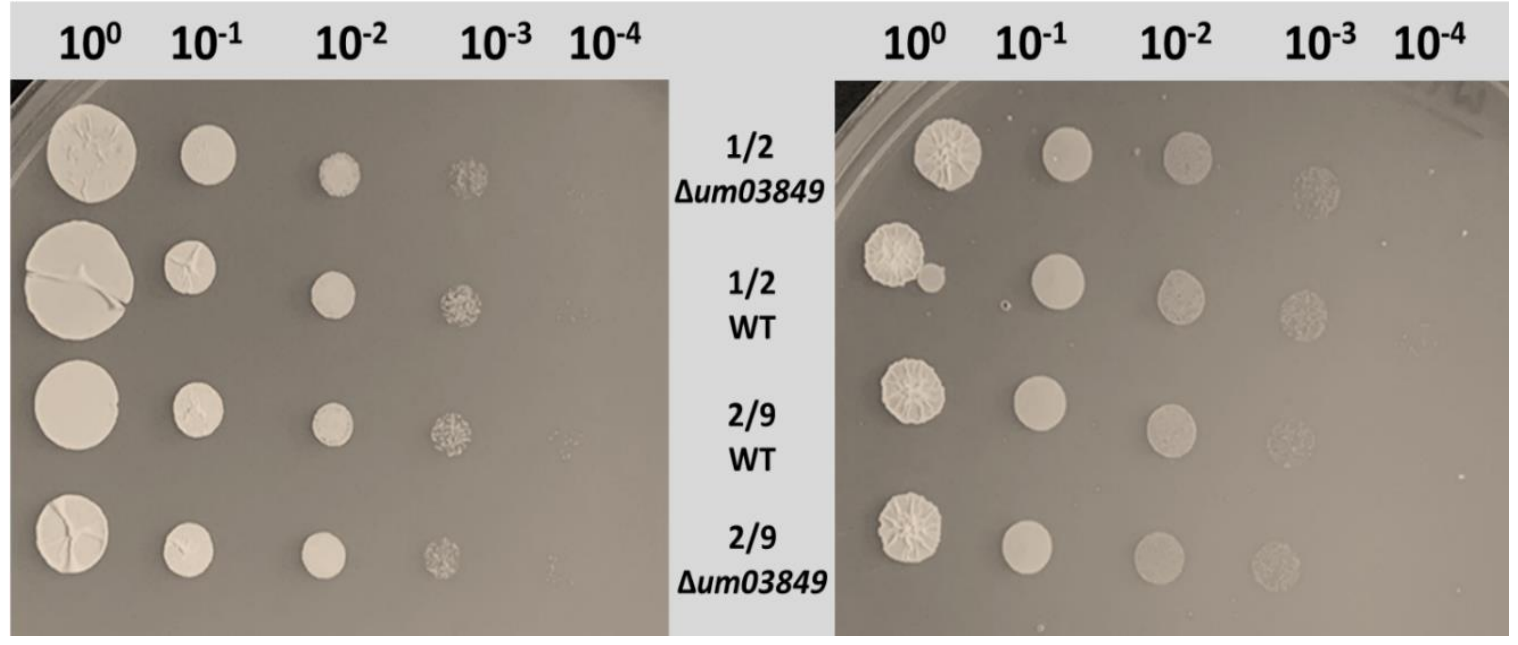

(C) $\underline{\mathrm{NO}}_{3}^{-}$

(D) $\underline{-\underline{N}}$

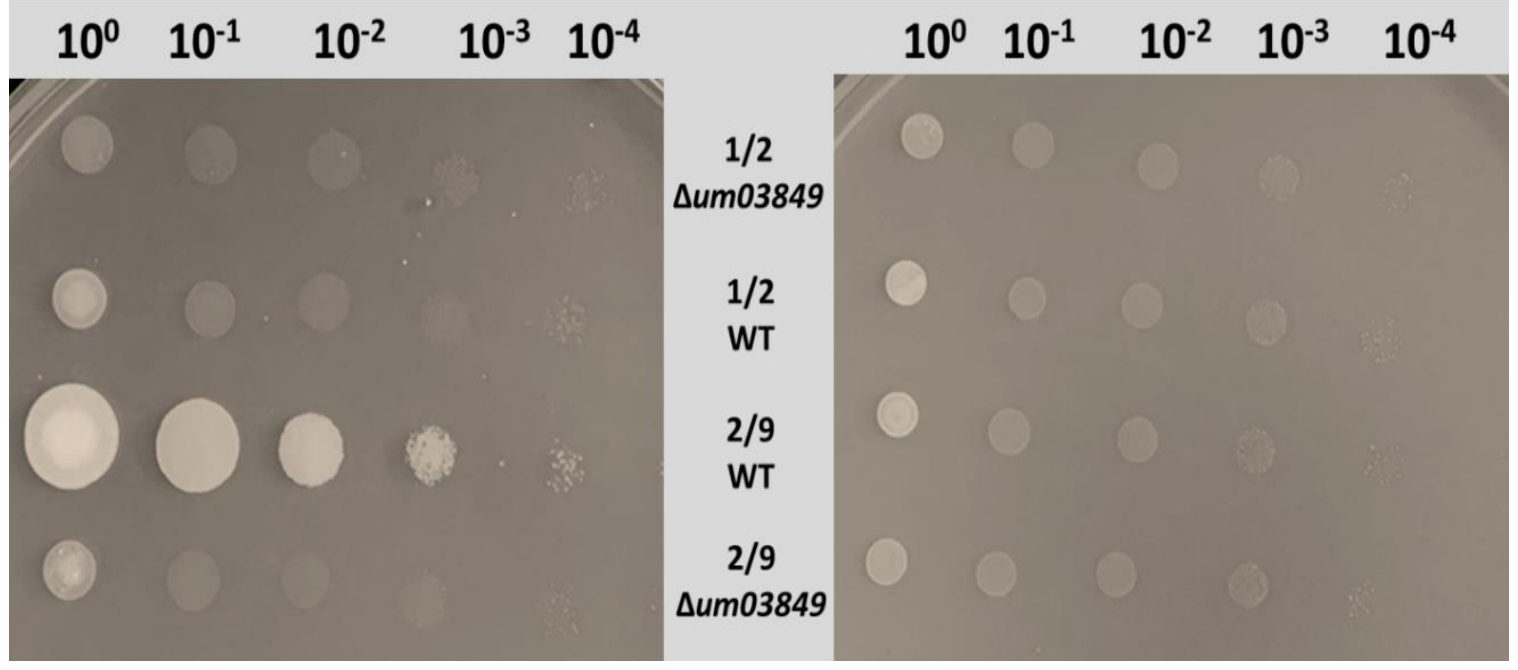

Figure 3. 3 Nitrogen utilization by $\Delta$ um03849.

The 10-fold serial dilutions of wild type and $\Delta u m 03849$ strains were spotted onto minimal media incorporated with (A) $0.3 \% \mathrm{w} / \mathrm{v}(\sim 23 \mathrm{mM})$ Ammonium Sulphate (AMM), (B) 0.3 $\%$ w/v ( 50 mM) Urea, (C) $0.3 \% \mathrm{w} / \mathrm{v}(\sim 30 \mathrm{mM})$ Potassium Nitrate $\left(\mathrm{NO}_{3}{ }^{-}\right)$and (D) no nitrogen $(-\mathrm{N})$. Plates were grown at $28{ }^{\circ} \mathrm{C}$ for $48 \mathrm{hrs}$. Mutants were compared with their respective wild type. 2/9 WT unexpectedly grow better on nitrate than other strains.

However, there was not any difference in the growth of mutants to that of their respective wild type strains on any other medium. 
The deletion of um03849 has a slight effect on the growth of $U$. maydis in different stress media. The $\triangle u m 03849$ strains were grown in a YEPS supplemented with $1 \mathrm{M}$ sorbitol or $1 \mathrm{M} \mathrm{NaCl}$ as high osmotic media as well as YEPS supplemented with $1 \mathrm{M}$ Congo red (CR) as cellular stress medium affecting cell wall and YEPS supplemented with $10 \mathrm{mM}$ hydrogen peroxide $\left(\mathrm{H}_{2} \mathrm{O}_{2}\right)$ as oxidative stress. The growth of mutants was compared to their respective wild type strains grown in the same conditions for $48 \mathrm{~h}$. The sum03849 showed slightly reduced growth in medium with sorbitol and Congo red, but this difference was only visible at the highest dilution (Figure 3.4).

\section{(A) $\underline{\mathrm{NaCl}}$}

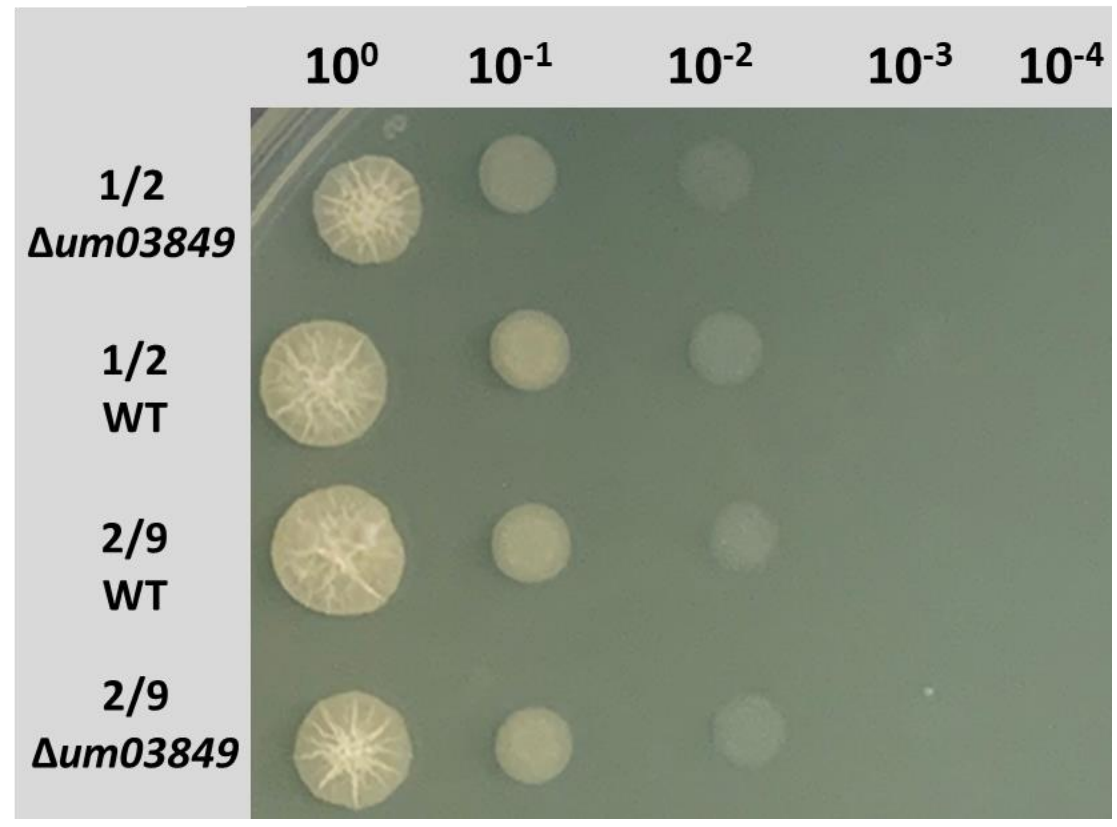


(B) $\underline{\mathbf{H}}_{2} \underline{\mathbf{O}}_{2}$

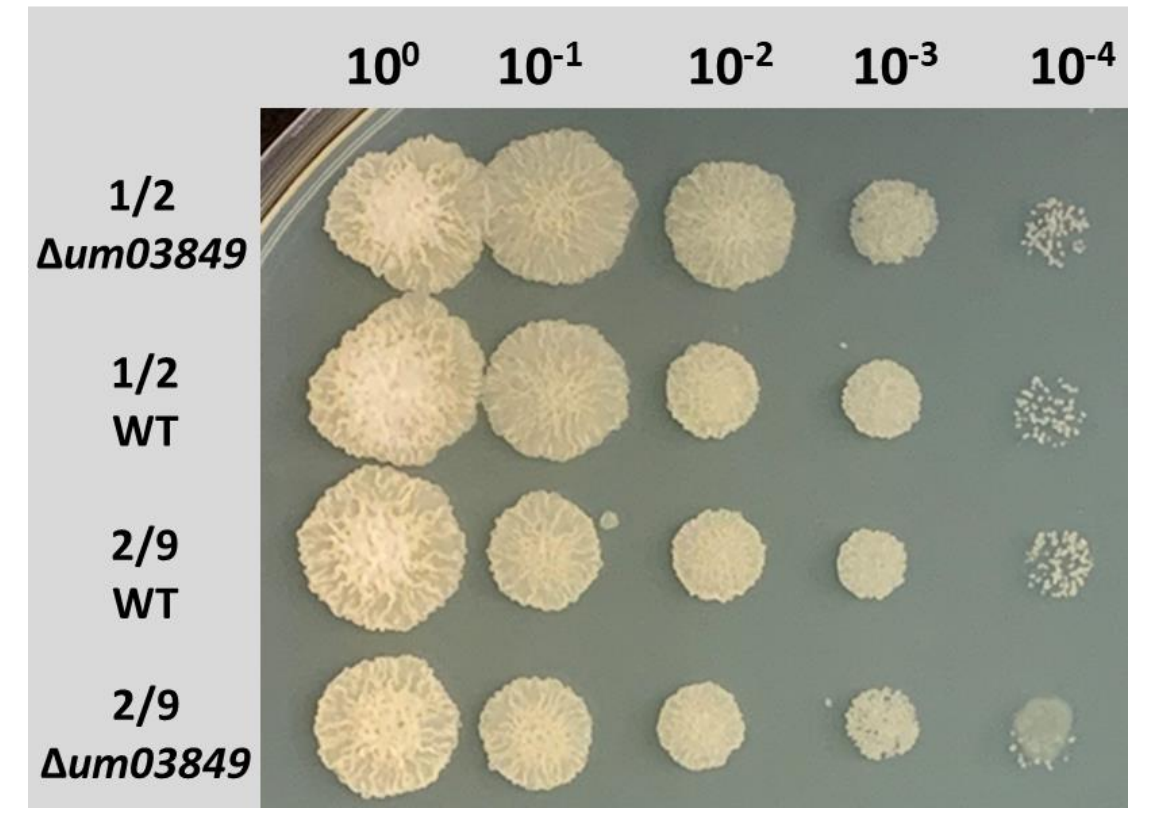

(C) $\underline{\mathrm{CR}}$

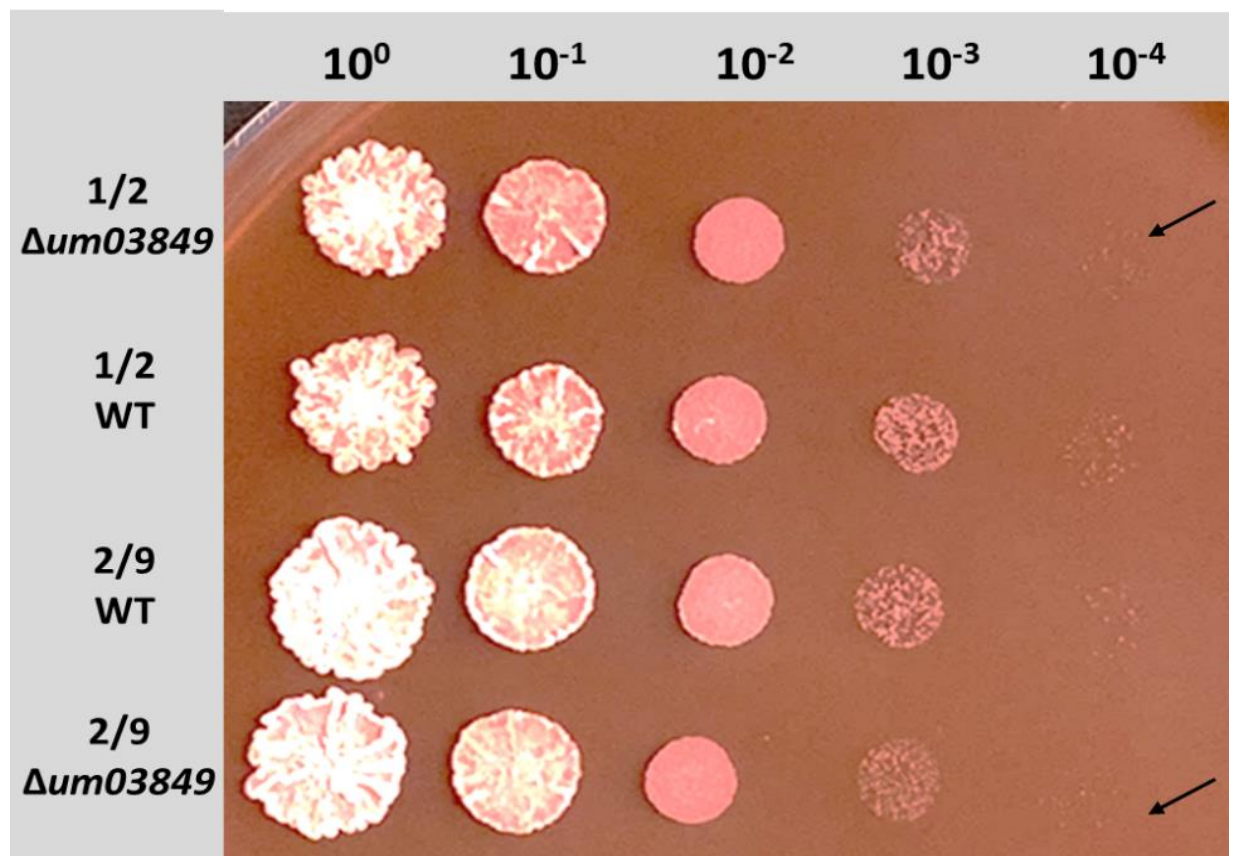


(D) Sorbitol

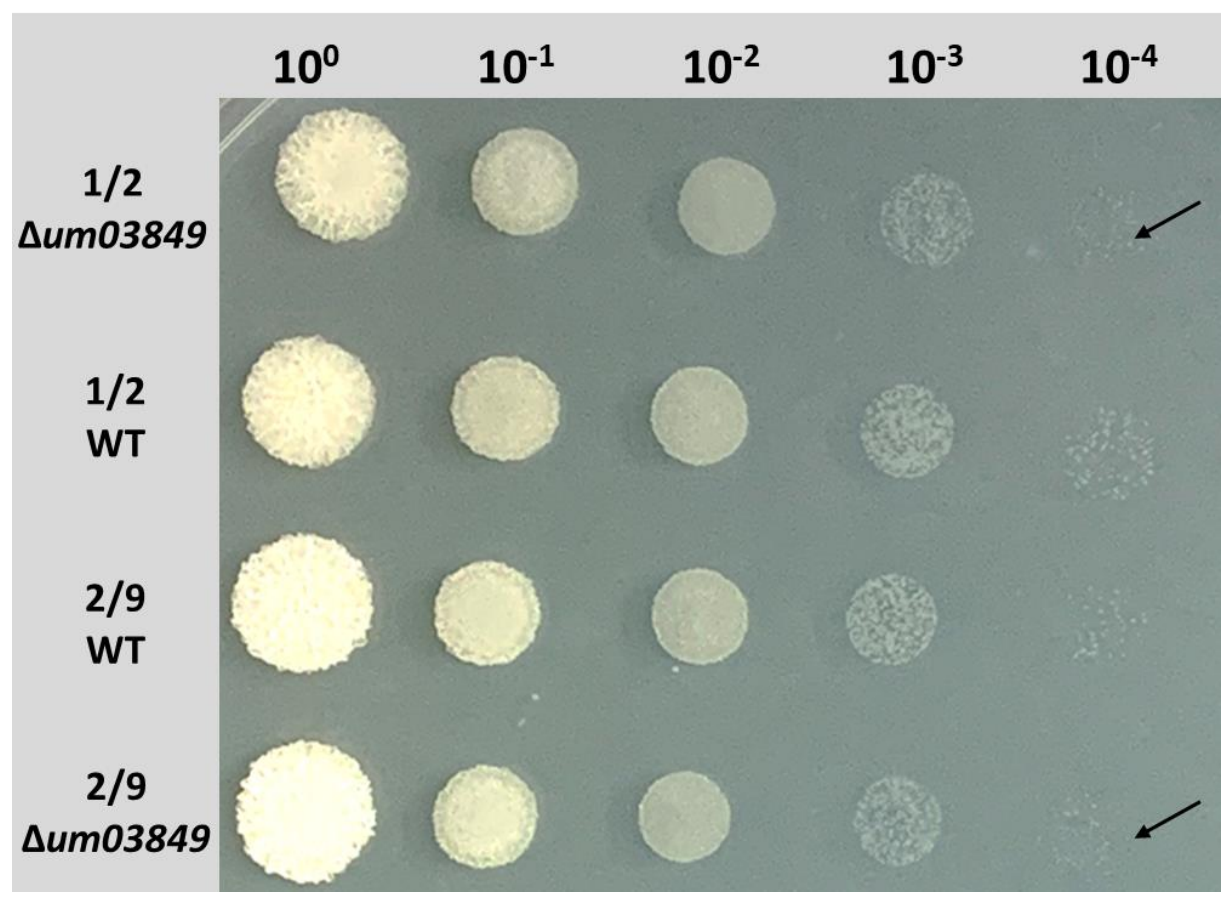

Figure 3. 4 The growth of $\Delta$ um03849 in different stress media.

The 10-fold serial dilutions of wild type and $\Delta \mathrm{um} 03849$ strains were spotted onto YEPS supplemented with (A) $1 \mathrm{M} \mathrm{NaCl}$ or (B) $10 \mathrm{mM} \mathrm{H}_{2} \mathrm{O}_{2}$ or (C) $1 \mathrm{mM}$ Congo Red (CR) or (D) $1 \mathrm{M}$ Sorbitol. The $\mathrm{NaCl}$ and Sorbitol are osmotic stress, CR is cellular stress (acting on cell wall $)$ and hydrogen peroxide $\left(\mathrm{H}_{2} \mathrm{O}_{2}\right)$ is an oxidative stress. Plates were grown at $28{ }^{\circ} \mathrm{C}$ for $48 \mathrm{hrs}$. Mutants were compared with their respective wild type. Black arrows indicate a slight difference in the growth of mutants to that of their respective wild type strains on sorbitol and CR plates.

\section{The deletion of um03849 has no obvious effect on the fungus' ability to form}

dikaryotic filaments with its opposite partner. To assess the ability of $\Delta u m 03849$ to

form dikaryotic aerial hyphae, the haploid strains of opposite mating type were grown

overnight to the exponential phase. The strains were pelleted, washed with water and premixed with the opposite partner in a tube. The pre-mixed strains were then spotted onto PDA medium incorporated with $1 \%$ charcoal. The $\Delta u m 03849$ showed the characteristic white "fuzz" phenotype with its opposite mating partner as well as with the opposite wild 
type strains (Figure 3.5). This indicates that the deletion of um03849 doesn't affect the ability of fungus to mate with its compatible partner.

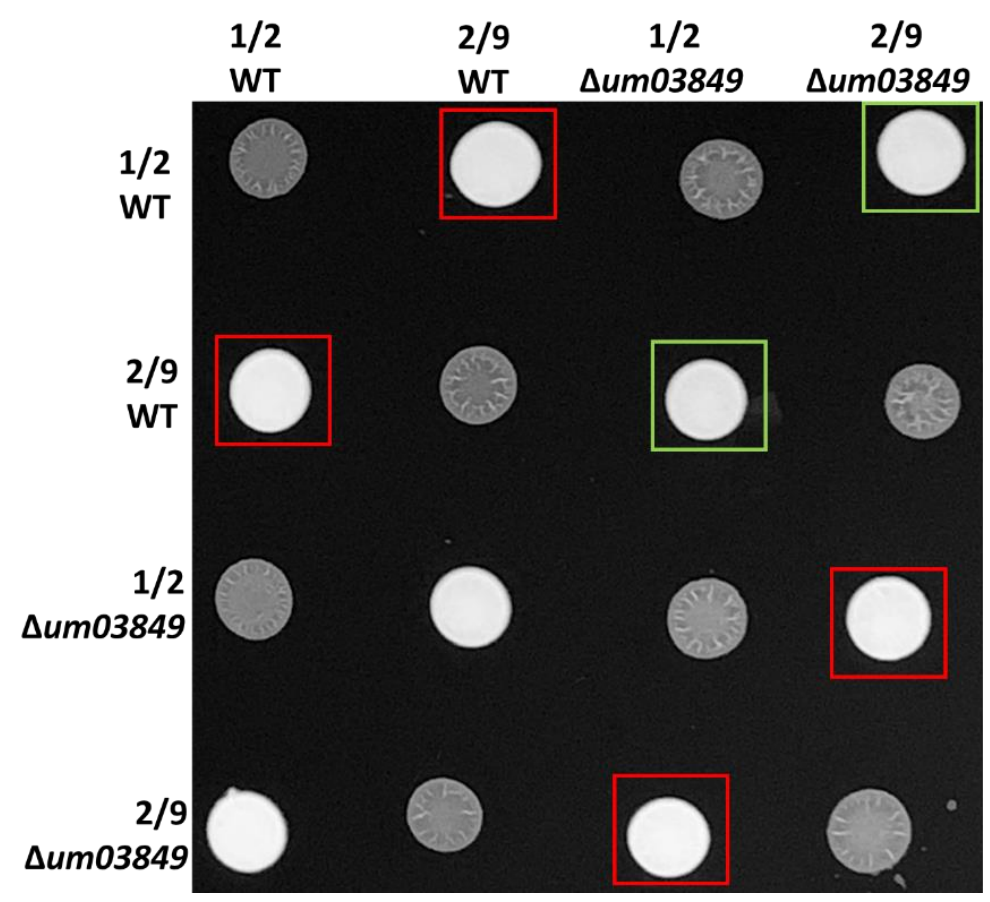

Figure 3. 5 Mating assay of $\Delta$ um03849 on PDA-charcoal plate.

Equal mixtures of $1 / 2$ or $2 / 9$ wild type or corresponding $\Delta u m 03849$ haploid strains were pre-mixed in a tube and spotted onto PDA plate and grown for $24 \mathrm{hrs}$. A positive mating reaction produced a white "fuzz" phenotype of aerial hyphae production. Red boxes indicate the successful mating of wild type (1/2 X 2/9) or $\Delta u m 03849$ (1/2 X 2/9) strain. Green boxes show the characteristic white "fuzz" phenotype by $\Delta u m 03849$ with their compatible mating wild type partners.

The deletion of um03849 affects the pathogenesis to the host plant. The compatible opposite mating strains of $\Delta u m 03849$ were pre-mixed and injected into one-week-old maize seedlings to determine their ability to infect host plants. The $\Delta u m 03849$ was also pre-mixed with opposite wild type mating partner to evaluate if functional copies of um03849 are required in both partners for successful infection and complete virulence. The degree of pathogenicity in the plants was then compared to that infected with both wild type mating partners. The $\Delta u m 03849$ (1/2 X 2/9) showed significant reduction in 
virulence to the plant (Figure 3.6). The combination of 1/2 WT X 2/9 $\Delta u m 03849$ also showed significant reduction in virulence to the plant with less than $15 \%$ of the plants showing the formation of galls. However, the plants infected by $1 / 2 \Delta u m 03849$ X $2 / 9$ WT showed higher virulent symptoms than that infected by $1 / 2 \Delta u m 03849$ X 2/9 $\Delta u m 03849$ but lesser death of plants than plants infected by both wild type partners (Figure 3.6).

Furthermore, the effect of $\Delta u m 03849$ on the pathogenicity to the plant was measured by comparing the height of plant and number of leaves relative to uninfected plants or those infected by both wild type partners. Difference in the measurements between 8 dpi (days post infection) and 0 dpi is depicted in the figure 3.7. These differences in the plants infected with $\Delta u m 03849$ strains were compared with those infected with the wild type strains. The plants infected with $\Delta u m 03849$ grew significantly taller (Figure 3.7 A) and had more leaves (Figure 3.7 B), indicating reduced infection, compared to plants infected by wild type strains. Although, 1/2 WT X 2/9 $\Delta u m 03849$ showed taller plants and more leaves, the trend towards reduced virulence was not statistically significant ( $\mathrm{p}$-value $=0.06$ ). This was in contrast to the significant reduction in virulence on the plant by $1 / 2$ WT X 2/9 $\Delta u m 03849$ as seen in figure 3.6. 


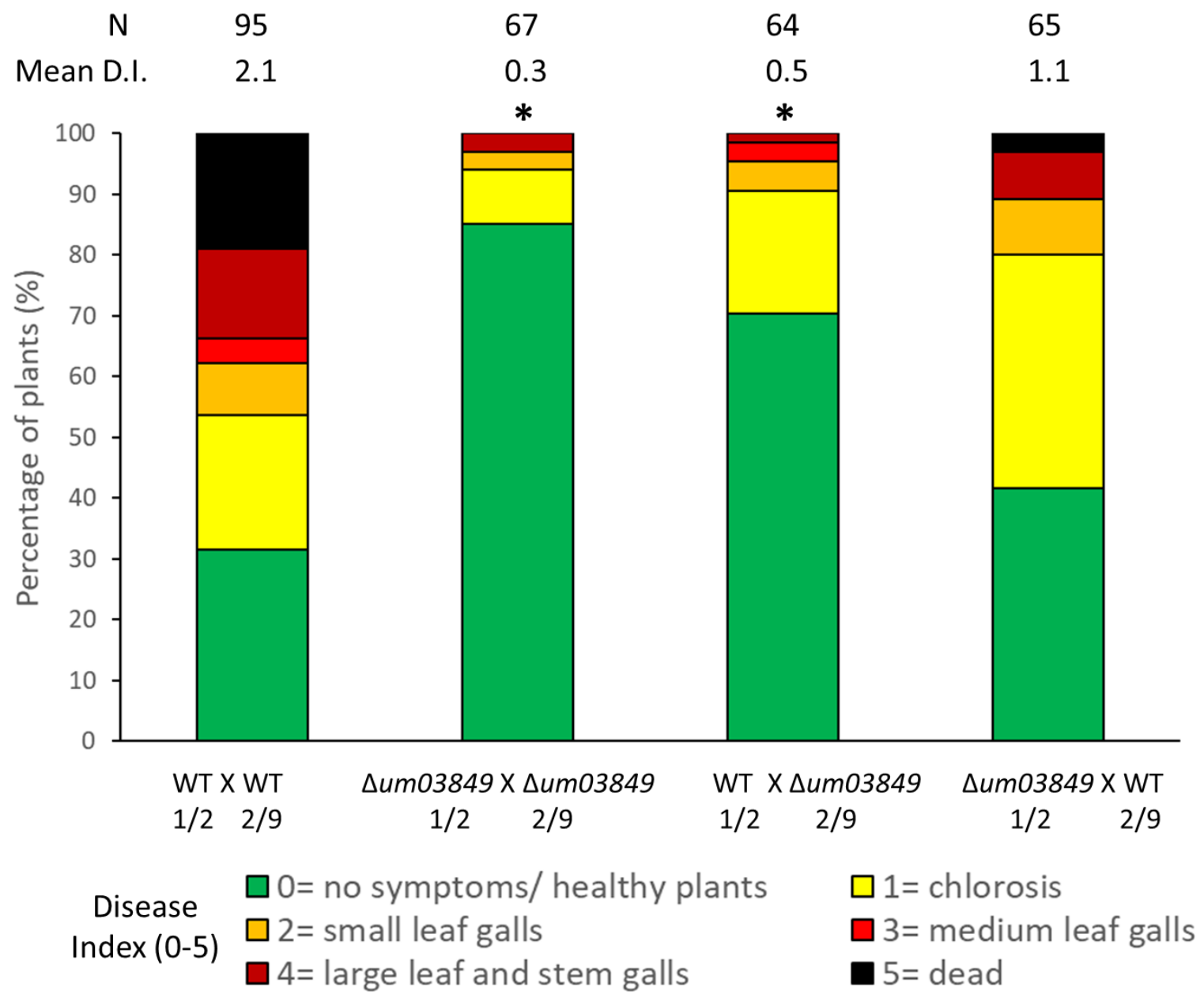

Figure 3. 6 Plant pathogenicity assay for $\Delta u m 03849$.

The indicated strains of $U$. maydis were pre-mixed and injected into 7-day-old maize seedlings. Disease symptoms were scored 14 days post infection (dpi). The graph displays the percentage of plants with specific symptoms of infection. The $\mathrm{X}$ axis indicates the paired $(1 / 2 \times 2 / 9)$ background that is injected into maize plant. The $\Delta u m 03849$ strains were combined with the wild type strain of opposite mating-type and were compared with infections due to both wild type (1/2 WT X 2/9 WT). The disease symptom was categorized from 0 to 5 as depicted at the bottom of the figure. Numbers on the top represents the number of plants infected and mean for disease indices (DI). The data were analyzed using Kruskal-Wallis test followed by post-hoc comparison and an asterisk $(*)$ indicates significant difference $(\mathrm{p}<0.05)$ compared to those where both partners were wild type strains $(1 / 2 X 2 / 9)$. The significant reduction $(\mathrm{p}<0.05)$ of plant pathogenesis was seen in $\Delta u m 03849(1 / 2 X 2 / 9)$ and 1/2 WT X 2/9 $\Delta u m 03849$. 


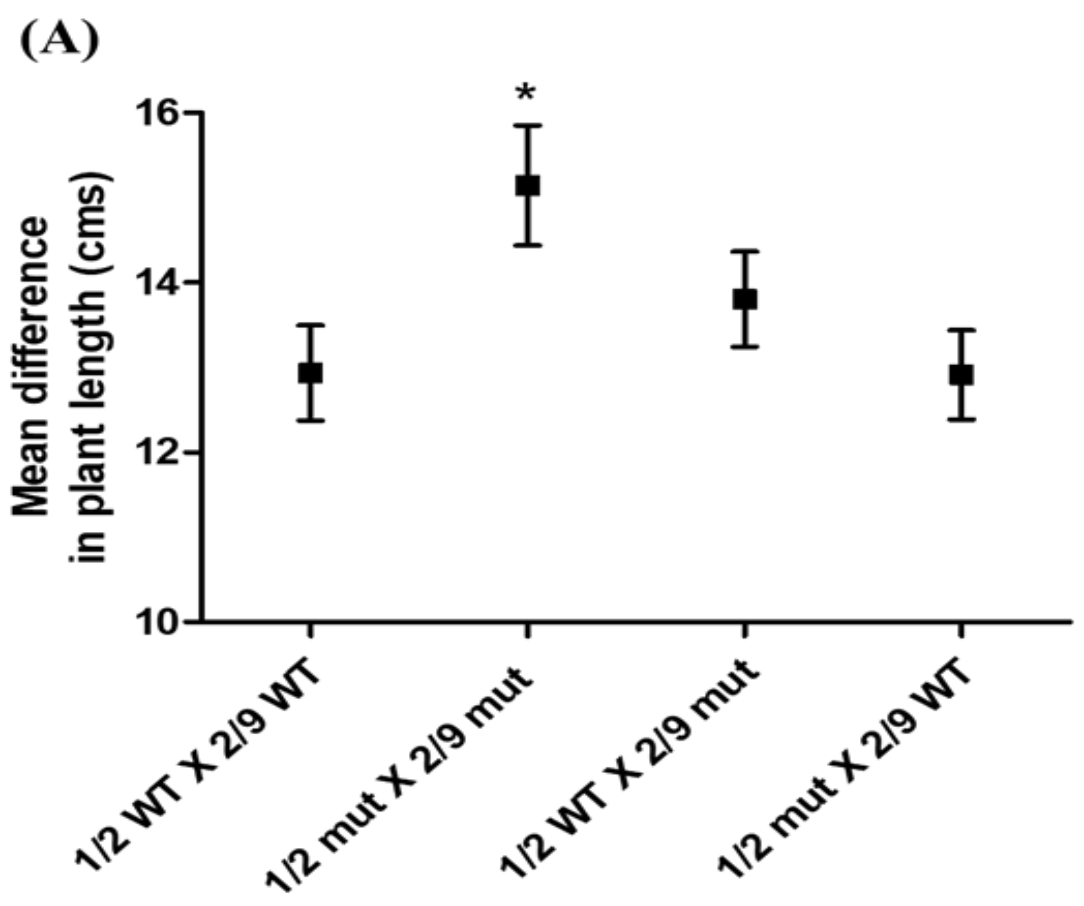

(B)

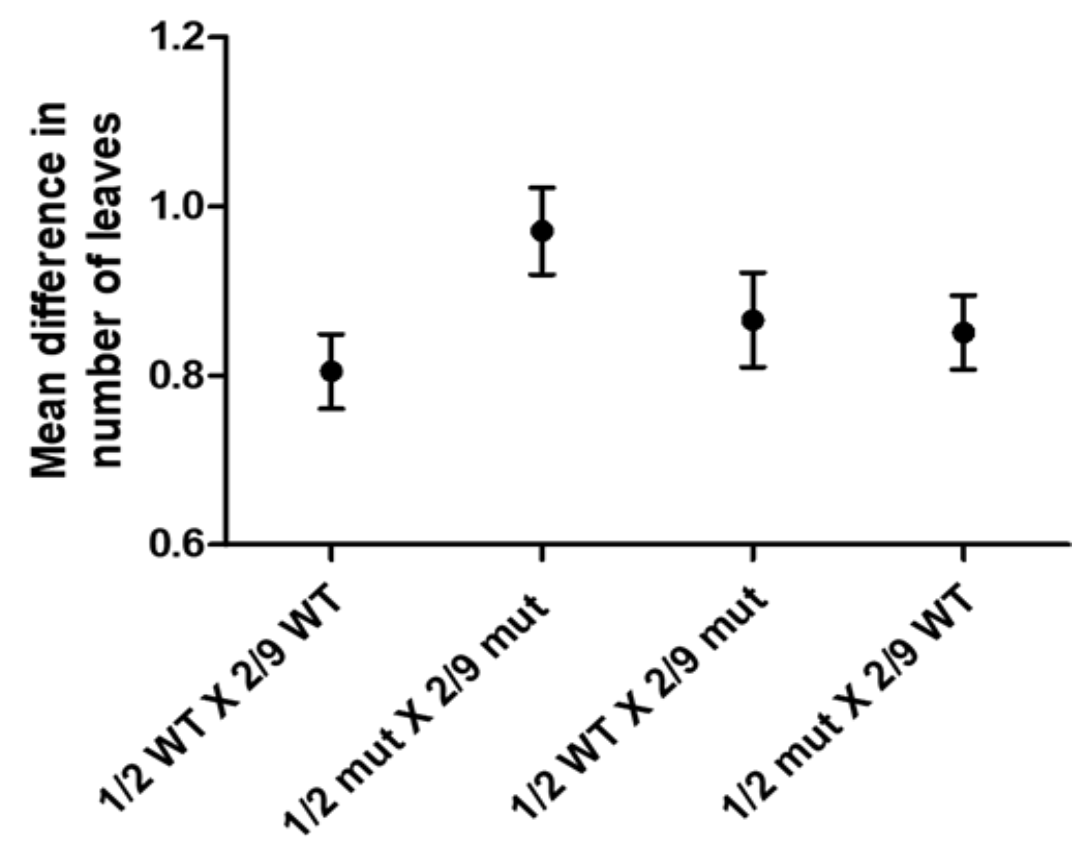

Figure 3. 7 Plant growth measurements of the plants infected by $\Delta$ um03849.

Effect of $\triangle u m 03849$ on (A) plant height (cms) and (B) number of leaves are shown in the graph. The plants were infected with wild type (1/2 X 2/9), $\Delta u m 03849$ (1/2 X 2/9) and $\Delta u m 03849$ crossed with compatible wild type partner, as shown on $\mathrm{X}$-axis. The Y-axis represents the mean difference in the length and number of leaves for the plants grown at 8 dpi vs 0 dpi. Statistical analysis was done by ANOVA followed by Tukey's test in 
GraphPad Prism comparing each group to the wild type ( $1 / 2 X 2 / 9)$. P value $<0.05$ was considered significant. The asterisk $(*)$ in the figure shows that the plants infected with $\Delta u m 03849$ (1/2 X 2/9) have significant increase in plant height and number of leaves when compared to that infected by wild type. However, plants infected by $\Delta u m 03849$ with its opposite wild type strain didn't have significant difference in plant height or leaf count.

\section{The deletion of um03849 yields differential gene expression of mating targets under}

low ammonium conditions. To evaluate the effect of $\Delta u m 03849$ on the virulence of the strains to plants, the expression of mating targets and ump 2 expression was assessed by qRT-PCR in $1 / 2$ and 2/9, and for $\Delta u m 03849$ mutants of the respective strains. Both $1 / 2$ $\Delta u m 03849$ and 2/9 $\Delta u m 03849$ showed significant downregulation of genes in the mating pathway and ump2 (Figure 3.8). This might be the reason for reduced virulence of Dum03849 mutants.

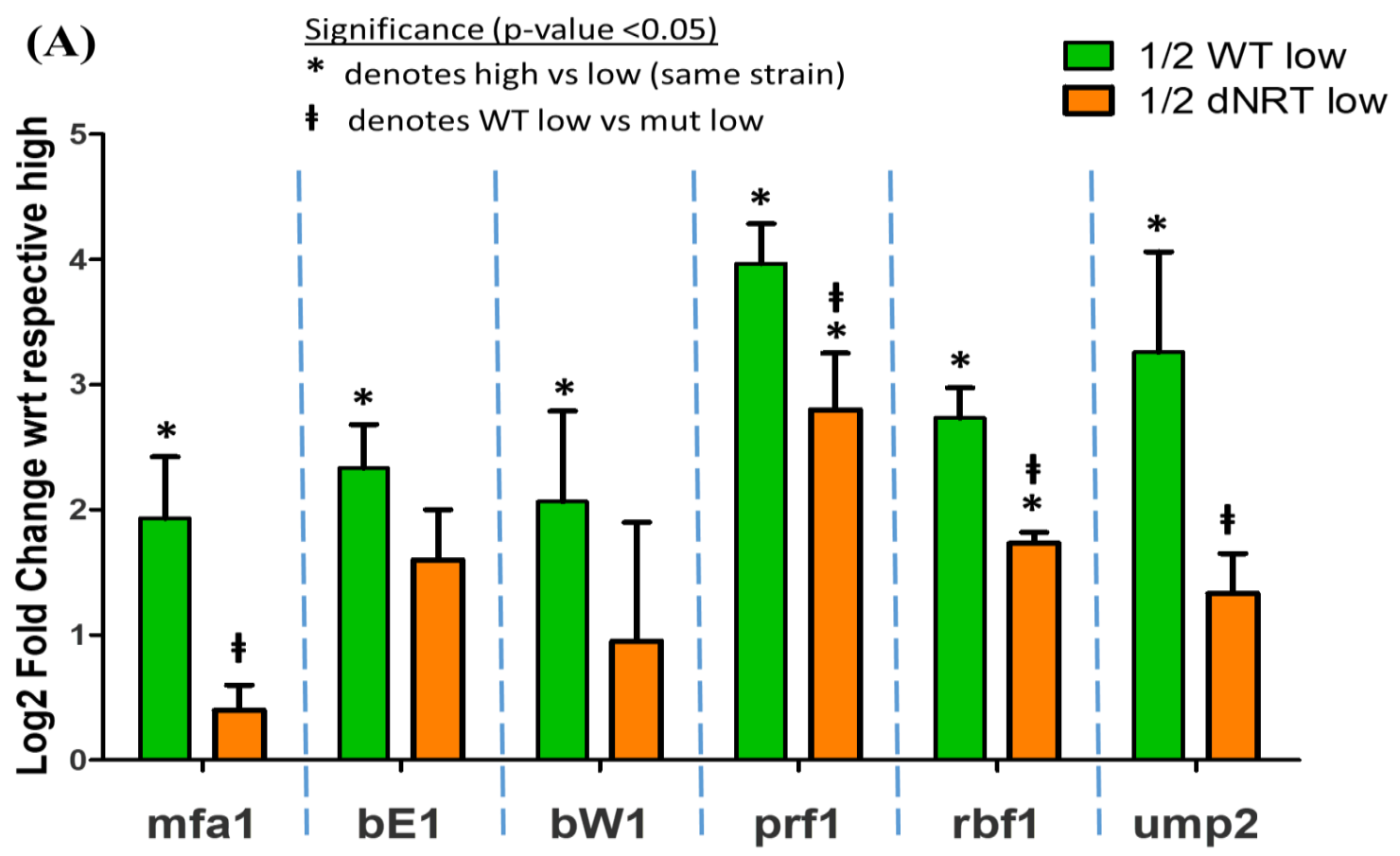



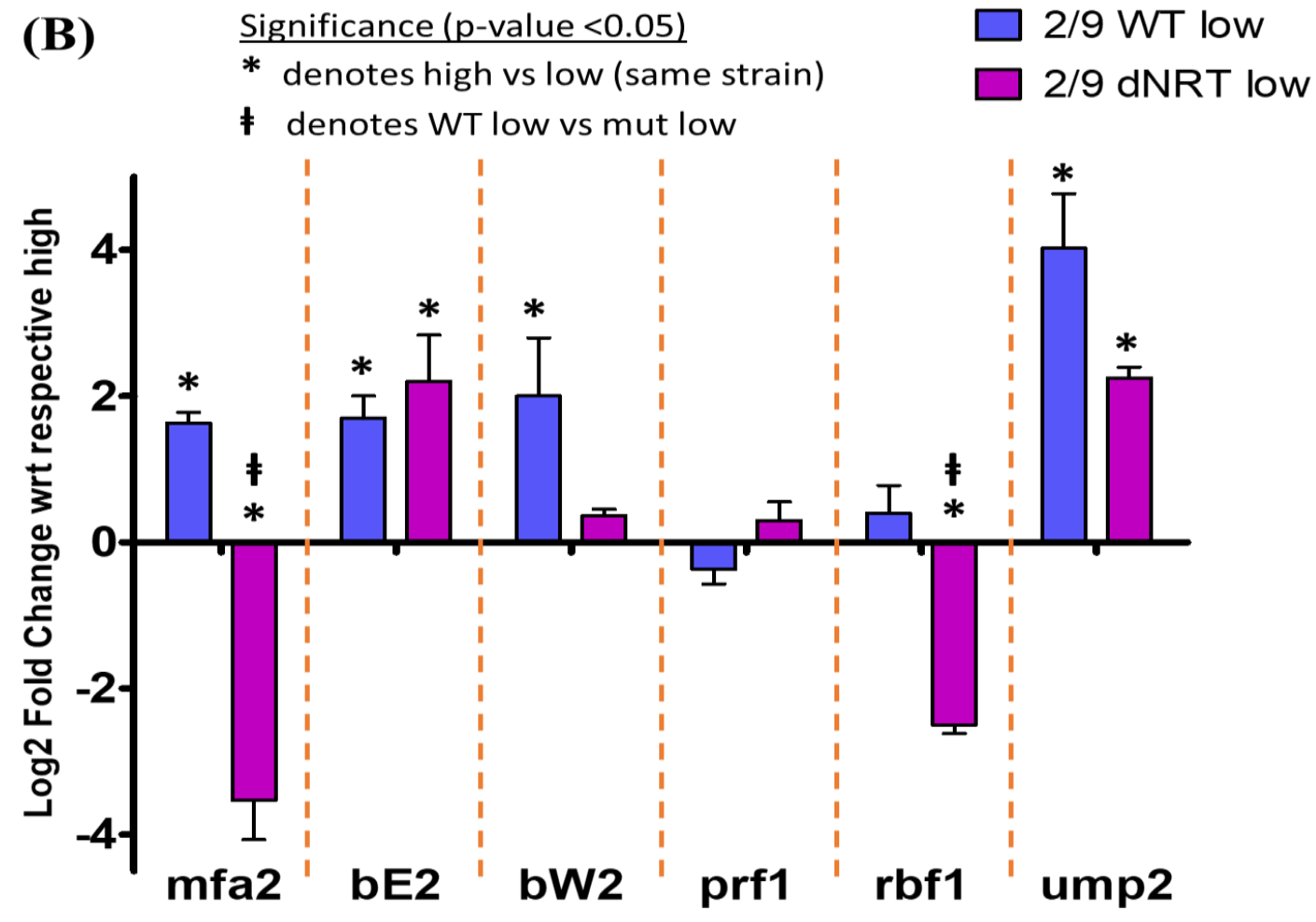

Figure 3. 8 The average log2 fold differences in gene expression by $\Delta$ um03849 mutants grown under low ammonium condition.

The wild type and $\Delta u m 03849$ strains in (A) 1/2 and (B) 2/9 backgrounds were grown under low ammonium and high ammonium conditions. The qRT-PCR was performed on the total RNA extracted from these strains for the mating pathway genes and high affinity ammonium transceptor, Ump2. The $\log 2$ fold changes in expression of the strains grown under low ammonium were normalized against eif 2 and are expressed with respect to (wrt) the same strain grown under high ammonium. Bars represent the averages of biological triplicates and standard errors are indicated in the graphs. The positive values represent increased expression whereas negative values reflect decreased expression. One-way ANOVA followed by Tukey's Multiple Comparison Test was performed in GraphPad Prism. P-value $<0.05$ is considered as significant. * indicates the significant difference in the expression level of target gene for the strain grown on low ammonium with respect to same strain grown on high ammonium. $¥$ indicates the significant difference in the expression level of the mutant on low when compared with wild type on low.

Sequence analysis for the um03849-CRISPR Cas 9 mutants. The $1 / 2$ and 2/9 strains were transformed with vector containing Cas9 and um03848 target (guide RNA). The colonies obtained from transformation were successively streaked in YEPS including 
antibiotic to eliminate the plasmid with Cas9. The elimination of Cas9 plasmid was also confirmed by the absence of Cas9 band when these colonies were screened via PCR using Cas9 specific primers. These selected colonies, which have lost Cas9, were then sequenced in both forward and reverse direction in the um03849 targeted region. The sequences obtained were then aligned along with sequence of wild type strain using MUSCLE (Multiple Sequence Comparison by log-expectation) algorithm ${ }^{153}$ in MEGA$\mathrm{X}$ tool ${ }^{154}$. From the sequencing and alignment with the WT nucleotides (Figure 3.9), it was confirmed that we obtained a 3 bp deletion, 19 bp deletion and 2 bp substitution in the $1 / 2$ mating strain. Similarly, there was a 3 bp deletion and 66 bp insertion in the 2/9 mating strain.

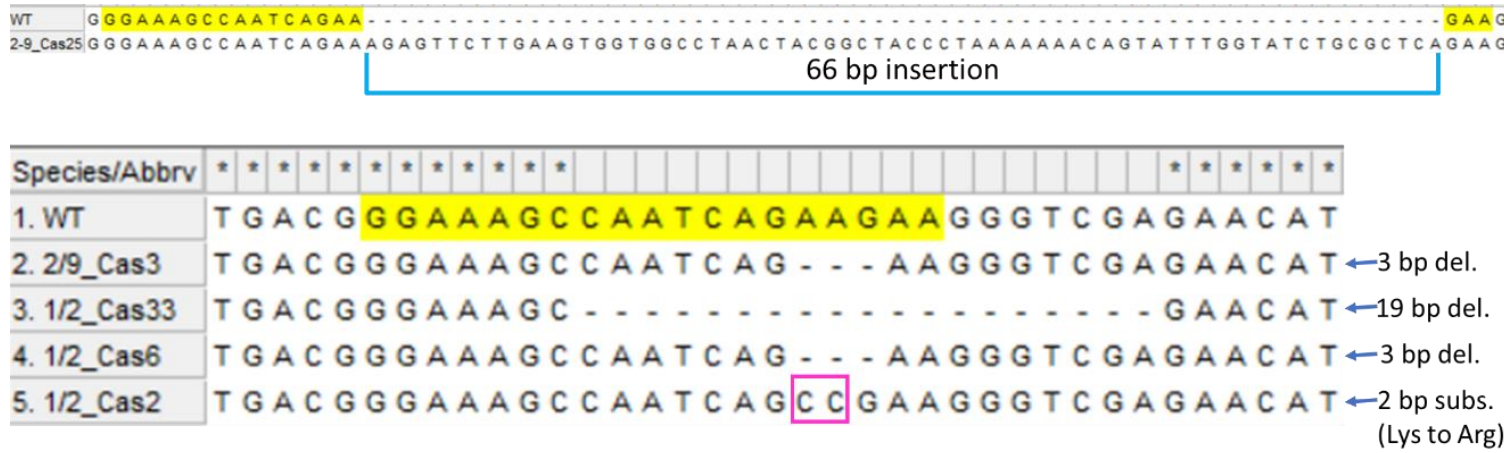

Figure 3. 9 MUSCLE alignment to evaluate INDEL mutation in um03849 gene caused by CRISPR-Cas9 technique.

The CRISPR-Cas 9 mediated INDEL mutation was analyzed by multiple alignment of the DNA sequences along with wild type strain. The yellow highlighted area shows the $20 \mathrm{bp}$ um03849 target region (directed by guide RNA for CRISPR-Cas9 editing technique). The gap shown by dash "-" represents either deletion or insertion of a nucleotide. There was a 66 bp insertion in the 2/9_Cas25 strain. The 3 bp deletion in 2/9_Cas3 and 1/2_Cas6 and 19 bp deletion in 1/2_Cas33 was observed. The pink box on the sequence of 1/2_Cas2 shows the two bases substitution in the target region. This substitution is expected to lead to the change in gene codon, where it is now encoding Arginine instead of coding for Lysine as in wild type. 
The um03849-CRISPR-Cas9 mutants have background specific growth effect when grown in different nitrogen sources media. The identified CRISPR-Cas9 mediated um03849 mutants were 10-fold diluted and spotted on different nitrogen source media. The substitution mutant, 1/2_Cas\#2, the 19 bp deleted mutant, 1/2_Cas\#33, in higher dilutions had reduced growth in all the tested media (Figure 3.10). Moreover, the mutants in $1 / 2$ background showed severely reduced ability to grow in nitrate or nitrite as nitrogen source (Figure 3.10 C, D). No such difference was seen on 2/9 mutants when they were grown in media with different nitrogen sources (Figure 3.10). Specifically, the 2 bp substitution mutant and the 19 bp deleted 1/2 strain had reduced growth in ammonium and urea as a source of nitrogen.

\section{(A) $\underline{\mathrm{AMM}}$}

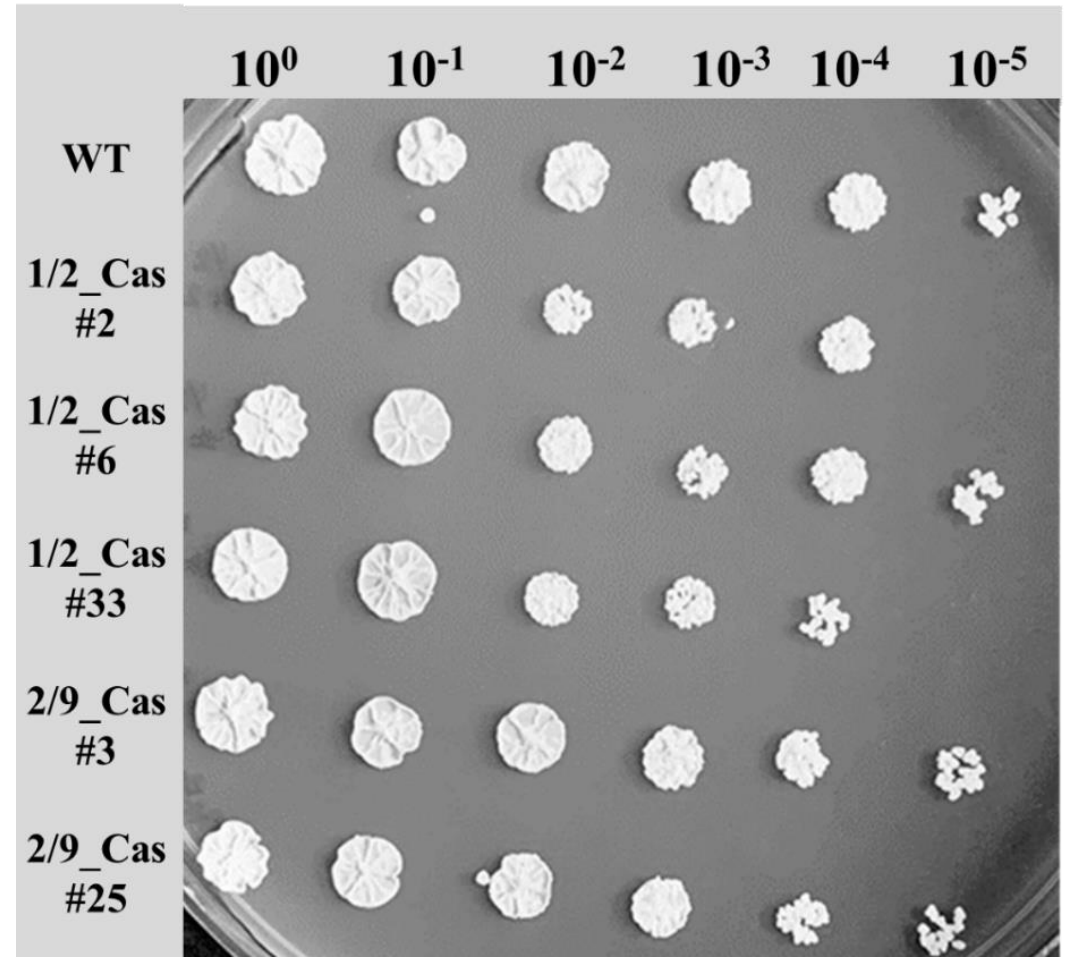


(B) Urea

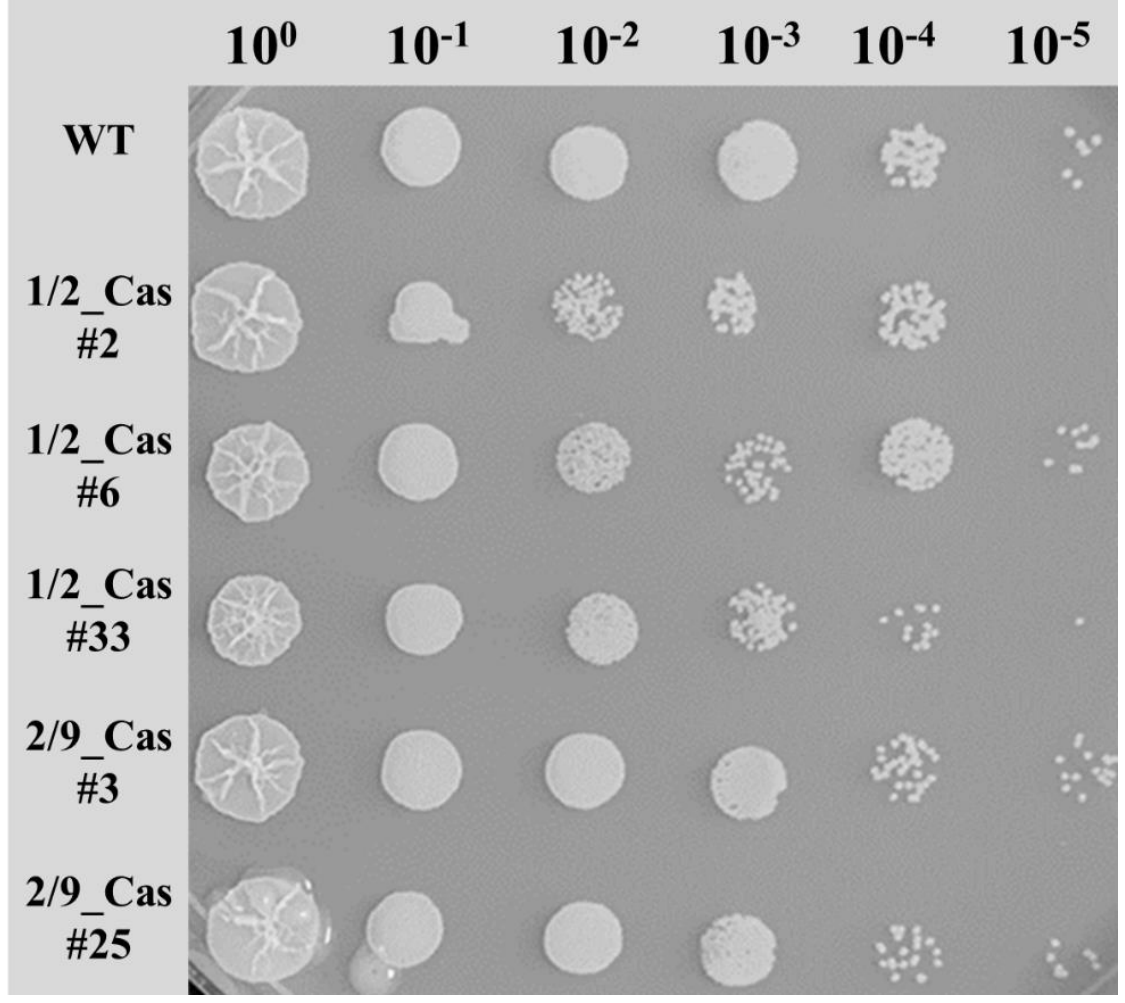

(C) $\underline{\mathrm{NO}}_{3}^{-}$

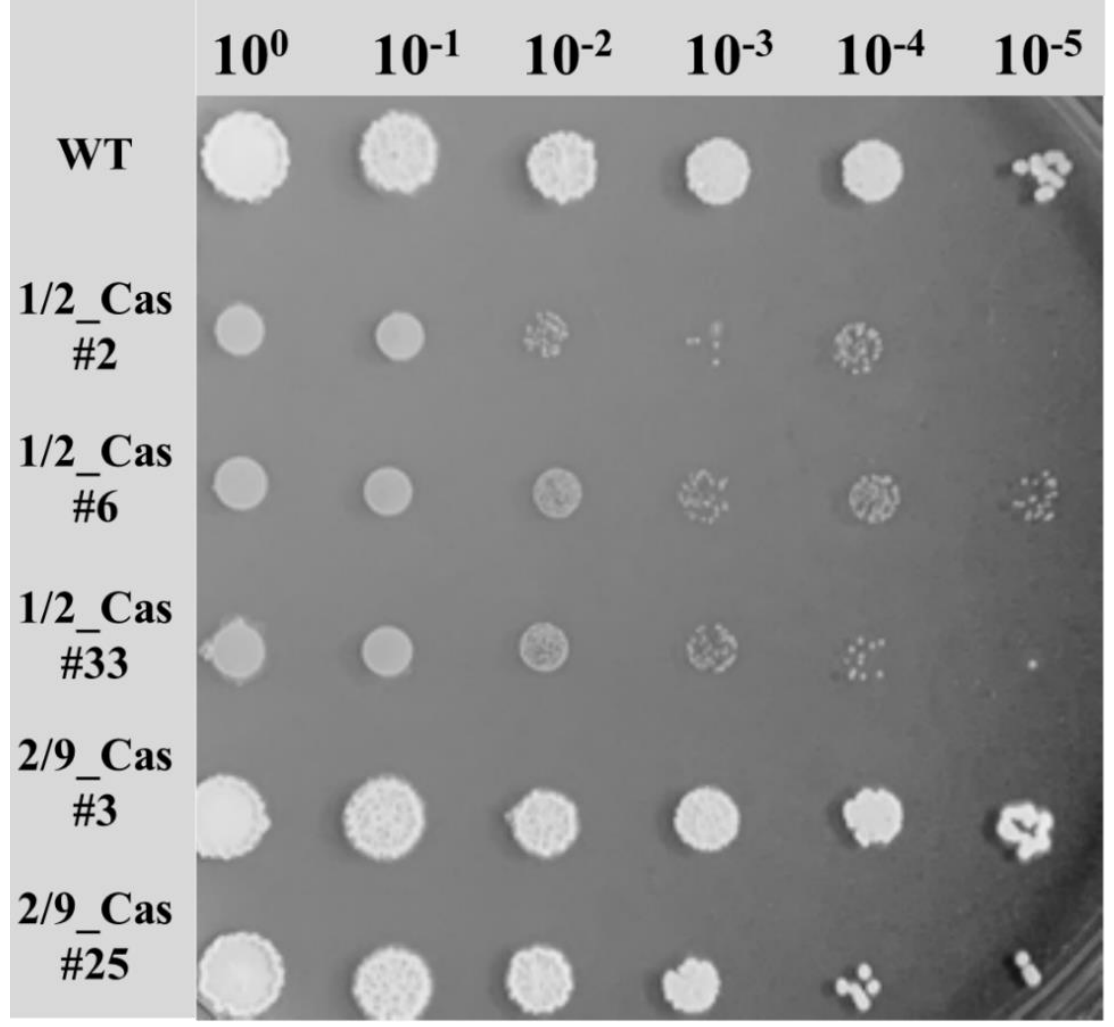




\section{(D) $\underline{\mathrm{NO}}_{2}^{-}$}

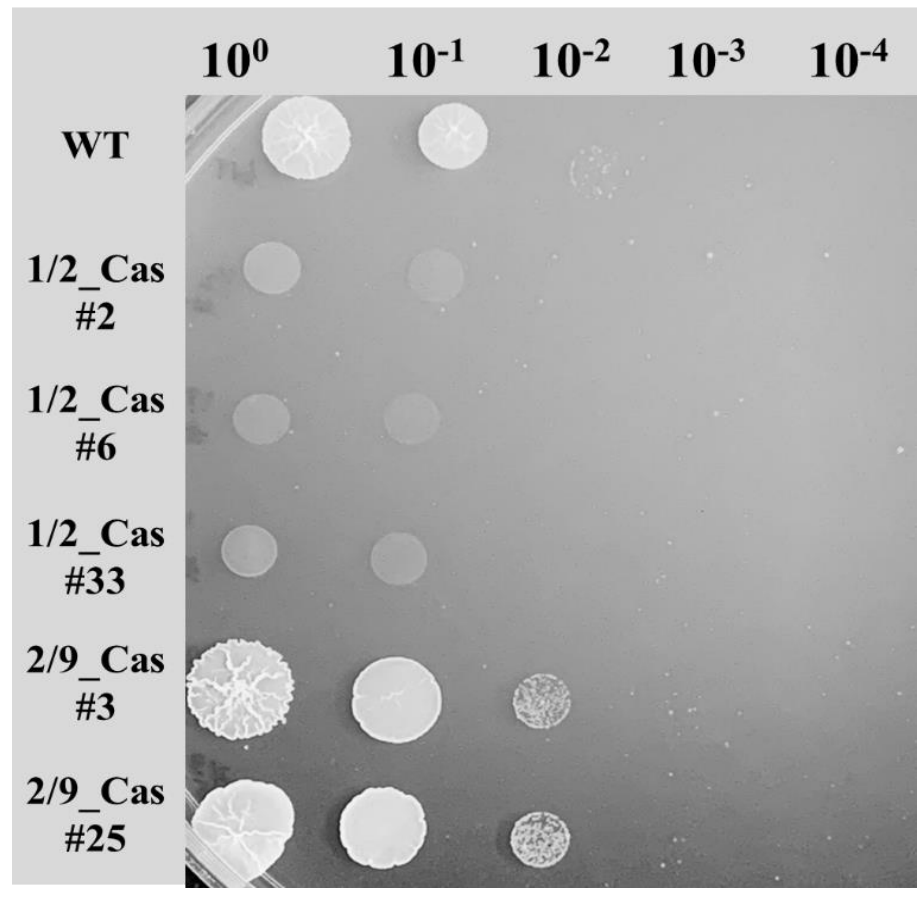

Figure 3. 10 Nitrogen Utilization by CRISPR-Cas 9 mediated um03849 mutants. The 10-fold serial dilutions of overnight grown wild type and um03849 mutant strains were spotted onto minimal media incorporated with (A) $0.3 \%$ w/v Ammonium Sulphate (AMM), (B) $0.3 \% \mathrm{w} / \mathrm{v}$ Urea, (C) $0.3 \% \mathrm{w} / \mathrm{v}$ Potassium Nitrate $\left(\mathrm{NO}_{3}{ }^{-}\right)$and (D) $0.3 \% \mathrm{w} / \mathrm{v}$ Sodium nitrite $\left(\mathrm{NO}_{2}{ }^{-}\right)$Plates were grown at $28{ }^{\circ} \mathrm{C}$ for $48 \mathrm{hrs}$. Growth of mutants were compared with the wild type (WT) where WT here is a $2 / 9$ wild type and the $1 / 2$ wild type also showed similar phenotype as 2/9 (not shown). 1/2_Cas\#2 and 1/2_Cas\#33 had reduced growth in ammonium and urea media. There was a background-specific effect on growth ability of mutants in nitrate and nitrite medium, with only mutants in the $1 / 2$ background displaying the growth defect.

\section{The um03849-CRISPR-Cas9 mutations do not affect the ability of $U$. maydis to mate}

with its opposite partner. The ability of um03849-Cas9 mutants to form dikaryotic aerial hyphae was assessed by co-spotting with the opposite partner on PDA-charcoal medium. The um03849 mutants showed the characteristic white "fuzz" phenotype with its opposite mating partner as well as with the opposite wild type strains similar to that of wild type strains (Figure 3.11). This indicates that the mutation in um03849 doesn't affect the ability of fungus to mate with its compatible partner. 

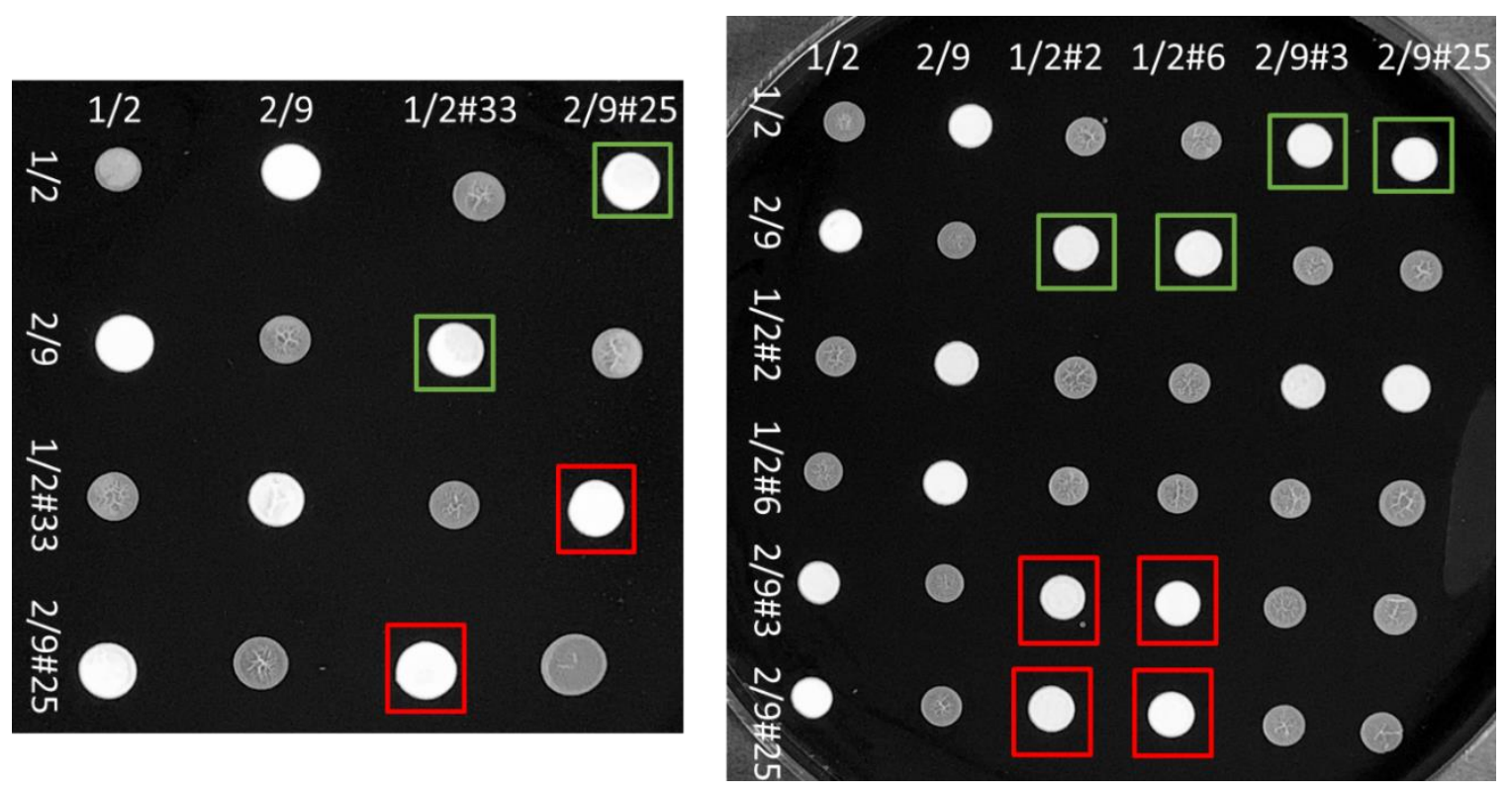

Figure 3. 11 Mating assay for CRISPR-Cas9 mediated um03849 mutants.

Equal mixtures of $1 / 2$ or 2/9 wild type haploid strains or corresponding um03849 mutants were pre-mixed in a tube and co-spotted onto PDA-charcoal plate and grown for $24 \mathrm{hrs}$. A positive mating reaction produced a white "fuzz" phenotype of aerial hyphae production. Red boxes indicate the successful mating of um03849 mutants with compatible mutant partners. Green boxes show the characteristic white "fuzz" phenotype by um03849 mutants with their compatible mating wild type partners. Red boxes represent the "fuzz" phenotype by um03849 with each other. The mutation on um03849 does not affect the ability of $U$. maydis to mate with its compatible partner.

\section{The um03849-CRISPR-Cas9 mutants affect the virulence to the plant based on type}

of mutation. The um03849 mutants were pre-mixed with each other or with compatible wild type and injected into one-week-old maize seedlings. The degree of pathogenicity in the plants infected with mutant strains was compared to that infected with wild type mating strains (1/2 WT X 2/9 WT). In general, the um03849 mutants have reduced virulence to the plants compared to wild type where mutants in $1 / 2$ background have higher effect on pathogenicity than mutants in 2/9 (Figure 3.12). In particular, the mutants with the two base pair substitution, 1/2_Cas\#2, and 19 base pair deletion, 
1/2_Cas\#33, significantly reduced the virulence, as no plants died from infection and fewer than $30 \%$ of plants developed tumors.

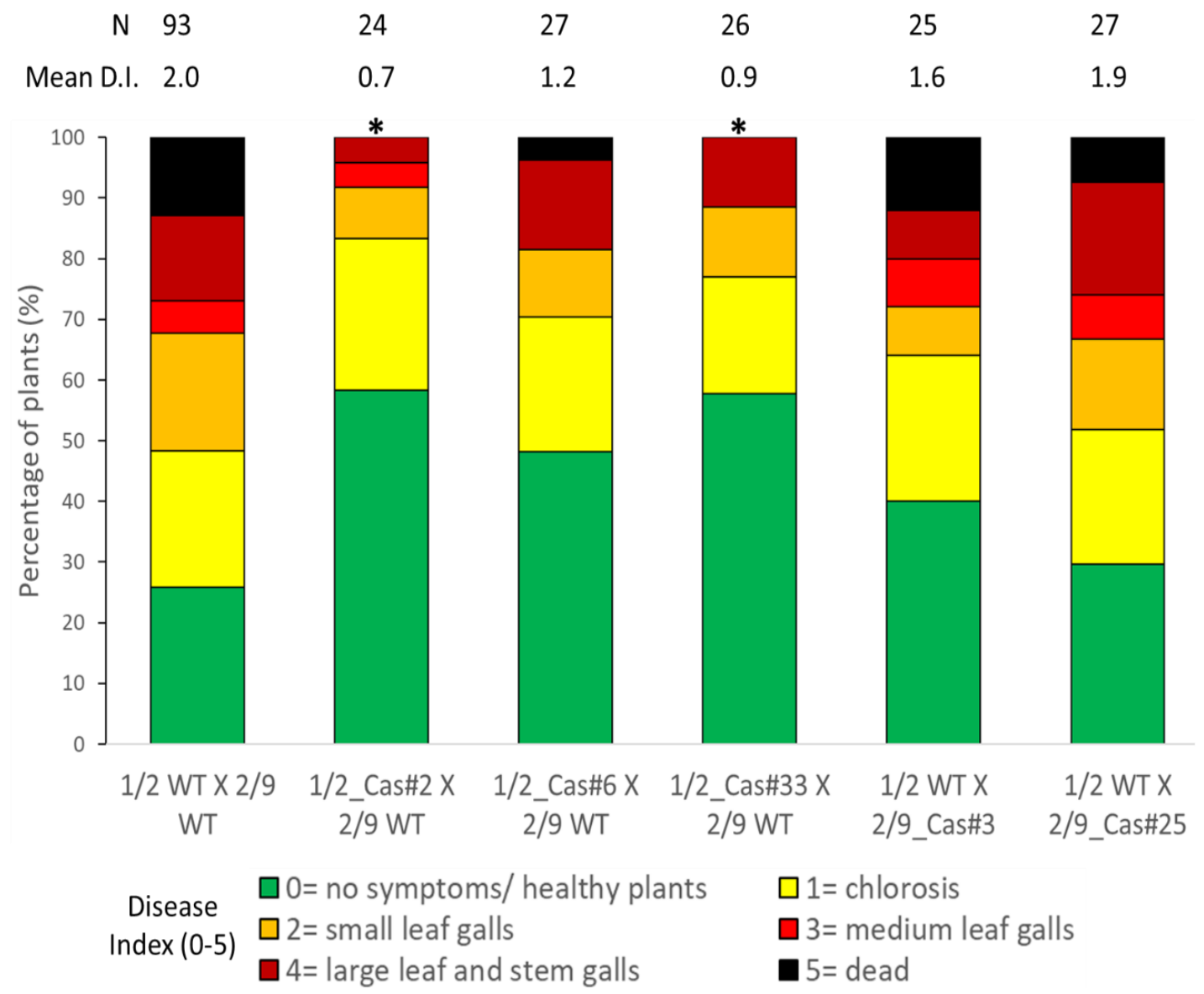

Figure 3. 12 Plant pathogenicity assay for CRISPR-Cas9 mediated um03849 mutants. The indicated strains of $U$. maydis, um03849 mutants combined with opposite wild type strains, were pre-mixed and injected into 7 days old maize seedlings. Disease symptoms were scored 14 days post infection (dpi). The graph displays the percentage of plants with specific symptoms of infection. The $\mathrm{X}$ axis indicates the paired $(1 / 2 \times 2 / 9)$ background that is injected into maize plant. The disease symptom was categorized from 0 to 5 as depicted at the bottom of the figure. Numbers on the top represent the number of plants infected and mean for disease indices (DI). The data were analyzed using Kruskal-Wallis test followed by post-hoc comparison and an asterisk $(*)$ indicates significant difference ( $\mathrm{p}<0.05)$ compared to those where both partners were wild type strains $(1 / 2 X 2 / 9)$. The significant reduction $(\mathrm{p}<0.05)$ of plant pathogenesis was seen in 1/2_Cas\#2 X 2/9 WT and 1/2_Cas\#33 X 2/9 WT. 


\section{Discussion}

The transport proteins in a membrane are very important as they are the first step in a signal-transduction pathway, and they allow cells to communicate with the environment ${ }^{155}$. The nitrate transporter (NRT) is the first gene involved in the assimilation of nitrate. The expression of $n r t$ regulates the expression of the downstream genes involved in nitrate assimilation. And, the presence of a favorable nitrogen source completely inhibits the induction of the nitrate assimilation pathway at the transcriptional level. This is because, $n r t$ is only induced in nitrogen starvation conditions, i.e., in absence of favorable nitrogen source and presence of nitrate in the medium ${ }^{3}$. We observed that the um03849 NRT gene in U. maydis, is significantly upregulated when the wild type strains are grown in low ammonium medium (Figure 3.1), compared to when grown in high ammonium medium. Similarly, Ho et al. 2007 have also shown that the wild type grown in minimal medium or nitrogen starvation medium showed significantly higher expression of the NRT gene. In addition, the transcript analysis in $U$. maydis grown under different nitrogen conditions showed that the ammonium completely repressed the transcript accumulation of NRT, even if nitrate is added to the medium. However, removal of ammonium led to a swift induction of the genes ${ }^{6}$. The fast and strong induction of NRT gene is also observed in the phytopathogenic ascomycetes $F$. fujikoroi, F. oxysporumi, and M. griseae ${ }^{100,156}$.

To study the effect of $U$. maydis NRT gene um03849, we took reverse genetics approaches and created two different types of mutation in the gene. The first approach was utilizing the homologous recombination system of $U$. maydis ${ }^{113}$ which creates a deleted um03849 mutant by replacing a gene with a selective marker gene. The second 
approach was using the robust and currently popular genome editing strategy, Clustered regularly interspaced short palindromic repeats (CRISPR)-Cas9 system ${ }^{157}$ to manipulate the gene um03849 in $U$. maydis. In this approach, a self-replicating plasmid constitutively expressing U. maydis codon-optimized $\operatorname{Cas} 9$ gene and targeted single guide RNA (sgRNA) under control of $U$. maydis U6 polymerase III (snRNA) promoter ${ }^{157}$ was used to induce um03849 targeted gene editing. In a single step transformation with this plasmid vector, sgRNA directed Cas9 endonuclease induces double strand breaks ${ }^{158}$ which are then repaired by error-prone non-homologous end joining (NHEJ) or homologous direct repair pathway, manipulating the defined genomic targets ${ }^{159,160}$. By growing the transformed $U$. maydis in medium without selective antibiotic marker, the fungus loses the self-replicating plasmid to minimize the long-term effects of Cas9. To address the off-target effects of CRISPR-Cas9 system, we employed a high fidelity Cas9 variant on this plasmid, CasHF1, ${ }^{161}$ to manipulate the targeted gene, um03849. From this approach, we were able to obtain different types of INDEL mutations in the gene um03849 in opposite mating partners of $U$. maydis (Figure 3.9). Thus, we obtained um03849 deleted mutants and the targeted gene edited mutants of um03849 in U. maydis and thereby assessed the role of um03849 gene in different aspects of fungal lifecycle.

When compared to wild type, the haploid filamentation of $\triangle u m 03849$ on low ammonium, did not differ, both having characteristic filaments on the surface of colonies. The deletion of the high affinity ammonium transceptor, Ump2, resulted in the loss of filamentation when grown on low ammonium medium ${ }^{79,82}$. The STRING database analysis showed the protein-protein interaction between nitrate and ammonium transporters in U. maydis (Figure 5.1 in Chapter V). However, the filamentation 
phenotype shown by deletion of two transporters were not the same. Ump2 directly senses the low ammonium condition and filaments help to forage during nitrogen starvation $^{79}$.

The reduced growth of $\Delta u m 03849$ (Figure 3.3) and 1/2 CRISPR-Cas9 mutated strains (Figure 3.10) on nitrate medium compared to wild type indicated that the NRT affects the transport and utilization of nitrate. From the study of growth curves in $U$. maydis grown on nitrate medium in Chapter II, we have observed that the 2/9 wild type can grow faster in the nitrate medium than 1/2 wild type strain (Figure 2.6 C, D). Similarly, in this study, we saw that the Cas9-mutated 2/9 strain showed less effect of the mutations than did mutants of the $1 / 2$ strain (Figure $3.10 \mathrm{C}$ ). The growth of $100 \mathrm{X}$ diluted wild type haploids was completely inhibited on nitrite medium (Figure 3.10 D). This indicates the nitrite is an even less preferred source of nitrogen than nitrate to the fungus. The extracellular nitrite induces only a slight or a significantly delayed expression of nitrate assimilatory genes in $F$. fujikuroi ${ }^{100}$. Because of delayed gene expression, to keep the intracellular nitrite concentrations below toxic levels, the fungus will only import nitrite when it is absolutely necessary for growth ${ }^{162}$.

The deletion of um03849 decreased growth in Congo Red as compared to wild type (Figure $3.4 \mathrm{C}$ ), and similar to decreased growth observed in ump2 deleted mutants in Congo Red ${ }^{82}$. In addition to being an osmotic stress, response to sorbitol reflects cell wall integrity ${ }^{163}$. We observed that the deletion of um03849 impaired growth in medium with sorbitol (Figure 3.4 D). Several components are efficiently organized at and in the cell membrane to maintain the cell structure and stability in different environments. The secondary transport proteins are composed of variable number of transmembrane $\alpha$ - 
helical segments connected by intra- and extracellular loops ${ }^{155}$. Therefore, in addition to roles in transport and signal transduction, membrane transporter proteins provide a specific regulation and stability of membrane structure. Thus, the deletion of a transmembrane protein, e.g., nitrate transporter um03849 (this study) or ammonium transporter Ump2 ${ }^{82}$ affects the growth of $U$. maydis in cellular stressors such as Congo Red and sorbitol.

One of the plant defense systems against microbes is the oxidative burst constituting the production of reactive oxidative species (ROS), superoxide and $\mathrm{H}_{2} \mathrm{O}_{2}$, at the site of infection ${ }^{164}$. Therefore, plant pathogens must develop a mechanism to detoxify an oxidative burst. Membrane transporters play an important role in cellular resistance to oxidative stress in the phytopathogenic fungus Alternaria alternata ${ }^{165}$. There is a link between the antioxidant capacity and haloterance of fungi, suggesting that if a fungus has oxidative stress tolerance, then it can also tolerate a high saline environment and vice versa ${ }^{166}$. For this reason, we tested the growth ability of NRT deleted mutant, $\Delta u m 03849$, in oxidative and saline media. We observed that $\Delta u m 03849$ mutants grew like their respective wild type progenitors in media with $\mathrm{NaCl}$ (saline) or $\mathrm{H}_{2} \mathrm{O}_{2}$ (oxidative) (Figure $3.4 \mathrm{~A}, \mathrm{~B}$ ).

The deletion of um03849 in U. maydis reduced its virulence in infected maize (Figure 3.6). The ability of a fungus to respond to the changing environment and nutritional conditions is necessary to establish infection in its host plant. Although nitrate is not a preferred source of nitrogen, it is important for the fungus to be able to utilize nitrate because it is major nitrogen source used in fertilizers and very prevalent in croplands ${ }^{167}$. The form of nitrogen available in a plant determines the nitrogen supply to 
the fungus and further determines the disease severity on the plant ${ }^{168}$. Nitrate assimilation predominantly takes place in leaves in young maize plants ${ }^{169}$ and $U$. maydis infects aerial parts, mostly leaves. Tumors produced in maize plants after infection with U. maydis rely on the import of organic nitrogen from noninfected, systemic source leaves ${ }^{95}$. The deletion of nit2, a regulator of nitrogen metabolic repression (NMR), in $U$. maydis strongly decreased virulence ${ }^{6}$. Similarly, many phytopathogenic fungi show reduced virulence upon deletion of NMR regulatory genes ${ }^{34,35,170}$. These results indicate that the unfavored nitrogen sources also can regulate the virulence of fungi. When we crossed $\Delta u m 03849$ mutants with compatible wild type strains, we observed that the infection capability was drastically reduced in the 1/2 WT X 2/9 $\Delta$ um03849 group. However, the infection by $1 / 2 \Delta u m 03849$ X $2 / 9$ WT was slightly reduced with only $20 \%$ of infected plants showing tumors, out of which only $8 \%$ had large tumors (Figure 3.6). During the biotrophic stage of fungus infection, amino acids are readily available to the fungus at the site of infection but are limiting growth in the early necrotrophic stage ${ }^{6}$. The high nitrogen availability increases susceptibility of plants to biotrophs, while it decreases susceptibility of plants to necrotrophs ${ }^{171}$.

The CRISPR-Cas9 mediated mutation in um03849 also affected the ability of $U$. maydis to infect the plants but only by the strains that had amino acid substitution or a frameshift mutation (Figure 3.12). A frameshift mutation is a type of mutation in which there has been insertion or deletion of bases that are not multiples of three, whereas an inframe mutation is an insertion or deletion of three or a multiple of three bases. For this reason, the former, as its name suggests, alters the reading frame during translation and causes fewer changes in the encoded protein downstream of the insertion. In this study, 
for the $1 / 2$ background, we obtained a frameshift mutant, via 19 bp deletion; the second mutant had a 2 bp substitution. When these strains were crossed with 2/9 WT and used to infect plants, they exhibited significantly reduced virulence to plants (i.e., none of the infected plants died, only about $24 \%$ of plants developed tumors, and half of those had only small size tumors). On the other hand, the remaining CRISPR-Cas9 mediated mutants did not display reduced pathogenicity. This result is consistent with the growth of these um03849 mutants on different nitrogen source media (Figure 3.10): frameshift mutants had reduced growth (evident at the highest dilutions), whereas in-frame mutations didn't display this defect. The in-frame CRISPR-Cas9 mediated mutation of bE/bW in SG200 retains the fungal characteristic "fuzz" $\left(\mathrm{Fuz}^{+}\right)$phenotype on charcoal medium whereas the frameshift mutation showed Fuz phenotype ${ }^{157}$. The 2 bp substitution mutant in 1/2 background (1/2_Cas\#2, Figure 3.9) occurred by the replacement of ' $T T^{\prime}$ ' at the $47^{\text {th }}$ and $48^{\text {th }}$ nucleotide sequence in um03849 by 'GG', leading to substitution of arginine instead of lysine in the encoded protein (amino acid translation via Expasy translate tool: https://web.expasy.org/translate/). Arginine (Arg) and lysine (Lys) are basic amino acids containing guanidinium and $\mathcal{E}$-amino group, respectively. Because of these groups, they are positively charged under physiological conditions. Although both amino acids function as basic residues, the arginine with its guanidinium group allows electrostatic interactions in three possible directions whereas lysine can only form one direction interaction. This provides arginine with stronger interaction forming a more stable protein architecture than lysine ${ }^{172}$. The GFP (Green Fluorescent Protein) variants created by mutating the maximum number of lysine residues on the surface of the protein to arginine relatively increases the stability of 
variants to urea, alkaline $\mathrm{pH}$, and ionic detergents ${ }^{173}$. The stability of arginine residues to chemical denaturants is due to the electrostatic interaction by guanidium group in arginine, confirmed by geometric properties ${ }^{172,174}$. On the other hand, the altered electrostatic interactions induced by mutagenesis of surface lysine to arginine adversely affected the protein folding and decreased the productivity of the functional form of the protein ${ }^{173}$. The single ionic interaction in lysine makes it flexible and prone to post translational modifications which might develop the differential sense of cellular metabolic state and response to nutritional or environmental changes ${ }^{175}$. The study of an ionizable amino acid residues in the internal positions in staphylococcal nuclease (SNase) showed the large shift in $\mathrm{pK}_{\mathrm{a}}$ values for Lys residue in different $\mathrm{pH}$ conditions ${ }^{176}$ whereas Arg at the same internal position exhibit constant $\mathrm{pK}_{\mathrm{a}}$ value. Internal arginine residues also play critical roles in enzyme active site ${ }^{177,178}$ and in a variety of transport channels ${ }^{179}$. However, a L36R variant in SNase lacked an enzymatic activity without affecting the protein fold ${ }^{180}$. Thus, it is hard to interpret the complex regulation of amino acids lysine and arginine in stability and function of um03849 protein. However, we observed that the mutation of lysine to arginine in um03849 reduced the pathogenicity to the plants.

We observed that either a deletion or functional mutation of the um03849 gene reduced the pathogenicity of the fungus to plants. However, there was no effect on the mating of the fungus with its compatible partners. To understand the role of um03849 in regulation of virulence to plants, we performed qRT-PCR to study expression of mating targets (Figure 3.8). All the mating targets were downregulated in $\triangle 4 m 03849$ mutants on low ammonium, compared to WT grown in same conditions. The significant 
downregulation of $m f a 2$ and $r b f 1$ genes might have played a role in reduced virulence by 2/9 $\Delta u m 03849$ but these genes were significantly downregulated in $1 / 2 \Delta u m 03849$ as well. The severe repression of $r b f 1$ for the 2/9 $\Delta u m 03849$ might be the possible explanation for the strong effect as Rbf1 is important for pathogenic development. However, further study of the expression of genes involved in pathogenic development inside the host plant may provide additional insights for the observed phenotypes. Also, constructing um03849 complementation on $\Delta u m 03849$ strains will be helpful and necessary for clearer conclusions. The fusion of compatible mating partners occurs by the mating type specific lipoprotein pheromones, encoded by $m f a$ gene, that are secreted ${ }^{181}$. These pheromones are perceived by the cells of opposite mating type by pheromone binding transmembrane receptors (Pra1/2) and both cells stop budding and start formation of conjugation tubes ${ }^{182,183}$. Therefore, pheromone stimulation has a vital role on the conjugation tube formation and mating in $U$. maydis. In our study, although we observed the significant downregulation of $m f a l / 2$ genes in $\Delta u m 03849$ mutants, the ability of mutant to mate (shown by characteristic "fuzz" in Figure 3.5) was unaffected. This might be the low ammonium condition required for mating was not same on charcoal medium as it would be in environment. Therefore, the measurement of expression of $\mathrm{pral} / 2$ encoding pheromone receptors, as well as expression of $\mathrm{mfal} / 2$ (normally up-regulated in the presence of mating partner) will be helpful to understand this apparent contradiction.

Once compatible fungal cells have fused, the fate of the resulting dikaryon depends on the products of the $b$ mating type locus. Only cells with different $b$ alleles can successfully form a dikaryon ${ }^{86}$. The $\mathrm{bE}$ and $\mathrm{bW}$ homeodomain proteins encoded by the 
$b$ locus dimerize to form a functional $\mathrm{bE} / \mathrm{bW}$ heterodimer. The $\mathrm{bE} / \mathrm{bW}$ complex then binds to conserved DNA motifs upstream of $b$ responsive genes to regulate the $b$ dependent filament formation and pathogenic development ${ }^{61,127}$. Since all the subsequent steps in development of the dikaryon occur inside the host, the $b$ locus can be considered as the molecular switch from the saprophytic to the biotrophic stage of the fungus ${ }^{76}$. One of the genes that is directly regulated by $\mathrm{bE} / \mathrm{bW}$ heterodimer is the transcription factor Rbf1, which is master regulator of the series of genes facilitating sexual and pathogenic development of $U$. maydis ${ }^{138}$. In our study, we observed that reduction of $b E$ and/or $b W$ levels significantly downregulates the expression of rbfl (Figure 3.8). The overexpression of $u m p 2$ rescued the filamentation defect of $b$ locus deletion ${ }^{82}$. The $u m p 2$ on deleted mutants grown on low ammonium was significantly upregulated compared to their respective mutants grown on high ammonium but significantly downregulated compared to WT grown on low ammonium conditions (Figure 3.8). However, the interaction between $u m 03849$ and $u m p 2$ can be further investigated by overexpression of ump2 in $\Delta u m 03849$ mutants and vice versa. Additionally, constructing um03849 complementation strains will be helpful to better understand the role of this gene on infection.

In summary, we observed that the nitrate transporter gene um03849 in U. maydis is induced under low ammonium or nitrogen starvation conditions. The nitrate transporter is able to sense nitrate in the environment as shown by reduced growth of the $\Delta u m 03849$ mutants on nitrate medium. This indicates that Um03849 is important for nitrate assimilation in $U$. maydis. In addition, um03849 is also involved somehow in activation of virulence-related genes. These observations open new research questions as to the 
specific phase of pathogenic development where Um03849 plays a role and what other regulatory proteins such as Nmr (nitrogen metabolic regulation) proteins are involved in this process. 


\section{CHAPTER IV}

\section{NITRATE ASSIMILATORY GENES, UM03848 AND UM03849, IN MATING AND PATHOGENICITY IN USTILAGO MAYDIS}

\section{Introduction}

The biotrophic fungus Ustilago maydis is able to use nitrate as a sole source of nitrogen ${ }^{3,184}$. For the utilization of nitrate in the fungi, nitrate transport is the first step and it induces the expression of the other genes related to its assimilation. Then nitrate is reduced to nitrite and to ammonium by nitrate reductase and nitrite reductase, respectively. In $U$. maydis, um03849, um03847 and um03848 have been designated as a potential genes for nitrate transporter, nitrate reductase and nitrite reductase, respectively $47,55,56$. Even though nitrate reductase (NAR), whose gene is now known as $u m 03847^{55}$, in $U$. maydis was studied (as a narl) ${ }^{56}$, there has not been much investigation for the nitrate transporter (NRT) or nitrite reductase (NiR).

In this study, we characterize the role of NRT and NiR genes in $U$. maydis in terms of fungal mating ability and pathogenesis. Previously we have observed that the deletion of either NRT, um03849, (Chapter III) or NiR, um03848, (Chapter II) alone reduces the pathogenesis to the host plant, the degree to which was dependent on the specific fungal genetic background. In this chapter, we study the effects of double deletion of both um03848 and um03849 on characteristics of growth, mating, and 
pathogenicity of the fungus. The mutant strain with the double deletion, $\Delta u m 03848$ Aum03849, was examined for phenotypes such as ability to grow in different media, haploid/dikaryotic filamentation formation and virulence. The haploid $\Delta u m 03848$ Dum03849 mutants showed slightly more extensive filamentous growth when cultivated in low ammonium medium for 4 days, compared to their respective wild type progenitors. When $\Delta u m 03848 \Delta u m 03849$ mutants were grown in different nitrogen source media, the strains showed less ability to use urea or nitrate as a source of nitrogen. Similarly, the mutants, along with the respective wild type strains, were grown in different stress media such as $\mathrm{NaCl}$, sorbitol and Congo red. The $\Delta u m 03848 \Delta u m 03849$ mutants showed reduced ability to grow in such stressor media. In examining mating efficiency, $\Delta u m 03848 \Delta u m 03849$ was unable to show the characteristic "fuzz" phenotype when co-spotted with opposite mating partners in the charcoal medium, indicating an inability to mate. In addition, $\Delta u m 03848 \Delta u m 03849$ strains showed reduced virulence to host maize plants in a manner specific to a particular genetic background/progenitor strain. Hence, we observed that the deletion of both NRT and NiR in U. maydis made the fungus vulnerable to stress conditions and reduces the virulence to the host plant. However, the reduction in virulence due to the double deletion was specific to the mating background of the fungus.

\section{Methods}

Strains and growth conditions. The $U$. maydis strains used in this study are listed in Table 4.1. Escherichia coli strain DH5 $\alpha$ (Thermo Fisher) was used for all cloning and plasmid maintenance. U. maydis strains were grown at $27{ }^{\circ} \mathrm{C}$ on solid or in liquid YEPS, 
PDA media (Brachmann, König, Julius, \& Feldbrügge, 2004) or Holliday Salt media (Holliday, 1974). Strains grown in liquid media were grown overnight shaking at 250 rpm. E. coli cells were grown in LB medium supplemented with $200 \mu \mathrm{g} / \mathrm{mL}$ ampicillin at $37^{\circ} \mathrm{C}$.

Table 4. 1 Strains used in Chapter IV

\begin{tabular}{lll}
\hline Ustilago Strain & Genotype & Reference \\
\hline $1 / 2$ & $a l b 1$ & Gold et al., 1997 \\
$2 / 9$ & $a 2 b 2$ & Gold et al., 1997 \\
$1 / 2 \Delta u m 03849-H y g R$ & $a l b 1$ um03849::hyg$g^{R}$ & This study \\
$\Delta u m 03848-C b x R$ & $\Delta u m 03848:: c b x R$ & \\
$2 / 9 \Delta u m 03849-H y g R$ & $a 2 b 2$ um03849::hyg$g^{R}$ & This study \\
$\Delta u m 03848-C b x R$ & $\Delta u m 03848:: c b x R$ & \\
\hline Plasmid & Genotype & Reference \\
\hline pum03849-HygR & up - hyg $g^{R}-$ down $a m p^{R}$ Ori & This study \\
\hline
\end{tabular}

Primer design and PCR. Primers listed in Table 3.2 in Chapter III were used to create deletion constructs in this Chapter. Primers were designed and PCR reactions were performed as described previously (Chapter II).

Vector construction and Genetic manipulation. The deletion of um03849 in U. maydis $\Delta u m 03848-C b x R$ strains were obtained by homologous recombination ${ }^{113}$. Deletion construct of um03849 was obtained as described previously in Chapter III by PCR amplification of flank regions, hygromycin resistance cassette and ampicillin resistance plus Ori cassette, followed by the Gibson overlap-technique ${ }^{117}$ to obtain a vector designated as pum03849-HygR, which was then transformed into commercially-provided competent E. coli DH5 $\alpha$ (Thermo Fisher). DNA sequencing (Eurofins, Louisville, KY) confirmed structure of the pum03849-HygR vector; this was used as template with 
primers flanking the desired construct to amplify it $\left(u m 03849:: H y g^{R}\right.$ construct) as a linear piece. The construct was then transformed by the polyethylene glycol/ protoplast method 113,116 into $U$. maydis strains of both mating-type backgrounds of $\triangle u m 03848-C b x R$. In this way, the um03849 ORF was replaced with the hygromycin resistance cassette in strains already deleted for um03848, creating double deletion mutants for genes um03848 and um03849. Transformed U. maydis cells were selected on YEPS agar plates containing $150 \mu \mathrm{g} / \mathrm{ml}$ hygromycin B (Gold Biotech). The successful transformation of $\Delta u m 03848 \Delta u m 03849$ was confirmed by PCR and by loss of gene transcription, as evidenced by Real-time quantitative PCR (qRT-PCR) on cDNA, using the primers that would distinguish the transformants from the respective wild type strains (Figure A.11).

Cell growth, nitrogen utilization, stress tests, mating and pathogenicity. Cell growth rates, utilization of different nitrogen sources, cell wall stress tests, mating assays and plant infections were performed as described previously (Chapter II).

\section{Results}

\section{Double deletion of um03848 (NiR) and um03849 (NRT) influences filamentation in}

low ammonium media. The filamentation phenotype was observed for wild type and $\Delta u m 03848 \Delta u m 03849$ strains of the $1 / 2$ and 2/9 backgrounds. All strains were streaked onto low ammonium $\left(30 \mu \mathrm{M} \mathrm{NH}_{4}\right)$ medium and incubated for 4 days at $28{ }^{\circ} \mathrm{C}$. The individual colonies were observed under a compound microscope at 100X magnification. When filamentation in $\Delta u m 03848 \Delta u m 03849$ haploid strains were compared to their respective wild types, 2/9 $\Delta u m 03848 \Delta u m 03849$ showed thinner and more branched filaments (Figure 4.1 A). To quantify the differences in filamentation, the ratio of the 
filaments to the central colony area was calculated by using a 2 X 2 square grid on photos obtained from microscope. In the grid, we manually counted the number of squares covered by filaments and the number of squares covered by central cell mass and ratio was taken. While 2/9 $\Delta u m 03848 \Delta u m 03849$ showed slightly higher filamentation ratio compared to $2 / 9 \mathrm{WT}$, the $1 / 2 \Delta u m 03848 \Delta u m 03849$ displayed significantly higher filamentation than 1/2 WT (Figure $4.1 \mathrm{~B}$ ).

\section{(A)}
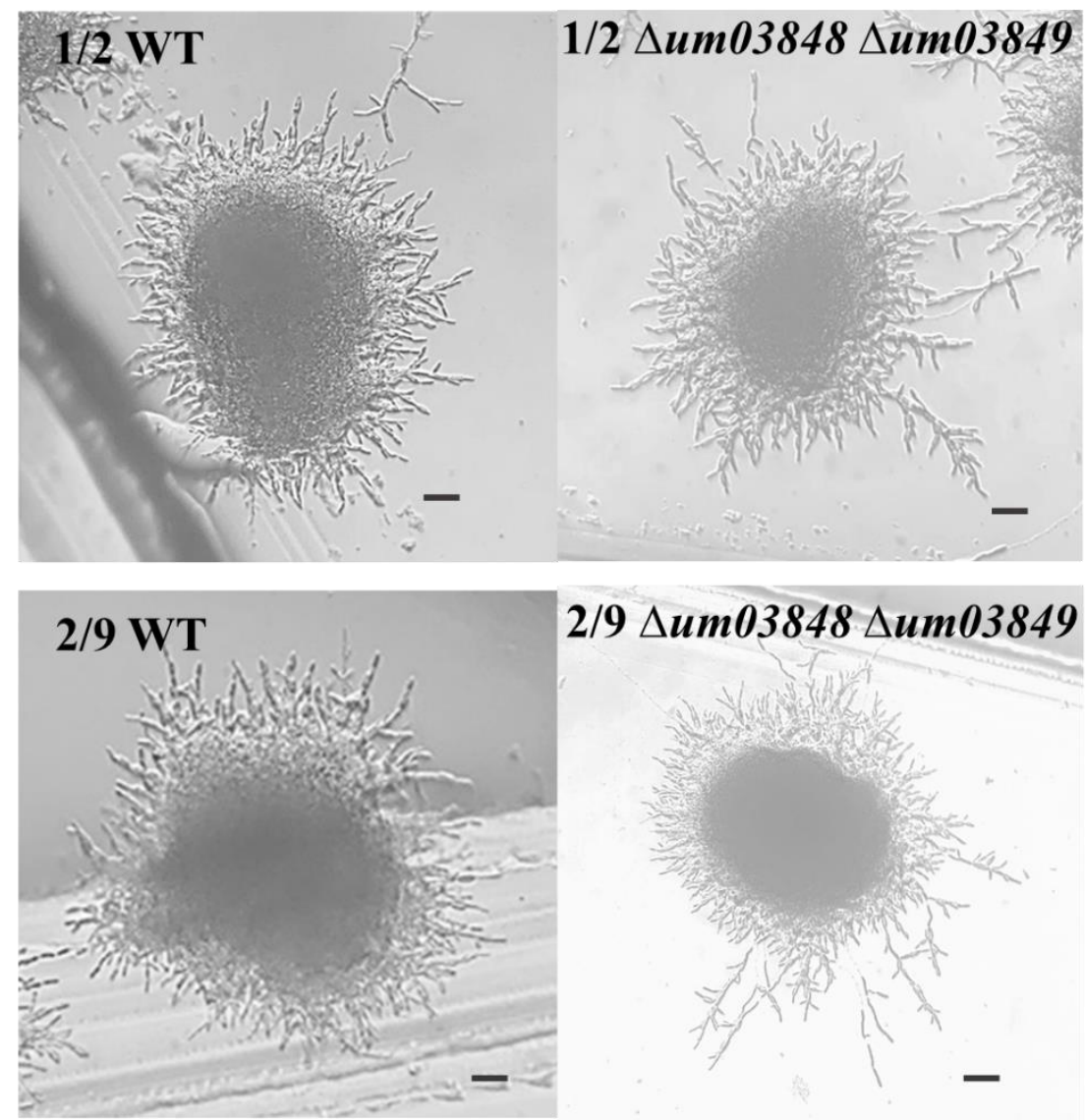

2/9 $\Delta u m 03848 \Delta u m 03849$

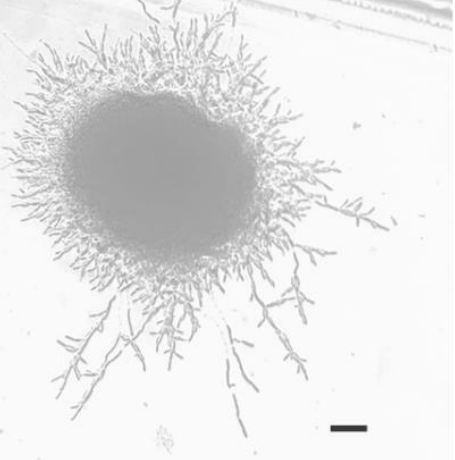




\section{(B)}

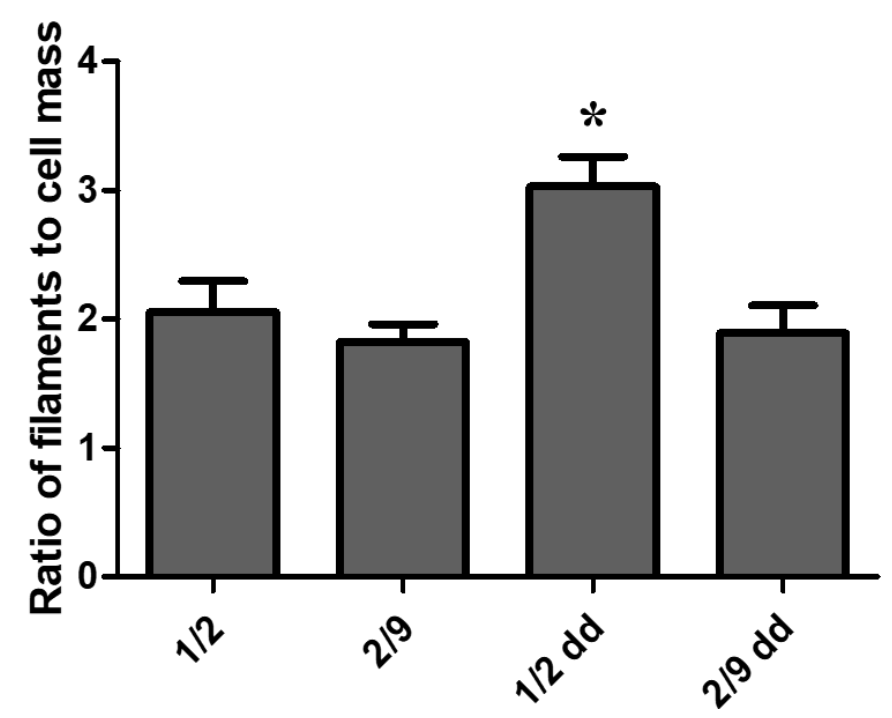

Figure 4. 1 Phenotype observation for $\Delta$ um03848 $\Delta$ um03849 mutants grown on low ammonium medium.

(A) Filamentation assay where haploid cells (labeled) were grown on low ammonium medium for 4 days. Size bars, $100 \mu \mathrm{m}$. (B) Characterization of filamentation by the ratio of filaments to central colony area. The average of at least three colonies from three different plates is shown in the graph. Student's t-test was performed in mutant strains comparing it to the respective wild type strains. The asterisk $(*)$ indicates significant difference $(\mathrm{p}<0.05)$ in $1 / 2 \Delta u m 03848 \Delta u m 03849(1 / 2 \mathrm{dd})$ compared to $1 / 2$ wild type.

\section{The double deletion of $u m 03848$ and $u m 03849$ affects the growth of fungus in}

different nitrogen media. When um03848 and um03849 double deletion mutants were grown in a medium with ammonium or no nitrogen provided, they were able to grow in similar ways as their respective wild type strains (Figure 4.2 A, D). However, when the strains were grown in complex nitrogen sources such as urea and nitrate, growth of mutants was affected (Figure 4.2 B, C). 


\section{(A) $\underline{\text { AMM }}$}

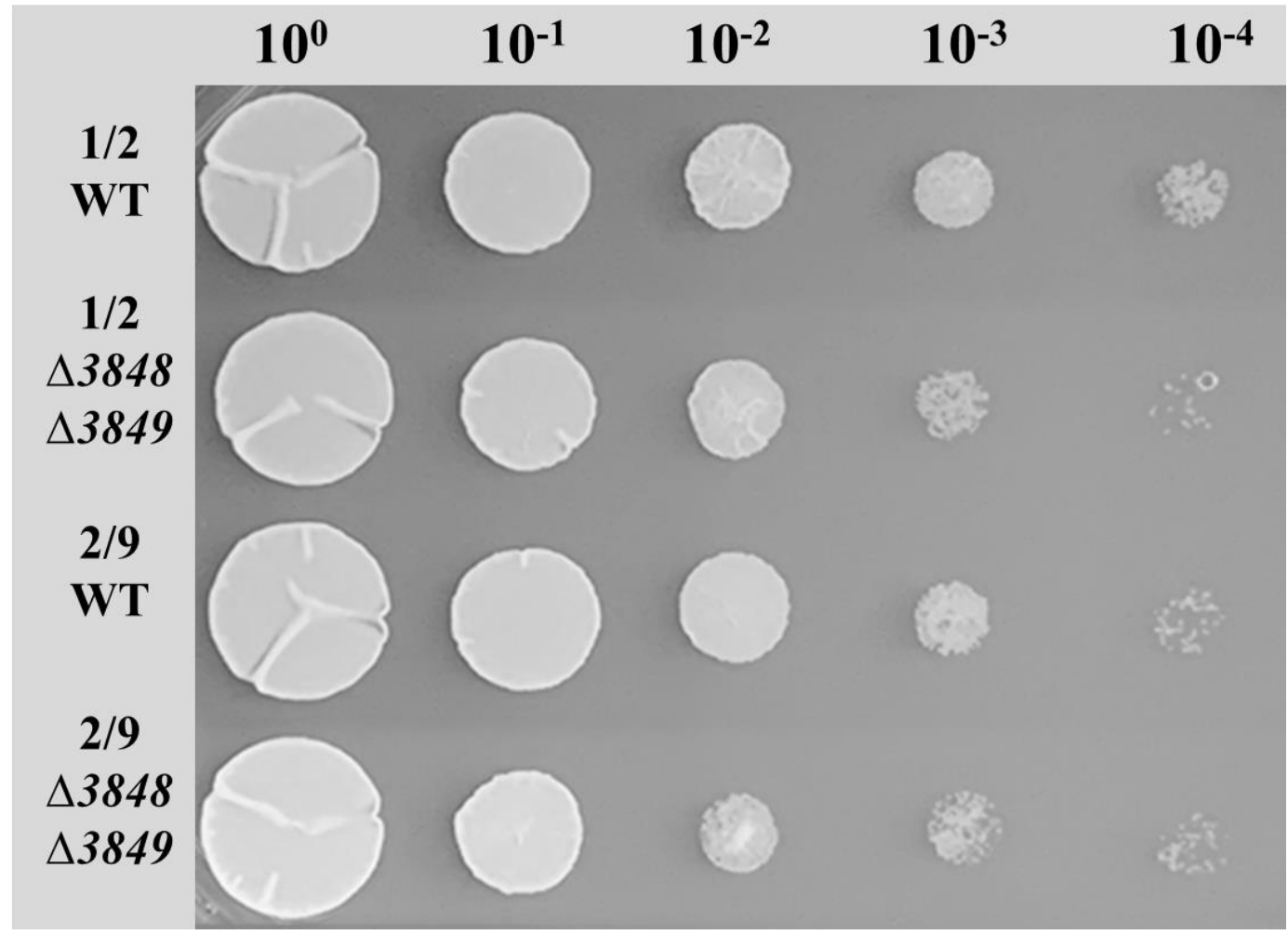

(B) Urea

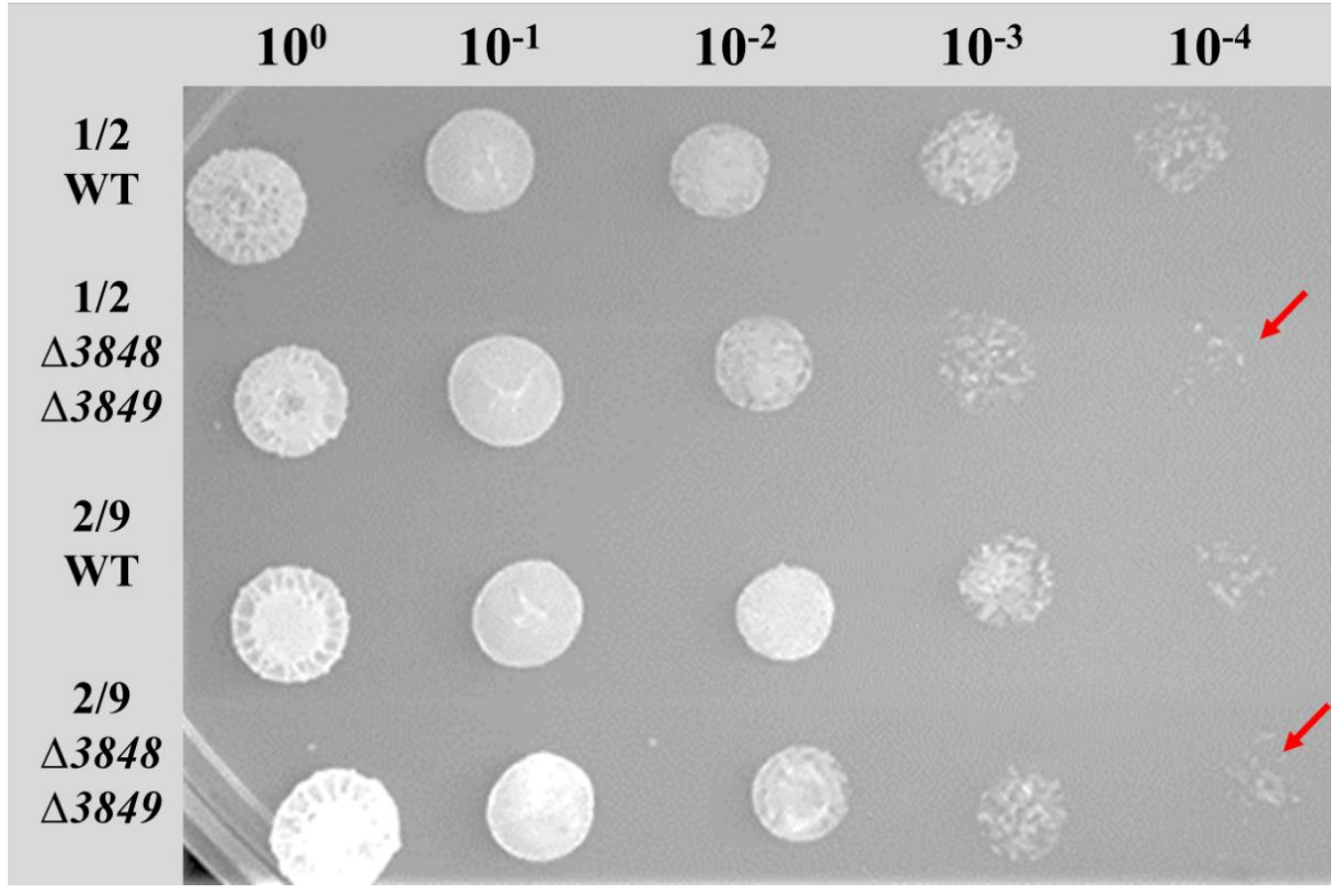




\section{(C) $\underline{\mathrm{NO}}_{3}^{-}$}

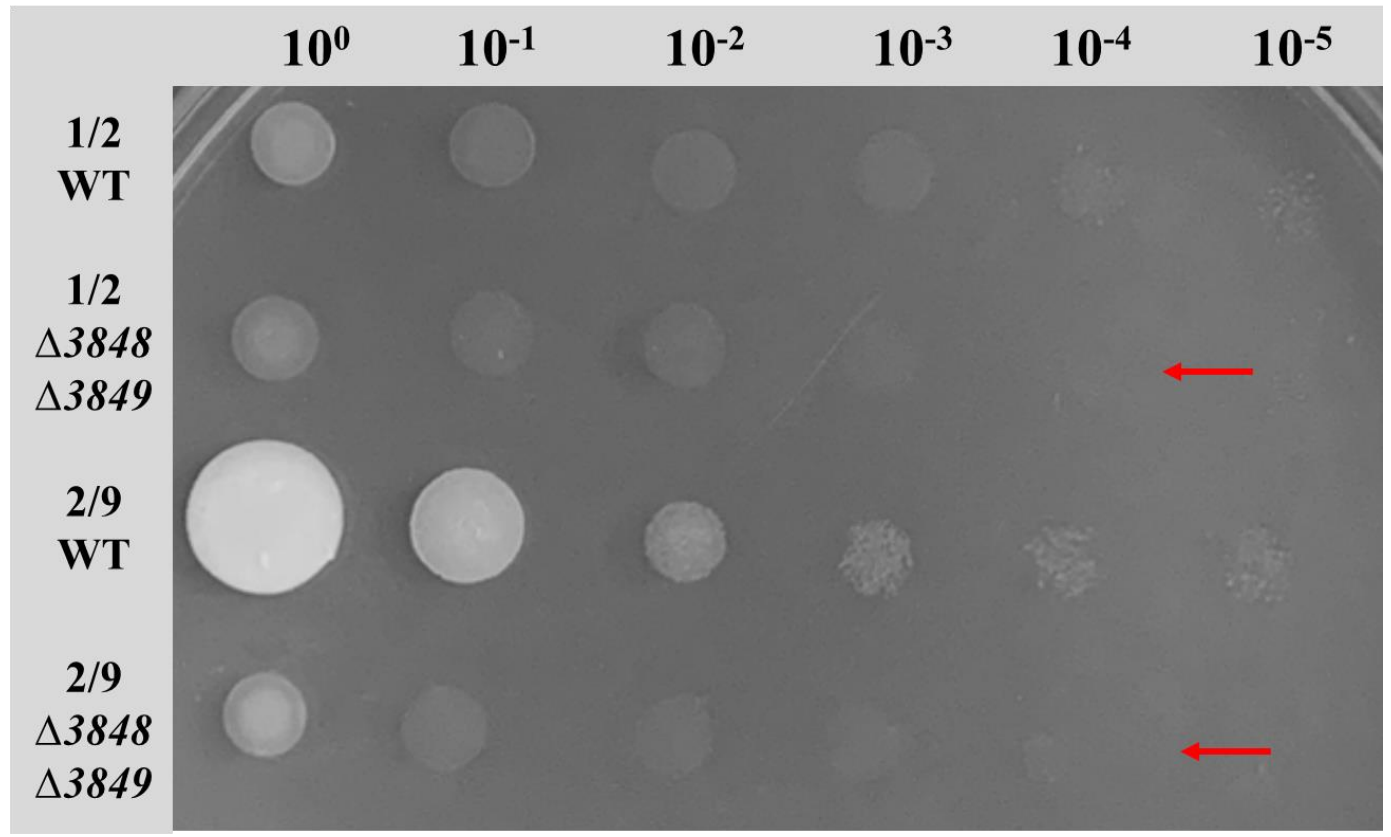

(D) $\underline{-\mathrm{N}}$

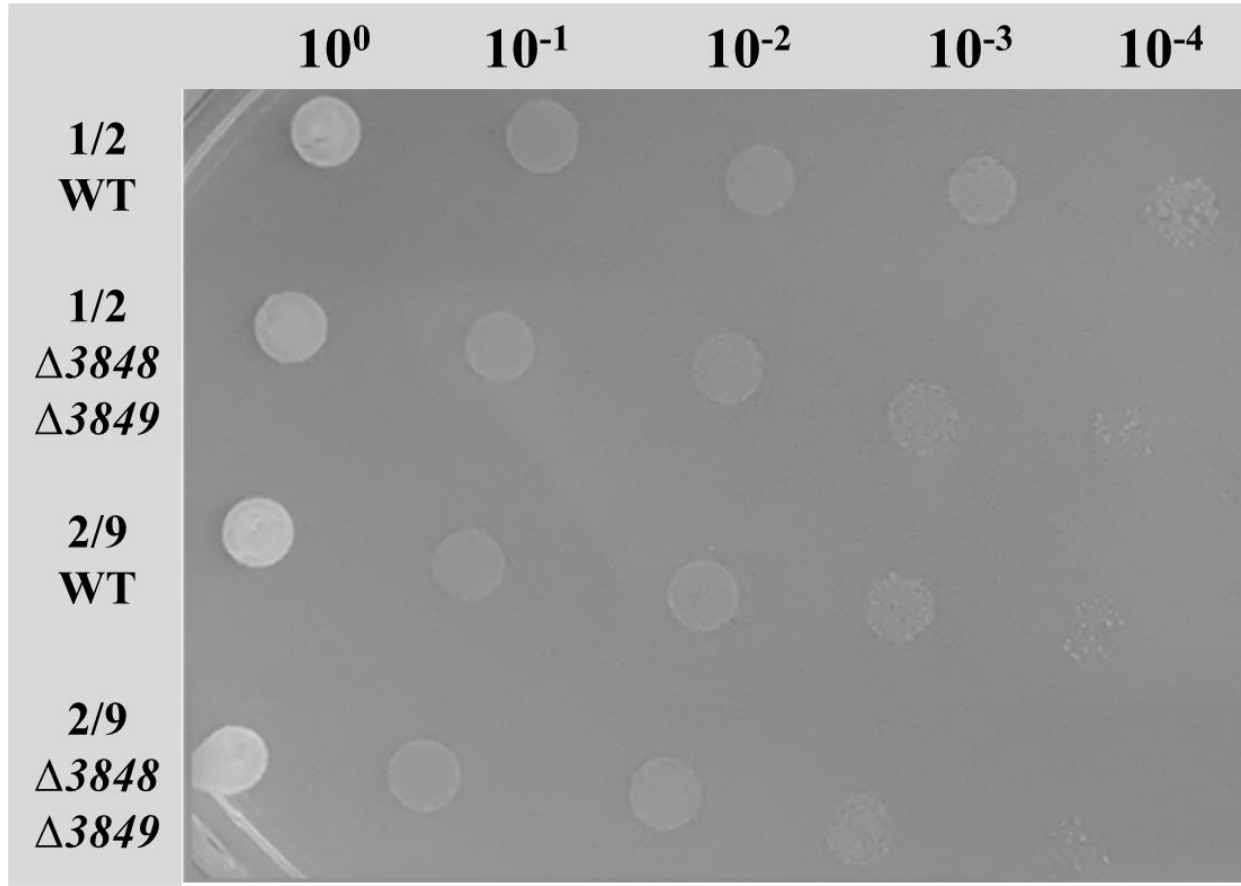

Figure 4. 2 Nitrogen utilization by double deletion mutants.

10 -fold serial dilutions of wild type and $\Delta u m 03848 \Delta u m 03849$ strains were spotted onto minimal media incorporated with (A) $0.3 \% \mathrm{w} / \mathrm{v}(23 \mathrm{mM})$ Ammonium Sulphate (AMM), (B) $0.3 \% \mathrm{w} / \mathrm{v}(50 \mathrm{mM})$ Urea, (C) $0.3 \% \mathrm{w} / \mathrm{v}(30 \mathrm{mM})$ Potassium Nitrate $\left(\mathrm{NO}_{3}{ }^{-}\right)$and (D) 
no nitrogen (-N). Plates were grown at $28{ }^{\circ} \mathrm{C}$ for $48 \mathrm{hrs}$. Mutants were compared with their respective wild type progenitor strains. Red arrows show the limited effect on the growth of mutants.

\section{The double deletion of $u m 03848$ and $u m 03849$ affects the growth of fungus in}

different stress media. The um03848 and um03849 double deletion mutants were spotted onto YEPS agar supplemented with $1 \mathrm{M}$ sorbitol or $1 \mathrm{M} \mathrm{NaCl}$ as high osmotic media and with $1 \mathrm{M}$ Congo red (CR) as cellular stress medium affecting cell wall. The growth ability for the $\Delta u m 03848 \Delta u m 03849$ mutants was reduced in all the stress media, especially more obvious at higher cell dilutions (Figure 4.3).

\section{(A) $\underline{\text { Sorbitol }}$}

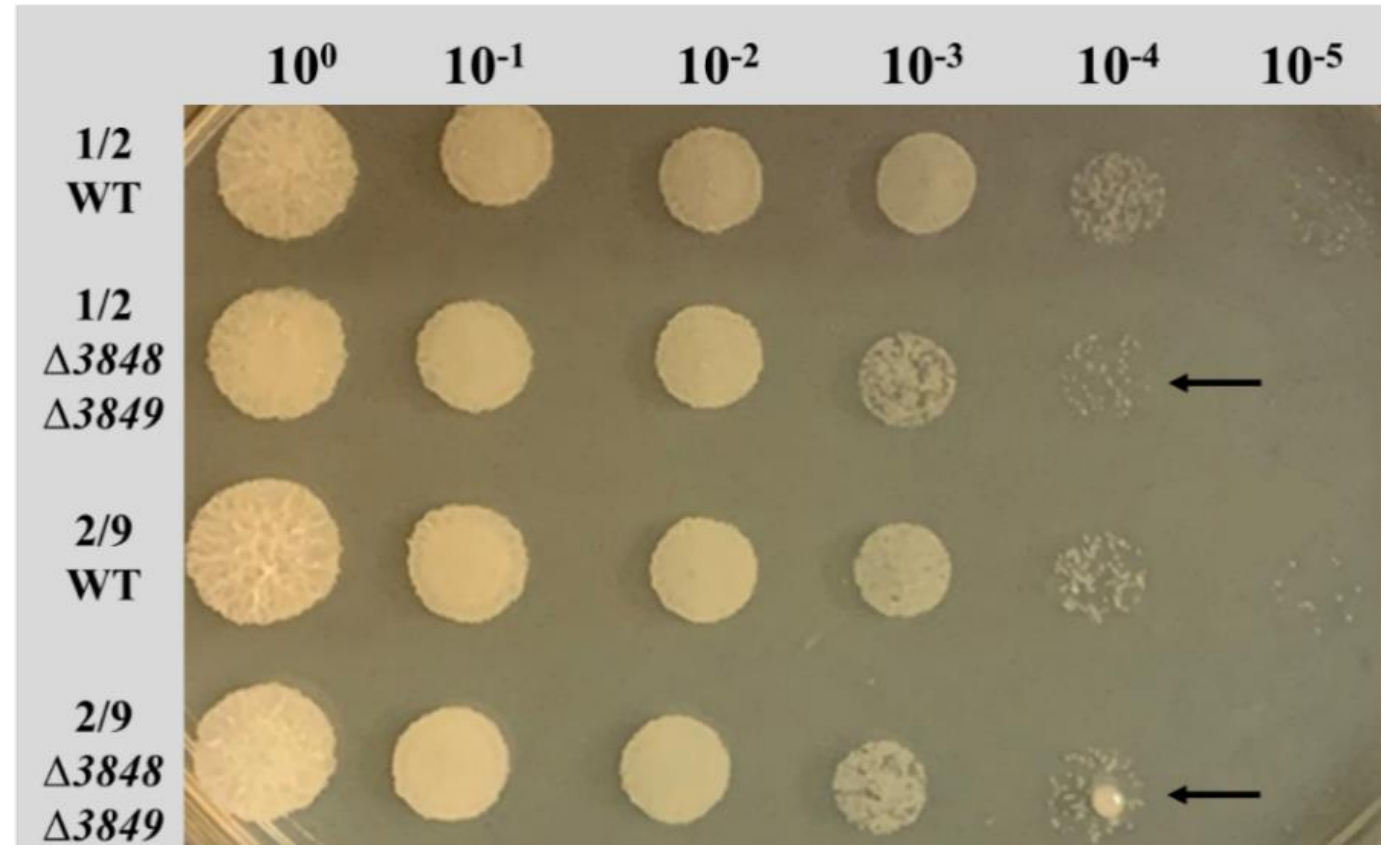


(B) $\underline{\mathrm{NaCl}}$

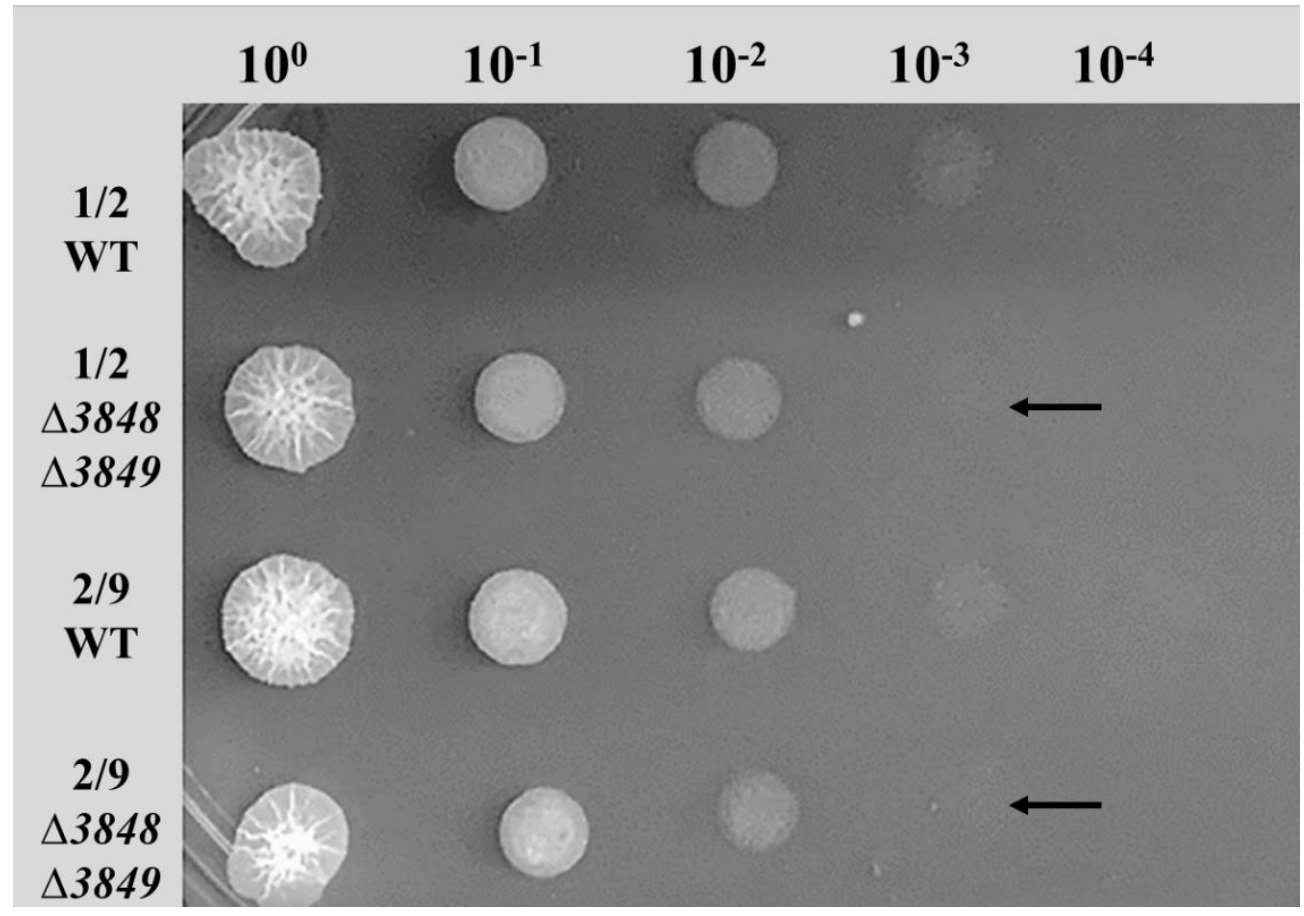

\section{(C) $\underline{\text { CR }}$}

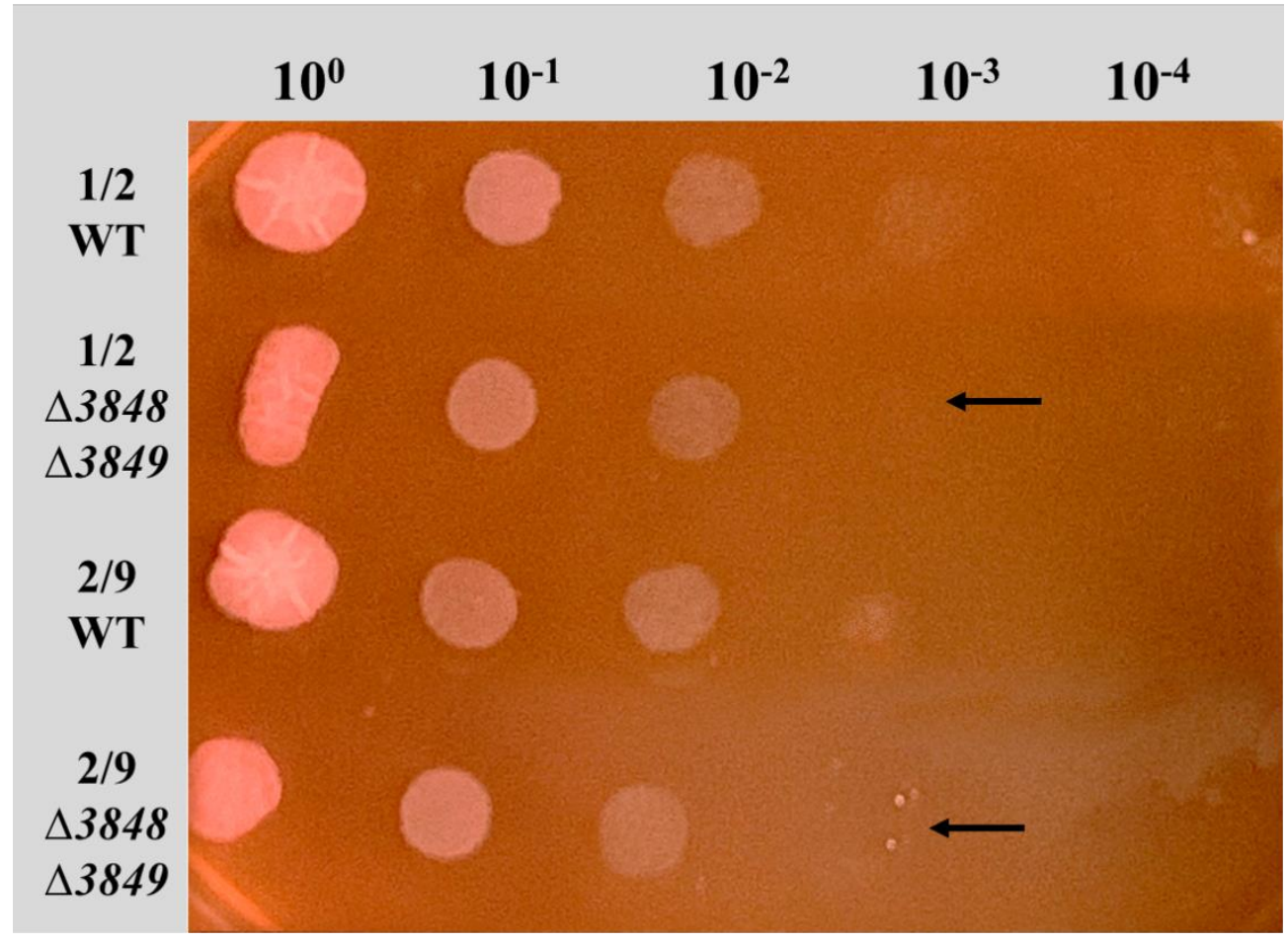

Figure 4. 3 Growth of double deletion mutants in stress media.

The 10-fold serial dilutions of wild type and $\Delta u m 03848 \Delta u m 03849$ strains were spotted onto YEPS agar supplemented with (A) $1 \mathrm{M}$ sorbitol, or (B) $1 \mathrm{M} \mathrm{NaCl}$ or (C) $1 \mathrm{mM}$ 
Congo red (CR). $\mathrm{NaCl}$ and sorbitol are osmotic stressors and Congo red is a cell wall stressor. Black arrows show the effect of double deletion in all the stress media tested.

Double deletion of $u m 03848$ and $u m 03849$ reduces mating ability. Equal mixtures of haploid strains were pre-mixed in a tube and spotted onto PDA containing $1 \%$ charcoal. After $24 \mathrm{~h}$ of incubation, the formation of white "fuzz" was observed to indicate the degree of successful mating for both wild type mating and those involving strains bearing the $\Delta u m 03848 \Delta u m 03849$ lesions. The mutants were unable to mate with partners also bearing the double deletion (Figure 4.4); this was also true when wild type 1/2 was mated with the mutant 2/9 $\Delta u m 03848 \Delta u m 03849$. In contrast, mating of $1 / 2 \Delta u m 03848$ sum03849 with wild type, 2/9 showed the white "fuzz" phenotype (Figure 4.4).

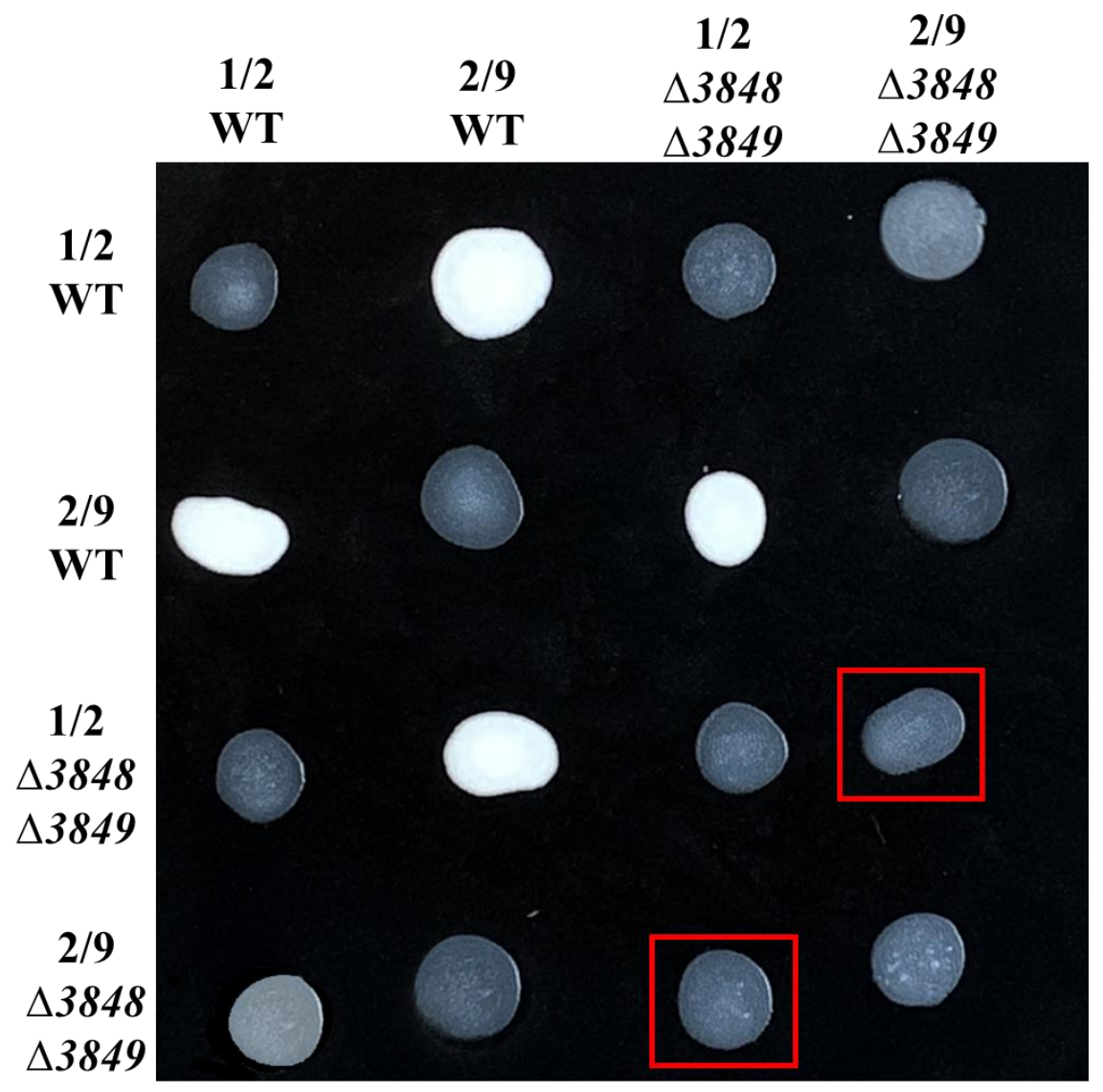

Figure 4. 4 Mating assay of $\Delta$ um03848 $\Delta$ um03849 on PDA-charcoal medium. 
Plate mating assay on $1 \%$ charcoal incorporated PDA plate for $1 / 2$ and 2/9 wild type and corresponding um03848 um03849 deletion mutants. Equal mixtures of haploid strains were pre-mixed in a tube and spotted onto the PDA plate and grown for $24 \mathrm{hrs}$ at $28{ }^{\circ} \mathrm{C}$. A positive mating reaction produced a white "fuzz" phenotype of aerial hyphae production. Red boxes in figure indicate the $\Delta u m 03848 \Delta u m 03849$ (1/2 X 2/9) not able to mate or absence of white "fuzz" phenotype. This same phenotype is seen for matings between $1 / 2$ wild type and 2/9 $\Delta u m 03848 \Delta u m 03849$

\section{Double deletion of $u m 03848$ and um03849 reduces pathogenesis in a manner}

dependent on the genetic background of the mutant. Compatible opposite mating strains of double deleted mutants were pre-mixed and injected into one-week-old maize seedlings to determine ability to infect plants and elicit disease symptoms. To assess whether the functional copies of genes um03848 and um03849 are required in both partners for infection or not, the double deleted mutants were combined with the opposite mating-type wild type strain and pathogenicity was also studied for these pairings. The mated $\Delta u m 03848 \Delta u m 03849$ mutants showed a significant reduction in virulence to the plant when compared to the virulence shown by wild type partners (Figure 4.5). The 1/2 WT X 2/9 $\Delta u m 03848 \Delta u m 03849$ also showed a significant reduction in the virulence, in a similar way as that shown by $\Delta u m 03848 \Delta u m 03849(1 / 2 X 2 / 9)$. However, $1 / 2$ $\Delta u m 03848 \Delta u m 03849 \times 2 / 9 \mathrm{WT}$ did not display a significant reduction in virulence on the host (Figure 4.5). These results indicated that the $U$. maydis NRT and NiR double deletion significantly reduced virulence when present in the 2/9 background strain, but not in the $1 / 2$ background. 


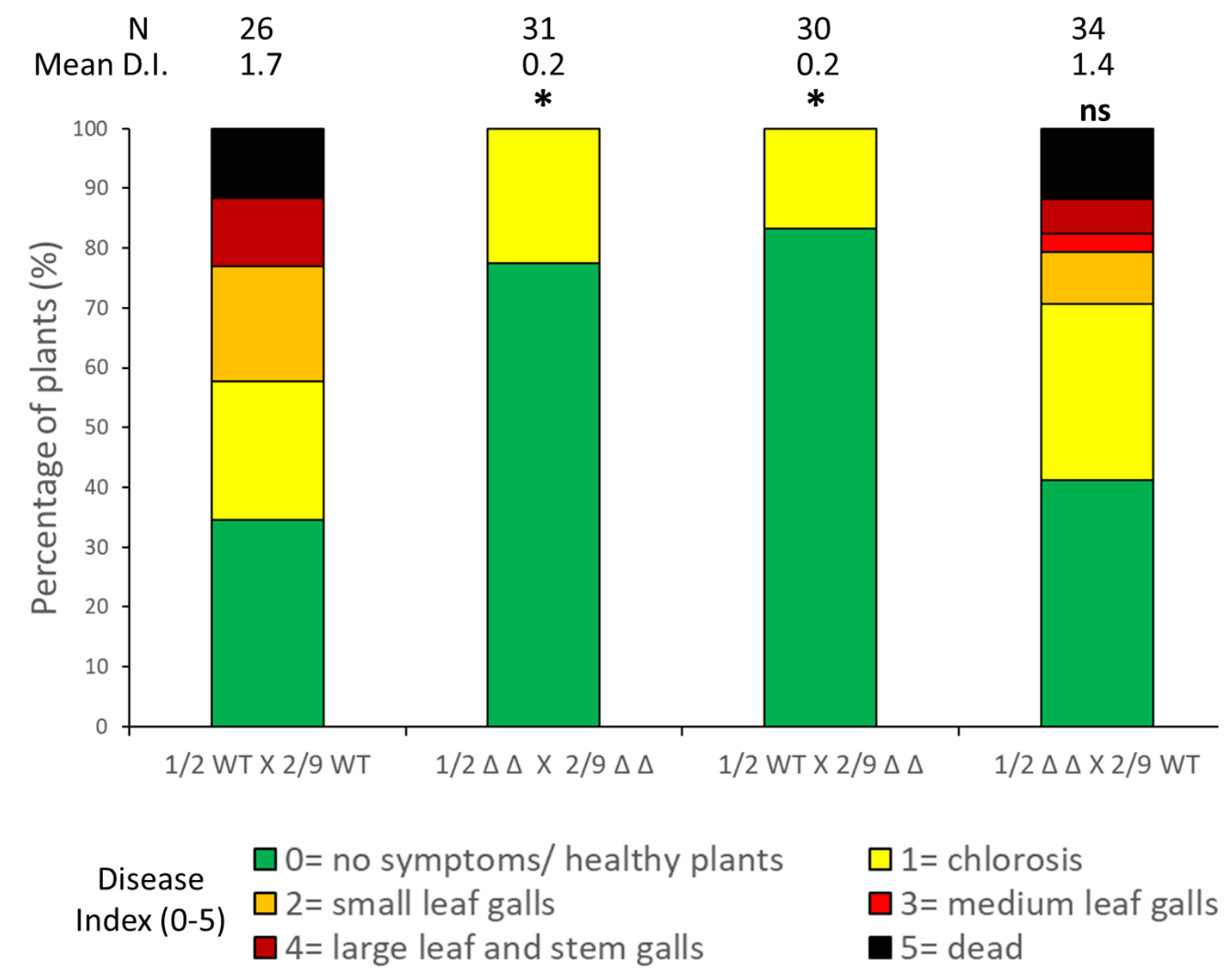

Figure 4. 5 Pathogenicity assay for $\Delta$ um03848 $\Delta$ um03849 mutants.

The indicated strains of $U$. maydis were pre-mixed and injected into 7-day old maize seedlings. Disease symptoms were scored 14 days post infection (dpi). The graph displays the percentage of plants with specific symptoms of infection. The $\mathrm{X}$ axis indicates the paired (1/2 X 2/9) background that was injected into maize plants. The $\Delta u m 03848 \Delta u m 03849$ strains were also combined with the wild type strain of opposite mating-type and were compared with infections due to both wild types (1/2 WT X 2/9 $W T$ ). The disease symptom was categorized from 0 to 5 as depicted at the bottom of the figure. Numbers on the top $(\mathrm{N})$ represent the number of plants infected and mean for disease indices (DI). These data were analyzed using Kruskal-Wallis test followed by post-hoc comparison and an asterisk $(*)$ indicates significant difference $(\mathrm{p}<0.05)$ compared to those where both partners were wildtype strains $(1 / 2 X 2 / 9)$. The significant reduction $(\mathrm{p}<0.05)$ of plant disease symptoms was seen in $1 / 2 \Delta u m 03848 \Delta u m 03849 X$ 2/9 $\Delta u m 03848 \Delta u m 03849$ and 1/2 WT X 2/9 $\Delta u m 03848 \Delta u m 03849$. 
Furthermore, the effect of $\Delta u m 03848 \Delta u m 03849$ on the virulence of the fungus was measured by the two different plant growth parameters: height of the plant and total number of leaves. These parameters in the plants infected with $\Delta u m 03848 \Delta u m 03849$ strains were compared with those infected with the corresponding wild type strains. In figure 4.6 is depicted the difference in the measurements from day of infection to $8 \mathrm{dpi}$ (days post infection). The plants infected with $\Delta u m 03848 \Delta u m 03849$ grew significantly taller (Figure 4.6 A) and had more leaves (Figure 4.6 B), indicating reduced infection, compared to plants infected by wild type strains.

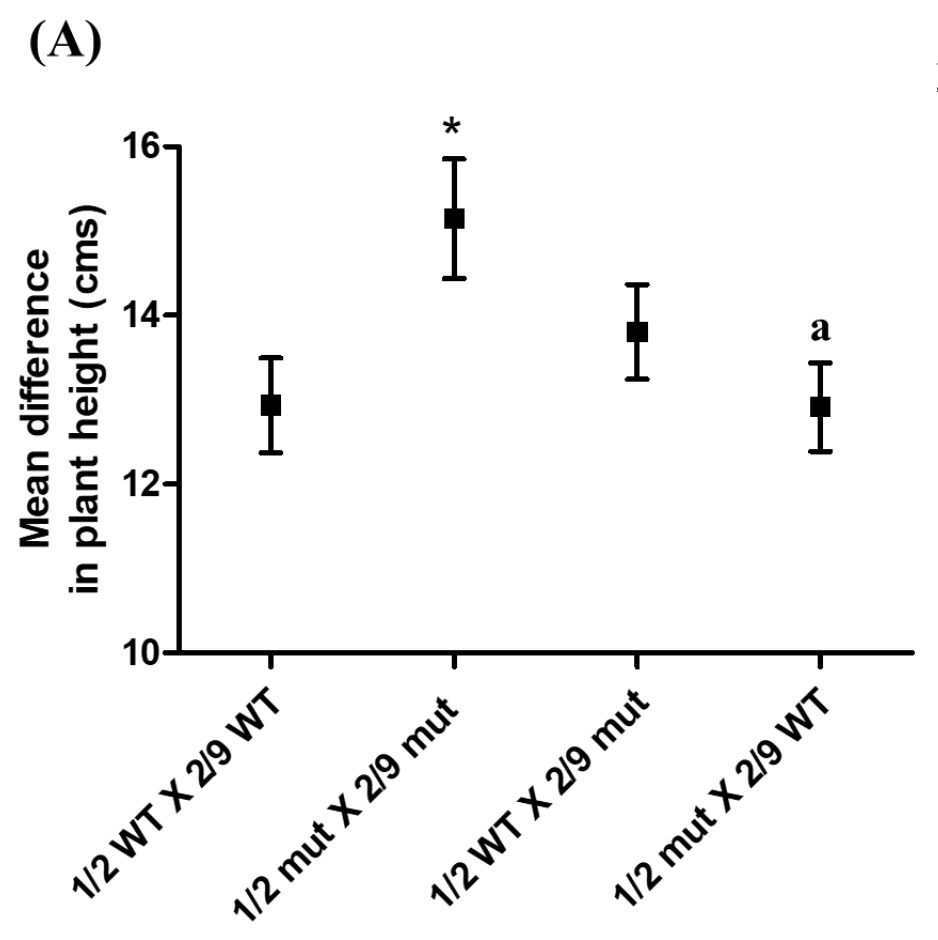




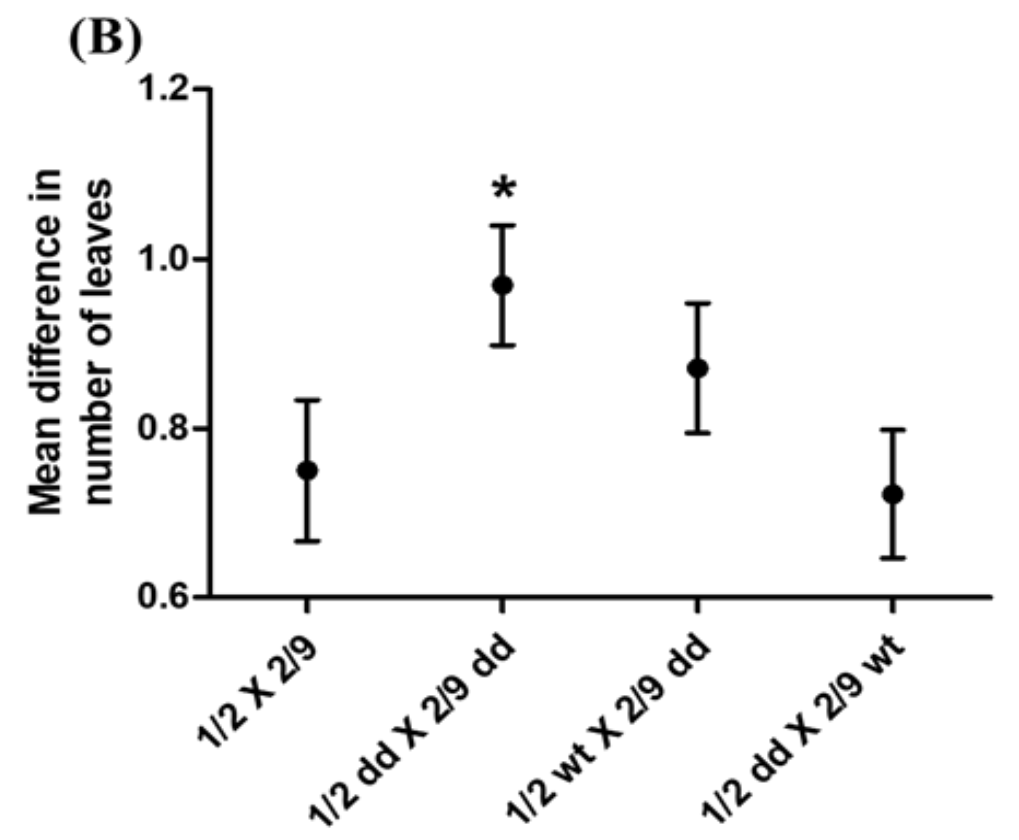

Figure 4. 6 Plant growth measurements for the plants infected by $\Delta u m 03848 \Delta u m 03849$. Effect of $\triangle u m 03848 \Delta u m 03849$ on (A) plant height $(\mathrm{cm})$ and $(\mathbf{B})$ number of leaves were studied. The plants were infected with wild type (1/2 X 2/9), $\Delta u m 03848 \Delta u m 03849$ (1/2 X $2 / 9$ ) and $\Delta u m 03848 \Delta u m 03849$ mating with opposite wild type strains. The mean difference in the height and number of leaves for the plants grown from day of infection to8 dpi is shown in the Y-axis. Statistical analysis was done by ANOVA followed by Tukey's test in GraphPad Prism comparing each group to the wild type (1/2 X 2/9). P value $<0.05$ was considered significant. The asterisk $\left(^{*}\right)$ in the figure shows that the plants infected with $\Delta u m 03848 \Delta u m 03849$ (1/2 X 2/9) have significant increase in plant height and number of leaves when compared to those infected by wild type. "a" represents the significant difference in plant height infected by $1 / 2 \Delta u m 03848 \Delta u m 03849$ mutant with its compatible wild type strain mating partner (1/2 mut X 2/9 WT), compared to plants infected with $\Delta u m 03848 \Delta u m 03849$ (1/2 X 2/9). There were no significant differences in leaf count.

\section{Discussion}

There are several genes involved in the metabolism of nitrate. The nitrate transporter (NRT) transports the extracellular nitrate, and nitrate is then reduced into ammonia by a two-step reduction process, where nitrite reductase (NiR) is involved in the second step of reduction. Nitrate, the first compound in the pathway, induces nitrite 
reductase synthesis while ammonium represses the enzyme at the transcriptional level ${ }^{185}$. We have observed the induction of NRT, um03849, (Chapter III) and NiR, um03848, (Chapter II) genes in $U$. maydis when the fungus was grown in low ammonium medium. In this chapter we investigated the combined roles of genes um03848 and um03849 in growth characteristics, mating and virulence of fungus. To study the phenotypes of haploid $\Delta u m 03848 \Delta u m 03849$ strains, the mutants were grown in low ammonium medium, different sources of nitrogen and different stress media. The phenotypes were compared to the respective wild type progenitor strains grown under the same conditions.

Nitrogen sources determine the morphology of many fungi and especially in dimorphic fungi, nitrogen quality and scarcity are factors that induce filamentation ${ }^{67,86}$. For the filamentation assay, $\Delta u m 03848 \Delta u m 03849$ mutants and wild type strains were grown on low ammonium medium for 4 days. When the individual colonies were observed under a microscope, $\Delta u m 03848 \Delta u m 03849$ mutants showed longer filaments as compared to their respective wild type strains (Figure 4.1). The ability of $U$. maydis to sense and transport nitrogen is critical, and ammonium is a preferred source of nitrogen for many fungi. U. maydis has been reported to produce haploid filaments on nitrogen limited agar media ${ }^{81}$. The higher degree of haploid filamentation on low ammonium medium could represent cells foraging for the nutrients while under starvation. The ump1 and ump2 genes encode the ammonium transporters in U. maydis. Ump2, the highaffinity ammonium transporter, is responsible for the characteristic haploid filamentation phenotype on low ammonium ${ }^{79}$. The overexpression of ump 2 leads to even more filamentous growth of haploid cells on low ammonium ${ }^{65}$. The relationship of other components in the nitrogen assimilation pathway for $U$. maydis have yet to be directly 
connected to Ump2, but the phenotypes of the $\Delta u m 03848 \Delta u m 03849$ strains on low ammonium suggest that the nitrate transporter and/or putative nitrite reductase may at least indirectly be involved in the same pathway leading to haploid filamentous growth.

The ability of haploid $\Delta u m 03848 \Delta u m 03849$ strains to utilize different sources of nitrogen was tested. The serially diluted wild type and deletion mutants were spotted onto minimal media incorporated with $0.3 \%$ of either ammonium, urea, nitrate, or no nitrogen provided. The $\Delta u m 03848 \Delta u m 03849$ strains showed reduced ability to grow in medium with nitrate or urea as a source of nitrogen (Figure 4.2). This indicates that the deletion of NRT and NiR reduces but does not completely inhibit the growth of $U$. maydis in the medium with nitrate as a sole source of nitrogen. In Arabidopsis thaliana, the nitrate reductase is required for the uptake of nitrate ${ }^{186}$. However, such interaction has not been seen in fungal systems. Since $U$. maydis is a biotrophic plant pathogen, there could be an evolutionary adaptation to utilize the host nutrients while inside the plants. Therefore, logical future experiment would be to delete the entire nitrate assimilatory gene cluster NRT-NR-NiR to better understand the utilization of nitrate by $U$. maydis .

In many organisms, the response to stress is related to needs in each ecological niche. The physiological response of most phytopathogenic fungi to various stress conditions is still unknown. We studied the ability of mutant strains to grow in a medium containing $1 \mathrm{M} \mathrm{NaCl}$ or $1 \mathrm{M}$ sorbitol or $1 \mathrm{mM}$ Congo red and compared their abilities to those of their respective wild type strains. Here, the $\mathrm{NaCl}$ and sorbitol provided osmotic stress, while Congo red is a cell wall stress agent. The Congo red acts on fungi by binding to nascent chitin chains, thereby inhibiting the enzymes that connect chitin to $\beta$ 1,3-glucan and $\beta$-1,6-glucan. This results in the weakening of cell wall or affecting the 
cell wall integrity ${ }^{163,187,188}$. We observed that the ability of haploid $\Delta u m 03848 \Delta u m 03849$ cell to grow in medium containing Congo red was reduced (Figure 4.3). However, the inhibition of growth depended on the size of inoculum, with more dilute samples being more strongly inhibited, a finding previously observed in different fungi ${ }^{163,189}$. Usually, sorbitol acts as an osmotic stabilizer in such conditions and maintains the cell wall integrity affected by Congo red ${ }^{163,190}$. However, sorbitol does not dissociate into ions in liquids and is assimilated by yeast. Because of this, it can also be used as an osmotic stress agent ${ }^{110,191}$. Sodium chloride $(\mathrm{NaCl})$ similarly provides osmotic or saline stress and is commonly used in the laboratory to increase medium osmolarity. Salt stress can imbalance the osmotic potential in fungi resulting in water deficit and ionic toxicity to the cell ${ }^{110,111}$. Many organisms, except mammals, accumulate the disaccharide trehalose in response to extreme environment changes ${ }^{192}$. Haploid U. maydis cells grown in sorbitol and $\mathrm{NaCl}$ stress conditions were observed to accumulate trehalose and had higher activity of the trehalase, an enzyme that catalyzes conversion of trehalose into glucose ${ }^{193}$. In our study, we observed that wild type $U$. maydis has high tolerance to the different stress conditions tested. However, the $\Delta u m 03848 \Delta u m 03849$ strains comparatively showed reduced tolerance to the different stressors (Figure 4.3). Hence the interaction of NiR and NRT genes (um03848 and um03849) in U. maydis may induce the protective responses against environmental stresses and longevity of fungal cells while their absence impairs survival under such stress conditions.

Numerous proteins have been classified as osmosensors. Some of these proteins are transmembrane transporters whose function is controlled by mechanical stimulation or changes in medium osmolarity. Moreover, other groups of proteins connect and 
control the signaling pathways for the osmoadaptive responses ${ }^{194}$. When $U$. maydis was grown at high temperature, along with sorbitol and salt stresses, there an accumulation of proteins involved in carbohydrate/amino acid metabolism, protein folding, redox regulation, ion homeostasis and stress response. Other proteins of the 14-3-3 protein family were also accumulated ${ }^{193}$, which are involved in cell signal transduction and apoptosis ${ }^{195}$. Thus, the osmoadaptation, cell wall integrity and dikaryotic filamentation seem to be highly coordinated processes and interlinked with the nitrate assimilation pathway in $U$. maydis. Therefore, integrated study of the nitrate assimilation pathway with the many uncharacterized genes involved in stress response is required.

In the mating efficiency assay, the wild type strains of opposite mating types form a characteristic white "fuzz" in a 1\% PDA charcoal plate indicating successful mating and formation of dikaryotic filaments. When $1 / 2 \Delta u m 03848 \Delta u m 03849$ X $2 / 9$ WT was spotted onto this medium, it also produced white "fuzz". However, 1/2 WT X 2/9 $\Delta u m 03848 \Delta u m 03849$ and $\Delta u m 03848 \Delta u m 03849(1 / 2 \times 2 / 9)$ were not able to form white "fuzz" (Figure 4.4). Therefore, the deletion of both genes involved in nitrate assimilation impairs the mating efficiency of $U$. maydis. Similarly, in the plant pathogenesis assay, $\Delta u m 03848 \Delta u m 03849$ mutants showed reduced virulence to the plant, as measured by Disease Index and percent disease symptoms (Figure 4.5). This was also indicated by the healthier plant growth when infected by $\Delta u m 03848 \Delta u m 03849$ mutants as measured by plant height and number of leaves (Figure 4.6). However, when 1/2 $\Delta$ um03848 sum03849 was mixed with 2/9 wild type and injected into plants, the infection was similar to that of wild type strains (1/2 X 2/9). Interestingly, this finding correlates with the significantly higher filamentation by $1 / 2 \Delta u m 03848 \Delta u m 03849$ on low ammonium 
(Figure 4.1). Thus, the filamentous growth under ammonium limited conditions in the laboratory could be of functional importance in the pathogenicity of the fungus. In $S$. cerevisiae, Mep2, ammonium permease is involved in pseudohyphal, filamentous and invasive growth of yeast ${ }^{196}$. Mep2 overexpression enhances pseudohyphal growth and induces transcription of genes of the MAPK pathway, even under non-inducing condition ${ }^{197}$. Similarly, the overexpression of ump2 in $U$. maydis increases filamentation in low ammonium medium and induces filaments even in high ammonium ${ }^{82}$. The expression of genes involved in mating and pathogenicity pathways correlated to the ump 2 expression level ${ }^{65}$. Therefore, in a pathogenic fungus, such as $U$. maydis, there is a direct relation in sensing nutrient availability, fungal development, cellular differentiation and pathogenicity.

The nitrogen limitation during in planta growth of the phytopathogen induces the proliferation and sporulation of the fungus ${ }^{55,87}$. The Nitrogen Metabolite Repression (NMR) program serves as a master controller of the transcriptional regulation of permeases and catabolic enzymes needed for utilization of secondary nitrogen sources. During in planta growth of fungus, the nitrogen starvation and NMR regulation is a stage-specific or recurring condition where the transition periods such as appressorium formation, proliferation, or sporulation are likely to increase the demand for energy ${ }^{134}$. Nit2, one of the nitrate assimilation-specific positive regulators of NMR in U. maydis is involved in the formation of dikaryotic filaments of the fungus in planta ${ }^{6}$. The dikaryotic filaments are pathogenic and must form appressoria, a structure to penetrate host tissue. Therefore, deletion of nit2 gene delays the dikaryotic filamentation and, in turn, severely impairs the pathogenicity of $U$. maydis $^{6}$. Hence, studying the different phases of 
development of the $\Delta u m 03848 \Delta u m 03849$ mutants is important to know when nitrate utilization is involved during in planta stages of the fungus and how this affects the pathogenicity.

The plant infection pattern observed in the double mutant, i.e., reduced virulence based on the 2/9 background (Figure 4.5), is similar to that seen in $\Delta u m 03849$ mutants (Figure 3.6), while this is opposite to that shown by $\Delta u m 03848$ strains (Figure 2.9.). In the assimilatory reduction of nitrate to ammonia, nitrate transporter (NRT) is the first step, where nitrate is taken up by the cell. Then, nitrate reductase (NR) and nitrite reductase $(\mathrm{NiR})$ are involved in the reduction of cellular nitrate. Since, um03849, NRT is the initial gene in response to nitrate assimilation, the infection by um03849 or double deletion ( $\triangle u m 03848 \Delta u m 03849$ ) should be independent of the phenotype shown by $\Delta u m 03848$, as the latter gene is downstream in this assimilation pathway and likely would have little additional effect if nitrate is not transported into the cells.

A phylogenetic study was performed to test the evolution and conservation of stress signaling molecules among different fungal species from highly divergent niches. It was found that the general and central components of the osmotic, oxidative, and cell wall stress signaling pathways are relatively well conserved even though the upstream sensors and downstream transcriptional regulators have diverged significantly ${ }^{111}$. In $S$. cerevisiae, the MAPK pathway is required for the responses to osmotic stress and cell wall integrity ${ }^{198}$. The core components of stress pathways such as a Rho1 (G-protein), Pkc1 (Protein kinase) and Slt2 (MAP kinase) are highly conserved among different fungi 111. MAPK and cAMP-PKA pathways regulate the mating and virulence pathways in many phytopathogenic fungi including $U$. maydis and are often associated with nitrogen 
starvation. In the absence of the mating partners in U. maydis, the same MAPK pathway leads to haploid filamentation ${ }^{68,199}$. Rho1 GTPase interacts with the ammonium transporter, Ump2, and controls the filamentation and pathogenesis in U. maydis ${ }^{200}$. Despite plentiful information on the MAPK cascade, there are still many unsolved questions about this signaling pathway. The mechanisms by which signaling through MAPK pathways are integrated with other types of pathways are still ongoing studies. The $\Delta u m 03848 \Delta u m 03849$ mutants showed higher haploid filamentation, reduced growth in stress medium, inability to mate between compatible partners and inability to induce tumors in the host plant. This indicates the complex interactions among the different components of the signaling pathway, which need to be explored. 


\section{CHAPTER V \\ DISCUSSION}

Nitrogen is an essential component for most of the biological macromolecules in organisms. In addition, nitrogen is important for aspects of growth, development, reproduction 201,202 , the production of secondary metabolites and the regulation of virulence determinants in many phytopathogenic fungi ${ }^{87,201,203,204}$. Therefore, the availability of accessible forms of nitrogen or being able to assimilate complex nitrogen sources into the essential macromolecules is important for all living organisms. Consequently, the regulatory response to the nitrogen availability is complex and probably involves various parallel signaling and regulatory systems.

Nitrogen assimilation is the formation of organic nitrogen compounds like amino acids from inorganic nitrogen compounds present in the environment. Organisms like plants, fungi and certain bacteria that are not able to fix nitrogen gas depend on the ability to assimilate nitrogen ${ }^{1}$. Nitrate is one of the major forms of assimilable nitrogen in the biosphere. Nitrate assimilation starts with the uptake of nitrate and then reduction into ammonium catalyzed by the proteins nitrate transporter (NRT), nitrate reductase (NR) and nitrite reductase (NiR). Therefore, nitrate utilization requires significant energy resources for transport into the cell and conversion of the nitrogen from +5 to -3

oxidation state ${ }^{143}$. Thus, the metabolism of nitrate is a highly regulated mechanism and 
catabolic enzymes are selectively induced in the absence of preferred nitrogen sources and presence of nitrate.

Nitrate is abundant in croplands and often supplied in fertilizers. Thus, nitrate is one of the major nitrogen sources for the crop plants such as maize/corn ${ }^{205}$. The phytopathogen Ustilago maydis establishes a biotrophic relationship with its host corn plant to complete its lifecycle. During this process, the fungus gains access to the resources in plant by invading the host tissues ${ }^{83}$ which they use to proliferate and induce tumors. The biotrophic fungi accomplish this by manipulating host plants to shift nutrients to the sites of infection and themselves by expressing the required transporters for the nutrients ${ }^{206}$. In the fungal plant pathogens such as Stagonospora (syn. Septoria) nodorum (Berk.), causative fungi of wheat blotch and Phytophthora infestans, hemibiotrophs for potato and tomato, the NRT and nitrate assimilating genes were highly upregulated during early- and mid-infection in the host plant ${ }^{206,207}$. Similarly, in the maize plant infected by $U$. maydis, the high amount of nitrogen is rerouted from the systemic leaves into tumors to overcome the nitrogen requirement for fungal development ${ }^{95}$. In addition, the crop infection by $U$. maydis has significant effect on the economy. The economic consequence is more on sweet corn than in field corn. If there is a single smut gall in a sweet corn, it is usually not marketable because of "cosmetic injury". Some consumers don't accept the corn if there is presence of black teliospores on husk leaves even though ears are not damaged. So, there are additional costs incurred to remove teliospores from husk leaves. Similarly, when sweet corn is grown for processing like canning and freezing, the fields are not harvested if there is even a moderate amounts of ear galls ${ }^{208}$. Hence, even a low incidence of smut can result in substantial losses. 
Therefore, understanding the fungal ability to utilize and metabolize nitrate as the source of nitrogen is important to understand its mechanism to infect and proliferate inside the host plants.

The genome sequence analysis performed on the haploid strain of basidiomycete Ustilago maydis-521 was published in $2006^{46}$. The availability of genome sequence of U. maydis as well as various genetic and functional tools offered an excellent opportunity to unravel the molecular secrets of this fungus including the study of nitrogen metabolism. There are studies showing the important roles of ammonium transporters in the mating and pathogenicity of the $U$. maydis ${ }^{65,82}$. The deletion of high affinity ammonium transceptor, ump2, severely reduces the fungal ability to form haploid filament, in absence of mating partner. In presence of compatible partner, the deletion of ump2, affects the ability to form dikaryotic filament (mate) and virulence on the host plant ${ }^{82}$. However, the mechanism of uptake and utilization of nitrate in $U$. maydis have not yet been explored. The current study sought to provide better understanding of the assimilation of nitrogen in $U$. maydis via nitrate metabolism pathway.

The advancements on the genome and transcript level analysis aided identification of the potential genes encoding nitrate permease/transporter(UMAG_11105 or um03849) and nitrite reductase (UMAG_11104 or um03848) in $U$. maydis ${ }^{47,55,209}$. A gene encoding nitrate reductase (narl, um03847) has been previously identified and studied ${ }^{184}$. The genes coding for nitrate reductase, nitrite reductase and a nitrate transporter are located in one gene cluster in $U$. maydis, suggesting the coordinated regulation for utilization of nitrate ${ }^{55}$. The studies by Ho et al., 2007 and Horst et al., 2012 have shown the significant upregulation of UMAG_11105, NRT, and UMAG_11104, NiR, genes in U. maydis when 
grown under nitrate minimal medium or no nitrogen medium. Therefore, the current study focused on the characterization of $U$. maydis NRT and NiR based on various phenotypes observed. To elucidate the functions of NRT and NiR as an assimilatory gene, deletion mutants of NRT and NiR were constructed in $U$. maydis strains of opposite mating type so as to allow characterization of the phenotypes in both genetic backgrounds, but also to allow examination of effects on mating, if any. The double deletion of NRT and $\mathrm{NiR}$ genes were also constructed in compatible mating partners. The $U$. maydis deleted mutants were then evaluated in terms of different growth, morphological phenotypes, and eventually focusing mostly on mating and virulence.

The deletion of gene um03848 (NiR) or um03849 (NRT) or the deletion of both genes reduced the ability of $U$. maydis to grow on the media with nitrate as a sole source of nitrogen (Figures 2.5, 3.3 and 4.2). However, they were still able to grow on those media. This indicated that $U$. maydis can sense and take up nitrate/nitrite with an independent, as yet unknown, transport system. This coincides with the concept that multiple nitrate transporter systems exist in an organism, which has also been confirmed in different plants, algae and yeast 1,144,147,185,210,211. Identification and characterization of such transporters or the nitrogen metabolic regulator in U. maydis remain to be explored. Furthermore, the regulation of genes involved in nitrate assimilation in Fusarium fujikuroi indicates the fungus is able to directly sense nitrate in an NRT-independent, but NR-dependent manner ${ }^{100}$. This conclusion is based on nitrate-induced nuclear translocation of a NMR (Nitrogen Metabolic Repression) component in $\triangle$ NRT mutants 100 . 
In addition, the reduced growth of deleted mutants on nitrate medium was comparable to the growth of wild type strains grown on no nitrogen medium. So, the minimal growth of deleted mutants could also have been because of the fungal ability to grow in a medium without nitrogen source ${ }^{79,99}$, and irrespective to unknown nitrate metabolism pathway. Similarly, the growth of the double gene deleted mutant $(\Delta u m 03848 \Delta u m 03849)$ in medium with urea as a source of nitrogen (Figure 4.2) was impaired compared with that shown by single mutants (Figure 2.5, 3.3). This indicates that the deletion of nitrate metabolization genes might affect the transport or utilization of other nitrogen sources.

Horst et al., 2010 mentioned, from the unpublished data of their team, that the nitrate reductase mutants in the SG200 background were fully pathogenic and $U$. maydis in planta may not use nitrate as a source of nitrogen. However, in our study, we found that the deletion of either nitrate transporter (um03849) or nitrite reductase (um03848) significantly reduces the pathogenicity to the plant. However, when the mutants were mixed with the compatible wild type strains, the reduced virulence was observed in a background specific manner (Figures 2.10 and 3.6). The effect on virulence of deletion of um03848 or um03849 or both genes was also confirmed through alternate methods such as measuring plant growth. Based on the measurements on plant height and number of leaves developed, the $\Delta u m 03848$ or $\Delta u m 03849$ infected plants were healthier compared to the wild type infected plants. This indicates that the ability of $U$. maydis to transport or utilize nitrate affects virulence against the host plant. Our result is similar to the study performed on Phytophthora infestans where silencing the expression of the nitrate 
assimilatory gene cluster (NRT, NR, NiR) reduced its virulence to the host plant with no symptoms or a few necrotic lesions ${ }^{206}$.

Studies on the growth of corn plants have shown that the nitrates may accumulate in corn during stress conditions such as drought, high temperature, low humidity, large amounts of nitrogen in the fertilizer ${ }^{136,212,213}$. When such plants are infected with the fungus that has reduced expression or deletion of nitrate transporter or nitrate metabolizing genes, the fungus wasn't able to colonize or cause infection to the plants ${ }^{137}$. This could be because of two reasons: the pathogen is not able to use nitrate for nutrition and/or toxicity of high levels of nitrate. Hence, the nitrate profiling should be done in the plants on the site of infection. Also, it will be good to investigate the effects on virulence when plants are grown on nitrogen-poor soil. These experiments are important to understand whether the reduced pathogenicity, observed for the fungus with nitrate assimilatory genes deleted, was solely due to inability of fungus to utilize nitrate but not because of toxic effect of nitrate accumulation in the plant.

In the pathogenesis assay, we observed that the nitrate assimilatory genes affect the virulence in plant. The reduction of virulence to the plant was specific to a particular genetic background of the fungus. In $\Delta u m 03848$, the deletion in $1 / 2$ reduced the virulence when paired with $2 / 9$ wild type strain. However, in $\Delta u m 03849$ or $\Delta u m 03848 \Delta u m 03849$, deletion in 2/9 significantly affected the pathogenicity when this mutant was mixed with $1 / 2$ wild type strain and used for infection. We observed that the $\Delta u m 03848 \Delta u m 03849$ background specific reduction of virulence was similar to that of $\Delta u m 03849$; however, this was opposite to what was observed with just $\Delta u m 03848$. Although the different genetic backgrounds used for these studies differed in their respective mating-type loci, at 
present we do not have any direct indication that these differences per se are the cause of the differential outcomes for deletion mutants for $\Delta u m 03848, \Delta u m 03849$, or the double deletion strains. The nitrate transporter, um03849, is involved in the first step of the nitrate utilization process. So, it can be expected that the deletion of um03849 would disable the cellular transport of nitrate and in turn should repress the genes involved in nitrate assimilation. To test this hypothesis, we performed qRT-PCR on $\triangle u m 03849$ for the expression of um03848, nitrite reductase. We observed that deletion of NRT, upregulates the expression of $\mathrm{NiR}$ when grown on low ammonium medium (Figure 5.1). This indicates that nitrite reductase could have an independent sensor or regulatory role under nitrogen starvation conditions, rather than just as a nitrate transporter. In the filamentous fungus Aspergillus nidulans nitrite is transported via nitrate transporter as well as high-affinity nitrite-specific transporter ${ }^{214}$. The tomato pathogen Fusarium oxysporum f. sp. lycopersici also possesses separate nitrate and nitrite transporters ${ }^{215}$.
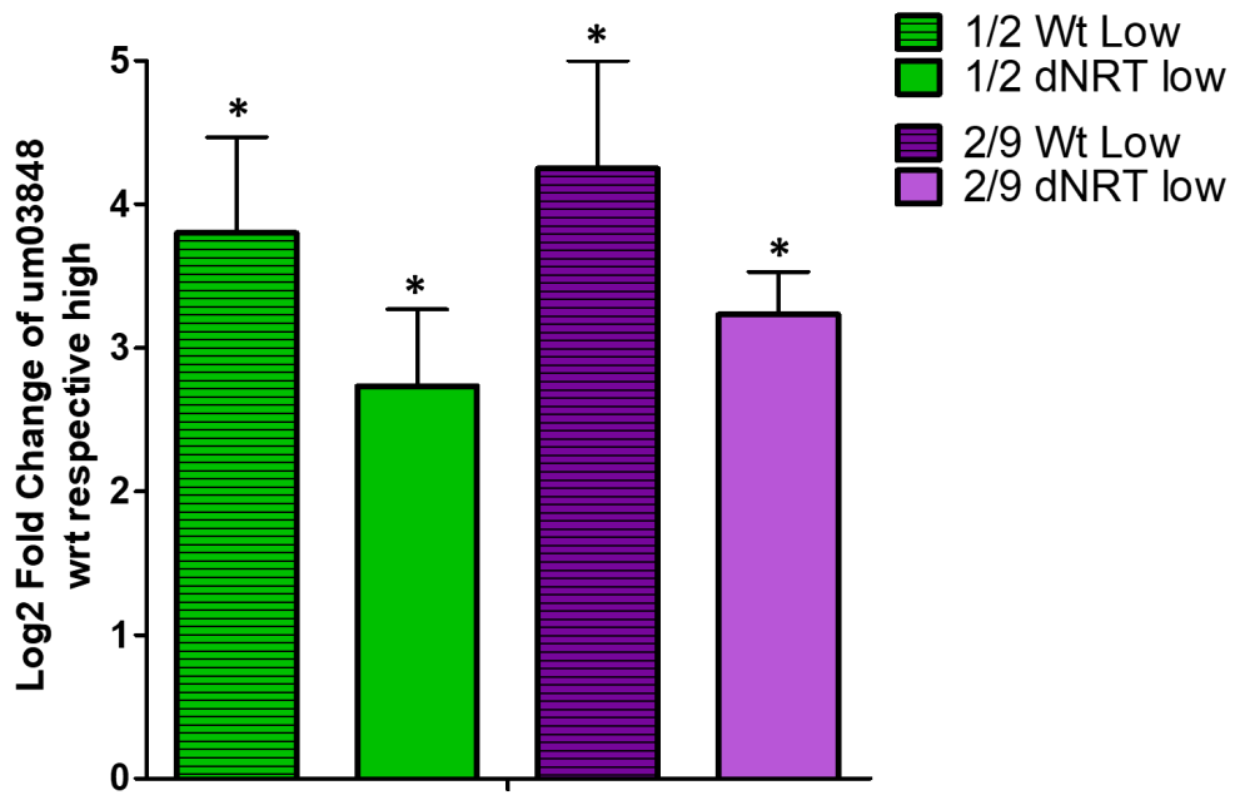

Figure 5. 1 The average log2 fold changes in um03848 gene expression by $\Delta u m 03849$ mutants grown under low ammonium condition. 
The wild type and $\triangle u m 03849$ strains on 1/2 (green bar) and 2/9 (purple bar) backgrounds were grown under low ammonium and high ammonium conditions. The qRT-PCR was performed for $u m 03848$, nitrite reductase gene, on the total RNA extracted from these strains. The $\log 2$ fold changes in expression of the strains grown under low ammonium were normalized against eif 2 and are expressed with respect to (wrt) the same strain grown under high ammonium. Bars represent the averages of biological triplicates and standard errors are indicated in the graphs. One-way ANOVA followed by Tukey's Multiple Comparison Test was performed in GraphPad Prism. P-value $<0.05$ is considered as significant. $*$ on top of bars indicates the significant difference in the expression level of target gene for the strain grown on low ammonium with respect to same strain grown on high ammonium. However, there was no significant difference in the expression level of the mutant on low when compared with wild type on low.

The $\Delta u m 03849$ or $\Delta u m 03848 \Delta u m 03849$ mutants showing similar phenotype in terms of virulence to the plant could be also related to the structural arrangements and the transcriptional regulation of the nitrate assimilatory genes. Among the filamentous fungi, there are differences in the organization of the genes involved in nitrate assimilation pathway $5,201,204,216,217$. The structural genes of the nitrate assimilation pathway in $A$. nidulans are clustered in the order crnA-niiA-niaD, where $\operatorname{crnA}$ is involved in nitrate uptake and niiA and niaD encode nitrite and nitrate reductase, respectively. However, niiA and niaD are divergently transcribed from an intergenic region, while $\mathrm{crnA}$ and niiA are adjacent and transcribed in the same direction ${ }^{145,216}$. For $N$. crassa, in contrast, the genes for nitrate (nit-3) and nitrite (nit-6) reductases are in separate chromosomes and unlinked and no uptake gene has been reported ${ }^{218}$. In U. maydis, the nitrate assimilatory genes are known to be closely associated ${ }^{56}$, although the directions of transcription have not been determined. Clustering of the functionally related genes may be selectively favorable as it facilitates the coordinated expression of the genes and makes their separation less likely during recombination events. 
We analyzed the in-silico protein interactions between the nitrate assimilatory pathway with other nitrogen metabolism pathways in $U$. maydis. The protein-protein interactions were predicted for the $U$. maydis proteins that are known or putative transporter/permease in nitrogen metabolism pathway. The STRING database (www.string-db.org) predicted putative interactions between high affinity ammonium transporter (ump2, um05889) and the nitrate assimilatory genes: NRT (um03849), NR (um03847) and NiR (um03848) (Figure 5.2). This provides putative mechanistic support for the significant downregulation of $u m p 2$ in $\Delta u m 03848$ mutant strains (Figure $2.11 \mathrm{~A}$ ). However, protein interaction showed co-expression of um03847 encoding nitrate reductase (Nar1) and um05889 encoding Ump2. For these reasons, it will be interesting to analyze all three nitrate assimilatory genes along with the ammonium transporter Ump2 to understand the interaction between these genes in nitrogen limited conditions.

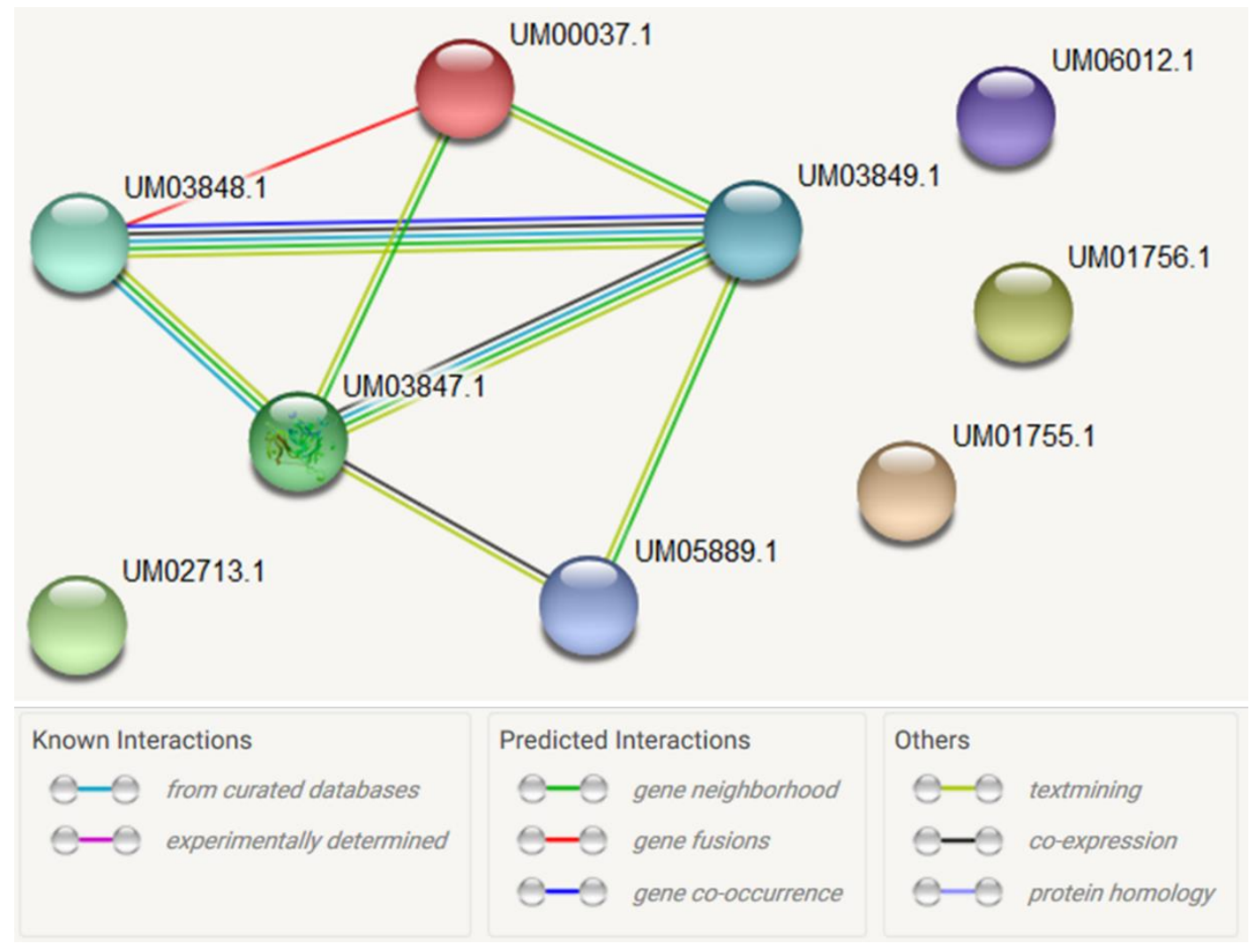




\begin{tabular}{|c|c|}
\hline UM00037.1 & Uncharacterized protein; NirD domain (554 aa) \\
\hline UM01755.1 & Urea permease; DUR3 (674 aa) \\
\hline UM01756.1 & Putative Purine Transporter AzgA (614 aa) \\
\hline UM02713.1 & Pheromone response factor Prf1 (840 aa) \\
\hline UM03847.1 & Nitrate reductase [NADPH] (983 aa) \\
\hline UM03848.1 & Putative nitrite reductase (602 aa) \\
\hline UM03849.1 & Nitrate transporter (607 aa) \\
\hline UM05889.1 & High affinity ammonium transporter (487 aa) \\
\hline M06012.1 & Probable general amino acid permease (548 aa) \\
\hline
\end{tabular}

Figure 5. 2 Prediction of protein-protein interaction between nitrogen transporter/ permease in $U$. maydis.

The STRING database was used to analyze the potential protein-protein interaction in different nitrogen transporters in $U$. maydis. The circle indicates a protein and lines represent the interaction as depicted in the legend. The table shows the list of proteins that are studied. The Prf1 (um02713), master regulator of mating and virulence, was also analyzed. As expected, it did not appear to have any direct protein-protein interactions with the other analyzed proteins involved in nitrogen assimilation. Because the gene um00037 contains a large domain of NirD and we had previously studied this gene (included in Appendix III), it is analyzed here as well.

To evaluate the reduced pathogenicity of $U$. maydis by the NiR and NRT deleted mutants, the expression of mating targets was assessed on the mutants grown on low ammonium conditions. There was a differential expression of mating targets between $1 / 2$ and 2/9 backgrounds in the um03848 and um03849 mutants (Figures 2.12 And 3.8). However, the deletion of either um03848 or um03849 downregulated the $b$ locus genes and transcription factors Prf1 and Rbf1. Therefore, from the gene expression analysis we can say that $u m 03848$ and $u m 03849$ both interact in some way with $\mathrm{bE} / \mathrm{bW}$ genes which in turn regulates the expression of transcription factors Prf1 and Rbf1 to control the pathogenic development of $U$. maydis. The yeast-to-hypha dimorphic transition in $U$. maydis is regulated by the crosstalk between MAPK and PKA signal pathways. The 
signal from both pathways phosphorylates transcription factor Prf1 which acts initially as the master regulator of the pathogenic process by regulating the expression of $a$ and $b$ loci genes ${ }^{47,68}$. The mating compatibility of $U$. maydis is determined by the gene products of $a$ and $b$ loci where genes from $a$ locus regulates pre-mating and genes from $b$ locus regulate post-mating ${ }^{61}$. The homeodomain proteins $\mathrm{bE}$ and $\mathrm{bW}$ encoded from $b$ genes form a heterodimer $\mathrm{bE} / \mathrm{bW}$ after fusion with compatible partner ${ }^{59}$ which further induces the transcriptional regulator Rbf $1{ }^{138}$. The $b E$ and $b W$ genes are generally upregulated in $U$. maydis grown on low ammonium conditions. However, in the mutant backgrounds that showed reduced pathogenicity, we have observed downregulation of either or both $b E$ and $b W$ genes. Similarly, $r b f l$ is significantly upregulated in wild type cells grown on low ammonium medium whereas it is downregulated in um03848 and um03849 deleted mutants (Figures 2.12 and 3.8). The expression level of the gene encoding the high affinity ammonium transceptor in $U$. maydis, ump2, has an important role in transmitting information to the developmental stages of fungus. Also, ump2 and the $b$ locus genes are tied together at the transcriptional level to induce the transcription factors Prf1 and Rbf $1{ }^{65}$. Furthermore, overexpression of ump 2 in a mutant with the entire $b$ locus deleted rescued the loss of filamentation phenotype ${ }^{82}$. In $\triangle u m 03848$ and $\Delta u m 03849$ mutants grown on low ammonium, the ump2 was upregulated compared to the mutants grown on high ammonium while it was downregulated compared to wild type grown under the same conditions (Figures 2.12 and 3.8).

From this study, we observed that the gene encoding nitrate transporter, um03849 and nitrite reductase, um03848 play different roles in transcriptional regulation of $U$. maydis for mating and virulence. Nevertheless, to understand the background-based 
reduction of virulence, the expression of the mating/ virulence genes should be analyzed 12 to $24 \mathrm{~h}$ post infection in the plant because this is the determining phase for the fungus to establish biotrophic relation to host. The ability of the mutants to form appressorium, the specific structure to penetrate host plant ${ }^{182}$, should be characterized. Also, knowing that tumors can be induced by few fungal cells ${ }^{76}$, the lack of tumor formation in $\Delta u m 03848$ mutants can be characterized further by quantification of fungal biomass inside the host plant. Further analysis of tumor development and viability of spores can be studied in the virulence levels caused by specific background of mutants. Also, little is known on how $U$. maydis assimilates or uses nitrogen after entering the host. Besides, nitrate is not the preferred source of nitrogen for U. maydis. The transcriptome analysis for the $U$. maydis inside the 2 dpi host plant showed the induction of five transporters: two putative urea permeases, two putative oligopeptide transporters and candidate methylammonium permease and ump2 whereas nitrate transporter was not induced in such condition ${ }^{219}$. This indicating that nitrate transporter might be induced after 2 dpi infection. This might be reason why $\Delta u m 03849$ mutant where able to induce small tumors in plant although overall reduced the virulence to plant compared to wild type (Figure 3.6).

Additionally, the fungus has nitrogen catabolite repression (NCR) as a regulatory strategy to restrict the utilization of unfavored nitrogen sources when a favored nitrogen source is available ${ }^{5}$. AreA in Aspergillus nidulans and Nit2 in Neurospora crassa are well-known regulatory genes of $\mathrm{NCR}^{3,21}$. The Nit2/AreA ortholog has also been identified in U. maydis and it has been shown that deletion of Nit2 affects the ability of the fungus to utilize complex nitrogen sources, including nitrate. The Nit2 deletion also 
reduced the virulence to the plant ${ }^{6}$. Therefore, this indicates that $U$. maydis utilizes the unfavored form of nitrogen inside host cells to be able to proliferate and induce tumors. Thus, the study of the interaction of genes involved in NCR as well as nitrate metabolism will help to understand the underlying regulatory mechanisms for nitrate utilization.

Overall, this study contributes additional information about how the phytopathogen, U. maydis, adapts to growth on nitrogen starvation or no nitrogen conditions. This is the first study evaluating nitrate assimilatory genes in $U$. maydis. We reported that the two genes, um03848 and um03849, serve as nitrite reductase and nitrate transporter, respectively, and that they are involved in nitrate acquisition in $U$. maydis and are induced upon nitrogen starvation. We concluded that $U$. maydis can assimilate inorganic form of nitrogen such as nitrate. Finally, the ability of this fungus to utilize and metabolize nitrate as a source of nitrogen plays an important role in the pathogenicity of the fungus to its host plant. We found that um03848 and um03849 together are also involved in cell wall integrity, osmoregulation and filamentation (Figure 5.3). As nitrate is the readily available assimilable form of inorganic nitrogen, this study opens possible research avenues in the use of the phytopathogen $U$. maydis as a model organism to study the nitrogen assimilation pathway. 


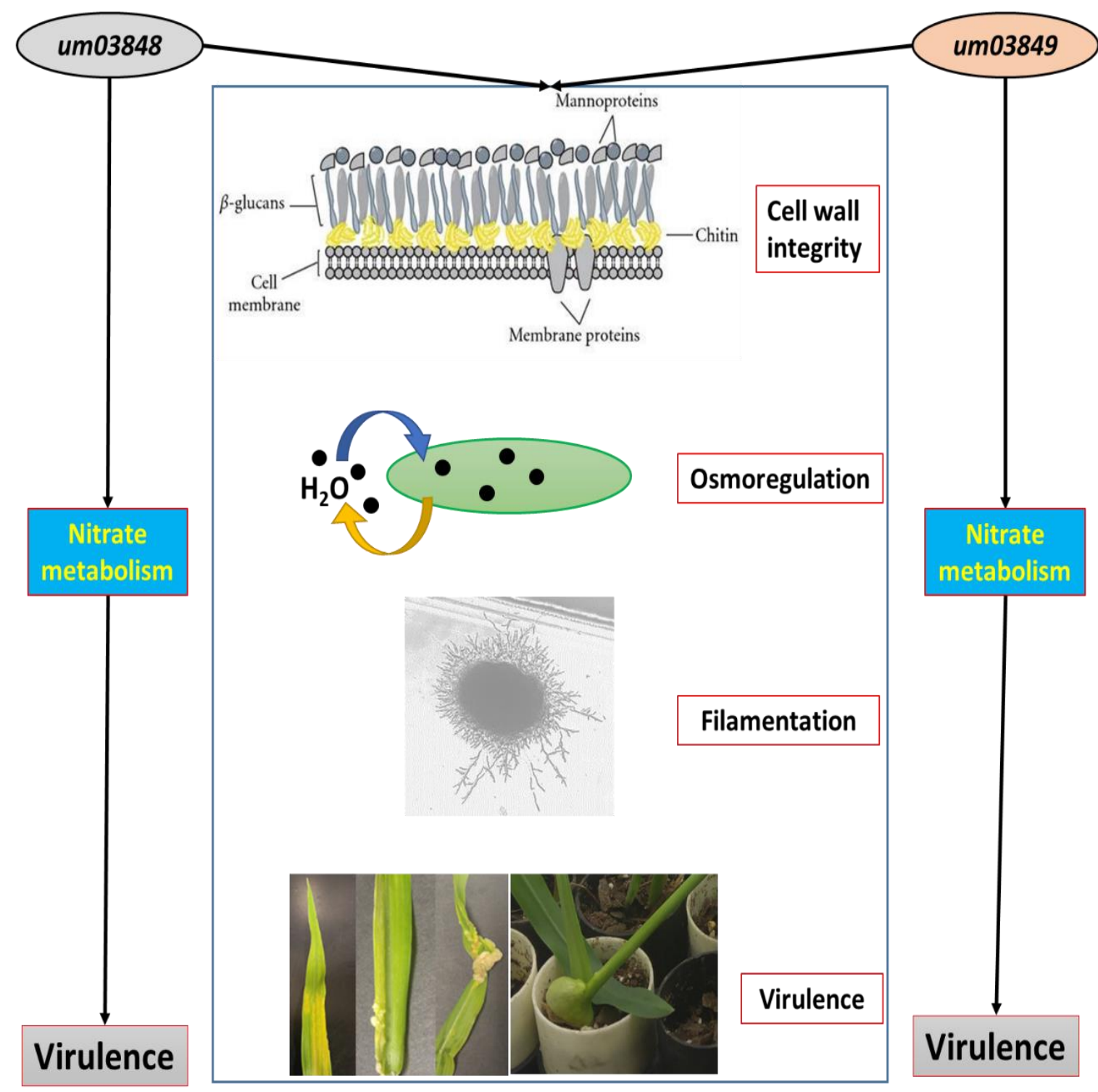

Figure 5. 3 Proposed mode of actions for um03848 and um03849 in U. maydis. 


\section{REFERENCES}

1. Lin, J. T. \& Stewart, V. Nitrate assimilation by bacteria. Advances in Microbial Physiology (1997).

2. Kuypers, M. M. M., Marchant, H. K. \& Kartal, B. The microbial nitrogen-cycling network. Nature Reviews Microbiology (2018). doi:10.1038/nrmicro.2018.9

3. Marzluf, G. A. Genetic regulation of nitrogen metabolism in the fungi. Microbiol. Mol. Biol. Rev. (1997).

4. Koon, H. W., Hynes, M. J. \& Davis, M. A. Recent advances in nitrogen regulation: A comparison between Saccharomyces cerevisiae and filamentous fungi. Eukaryotic Cell (2008). doi:10.1128/EC.00076-08

5. Marzluf, G. Regulation of Sulfur and Nitrogen Metabolism in Filamentous Fungi. Annu. Rev. Microbiol. (1993). doi:10.1146/annurev.micro.47.1.31

6. Horst, R. J. et al. The Ustilago maydis Nit2 Homolog Regulates Nitrogen Utilization and Is Required for Efficient Induction of Filamentous Growth. Eukaryot. Cell 11, 368-380 (2012).

7. Wiame, J. M., Grenson, M. \& Ars, H. N. Nitrogen Catabolite Repression in Yeasts and Filamentous Fungi. Adv. Microb. Physiol. (1985). doi:10.1016/S00652911(08)60394-X

8. Scazzocchio, C. The fungal GATA factors. Current Opinion in Microbiology (2000). doi:10.1016/S1369-5274(00)00063-1

9. Magasanik, B. \& Kaiser, C. A. Nitrogen regulation in Saccharomyces cerevisiae. Gene 290, 1-18 (2002).

10. Cooper, T. G. Transmitting the signal of excess nitrogen in Saccharomyces cerevisiae from the Tor proteins to the GATA factors: Connecting the dots. FEMS Microbiology Reviews (2002). doi:10.1016/S0168-6445(02)00071-2

11. Rai, R. et al. Nuclear Gln3 import is regulated by nitrogen catabolite repression whereas export is specifically regulated by glutamine. Genetics 201, 989-1016 (2015).

12. Georis, I., Tate, J. J., Cooper, T. G. \& Dubois, E. Tor pathway control of the nitrogen-responsive DAL5 gene bifurcates at the level of Gln3 and Gat1 regulation 
in Saccharomyces cerevisiae. J. Biol. Chem. (2008). doi:10.1074/jbc.M708811200

13. Crespo, J. L. \& Hall, M. N. Elucidating TOR Signaling and Rapamycin Action: Lessons from Saccharomyces cerevisiae. Microbiol. Mol. Biol. Rev. 66, 579-591 (2002).

14. Beck, T. \& Hall, M. N. The TOR signalling pathway controls nuclear localization of nutrient- regulated transcription factors. Nature 402, 689-692 (1999).

15. Loewith, R. et al. Two TOR complexes, only one of which is rapamycin sensitive, have distinct roles in cell growth control. Mol. Cell (2002). doi:10.1016/S10972765(02)00636-6

16. Kuruvilla, F. G., Shamji, A. F. \& Schreiber, S. L. Carbon- and nitrogen-quality signaling to translation are mediated by distinct GATA-type transcription factors. Proc. Natl. Acad. Sci. U. S. A. (2001). doi:10.1073/pnas.121186898

17. Chen, J., Zheng, X. F., Brown, E. J. \& Schreiber, S. L. Identification of an 11-kDa FKBP12-rapamycin-binding domain within the 289-kDa FKBP12-rapamycinassociated protein and characterization of a critical serine residue. Proc. Natl. Acad. Sci. U. S. A. (1995). doi:10.1073/pnas.92.11.4947

18. Stan, R. et al. Interaction between FKBP12-rapamycin and TOR involves a conserved serine residue. J. Biol. Chem. (1994).

19. Coffman, J. A., Rai, R. \& Cooper, T. G. Genetic evidence for Gln3p-independent, nitrogen catabolite repression- sensitive gene expression in Saccharomyces cerevisiae. J. Bacteriol. 177, 6910-6918 (1995).

20. Georis, I., Feller, A., Vierendeels, F. \& Dubois, E. The Yeast GATA Factor Gat1 Occupies a Central Position in Nitrogen Catabolite Repression-Sensitive Gene Activation. Mol. Cell. Biol. (2009). doi:10.1128/mcb.00399-09

21. Platt, A. et al. Nitrogen metabolite signalling involves the C-terminus and the GATA domain of the Aspergillus transcription factor AREA and the 3' untranslated region of its mRNA. EMBO J. (1996). doi:10.1002/j.14602075.1996.tb00639.x

22. Berger, H. et al. The GATA factor AreA regulates localization and in vivo binding site occupancy of the nitrate activator NirA. Mol. Microbiol. (2006). doi:10.1111/j.1365-2958.2005.04957.x

23. Morozov, I. Y., Galbis-Martinez, M., Jones, M. G. \& Caddick, M. X. Characterization of nitrogen metabolite signalling in Aspergillus via the regulated degradation of are A mRNA. Mol. Microbiol. (2001). doi:10.1046/j.13652958.2001.02636.x

24. Andrianopoulos, A., Kourambas, S., Sharp, J. A., Davis, M. A. \& Hynes, M. J. Characterization of the Aspergillus nidulans nmrA gene involved in nitrogen metabolite repression. J. Bacteriol. (1998). 
25. Macios, M., Caddick, M. X., Weglenski, P., Scazzocchio, C. \& Dzikowska, A. The GATA factors AREA and AREB together with the co-repressor NMRA, negatively regulate arginine catabolism in Aspergillus nidulans in response to nitrogen and carbon source. Fungal Genet. Biol. (2012). doi:10.1016/j.fgb.2012.01.004

26. Berger, H. et al. Dissecting individual steps of nitrogen transcription factor cooperation in the Aspergillus nidulans nitrate cluster. Mol. Microbiol. (2008). doi:10.1111/j.1365-2958.2008.06359.x

27. Muro-Pastor, M. I. The GATA factor AreA is essential for chromatin remodelling in a eukaryotic bidirectional promoter. EMBO J. (1999). doi:10.1093/emboj/18.6.1584

28. Fu, Y. H. \& Marzluf, G. A. Characterization of nit-2, the major nitrogen regulatory gene of Neurospora crassa. Mol. Cell. Biol. (1987). doi:10.1128/mcb.7.5.1691

29. Feng, B. \& Marzluf, G. A. Interaction between Major Nitrogen Regulatory Protein NIT2 and Pathway-Specific Regulatory Factor NIT4 Is Required for Their Synergistic Activation of Gene Expression in Neurospora crassa . Mol. Cell. Biol. (1998). doi:10.1128/mcb.18.7.3983

30. Tudzynski, B., Homann, V., Feng, B. \& Marzluf, G. A. Isolation, characterization and disruption of the areA nitrogen regulatory gene of Gibberella fujikuroi. Mol. Gen. Genet. (1999). doi:10.1007/s004380050947

31. Arst, H. N. \& Cove, D. J. Nitrogen metabolite repression in Aspergillus nidulans. MGG Mol. Gen. Genet. (1973). doi:10.1007/BF00330988

32. Mo, X. \& Marzluf, G. A. Cooperative action of the NIT2 and NIT4 transcription factors upon gene expression in Neurospora crassa. Current Genetics (2003). doi:10.1007/s00294-002-0362-3

33. Bolton, M. D. \& Thomma, B. P. H. J. The complexity of nitrogen metabolism and nitrogen-regulated gene expression in plant pathogenic fungi. Physiological and Molecular Plant Pathology (2008). doi:10.1016/j.pmpp.2008.07.001

34. Divon, H. H., Ziv, C., Davydov, O., Yarden, O. \& Fluhr, R. The global nitrogen regulator, FNR1, regulates fungal nutrition-genes and fitness during Fusarium oxysporum pathogenesis. Mol. Plant Pathol. (2006). doi:10.1111/j.13643703.2006.00354.x

35. Pellier, A. L., Laugé, R., Veneault-Fourrey, C. \& Langin, T. CLNR1, the AREA/NIT2-like global nitrogen regulator of the plant fungal pathogen Colletotrichum lindemuthianum is required for the infection cycle. Mol. Microbiol. (2003). doi:10.1046/j.1365-2958.2003.03451.x

36. Pérez-García, A., Snoeijers, S. S., Joosten, M. H. A. J., Goosen, T. \& De Wit, P. J. G. M. Expression of the avirulence gene Avr9 of the fungal tomato pathogen 
Cladosporium fulvum is regulated by the global nitrogen response factor NRF1. Mol. Plant-Microbe Interact. (2001). doi:10.1094/MPMI.2001.14.3.316

37. Davis, M. A. \& Wong, K. H. Nitrogen Metabolism in Filamentous Fungi. in Cellular and Molecular Biology of Filamentous Fungi 325-338 (2014). doi:10.1128/9781555816636.ch23

38. Kmetzsch, L. et al. The GATA-type transcriptional activator Gat1 regulates nitrogen uptake and metabolism in the human pathogen Cryptococcus neoformans. Fungal Genet. Biol. (2011). doi:10.1016/j.fgb.2010.07.011

39. Jargeat, P., Gay, G., Debaud, J. C. \& Marmeisse, R. Transcription of a nitrate reductase gene isolated from the symbiotic basidiomycete fungus Hebeloma cylindrosporum does not require induction by nitrate. Mol. Gen. Genet. (2000). doi:10.1007/PL00008695

40. Martin, F., Cote, R. \& Canet, D. NH4+ assimilation in the ectomycorrhizal basidiomycete Laccaria bicolor (Maire) Orton, a 15N-NMR study. New Phytol. 128, 479-485 (1994).

41. Banuett, F. \& Herskowitz, I. Discrete developmental stages during teliospore formation in the corn smut fungus, Ustilago maydis. Development (1996).

42. León-Ramírez, C. G. et al. Infection of alternative host plant species by Ustilago maydis. New Phytol. (2004). doi:10.1111/j.1469-8137.2004.01171.x

43. Klosterman, S. J., Perlin, M. H., Garcia-Pedrajas, M., Covert, S. F. \& Gold, S. E. Genetics of Morphogenesis and Pathogenic Development of Ustilago maydis. Adv. Genet. 57, 1-47 (2007).

44. Basse, C. W. \& Steinberg, G. Ustilago maydis, model system for analysis of the molecular basis of fungal pathogenicity. Molecular Plant Pathology (2004). doi:10.1111/j.1364-3703.2004.00210.x

45. Feldbrügge, M., Kämper, J., Steinberg, G. \& Kahmann, R. Regulation of mating and pathogenic development in Ustilago maydis. Current Opinion in Microbiology (2004). doi:10.1016/j.mib.2004.10.006

46. Kämper, J. et al. Insights from the genome of the biotrophic fungal plant pathogen Ustilago maydis. Nature 444, 97-101 (2006).

47. Ho, E. C. H., Cahill, M. J. \& Saville, B. J. Gene discovery and transcript analyses in the corn smut pathogen Ustilago maydis: Expressed sequence tag and genome sequence comparison. BMC Genomics (2007). doi:10.1186/1471-2164-8-334

48. Aguilar, L. R. et al. Lipid droplets accumulation and other biochemical changes induced in the fungal pathogen Ustilago maydis under nitrogen-starvation. Arch. Microbiol. 199, 1195-1209 (2017).

49. Feldbrügge, M., Kellner, R. \& Schipper, K. The biotechnological use and potential 
of plant pathogenic smut fungi. Applied Microbiology and Biotechnology (2013). doi:10.1007/s00253-013-4777-1

50. Romero-Aguilar, L. et al. Rapamycin induces morphological and physiological changes without increase in lipid content in Ustilago maydis. Arch. Microbiol. 12, (2020).

51. Lewis, C. M. \& Fincham, J. R. Regulation of nitrate reductase in the basidiomycete Ustilago maydis. J. Bacteriol. (1970). doi:10.1128/jb.103.1.5561.1970

52. Ohashi, Y. et al. Regulation of nitrate assimilation in cyanobacteria. J. Exp. Bot. 62, 1411-1424 (2011).

53. Suzuki, I. et al. A novel nitrite reductase gene from the cyanobacterium Plectonema boryanum. J. Bacteriol. (1995). doi:10.1128/jb.177.21.61376143.1995

54. Siverio, J. M. Assimilation of nitrate by yeasts. FEMS Microbiology Reviews (2002). doi:10.1016/S0168-6445(02)00100-6

55. McCann, M. P. \& Snetselaar, K. M. A genome-based analysis of amino acid metabolism in the biotrophic plant pathogen Ustilago maydis. Fungal Genet. Biol. 45, 77-87 (2008).

56. Banks, G., Shelton, P., Kanuga, N., Holden, D. \& Spanos, A. The Ustilago maydis nar1 gene encoding nitrate reductase activity - sequence and transcriptional regulation.pdf. Gene 131, 69-78 (1993).

57. Kronstad, J. W. \& Staben, C. MATING TYPE IN FILAMENTOUS FUNGI. Annu. Rev. Genet. (1997). doi:10.1146/annurev.genet.31.1.245

58. Bölker, M., Genin, S., Lehmler, C. \& Kahmann, R. Genetic regulation of mating and dimorphism in Ustilago maydis . Can. J. Bot. (1995). doi:10.1139/b95-262

59. Gillissen, B. et al. A two-component regulatory system for self/non-self recognition in Ustilago maydis. Cell (1992). doi:10.1016/0092-8674(92)90141-X

60. Urban, M., Kahmann, R. \& Bölker, M. Identification of the pheromone response element in Ustilago maydis. Mol. Gen. Genet. (1996). doi:10.1007/s004380050136

61. Kämper, J., Reichmann, M., Romeis, T., Bölker, M. \& Kahmann, R. Multiallelic recognition: Nonself-dependent dimerization of the $\mathrm{bE}$ and $\mathrm{bW}$ homeodomain proteins in ustilago maydis. Cell (1995). doi:10.1016/0092-8674(95)90372-0

62. Asante-Owusu, R. N., Banham, A. H., Böhnert, H. U., Mellor, E. J. C. \& Casselton, L. A. Heterodimerization between two classes of homeodomain proteins in the mushroom Coprinus cinereus brings together potential DNAbinding and activation domains. Gene (1996). doi:10.1016/0378-1119(96)00177-1 
63. Hartmann, H. A., Kahmann, R. \& Bölker, M. The pheromone response factor coordinates filamentous growth and pathogenicity in Ustilago maydis. EMBO J. (1996). doi:10.1002/j.1460-2075.1996.tb00508.x

64. Kronstad, J. W. \& Leong, S. A. Isolation of two alleles of the b locus of Ustilago maydis. Proc. Natl. Acad. Sci. U. S. A. (1989). doi:10.1073/pnas.86.3.978

65. Paul, J. A., Wallen, R. M., Zhao, C., Shi, T. \& Perlin, M. H. Coordinate regulation of Ustilago maydis ammonium transporters and genes involved in mating and pathogenicity. Fungal Biol. (2018). doi:10.1016/j.funbio.2018.03.011

66. Brefort, T. et al. Ustilago maydis as a Pathogen . Annu. Rev. Phytopathol. 47, 423-445 (2009).

67. Pan, X., Harashima, T. \& Heitman, J. Signal transduction cascades regulating pseudohyphal differentiation of Saccharomyces cerevisiae. Current Opinion in Microbiology (2000). doi:10.1016/S1369-5274(00)00142-9

68. Kaffarnik, F., Müller, P., Leibundgut, M., Kahmann, R. \& Feldbrügge, M. PKA and MAPK phosphorylation of Prf1 allows promoter discrimination in Ustilago maydis. EMBO J. 22, 5817-5826 (2003).

69. Kahmann, R., Basse, C. \& Feldbrügge, M. Fungal-plant signalling in the Ustilago maydis-maize pathosystem. Current Opinion in Microbiology (1999). doi:10.1016/S1369-5274(99)00038-7

70. Krüger, J., Loubradou, G., Regenfelder, E., Hartmann, A. \& Kahmann, R. Crosstalk between cAMP and pheromone signalling pathways in Ustilago maydis. Mol. Gen. Genet. (1998). doi:10.1007/s004380050885

71. Gutkind, J. S. Regulation of mitogen-activated protein kinase signaling networks by G protein-coupled receptors. Science's STKE : signal transduction knowledge environment (2000). doi:10.1126/stke.2000.40.re1

72. Goldsmith, Z. G. \& Dhanasekaran, D. N. G Protein regulation of MAPK networks. Oncogene (2007). doi:10.1038/sj.onc.1210407

73. Müller, P., Aichinger, C., Feldbrügge, M. \& Kahmann, R. The MAP kinase Kpp2 regulates mating and pathogenic development in Ustilago maydis. Mol. Microbiol. (1999). doi:10.1046/j.1365-2958.1999.01661.x

74. Mayorga, M. E. \& Gold, S. E. A MAP kinase encoded by the ubc3 gene of Ustilago maydis is required for filamentous growth and full virulence. Mol. Microbiol. 34, 485-497 (1999).

75. Müller, P., Weinzierl, G., Brachmann, A., Feldbrügge, M. \& Kahmann, R. Mating and Pathogenic Development of the Smut Fungus Ustilago maydis Are Regulated by One Mitogen-Activated Protein Kinase Cascade. Eukaryot. Cell 2, 1187-1199 (2003). 
76. Kahmann, R. \& Kämper, J. Ustilago maydis: How its biology relates to pathogenic development. New Phytol. 164, 31-42 (2004).

77. Gold, S., Duncan, G., Barrett, K. \& Kronstad, J. cAMP regulates morphogenesis in the fungal pathogen Ustilago maydis. Genes Dev. (1994).

doi:10.1101/gad.8.23.2805

78. Gold, S. E., Brogdon, S. M., Mayorga, M. E. \& Kronstad, J. W. The Ustilago maydis regulatory subunit of a CAMP-dependent protein kinase is required for gall formation in maize. Plant Cell (1997). doi:10.1105/tpc.9.9.1585

79. Smith, D. G., Garcia-Pedrajas, M. D., Gold, S. E. \& Perlin, M. H. Isolation and characterization from pathogenic fungi of genes encoding ammonium permeases and their roles in dimorphism. Mol. Microbiol. 50, 259-275 (2003).

80. Lengeler, K. B. et al. Signal Transduction Cascades Regulating Fungal Development and Virulence. Microbiol. Mol. Biol. Rev. (2000). doi:10.1128/mmbr.64.4.746-785.2000

81. Stakman, E. C., Kernkamp, M. F., King, T. H. \& Martin, W. J. Genetic Factors for Mutability and Mutant Characters in Ustilago zeae. Am. J. Bot. (1943). doi:10.2307/2437392

82. Wallen, R. M. Regulation of haploid phenotypes in Ustilago maydis by ammonium transporters and components of the $\mathrm{b}$ mating locus. (University of Louisville, 2017). doi:https://doi.org/10.18297/etd/2771

83. Bölker, M. Ustilago maydis - A valuable model system for the study of fungal dimorphism and virulence. Microbiology (2001). doi:10.1099/00221287-147-61395

84. Glazebrook, J. Contrasting Mechanisms of Defense Against Biotrophic and Necrotrophic Pathogens. Annu. Rev. Phytopathol. 43, 205-227 (2005).

85. Banuett, F. Genetics of Ustilago maydis, A Fungal Pathogen that Induces Tumors in Maize. Annu. Rev. Genet. 29, 179-208 (1995).

86. Banuett, F. \& Herskowitz, I. Morphological Transitions in the Life Cycle of Ustilago maydis and Their Genetic Control by the a and b Loci. Exp. Mycol. (1994). doi:10.1006/emyc.1994.1024

87. Snoeijers, S. S., Pérez-García, A., Joosten, M. H. A. J. \& De Wit, P. J. G. M. The effect of nitrogen on disease development and gene expression in bacterial and fungal plant pathogens. European Journal of Plant Pathology (2000). doi:10.1023/A:1008720704105

88. Tavernier, V. et al. The plant nitrogen mobilization promoted by Colletotrichum lindemuthianum in Phaseolus leaves depends on fungus pathogenicity. J. Exp. Bot. (2007). doi:10.1093/jxb/erm182 
89. Hoffland, E., Jeger, M. J. \& Van Beusichem, M. L. Effect of nitrogen supply rate on disease resistance in tomato depends on the pathogen. Plant Soil (2000). doi:10.1023/A:1014960507981

90. Liao, J. et al. Pathogen effectors and plant immunity determine specialization of the blast fungus to rice subspecies. Elife (2016). doi:10.7554/elife.19377

91. Deller, S., Hammond-Kosack, K. E. \& Rudd, J. J. The complex interactions between host immunity and non-biotrophic fungal pathogens of wheat leaves. Journal of Plant Physiology (2011). doi:10.1016/j.jplph.2010.05.024

92. Hammond-Kosack, K. E. \& Parker, J. E. Deciphering plant-pathogen communication: Fresh perspectives for molecular resistance breeding. Current Opinion in Biotechnology (2003). doi:10.1016/S0958-1669(03)00035-1

93. Mur, L. A. J., Simpson, C., Kumari, A., Gupta, A. K. \& Gupta, K. J. Moving nitrogen to the centre of plant defence against pathogens. Annals of Botany (2017). doi:10.1093/aob/mcw179

94. Doehlemann, G. et al. Reprogramming a maize plant: Transcriptional and metabolic changes induced by the fungal biotroph Ustilago maydis. Plant J. 56, 181-195 (2008).

95. Horst, R. J. et al. Ustilago maydis infection strongly alters organic nitrogen allocation in maize and stimulates productivity of systemic source leaves. Plant Physiol. 152, 293-308 (2010).

96. Lovely, B. C., Aulakh, K. B. \& Perlin, M. H. Role of Hsl7 in morphology and pathogenicity and its interaction with other signaling components in the plant pathogen ustilago maydis. Eukaryot. Cell 10, 869-883 (2011).

97. Ruiz-Herrera, J. et al. A novel intracellular nitrogen-fixing symbiosis made by Ustilago maydis and Bacillus spp. New Phytol. 207, 769-777 (2015).

98. Holliday, R. Ustilago maydis. in Bacteria, Bacteriophages, and Fungi (1974). doi:10.1007/978-1-4899-1710-2_31

99. Cooper, M. Atmospheric nitrogen assimilation in Ustilago. (University of Louisville, 2015). doi:https://doi.org/10.18297/etd/2322

100. Pfannmüller, A., Boysen, J. M. \& Tudzynski, B. Nitrate assimilation in Fusarium fujikuroi is controlled by multiple levels of regulation. Front. Microbiol. 8, (2017).

101. Cove, D. J. the Induction and Repression of Nit Rate Red Uctase in the. BBA Enzymol. Biol. Oxid. 113, 51-56 (1965).

102. Holliday, R. The genetics of ustilago maydis. Genet. Res. (1961). doi:10.1017/S0016672300000719

103. Swamy, U. et al. Structure of spinach nitrite reductase: Implications for multi- 
electron reactions by the iron-sulfur:siroheme cofactor. Biochemistry (2005). doi:10.1021/bi050981y

104. Hall, N. \& Tomsett, A. B. Structure-function analysis of NADPH:nitrate reductase from Aspergillus nidulans: Analysis of altered pyridine nucleotide specificity in vivo. Microbiology (2000). doi:10.1099/00221287-146-6-1399

105. Campbell, W. H. \& Kinghorn, J. R. Functional domains of assimilatory nitrate reductases and nitrite reductases. Trends Biochem. Sci. (1990). doi:10.1016/09680004(90)90021-3

106. Rivas, J., Guerrero, M. G., Paneque, A. \& Losada, M. Characterization of the nitrate-reducing system of the yeast Torulopsis nitratophila. Plant Sci. Lett. (1973). doi:10.1016/0304-4211(73)90039-4

107. Prodouz, K. N. \& Garrett, R. H. Neurospora crassa NAD(P)H-nitrite reductase. Studies on its composition and structure. J. Biol. Chem. 256, 9711-9717 (1981).

108. Grigoriev, I. V. et al. Fueling the future with fungal genomics. Mycology (2011). doi:10.1080/21501203.2011.584577

109. Lu, S. et al. CDD/SPARCLE: the conserved domain database in 2020. Nucleic Acids Res. (2020). doi:10.1093/nar/gkz991

110. Hirasawa, T. et al. Comparative analysis of transcriptional responses to saline stress in the laboratory and brewing strains of Saccharomyces cerevisiae with DNA microarray. Appl. Microbiol. Biotechnol. (2006). doi:10.1007/s00253-0050192-6

111. Nikolaou, E. et al. Phylogenetic diversity of stress signalling pathways in fungi. BMC Evol. Biol. (2009). doi:10.1186/1471-2148-9-44

112. Rozen, S. \& Skaletsky, H. Primer3 on the WWW for General Users and for Biologist Programmers. in Bioinformatics Methods and Protocols (2000). doi:10.1385/1-59259-192-2:365

113. Brachmann, A., König, J., Julius, C. \& Feldbrügge, M. A reverse genetic approach for generating gene replacement mutants in Ustilago maydis. Mol. Genet. Genomics (2004). doi:10.1007/s00438-004-1047-z

114. Terfrüchte, M. et al. Establishing a versatile Golden Gate cloning system for genetic engineering in fungi. Fungal Genet. Biol. (2014). doi:10.1016/j.fgb.2013.10.012

115. Harrison, N., Cavinder, B., Townsend, J. P. \& Trail, F. Optimized primers and other critical conditions for efficient fusion PCR to generate knockout vectors in filamentous fungi. Fungal Genet. Rep. 60, 1-10 (2013).

116. Hallen-Adams, H. E., Cavinder, B. L. \& Trail, F. Fusarium graminearum from expression analysis to functional assays. Methods Mol. Biol. (2011). 
doi:10.1007/978-1-61779-040-9_6

117. Gibson, D. G. et al. Enzymatic assembly of DNA molecules up to several hundred kilobases. Nat. Methods (2009). doi:10.1038/nmeth.1318

118. Banuett, F. \& Herskowitz, I. Different a alleles of Ustilago maydis are necessary for maintenance of filamentous growth but not for meiosis. Proc. Natl. Acad. Sci. (1989). doi:10.1073/pnas.86.15.5878

119. Gooch, J. W. Kruskal-Wallis Test. in Encyclopedic Dictionary of Polymers (2011). doi:10.1007/978-1-4419-6247-8_15268

120. Kruskal, W. H. \& Wallis, W. A. Use of Ranks in One-Criterion Variance Analysis. J. Am. Stat. Assoc. (1952). doi:10.1080/01621459.1952.10483441

121. Haynes, W. Tukey's Test. in Encyclopedia of Systems Biology (eds. Dubitzky, W., Wolkenhauer, O., Cho, K.-H. \& Yokota, H.) 2303-2304 (Springer New York, 2013). doi:10.1007/978-1-4419-9863-7_1212

122. Altschul, S. F. et al. Gapped BLAST and PSI-BLAST: A new generation of protein database search programs. Nucleic Acids Research (1997). doi:10.1093/nar/25.17.3389

123. Lorenz, S. et al. Genome sequence of the basidiomycetous fungus Pseudozyma aphidis DSM70725, an efficient producer of biosurfactant mannosylerythritol lipids. Genome Announc. (2014). doi:10.1128/genomeA.e00053-14

124. Wortman, J. R. et al. The 2008 update of the Aspergillus nidulans genome annotation: a community effort. Fungal Genet. Biol. (2009). doi:10.1016/j.fgb.2008.12.003

125. Iida, Y., Kurata, T., Harimoto, Y. \& Tsuge, T. Nitrite reductase gene upregulated during conidiation is involved in macroconidium formation in Fusarium oxysporum. Phytopathology (2008). doi:10.1094/PHYTO-98-10-1099

126. Hartmann, A., Krüger, J., Lottspeich, F. \& Kahmann, R. Environmental signals controlling sexual development of the corn smut fungus Ustilago maydis through the transcriptional regulator Prf1. Plant Cell (1999). doi:10.1105/tpc.11.7.1293

127. Brachmann, A., Weinzierl, G., Kämper, J. \& Kahmann, R. Identification of genes in the bW/bE regulatory cascade in Ustilago maydis. Mol. Microbiol. (2001). doi:10.1046/j.1365-2958.2001.02699.x

128. Ray, P. et al. Scavenging organic nitrogen and remodelling lipid metabolism are key survival strategies adopted by the endophytic fungi, Serendipita vermifera and Serendipita bescii to alleviate nitrogen and phosphorous starvation in vitro. Environ. Microbiol. Rep. 11, 548-557 (2019).

129. Brito, N., Avila, J., Perez, M. D., Gonzalez, C. \& Siverio, J. M. The genes YNI1 and YNR1, encoding nitrite reductase and nitrate reductase respectively in the 
yeast Hansenula polymorpha, are clustered and coordinately regulated. Biochem. $J$. (1996). doi:10.1042/bj3170089

130. Gonzalez, C. et al. Nitrite causes reversible inactivation of nitrate reductase in the yeast Hansenula anomala. Microbiology (1994). doi:10.1099/00221287-140-102633

131. Li, J., Jia, W. \& Zhao, Q. Excessive nitrite affects zebrafish valvulogenesis through yielding too much NO signaling. PLoS One (2014). doi:10.1371/journal.pone.0092728

132. Ro \& Bson, S. Nitrate and nitrite poisoning in livestock. Primefacts (2007).

133. Li, X. Z. \& Oaks, A. The effect of light on the nitrate and nitrite reductases in Zea mays. Plant Sci. (1995). doi:10.1016/0168-9452(95)04159-R

134. Divon, H. H. \& Fluhr, R. Nutrition acquisition strategies during fungal infection of plants. FEMS Microbiology Letters (2007). doi:10.1111/j.1574-6968.2006.00504.x

135. Mendgen, K. \& Hahn, M. Plant infection and the establishment of fungal biotrophy. Trends in Plant Science (2002). doi:10.1016/S1360-1385(02)02297-5

136. Kim, S., Dale, B. E. \& Jenkins, R. Life cycle assessment of corn grain and corn stover in the United States. Int. J. Life Cycle Assess. (2009). doi:10.1007/s11367008-0054-4

137. Horowitz, S., Freeman, S., Zveibil, A. \& Yarden, O. A defect in nir1, a nirA-like transcription factor, confers morphological abnormalities and loss of pathogenicity in Colletotrichum acutatum. Mol. Plant Pathol. (2006). doi:10.1111/j.13643703.2006.00341.x

138. Heimel, K. et al. The transcription factor rbf 1 is the master regulator for b-mating type controlled pathogenic development in Ustilago maydis. PLoS Pathog. (2010). doi:10.1371/journal.ppat.1001035

139. Lanver, D. et al. Ustilago maydis effectors and their impact on virulence. Nat. Rev. Microbiol. 15, 409-421 (2017).

140. Scherer, M., Heimel, K., Starke, V. \& Kämper, J. The Clp1 protein is required for clamp formation and pathogenic development of Ustilago maydis. Plant Cell (2006). doi:10.1105/tpc.106.043521

141. Heimel, K., Scherer, M., Schuler, D. \& Kämper, J. The Ustilago maydis Clp1 protein orchestrates pheromone and $b$-dependent signaling pathways to coordinate the cell cycle and pathogenic development. Plant Cell (2010). doi: $10.1105 /$ tpc. 110.076265

142. Choudary, P. V. A high-throughput assay format for determination of nitrate reductase and nitrite reductase enzyme activities. Appl. Biochem. Biotechnol. Part A Enzym. Eng. Biotechnol. 62, 29-36 (1997). 
143. Crawford, N. \& Arst, H. N. The Molecular Genetics of Nitrate Assimilation in Fungi and Plants. Annu. Rev. Genet. 27, 115-146 (1993).

144. Pérez, M. D., González, C., Ávila, J., Brito, N. \& Siverio, J. M. The YNT1 gene encoding the nitrate transporter in the yeast Hansenula polymorpha is clustered with genes YNI1 and YNR1 encoding nitrite reductase and nitrate reductase, and its disruption causes inability to grow in nitrate. Biochem. J. (1997). doi:10.1042/bj3210397

145. Unkles, S. E. et al. crnA encodes a nitrate transporter in Aspergillus nidulans. Proc. Natl. Acad. Sci. U. S. A. (1991). doi:10.1073/pnas.88.1.204

146. Amaar, Y. G. \& Moore, M. M. Mapping of the nitrate-assimilation gene cluster (crnA-niiA-niaD) and characterization of the nitrite reductase gene (niiA) in the opportunistic fungal pathogen Aspergillus fumigatus. Curr. Genet. 33, 206-215 (1998).

147. Gao-Rubinelli, F. \& Marzluf, G. A. Identification and Characterization of a Nitrate Transporter Gene in Neurospora crassa. Biochem. Genet. (2004). doi:10.1023/B:BIGI.0000012141.51114.23

148. Jia, W., Tovell, N., Clegg, S., Trimmer, M. \& Cole, J. A single channel for nitrate uptake, nitrite export and nitrite uptake by Escherichia coli NarU and a role for NirC in nitrite export and uptake. Biochem. J. (2009). doi:10.1042/BJ20080746

149. Rowe, J. J., Ubbink-Kok, T., Molenaar, D., Konings, W. N. \& Driessen, A. J. M. Nark is a nitrite-extrusion system involved in anaerobic nitrate respiration by Escherichia coli. Mol. Microbiol. (1994). doi:10.1111/j.1365-2958.1994.tb01044.x

150. Sharma, V., Noriega, C. E. \& Rowe, J. J. Involvement of NarK1 and NarK2 proteins in transport of nitrate and nitrite in the denitrifying bacterium Pseudomonas aeruginosa PAO1. Appl. Environ. Microbiol. (2006). doi:10.1128/AEM.72.1.695-701.2006

151. Schuster, M., Schweizer, G. \& Kahmann, R. Comparative analyses of secreted proteins in plant pathogenic smut fungi and related basidiomycetes. Fungal Genet. Biol. (2018). doi:10.1016/j.fgb.2016.12.003

152. Heigwer, F., Kerr, G. \& Boutros, M. E-CRISP: Fast CRISPR target site identification. Nature Methods 11, 122-123 (2014).

153. Edgar, R. C. MUSCLE: A multiple sequence alignment method with reduced time and space complexity. BMC Bioinformatics (2004). doi:10.1186/1471-2105-5-113

154. Kumar, S., Stecher, G., Li, M., Knyaz, C. \& Tamura, K. MEGA X: Molecular evolutionary genetics analysis across computing platforms. Mol. Biol. Evol. (2018). doi:10.1093/molbev/msy096

155. Veenhoff, L. M., Heuberger, E. H. M. L. \& Poolman, B. Quaternary structure and function of transport proteins. Trends in Biochemical Sciences (2002). 
doi:10.1016/S0968-0004(02)02077-7

156. Donofrio, N. M. et al. Global gene expression during nitrogen starvation in the rice blast fungus, Magnaporthe grisea. Fungal Genet. Biol. (2006).

doi:10.1016/j.fgb.2006.03.005

157. Schuster, M., Schweizer, G., Reissmann, S. \& Kahmann, R. Genome editing in Ustilago maydis using the CRISPR-Cas system. Fungal Genet. Biol. (2016). doi:10.1016/j.fgb.2015.09.001

158. Garneau, J. E. et al. The CRISPR/cas bacterial immune system cleaves bacteriophage and plasmid DNA. Nature (2010). doi:10.1038/nature09523

159. Charpentier, E. \& Doudna, J. A. Rewriting a genome. Nature (2013). doi:10.1038/495050a

160. Wang, H., La Russa, M. \& Qi, L. S. CRISPR/Cas9 in Genome Editing and Beyond. Annu. Rev. Biochem. (2016). doi:10.1146/annurev-biochem-060815014607

161. Zuo, W., Depotter, J. R. \& Doehlemann, G. Cas9HF1 enhanced specificity in Ustilago maydis. Fungal Biol. (2020). doi:10.1016/j.funbio.2020.02.006

162. Takaya, N. Dissimilatory nitrate reduction metabolisms and their control in fungi. Journal of Bioscience and Bioengineering (2002). doi:10.1016/S13891723(02)80187-6

163. Roncero, C. \& Duran, A. Effect of Calcofluor White and Congo red on fungal cell wall morphogenesis: In vivo activation of chitin polymerization. J. Bacteriol. (1985). doi:10.1128/jb.163.3.1180-1185.1985

164. Apostol, I., Heinstein, P. F. \& Low, P. S. Rapid Stimulation of an Oxidative Burst during Elicitation of Cultured Plant Cells : Role in Defense and Signal Transduction. Plant Physiol. (1989). doi:10.1104/pp.90.1.109

165. Chen, L. H., Tsai, H. C., Yu, P. L. \& Chung, K. R. A major facilitator superfamily transporter- mediated resistance to oxidative stress and fungicides requires Yap1, Skn7, and MAP kinases in the citrus fungal pathogen alternaria alternata. PLoS One (2017). doi:10.1371/journal.pone.0169103

166. Gostinčar, C. \& Gunde-Cimerman, N. Overview of oxidative stress response genes in selected halophilic fungi. Genes (Basel). (2018). doi:10.3390/genes9030143

167. Follett, R. F. \& Delgado, J. A. Nitrogen fate and transport in agricultural systems. in Journal of Soil and Water Conservation (2002).

168. Farjad, M. et al. Nitrogen limitation alters the response of specific genes to biotic stress. Int. J. Mol. Sci. (2018). doi:10.3390/ijms19113364

169. Fedorova, E., Greenwood, J. S. \& Oaks, A. In-situ localization of nitrate reductase 
in maize roots. Planta (1994). doi:10.1007/BF01101689

170. Kim, H. \& Woloshuk, C. P. Role of AREA, a regulator of nitrogen metabolism, during colonization of maize kernels and fumonisin biosynthesis in Fusarium verticillioides. Fungal Genet. Biol. (2008). doi:10.1016/j.fgb.2008.03.007

171. Fagard, M. et al. Nitrogen metabolism meets phytopathology. Journal of Experimental Botany (2014). doi:10.1093/jxb/eru323

172. Musafia, B., Buchner, V. \& Arad, D. Complex salt bridges in proteins: Statistical analysis of structure and function. J. Mol. Biol. (1995).

doi:10.1006/jmbi.1995.0653

173. Sokalingam, S., Raghunathan, G., Soundrarajan, N. \& Lee, S. G. A study on the effect of surface lysine to arginine mutagenesis on protein stability and structure using green fluorescent protein. PLoS One (2012).

doi:10.1371/journal.pone.0040410

174. Kumar, S., Tsai, C. J. \& Nussinov, R. Factors enhancing protein thermostability. Protein Eng. (2000). doi:10.1093/protein/13.3.179

175. Azevedo, C. \& Saiardi, A. Why always lysine? The ongoing tale of one of the most modified amino acids. Advances in Biological Regulation (2016). doi:10.1016/j.jbior.2015.09.008

176. Isom, D. G., Castañed, C. A., Cannon, B. R. \& García-Moreno, B. E. Large shifts in $\mathrm{pKa}$ values of lysine residues buried inside a protein. Proc. Natl. Acad. Sci. U. S. A. (2011). doi:10.1073/pnas.1010750108

177. Bartlett, G. J., Porter, C. T., Borkakoti, N. \& Thornton, J. M. Analysis of catalytic residues in enzyme active sites. J. Mol. Biol. (2002). doi:10.1016/S00222836(02)01036-7

178. Davies, A. M. et al. Role of arginine-38 in regulation of the cytochrome c oxidation-reduction equilibrium. Biochemistry (1989). doi:10.1021/bi00434a012

179. Savage, D. F., O’Connell, J. D., Miercke, L. J. W., Finer-Moore, J. \& Stroud, R. M. Structural context shapes the aquaporin selectivity filter. Proc. Natl. Acad. Sci. U. S. A. (2010). doi:10.1073/pnas.1009864107

180. Harms, M. J., Schlessman, J. L., Sue, G. R. \& Bertrand García-Moreno, E. Arginine residues at internal positions in a protein are always charged. Proc. Natl. Acad. Sci. U. S. A. (2011). doi:10.1073/pnas.1104808108

181. Spellig, T., Bölker, M., Lottspeich, F., Frank, R. W. \& Kahmann, R. Pheromones trigger filamentous growth in Ustilago maydis. EMBO J. (1994). doi:10.1002/j.1460-2075.1994.tb06425.x

182. Snetselaar, K. M. \& Mims, C. W. Sporidial Fusion and Infection of Maize Seedlings by the Smut Fungus Ustilago maydis. Mycologia (1992). 
doi: $10.2307 / 3760250$

183. Spellig, T. et al. Control of mating and development in Ustilago maydis. Antonie Van Leeuwenhoek (1994). doi:10.1007/BF00871946

184. Banks, G. R., Shelton, P. A., Kanuga, N., Holden, D. W. \& Spanos, A. The Ustilago maydis nar 1 gene encoding nitrate reductase activity: sequence and transcriptional regulation. Gene (1993). doi:10.1016/0378-1119(93)90670-X

185. Jargeat, P. et al. Characterisation and expression analysis of a nitrate transporter and nitrite reductase genes, two members of a gene cluster for nitrate assimilation from the symbiotic basidiomycete Hebeloma cylindrosporum. Curr. Genet. (2003). doi:10.1007/s00294-003-0387-2

186. Unkles, S. E. et al. Nitrate reductase activity is required for nitrate uptake into fungal but not plant cells. J. Biol. Chem. (2004). doi:10.1074/jbc.M403974200

187. Ram, A. F. J. \& Klis, F. M. Identification of fungal cell wall mutants using susceptibility assays based on Calcofluor white and Congo red. Nat. Protoc. (2006). doi:10.1038/nprot.2006.397

188. Kopecká, M. \& Gabriel, M. The influence of Congo red on the cell wall and $(1 \rightarrow$ 3)- $\beta$-d-glucan microfibril biogenesis in Saccharomyces cerevisiae. Arch. Microbiol. (1992). doi:10.1007/BF00245214

189. Bastos, R. W. et al. Functional Characterization of Clinical Isolates of the Opportunistic Fungal Pathogen Aspergillus nidulans . mSphere (2020). doi:10.1128/msphere.00153-20

190. Kaeberlein, M., Andalis, A. A., Fink, G. R. \& Guarente, L. High Osmolarity Extends Life Span in Saccharomyces cerevisiae by a Mechanism Related to Calorie Restriction. Mol. Cell. Biol. (2002). doi:10.1128/mcb.22.22.80568066.2002

191. Murali, L. V. K. Exploring the role of PTN1 in ustilago maydis . (University of Louisville, 2019). doi:https://doi.org/10.18297/etd/3210

192. Wiemken, A. Trehalose in yeast, stress protectant rather than reserve carbohydrate. Antonie Van Leeuwenhoek (1990). doi:10.1007/BF00548935

193. Salmerón-Santiago, K. G. et al. Response to osmotic stress and temperature of the fungus Ustilago maydis. Archives of Microbiology (2011). doi:10.1007/s00203011-0706-9

194. Hohmann, S. Osmotic Stress Signaling and Osmoadaptation in Yeasts. Microbiol. Mol. Biol. Rev. (2002). doi:10.1128/mmbr.66.2.300-372.2002

195. Pham, C. D. et al. Ustilago maydis rho1 and 14-3-3 homologues participate in pathways controlling cell separation and cell polarity. Eukaryot. Cell (2009). doi:10.1128/EC.00009-09 
196. Lorenz, M. C. \& Heitman, J. The MEP2 ammonium permease regulates pseudohyphal differentiation in Saccharomyces cerevisiae. EMBO J. (1998). doi:10.1093/emboj/17.5.1236

197. Rutherford, J. C., Chua, G., Hughes, T., Cardenas, M. E. \& Heitman, J. A Mep2dependent transcriptional profile links permease function to gene expression during pseudohyphal growth in saccharomyces cerevisiae. Mol. Biol. Cell (2008). doi:10.1091/mbc.E08-01-0033

198. Gustin, M. C., Albertyn, J., Alexander, M. \& Davenport, K. MAP Kinase Pathways in the YeastSaccharomyces cerevisiae. Microbiol. Mol. Biol. Rev. (1998). doi:10.1128/mmbr.62.4.1264-1300.1998

199. Ruiz-Herrera, J., Pérez-Rodríguez, F. \& Velez-Haro, J. The signaling mechanisms involved in the dimorphic phenomenon of the Basidiomycota fungus Ustilago maydis. Int. Microbiol. 23, 121-126 (2020).

200. Paul, J. A., Barati, M. T., Cooper, M. \& Perlin, M. H. Physical and genetic interaction between Ammonium transporters and the signaling Protein Rho1 in the plant Pathogen Ustilago maydis. Eukaryot. Cell 13, 1328-1336 (2014).

201. Marzluf, G. A. Regulation of Nitrogen Metabolism in Mycelial Fungi. in Biochemistry and Molecular Biology 357-368 (1996). doi:10.1007/978-3-66210367-8_16

202. Davis, M. A. \& Wong, K. H. Nitrogen Metabolism in Filamentous Fungi. in Cellular and Molecular Biology of Filamentous Fungi 325-338 (American Society of Microbiology, 2014). doi:10.1128/9781555816636.ch23

203. Caddick, M. X. Nitrogen Regulation in Mycelial Fungi. in Biochemistry and Molecular Biology (2004). doi:10.1007/978-3-662-06064-3_17

204. Caddick, M. X., Peters, D. \& Platt, A. Nitrogen regulation in fungi. Antonie Van Leeuwenhoek (1994). doi:10.1007/BF00871943

205. Santi, S., Locci, G., Monte, R., Pinton, R. \& Varanini, Z. Induction of nitrate uptake in maize roots: Expression of a putative high-affinity nitrate transporter and plasma membrane H+-ATPase isoforms. J. Exp. Bot. (2003). doi:10.1093/jxb/erg208

206. Abrahamian, M., Ah-Fong, A. M. V., Davis, C., Andreeva, K. \& Judelson, H. S. Gene Expression and Silencing Studies in Phytophthora infestans Reveal Infection-Specific Nutrient Transporters and a Role for the Nitrate Reductase Pathway in Plant Pathogenesis. PLoS Pathog. (2016). doi:10.1371/journal.ppat.1006097

207. Solomon, P. S., Lowe, R. G. T., Tan, K. C., Waters, O. D. C. \& Oliver, R. P. Stagonospora nodorum: Cause of stagonospora nodorum blotch of wheat. Molecular Plant Pathology (2006). doi:10.1111/j.1364-3703.2006.00326.x 
208. Pataky, J. K. \& Snetselaar, K. M. Common smut of corn. Plant Heal. Instr. (2006). doi:10.1094/phi-i-2006-0927-01

209. Elías-Villalobos, A., Fernández-Álvarez, A., Moreno-Sánchez, I., Helmlinger, D. \& Ibeas, J. I. The Hos2 Histone Deacetylase Controls Ustilago maydis Virulence through Direct Regulation of Mating-Type Genes. PLoS Pathogens 11, (2015).

210. Daniel-Vedele, F., Filleur, S. \& Caboche, M. Nitrate transport: A key step in nitrate assimilation. Curr. Opin. Plant Biol. (1998). doi:10.1016/S13695266(98)80110-6

211. Shitan, N. \& Yazaki, K. New insights into the transport mechanisms in plant vacuoles. in International Review of Cell and Molecular Biology (2013). doi:10.1016/B978-0-12-407695-2.00009-3

212. Vyn, T. J., Janovicek, K. J., Miller, M. H. \& Beauchamp, E. G. Soil nitrate accumulation and corn response to preceding small-grain fertilization and cover crops. Agron. J. (1999). doi:10.2134/agronj1999.00021962009100010004x

213. Lazof, D. B., Rufty, T. W. \& Redinbaugh, M. G. Localization of nitrate absorption and translocation within morphological regions of the corn root. Plant Physiol. (1992). doi:10.1104/pp.100.3.1251

214. Wang, Y. et al. Nitrite transport is mediated by the nitrite-specific high-affinity NitA transporter and by nitrate transporters NrtA, NrtB in Aspergillus nidulans. Fungal Genet. Biol. (2008). doi:10.1016/j.fgb.2007.10.001

215. Gomez-Gil, L. et al. Nitrate assimilation pathway (NAP): role of structural (nit) and transporter (ntr1) genes in Fusarium oxysporum f.sp. lycopersici growth and pathogenicity. Curr. Genet. (2018). doi:10.1007/s00294-017-0766-8

216. Johnstone, I. L. et al. Isolation and characterisation of the crnA-niiA-niaD gene cluster for nitrate assimilation in Aspergillus nidulans. Gene (1990). doi:10.1016/0378-1119(90)90178-T

217. Cutler, S. B., Cooley, R. N. \& Caten, C. E. Cloning of the nitrate reductase gene of Stagonospora (Septoria) nodorum and its use as a selectable marker for targeted transformation. Curr. Genet. (1998). doi:10.1007/s002940050377

218. Perkins, D. D., Radford, A., Newmeyer, D. \& Bjorkman, M. Chromosomal loci of Neurospora crassa. Microbiological Reviews (1982). doi:10.1128/mmbr.46.4.426570.1982

219. Lanver, D. et al. The biotrophic development of ustilago maydis studied by RNAseq analysis. Plant Cell (2018). doi:10.1105/tpc.17.00764

220. Hirasawa, M. et al. Enzymatic properties of the ferredoxin-dependent nitrite reductase from chlamydomonas reinhardtii. Evidence for hydroxylamine as a late intermediate in ammonia production. Photosynth. Res. (2010). doi:10.1007/s11120-009-9512-5 
221. Lorenz, M. C. \& Heitman, J. Yeast pseudohyphal growth is regulated by GPA2, a G protein alpha homolog. EMBO J. (1997). doi:10.1093/emboj/16.23.7008

222. Kuppireddy, V. S. Identification and functional characterization of effectors from an anther smut fungus, Microbotryum lychnidis-dioicae . (University of Louisville, 2018). doi:https://doi.org/10.18297/etd/3078

223. He, F. Laemmli-SDS-PAGE. BIO-PROTOCOL (2011). doi:10.21769/bioprotoc.80

224. Sds-page, L. \& Sds, T. Laemmli buffer Background. Nature (1970).

225. Paneque, A., Del Campo, F. F. \& Losada, M. Nitrite reduction by isolated chloroplasts in light. Nature (1963). doi:10.1038/198090b0

226. Böer, E., Schröter, A., Bode, R., Piontek, M. \& Kunze, G. Characterization and expression analysis of a gene cluster for nitrate assimilation from the yeast Arxula adeninivorans. Yeast (2009). doi:10.1002/yea.1653

227. Garrett, R. H. \& Amy, N. K. Nitrate Assimilation in Fungi. Adv. Microb. Physiol. (1979). doi:10.1016/S0065-2911(08)60414-2

228. Sengupta, S., Shaila, M. S. \& Rao, G. R. Purification and characterization of assimilatory nitrite reductase from Candida utilis. Biochem. J. (1996). doi:10.1042/bj3170147

229. Sengupta, S., Shaila, M. S. \& Rao, G. R. A novel autophosphorylation mediated regulation of nitrite reductase in Candida utilis. FEBS Lett. (1997). doi:10.1016/S0014-5793(97)01166-6

230. Simon, J. \& Klotz, M. G. Diversity and evolution of bioenergetic systems involved in microbial nitrogen compound transformations. Biochim. Biophys. Acta Bioenerg. (2013). doi:10.1016/j.bbabio.2012.07.005

231. Nelson, M. B., Martiny, A. C. \& Martiny, J. B. H. Global biogeography of microbial nitrogen-cycling traits in soil. Proc. Natl. Acad. Sci. U. S. A. (2016). doi:10.1073/pnas.1601070113

232. Hayatsu, M., Tago, K. \& Saito, M. Various players in the nitrogen cycle: Diversity and functions of the microorganisms involved in nitrification and denitrification. Soil Science and Plant Nutrition (2008). doi:10.1111/j.1747-0765.2007.00195.x 


\section{APPENDICES}

\section{APPENDIX I}

\section{ROLE OF UM03848 GENE IN FB1 AND FB2 STRAINS TO THE VIRULENCE}

OF U. MAYDIS

\section{Summary}

This study was conducted to understand the role of Ustilago maydis nitrite reductase gene, $u m 03848$, in the virulence to its host plant. The um03848 gene was initially disrupted in mating compatible haploid strains FB1 and FB2. The phenotypes of the $u m 03848$ deletion mutants relating to mating efficiency, pathogenesis and expression of genes in the mating and pathogenesis programs were examined. The result for the mating assay showed that the deletion of um03848 in $U$. maydis did not affect mating with its compatible partner, as assessed by " $f u z z$ " in the charcoal mating assay. However, deletion of um03848 in FB2 background significantly affected virulence in maize. Quantitative real-time PCR was used to examine expression of the ump2 (high-affinity ammonium transporter) gene and genes involved in the mating program. The mutants were grown in low and high ammonium and compared with their respective wild type progenitor strains grown under the same conditions. No observed differences were found for the of prfl (transcription factor for $\mathrm{a}$ and $\mathrm{b}$ genes) and $r b f l$ (the master regulator of $\mathrm{b}$ responsive gene expression). However, changes in the mutants included significant downregulation in $b W$ in FB1 $\Delta u m 03848$ on low ammonium, while these were 
upregulated in the FB2 mutants. Also, $m f a$ (encoding pheromone precursor) and $b E$ was downregulated in FB2 $\Delta u m 03848$ but upregulated in FB1 $\Delta u m 03848$. In contrast, ump2 expression was significantly upregulated in all the mutants in low ammonium. Thus, this study suggested that the um03848 affects the pathogenesis of $U$. maydis on its host plant in a FB2 background-dependent manner.

Even though, this report was presented at the $30^{\text {th }}$ Fungal Genetics Conference in March 2019, this is not being formally reported in this dissertation. This is because when the wildtype compatible strains FB1 and FB2 were crossed together and injected into host plants, the maize plants did not show the severity of symptoms as previously reported in published journal articles.

\section{Methods}

The $U$. maydis strains used in this study are listed in Table A.1 below. Strains were grown at $27^{\circ} \mathrm{C}$ in solid or liquid YEPS. Mating assay was done by co-spotting premixed compatible haploid strains in solid PDA with $1 \%$ activated charcoal plate as mentioned previously in Chapter II. For plant infection assay, strains grown in $20 \mathrm{ml}$ YEPS media up to $\mathrm{OD}_{600}$ of 1.0 were collected and resuspended in water to concentrate up to $\mathrm{OD}_{600}$ 3.0. The 8 days old Golden Bantam corn seedlings [Organic Golden Bantam, Territorial Seed Company] was injected with pre-mixed haploid strains of opposite mating type. The pathogenicity symptoms were measured from $0-5$ disease index ${ }^{78}$ as mentioned before in Chapter II. Statistical analysis of the disease index measures was performed using a Kruskal-Wallis Test with Multiple Comparison Test using R program ${ }^{119,120}$. RNA isolation from $U$. maydis strains and quantitative real-time PCR were carried out as described in ${ }^{65}$ and it is explained in Chapter II. 
Table A. 1 List of strains used in Appendix I

\begin{tabular}{lll}
\hline Ustilago Strain & Genotype & Reference \\
\hline FB1 & $a 1$ (mfal pral) b1 $(b E 1 b W 1)$ & Banuett and \\
FB2 & $a 2(m f a 2$ pra2) $b 2(b E 2 b W 2)$ & Herskowitz, 1989 \\
FB1 $\Delta u m 03848$ & $a 1 b 1$ um03848::hyg ${ }^{R}$ & This study \\
FB2 $\Delta u m 03848$ & $a 2 b 2$ um03848::hyg ${ }^{R}$ & This study \\
\hline
\end{tabular}

\section{Results}

The mating assay was performed on PDA charcoal medium for um03848 deleted FB1 and FB2 mutants along with mating compatible wild type haploids (FB1 X FB2). Equal mixtures of all these compatible haploid strains were pre-mixed and spotted on to plate and were grown for $24 \mathrm{hrs}$. The compatible mating strains of $\Delta u m 03848$ showed similar dikaryotic white "fuzz" as of wild type strains as indicated by red boxes in Figure A.1 A. This depicts that the deletion of $u m 03848$ in FB1 and FB2 background doesn't affect the mating efficiency of $U$. maydis.

To determine the effects of these mutants on virulence to the host plant, the opposite haploid strains grown overnight were pre-mixed and injected into 8 days old maize seedlings. Disease symptoms were scored at 14 days post infection (dpi) with the infection ranking of 0 to 5 as mentioned earlier. Figure A.1 B displays the percentage of plants with specific symptoms of infection. The infections caused by the pre-mixed compatible haploid strains of $\Delta u m 03848$ showed significantly reduced virulence compared to the wild type strains. The $\Delta u m 03848$ strains were combined with the wild type strain of opposite mating-type and compared with infections due to either both wild type and both mutant pairings. The combinations FB1 WT X FB2 $\triangle u m 03848$ and FB1 
$\triangle \mathrm{um} 03848$ X FB2 WT were more virulent when compared to $\triangle u m 03848$ (FB1 X FB2)

but less when compared to the FB1 WT X FB2 WT (Figure A.1B). Similar results were observed with independently constructed um03848 deletion mutant in FB2 (FB2

$\Delta u m 03848-2)$. Therefore, figure A.1B indicated that the deletion of um03848 gene in FB2 background significantly reduce the plant virulence.

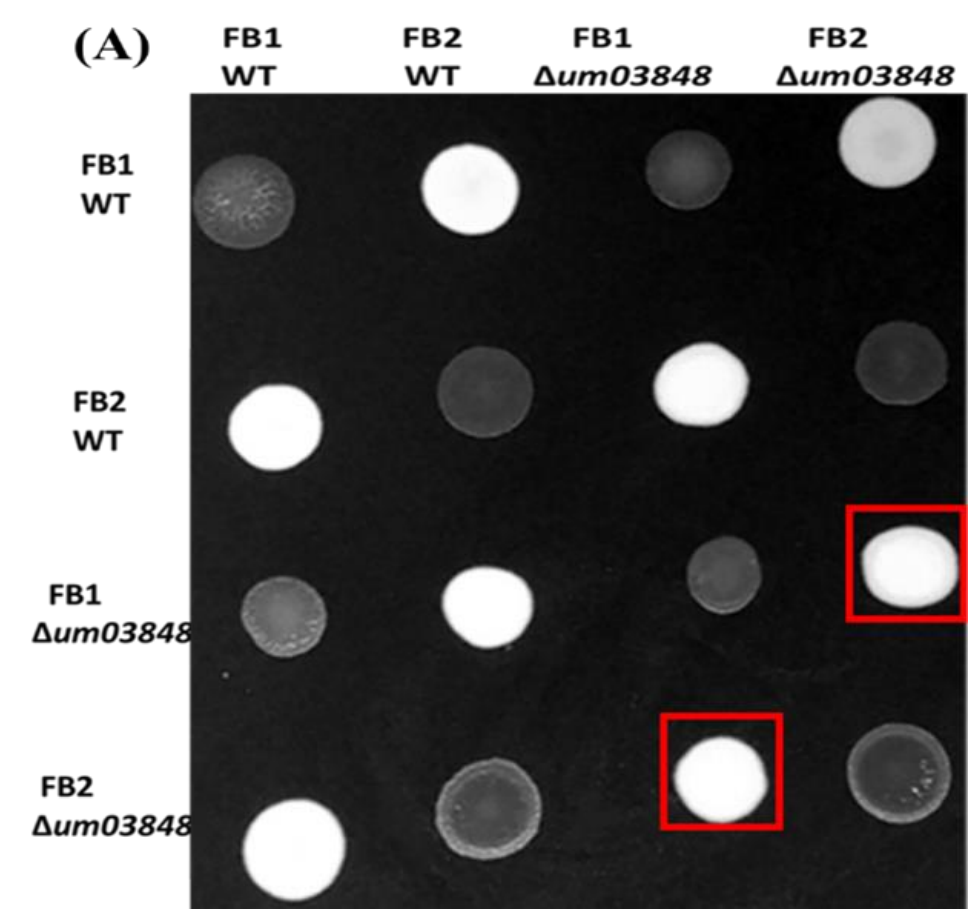




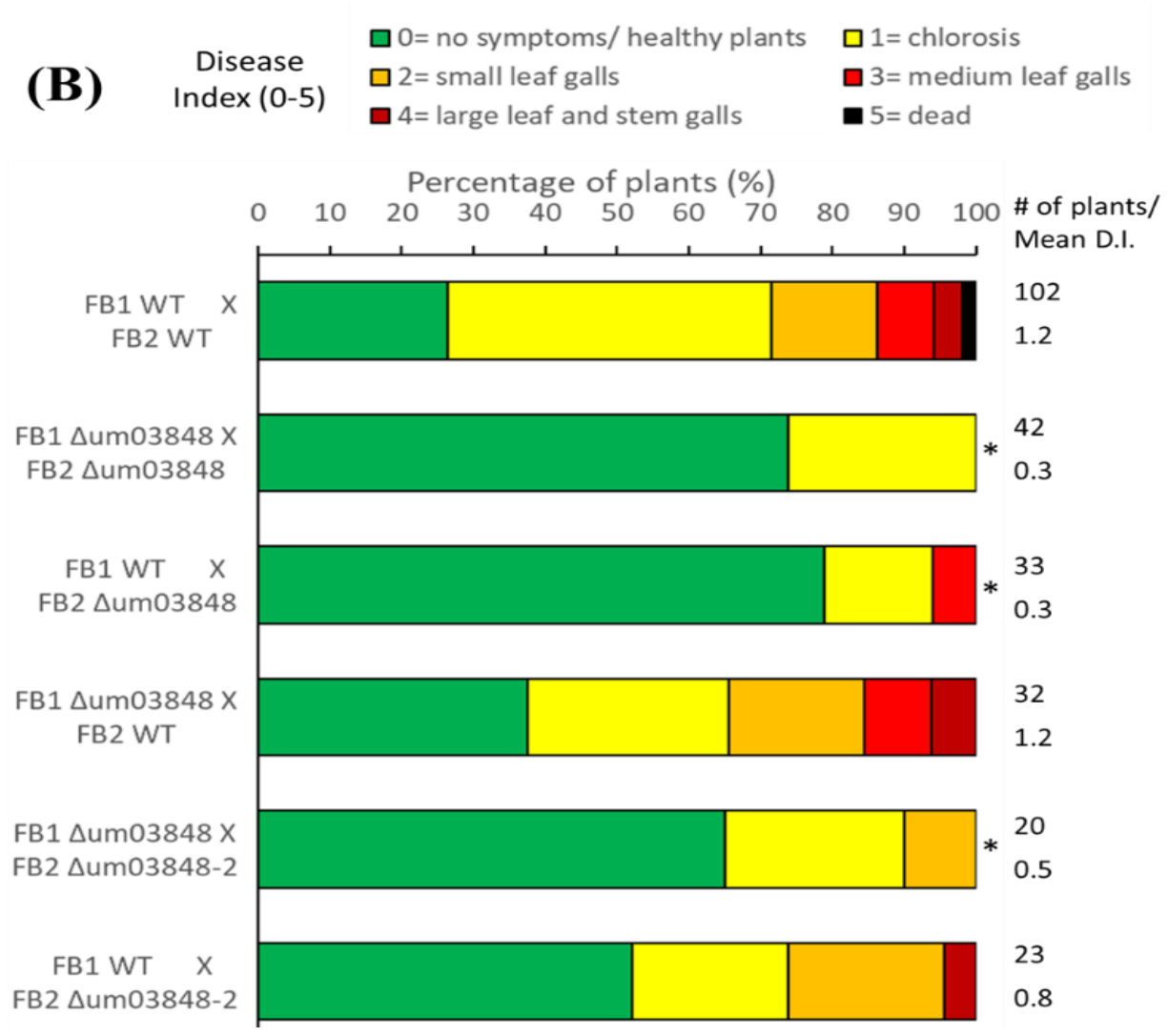

Figure A. 1 Phenotype changes related to deletion of um03848 on FB1 and FB2 strains. (A) Mating assay on PDA charcoal medium where equal mixtures of opposite mating wild type strains (FB1 WT X FB2 WT), corresponding um03848 deleted mutants (FB1 $\Delta u m 03848$ X FB2 $\Delta u m 03848$ ) and the mutants with the opposite wild type strains (WT X $\Delta u m 03848$ ) were spotted. After $24 \mathrm{~h}$ incubation, mating reaction was examined by the presence of white "fuzz" phenotype of aerial hyphae production. Red boxes indicate the successful mating of $\triangle u m 03848$ mating partner. (B) Pathogenicity assay where disease symptoms were scored at $14 \mathrm{dpi}$ in the categories of 0 to 5 as depicted at the top of the figure. Graph shows the percentage of plants with specific symptoms of infection. The Xaxis indicates the paired background that is injected into maize plant. The data was analyzed using Kruskal-Wallis test followed by post-hoc comparison and an asterisk (*) indicates significant difference $(\mathrm{p}<0.05)$ compared to those where both partners were wild type strains (FB1WT X FB2 WT). The significant reduction ( $\mathrm{p}<0.05)$ of plant pathogenesis was seen in um03848 deletion ( $\triangle u m 03848$ ) strain. Also, the virulence by FB2 $\triangle$ um03848 combined with FB1 WT was significantly lowered as compared to WT strains. Similar results were observed for the independently constructed mutant FB2 $\Delta$ um03848-2.

We observed that there is no effect of $\Delta u m 03848$ in mating efficiency of $U$.

maydis. However, there is significant decrease in the pathogenicity to the maize plant and 
this decrease in virulence is based on FB2 specific background. So, in order to analyze the difference in the mutants in different backgrounds, we performed qRT-PCR on mutants and wild type strains to observe the expression of the genes involved in mating program and high affinity ammonium transceptor, ump2. For this, the RNA extraction was done in the mutants and WT grown on low $(50 \mu \mathrm{M} \mathrm{NH} 4)$ and high $\left(30 \mathrm{mM} \mathrm{NH}_{4}\right)$ ammonium medium for $48 \mathrm{~h}$ as mentioned previously. qRT-PCR were performed using specific primers targeting genes involved in mating. Changes in the gene expression for the averages of three biological replicates, in relative to their respective wild type grown in high ammonium, are displayed as $\log 2$-fold changes in figure 2 . The genes on mating program were differentially regulated in the mutant strains (Figure $2 \mathrm{~A}$ ). The genes $m f a$ and $b E$ are significantly downregulation in FB2 backgrounds than FB1 background. The major difference was that the gene $b W$ was significantly downregulated in FB1 $\Delta u m 03848$ while upregulated in FB2 $\Delta u m 03848$ while comparing with their respective wild type strains grown in similar low ammonium medium. The high-affinity ammonium transceptor, $u m p 2$, was significantly upregulated in the $u m 03848$ deleted mutant in both backgrounds (FB1 and FB2) strains when compared to their respective WT in high ammonium (Figure 2B) whereas there was not significant difference with the WT grown in low ammonium medium.

Wallen 2017 found that ump2 senses nitrogen availability and upregulates the expression of mating targets in a $b$-dependent manner. She also suggested that the $b W$ regulates the transcription of mating and pathogenicity targets in a similar manner to ump2 i.e. induction of $p r f 1$ and $m f a$ genes in low ammonium. Therefore, significant upregulation of $b W$ in FB2 mutant (Figure 2A) should supposedly increase the 
pathogenicity of the fungus. However, FB2 background dependent reduction in plant virulence for um03848 gene (Figure 1B) couldn't be explained by the mating targets and ump2 gene expression.

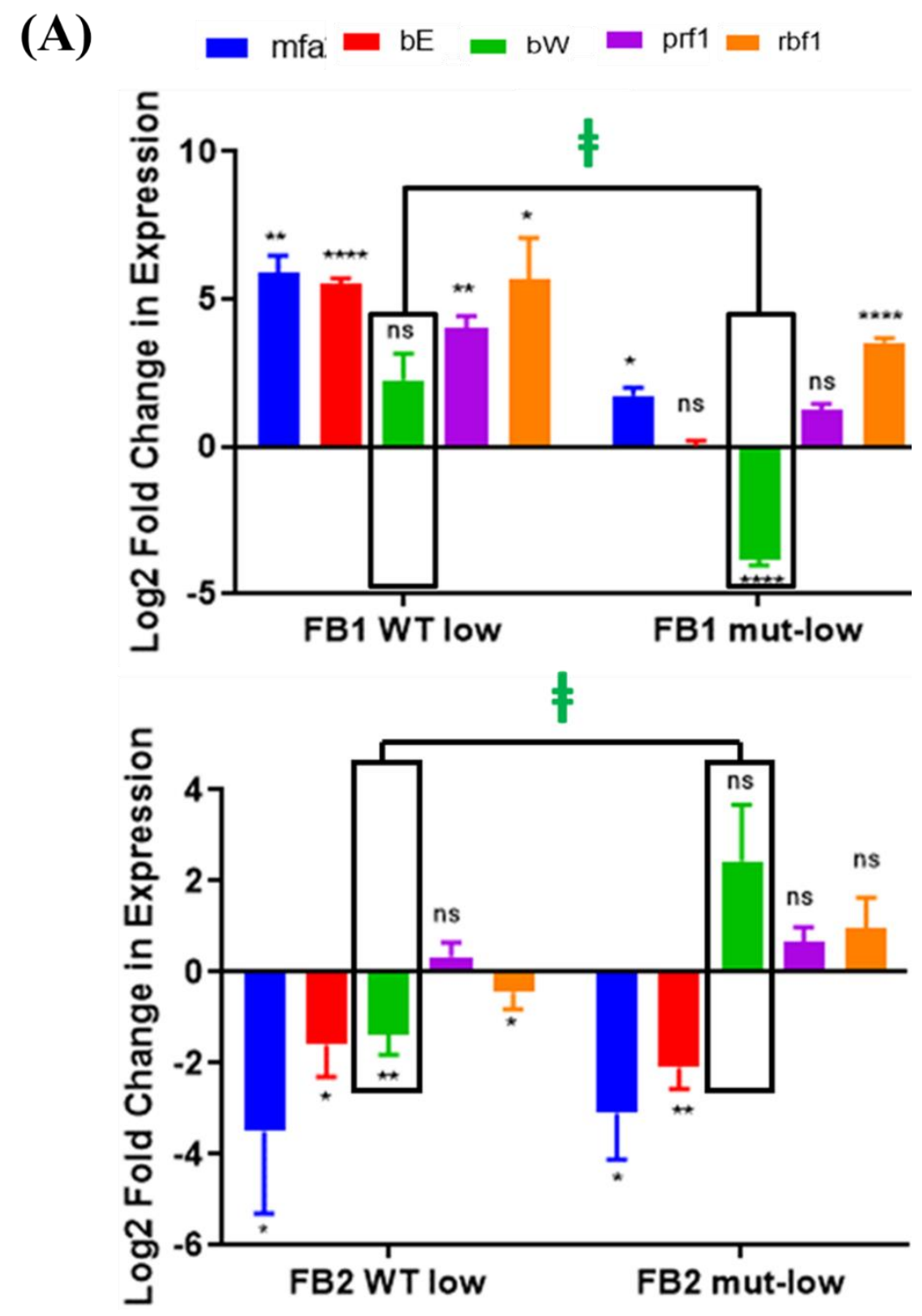




\section{(B) $u m p 2$}

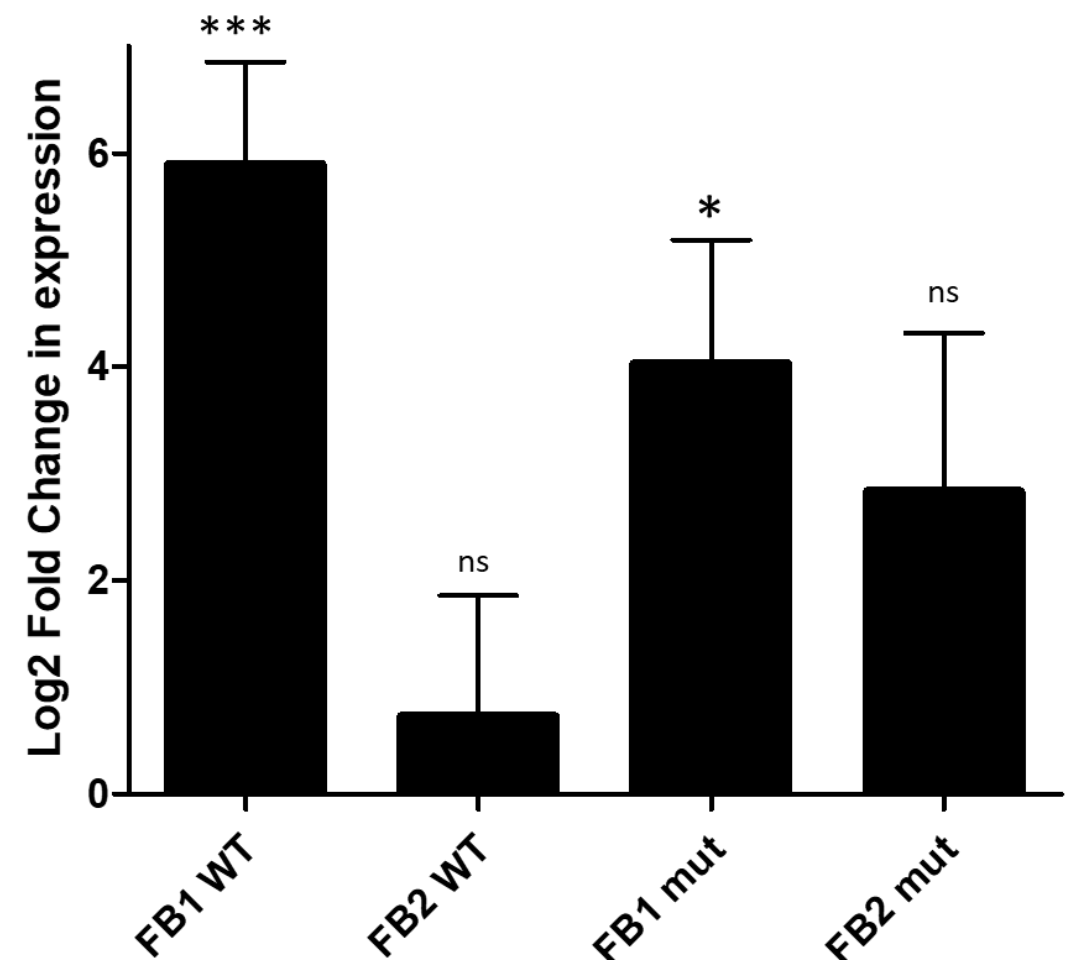

Figure A. 2 Log2 Fold changes in expression of mating targets and ump2 on wild type and mutant strains on low ammonium medium.

(A) genes involved in mating programs (B) high affinity ammonium transceptor gene, ump2. qRT-PCR for the wild type and um03848 deleted mutant strains grown on low ammonium medium was performed and normalized against eif 2 and expression measured in relative to the respective wild type grown on high ammonium medium. Negative values reflect the decrease in expression, whereas positive values represent increase in expression. Bar represents the averages of three biological replicates and standard errors are indicated by the line of top of bars in the graphs. All statistical analyses were done using Student's t-test (Unpaired two-tailed) in GraphPad Prism 7.0 in relative to wild type grown on high ammonium medium. $\mathrm{P}$ value $<0.05$ was considered significant. Statistical significance at $\mathrm{P}<0.05, \mathrm{P}<0.01$ and $\mathrm{P}<0.001$ are indicated as $*, * *, * * *$, respectively. $¥$ indicates the statistical significance ( $\mathrm{p}$-value $<0.05$ ) when deleted mutant is compared with their respective wild type strain, both grown in low ammonium medium. 


\section{APPENDIX II}

\section{BIOCHEMICAL ASSAY FOR U. MAYDIS' NITRITE REDUCTASE, UM03848}

\section{Introduction}

Nitrite reductase in fungi and bacteria uses $\mathrm{NAD}(\mathrm{P}) \mathrm{H}$ as an electron donor whereas in plants and algae, the enzyme uses ferredoxin. Generally, the protein has two prosthetic groups: iron-sulfur center and sirohaem group. In addition to these groups, bacteria and fungi possess $\mathrm{FAD}^{103,105,220}$. In chapter II, we observed that putative nitrite reductase gene in $U$. maydis, um03848, has sequence similarity to the known nitrite reductase of other filamentous fungi. The predicted um03848 protein consists of 602 amino acids residues and has the computed isotopically averaged molecular weight (https://web.expasy.org/compute_pi/) of 66457.67 (66.46 kDa). From the conserved domain analysis of this predicted amino acids, the um03848 amino acid sequence from positions 1 to 387 is identical to the entire sequence of nitrite reductase subunit, NirD from other proteobacteria. Within this sequence (amino acids from 35 to 87 ) is second bacterioferritin-associated ferredoxin (BFD)-like [2Fe-2S]-binding domain of the large subunit NADH-dependent nitrite reductase. The C-terminal portion of um03848 protein (amino acids 411 to 525) is similar Rieske domain of assimilatory nitrite reductase (NirD) family from other bacteria and fungi. The bacterial NirD contains a single Rieske domain while fungal proteins have a C-terminal Rieske domain. Rieske domain is a [ $2 \mathrm{Fe}-$ $2 S$ ] cluster binding domain involved in electron transfer and it is liganded to two histidine

and two cysteine residues present in conserved sequences called Rieske motifs ${ }^{109}$. Therefore, um03848 has the conserved domains of Rieske-like, Ferredoxin and 
$\mathrm{NAD}(\mathrm{P}) \mathrm{H}-\mathrm{NirD}$, which are the indicators of functional nitrite reductase. So, we wanted to confirm the nitrite reductase activity of um03848 and we analyzed the biochemical property of um03848 protein in this study.

\section{Methods}

Strains and growth conditions. The yeast strains used in this study are listed in Table A.2. Escherichia coli strain DH5 $\alpha$ (Thermo Fisher) was used for all cloning and plasmid maintenance. Yeast MLY40 $\alpha$ was grown on selective dropout (Ura-) medium at $30{ }^{\circ} \mathrm{C} . E$. coli cells were grown in LB medium supplemented with $200 \mu \mathrm{g} / \mathrm{mL}$ ampicillin or 50 $\mu \mathrm{g} / \mathrm{mL}$ kanamycin at $37^{\circ} \mathrm{C}$.

The induction of um03848 gene in transformed yeast (MLY40 $\alpha+p Y E S 2.1+3848$ ) was carried out by combining protocols from Haber Lab (http://www.bio.brandeis.edu/haberlab/jehsite/pdfs/GALInduc.pdf) and Buratowski Lab (http://tfiib.med.harvard.edu/Yeast_Galactose_Induction). Transformed yeast was grown in $4 \mathrm{~mL}$ liquid YPD medium containing $1 \%$ yeast extract, $2 \%$ peptone, $0.1 \%$ dextrose and $2 \%$ raffinose. Raffinose is added because glucose actively represses the Gal4 promoter and takes a while to switch back on whereas with raffinose Gal4 is not repressed and is quickly activated by addition of Galactose. Also, the yeast merely grew with just raffinose so glucose was added. The $2 \mathrm{~mL}$ of 2 days incubated yeast cells was used as inoculum for $50 \mathrm{ml}$ liquid YPD in $500 \mathrm{~mL}$ Erlenmeyer flask, providing enough aeration. The flask was vigorously shaken overnight or until $\mathrm{OD}_{600}$ of $0.6-0.8$ is reached. Yeasts are then induced by adding $1 / 10$ volume of $20 \%(\mathrm{w} / \mathrm{v})$ galactose $(2 \%$ final 
concentration) and continued shaking for 4 hours at $30^{\circ} \mathrm{C}$. Galactose was not added to one flask for an uninduced control.

Table A. 2 Strains used in Appendix II.

\begin{tabular}{ll}
\hline Yeast Strain & References \\
\hline MLY40 $\alpha$ Ura & Lorenz and Heitman, 1997 \\
MLY40 $\alpha+$ pYES 2.1 & This study \\
MLY40 $\alpha+$ pYES $2.1+3848$ & This study \\
\hline Plasmid & Reference \\
\hline$p$ TOPO+mcherry & Kuppireddy, 2018 \\
\hline pTOPO+3848 & This study
\end{tabular}

Vector construction and cloning. The primers listed on table A.3 were used to obtain the fragments containing um03848 gene to construct a vector. The first two primers listed on the table were used to amplify um03848 gene with its terminator from $U$. maydis genome. The fragment was inserted into pYES2.1TOPO vector and was cloned into DH5a E. coli cells. The colonies grown on LB plates with $100 \mathrm{mg} / \mathrm{L}$ ampicillin after overnight incubation at $37^{\circ} \mathrm{C}$ were PCR screened for presence of um03848 gene. The cloned E. coli should express um03848 under Gal1 promoter. Therefore, correct orientation of incorporation of $u m 03848$ was determined with further PCR screening followed by sequencing. The plasmid, pYES2 $1+3848$, from confirmed colony was used to transform into uracil-deficient (ura') S. cerevisiae strain (MLY40 $\left.\alpha \mathrm{Ura}^{-}\right)^{221}$. The transformation was performed by using Frozen-EZ Yeast Transformation II kit by ZymoResearch. As pYES2.1TOPO vector has Ura gene to utilize uracil, the successful transformants will be able to grow on an uracil incorporated plate. The colonies for successful cloning was tested and confirmed by PCR and sequencing. The $S$. cerevisiae 
strain (MLY40 $\alpha \mathrm{Ura}^{-}$) was also transformed with pYES2.1TOPO vector without um03848 gene for the control test.

To express $u m 03848$ in $E$. coli, TOPO+mcherry vector ${ }^{222}$ was used. The vector was digested with restriction enzyme EcoRI which removes mcherry out of the vector. The linearized vector was separated by gel electrophoresis where fragment was excised from the gel and purified using Zymoclean Gel DNA recovery kit. The purified linearized vector was cloned with PCR amplified um03848 gene (using TOPO primers, table A.3) from $U$. maydis. The cloned $E$. coli was grown on selective medium. PCR amplification and sequencing confirmed the correct replacement of the gene um03848 in yeast and E. coli.

Table A. 3 Primers used in Appendix II.

\begin{tabular}{ll}
\hline Primers & Sequence \\
\hline um3848ovFw BamHI & acaacatcatccacgGGATCCGCTGAAACGTAAAGCGAACC \\
um3848cR-BamHI & cggccgttactagtggatccGCCTCACATTGCCTACAACC \\
GalF & aatatacctctatacttaacgtc \\
TOPOT.3848Fw & taacggccgccagtgtgctgGCTCCATCGGAGAAATGAAGT \\
TOPOT.3848R & cagtgtgatggatatctgcaGCTGAAACGTAAAGCGAACC \\
M13 Rev & CAGGAAACAGCTATGAC \\
um03848qRTLft & TCTGGCTGCGTTCGTGAGT \\
um03848qRTRt & TGGTGGCGATAAGTCCAAAGT \\
\hline
\end{tabular}

Permeabilization of Cells. The yeast cells were permeabilized by using BioVision Mitochondria Isolation Kit (BioVision Incorporated) by following up till the permeabilization steps. The lysis of cell was confirmed with $30-40 \%$ decrease in $\mathrm{OD}_{600}$ after treatment with lysis enzyme. E. coli cells were permeabilized by suspending collected pellet in $10 \mathrm{mM}$ Tris Buffer $\mathrm{pH} 8.0,1 \mathrm{mg} / \mathrm{mL}$ lysozyme (ThermoFisher Scientific) and $10 \mu \mathrm{L} / \mathrm{mL}$ of proteinase inhibitor cocktail (BioVision Incorporated). Cells 
were incubated at $4{ }^{\circ} \mathrm{C}$ on rotary shaker at $250 \mathrm{rpm}$ for 30 minutes. Both yeast and bacterial crude extract were store at $-80{ }^{\circ} \mathrm{C}$ until further use.

Detection of protein. Proteins were separated in SDS-PAGE for which Mini-Protean TGX (Tris-Glycine eXtended) Precast Gels (BIO-RAD) were used. For the size-based separation of polypeptides on SDS-PAGE, the protein was denatured by mixing with $2 \mathrm{X}$ Laemmli-running buffer (BIO-RAD) containing SDS at 1:1 ratio and boiling at $80{ }^{\circ} \mathrm{C}$ for 3 min. $50 \mu \mathrm{L}$ of this mix was loaded into gel. Pre-stained protein PageRuler (BioLabs) of MW range 10 to $250 \mathrm{kDa}$ was used as a standard to identify the presence of our protein (predicted size $66.5 \mathrm{kDa}$ ). The gel was run at constant $\mathrm{mA}$ of 20-30 for 30 minutes or until protein standard was well separated or the lowest band reached to the end of the gel. The gel was dyed with Coomassie stain overnight at room temperature. The stained gel was treated with Coomassie destaining solution at room temperature for 30 minutes. The gel was observed under white light to detect the protein of interest.

Enzyme activity. Nitrite reductase activity was assayed in permeabilized yeast and bacteria cells as a function of the depletion of the substrate (nitrite) via colorimetric method as described previously ${ }^{142}$. Aliquots $(250 \mu \mathrm{L})$ of the permeabilized yeast or bacteria cells was mixed with $50 \mathrm{mM}$ phosphate buffer, $\mathrm{pH} 7.0$ and $0.1 \mathrm{mM} \mathrm{NaNO}_{2}$ in total reaction volume of $500 \mu \mathrm{L}$. The reaction mix was incubated at $30{ }^{\circ} \mathrm{C}$ with gentle shaking for one hour. The enzymatic reaction was terminated by the addition of $250 \mu \mathrm{L}$ of $1.0 \mathrm{M}$ zinc acetate and $250 \mu \mathrm{L}$ of $1 \%$ sulfanilamide in $50 \%$ TCA (trichloroacetic acid) and cooling on ice for 5 minutes. Color development was carried out by adding $250 \mu \mathrm{L}$ of $\mathrm{N}(1-$-naphthyl) ethylenediamine (NED) and incubation at room temperature for 30 minutes. A tube containing substrate without enzyme and a tube with enzyme minus 
substrate were used as control. The optical density (OD) was read for all samples at 540 $\mathrm{nm}$ in a spectrophotometer. This is the standard colorimetric method to measure the color intensity of the azo dye formed by the coupling of diazotized sulfanilamide with NED and the nitrite in the reaction mixture.

\section{Results}

\section{In silico analysis of um 03848 protein predicted localization as in cytoplasm. The}

prediction tools for the sub-cellular localization of proteins such as TargetP

(http://www.cbs.dtu.dk/services/TargetP/) and SignalP

(http://www.cbs.dtu.dk/services/SignalP/) suggested that the um03848 is a cytoplasmic protein (Figure A.3 A and B)

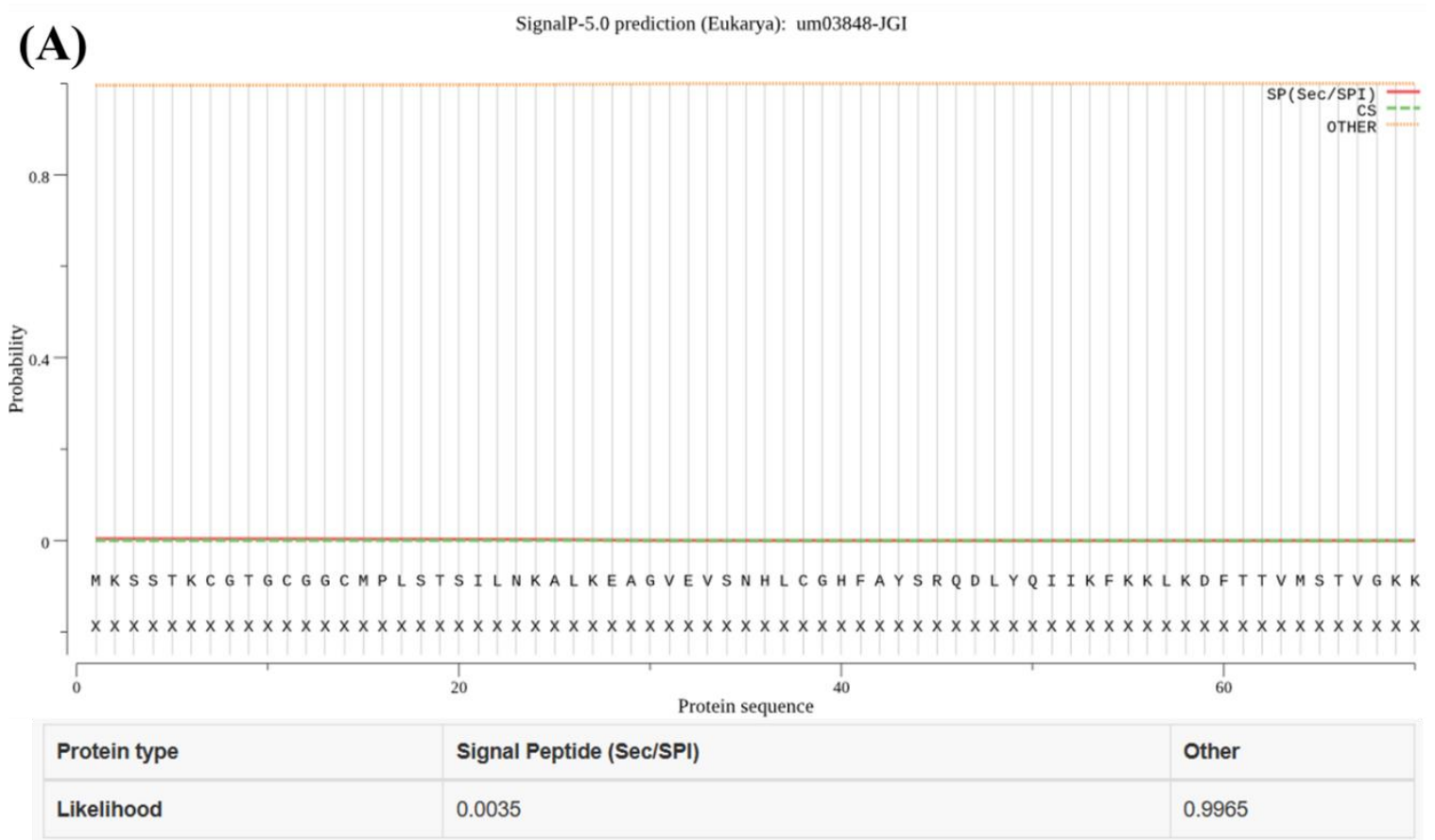




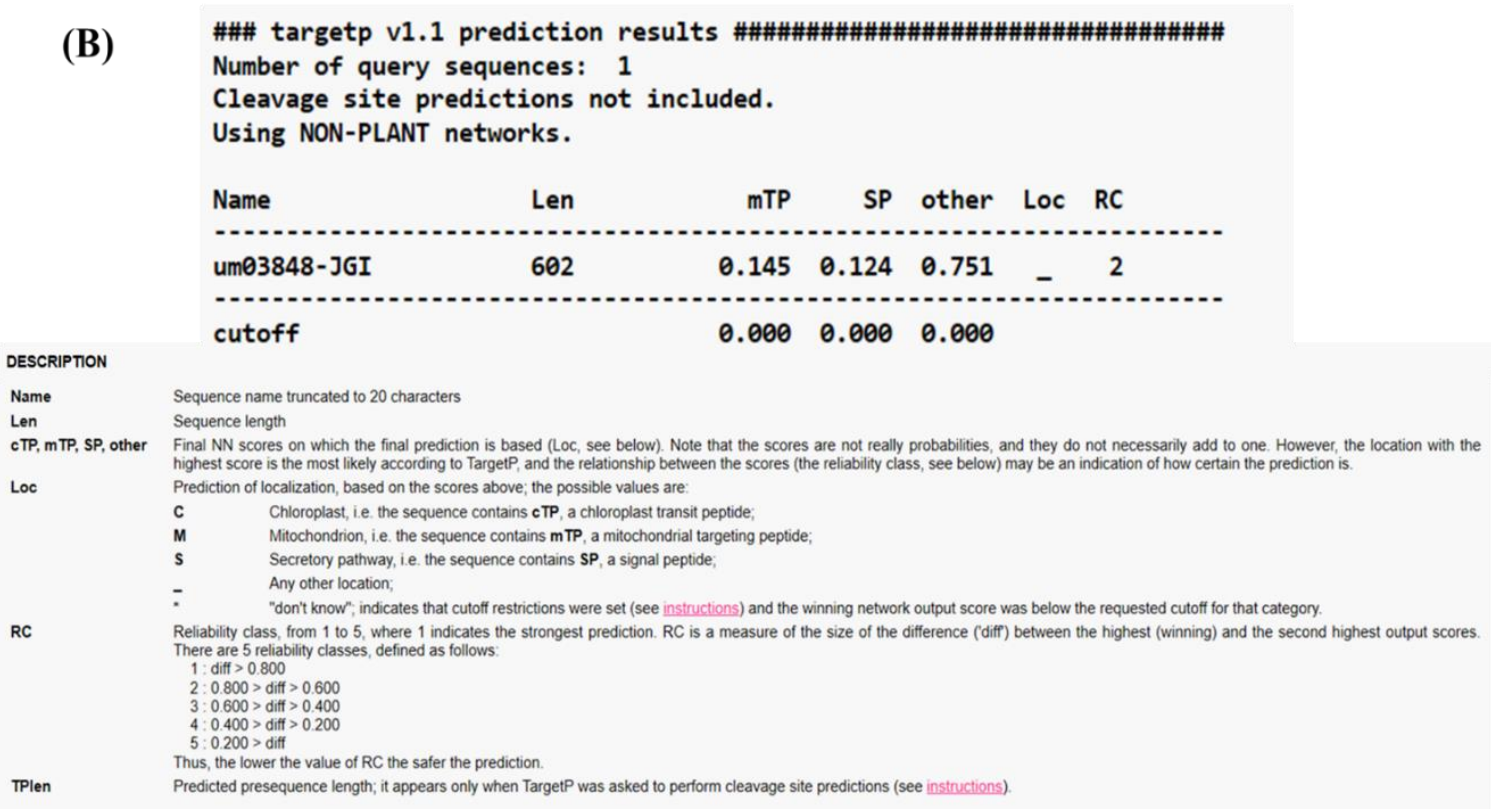

Figure A. 3 Prediction of localization of um03848 protein.

(A) SignalP 5.0 prediction of the presence of signal peptide. SignalP did not find any signal peptide sequence in um03848 protein (shown by likelihood of $<0.005$ ) (B) TargetP prediction of sub-cellular localization of um03848. Table indicated that um03848 is not a secreted protein (shown by low SP value) and may have a sub-cellular location other (high value in other) than mitochondria (low mTP value) and chloroplast (no cTP value).

\section{PCR amplification showed the correct orientation of um03848 in transformed cells.}

To know the correct orientation of um03848 in transformed S. cerevisiae and E. coli, plasmid isolated from each strain were PCR amplified. For yeast, um03848 should be expressed under Gal promoter so um03848 will be at 3' end of Gal promoter. To detect this, GalF primer combined with 3848qRTLft and 3848qRTRt was used. Correct orientation of $u m 03848$ was shown by $\sim 1400$ bp by GalF - 3848qRTRt primers in $1 \%$ agarose gel (Figure A.4 A). For bacteria, M13 Rev primer was used in combination with TOPOT and $\mathrm{qRT}$ primers as listed on table A.3. The $\sim 2 \mathrm{~kb}$ and $\sim 900 \mathrm{bp}$ bands shown by 
primers M13R - TOPOT.3848R and M13R - 3848qRTRt respectively (Figure A.4 B) indicates the correct orientation of $u m 03848$ in transformed $E$. coli. Furthermore, this was also confirmed by nucleotide sequencing.

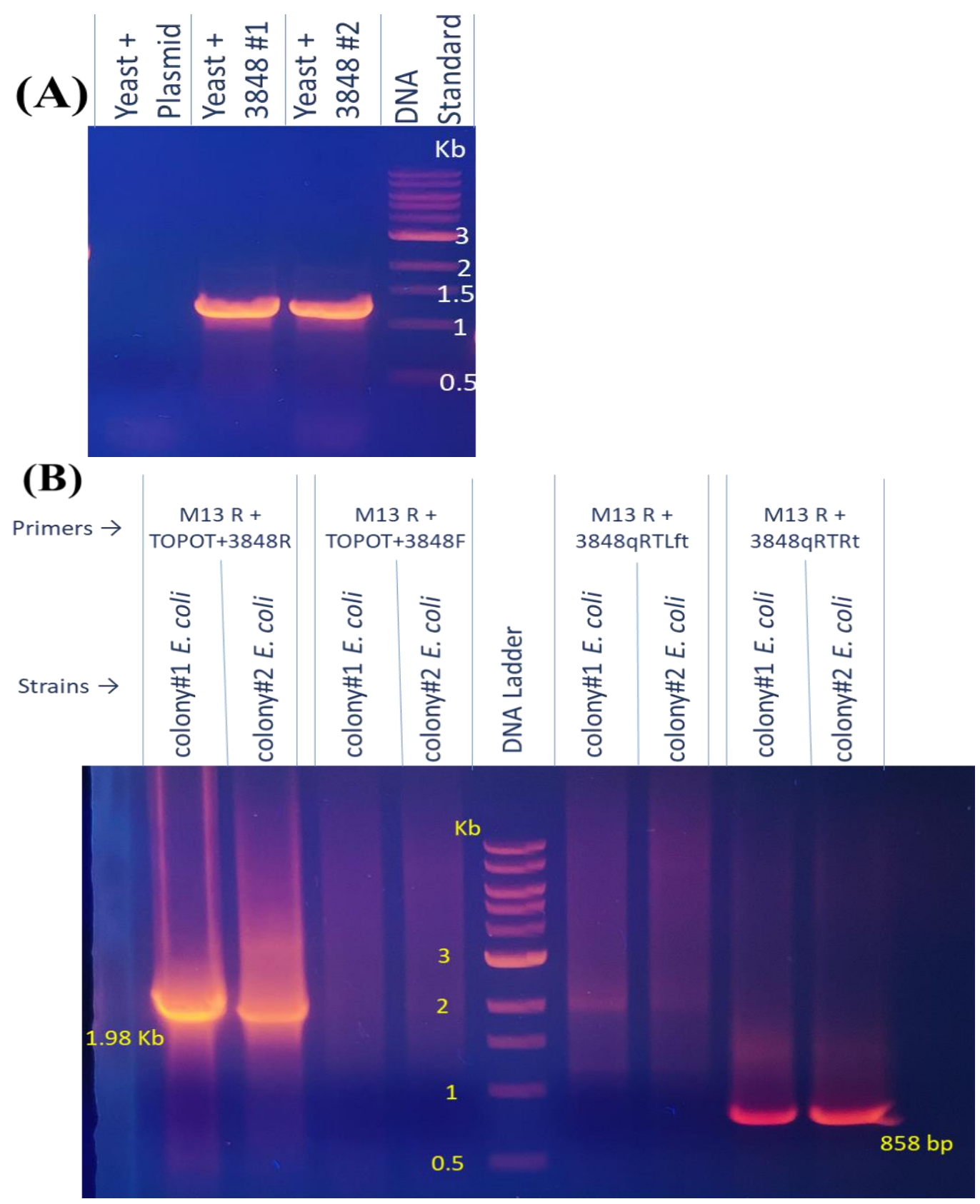

Figure A. 4 Agarose gel electrophoresis to determine the orientation of um03848 in transformed cells. 
The PCR amplified products on the plasmid isolated from transformed (A) S. cerevisiae and (B) E. coli cells was ran in 1\% agarose gel. The set of primers used are shown on the top. The bands indicated in the panels showed the correct orientation of the gene.

Presence of um03848 transcript in transformed yeast (MLY40a Ura-) cells. The total RNA was extracted from galactose induced transformed yeast (MLY40 $\alpha \mathrm{Ura}^{-}$) strains.

The strains include yeast + plasmid (MLY40 $\alpha \mathrm{Ura}^{-}+$pYES2.1TOPO). and yeast +3848 $\left(\right.$ MLY40 $\alpha \mathrm{Ura}^{-}+$pYES2.1TOPO + 3848). qRT-PCR performed on total RNA extracted showed the 10-fold expression of um03848 in transformed yeast cells (Figure A.5).

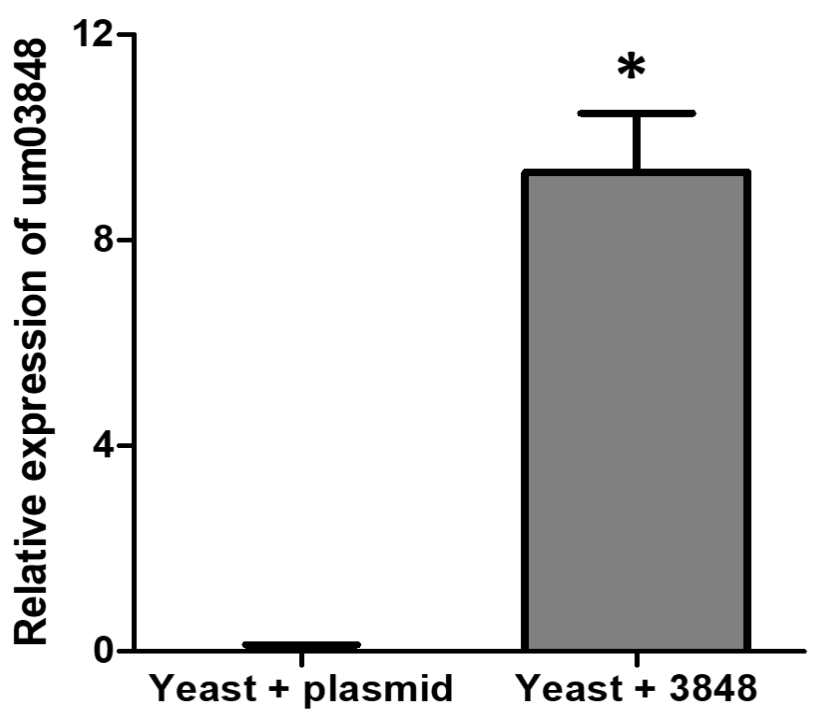

Figure A. 5 Relative expression of um03848 in transformed S. cerevisiae. The fold changes in expression of the strains induced by galactose is shown. Bar represents the averages of duplicate and standard errors are indicated in the graphs. Unpaired Student's t-test was performed in GraphPad Prism, comparing with yeast + plasmid. P-value $<0.05$ is considered as significant. * indicates the significant difference in the expression level of target gene for the yeast strain with um03848 gene with respect to the yeast with vector but without um03848.

\section{SDS-PAGE protein separation didn't detect um03848 protein in transformed}

mutants. The crude extract from E. coli and S. cerevisiae was run in a SDS-PAGE 
(sodium dodecyl sulfate polyacrylamide gel electrophoresis) in which proteins are separated in relative to molecular mass ${ }^{223,224}$. The $1 \mathrm{mg} / \mathrm{mL}$ BSA (Bovine Serum Albumin) was also ran for the control. There was no clear band (Figure A.6) for the predicted size of um03848 (66.5 kDa).

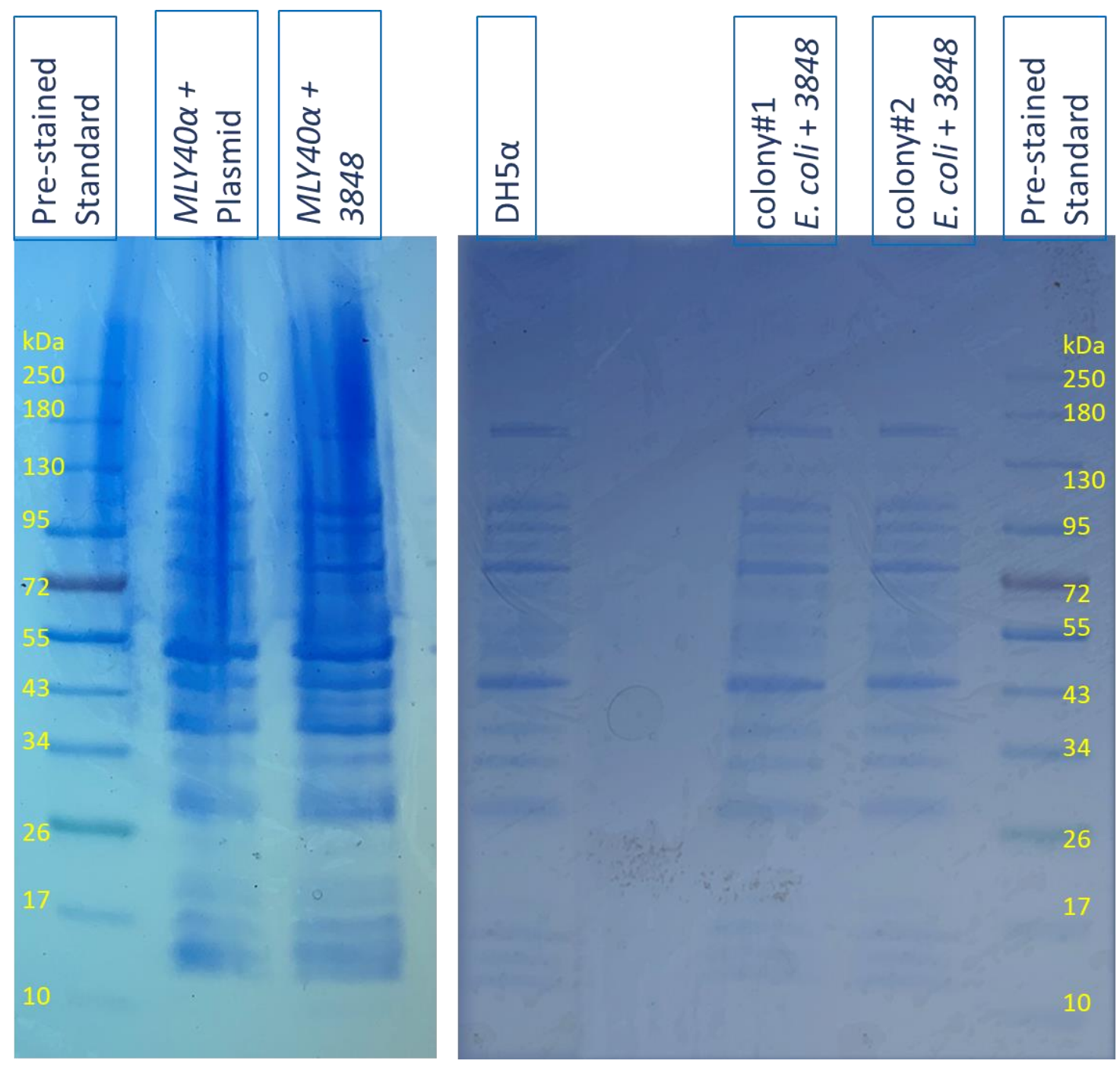

Figure A. 6 SDS-PAGE to separate proteins present in crude extract of transformed $S$. cerevisiae and E. coli.

The SDS buffer was added in 1:1 ratio to the crude extract obtained from each cell typeyeast transformed with $u m 03848$ gene (MLY40 $\alpha+3848$ ), yeast transformed with plasmid only (MLY40 $\alpha+$ plasmid), competent $E$. coli (DH5 $\alpha$ ) and E. coli cloned with vector containing um03848. The mix was boiled at $80{ }^{\circ} \mathrm{C}$ for $3 \mathrm{~m}$ and $50 \mu \mathrm{L}$ loaded to separate well of SDS-PAGE. Pre-stained Page-Ruler as a size standard was loaded to one 
well. PAGE observed after staining and destaining treatment showed no difference in protein profile between the transformed and non-transformed cells.

Furthermore, the crude extract was used for the nitrite reductase enzyme activity assay following the procedure explained in the methods section. There was a difference between the color intensity developed by negative control (tube with substrate but no enzyme) and the crude extract, with crude extract having lower color intensity. However, there was no detectable difference in the color intensity between transformed vs untransformed E. coli or S. cerevisiae from spectrophotometer readings (data not shown). This indicates that there is something in crude extract that reduced the color intensity but the nitrite reductase activity of $u m 03848$ is not detectable. However, due to time limitation the protocol for the biochemical assay could not be optimized based on laboratory conditions.

\section{Discussion}

Nitrite reductase was first identified by Losada and Peneque as an enzyme catalyzing ${ }^{225}$. Since then, the enzyme has been purified and studied in different plants, cyanobacteria, algae and yeast ${ }^{52,53,220,226}$. In terms of filamentous fungi, Aspergillus nidulans and Neurospora crassa have been mostly studied. However, the nitrite reductase experiments on these fungi are mostly in gene mutational level than protein biochemistry 107,146. Per kilogram fresh weight of N. crassa, is expected to have $20 \mathrm{mg}$ of nitrite reductase ${ }^{227}$. Therefore, it is difficult to study the enzymatic action of the nitrite reductase enzyme inside fungal system. Besides, the purification or the isolation of nitrite reductase by pull-down assay is also not possible because of lack of anti-nitrite reductase 
antibodies. Therefore, the inducible promoter systems based on available carbon and nitrogen sources have been used to follow the localization or fate of protein. In this chapter, we attempt to express the nitrite reductase under the induction of galactose promoter in yeast system. We also express the enzyme in E. coli system as the generation time is faster and easy to manipulate in bacteria than fungi.

Although we were able to see the correct orientation of the gene and expression on transcript level in yeast and bacterial system, we couldn't identify um03848 protein in SDS-PAGE. In addition, the enzyme assay didn't give any indication of the nitrite reductase activity in both systems tested. The nitrite reductase is high energy consuming enzyme. Therefore, gene expression and protein translation of nitrite reductase is highly regulated ${ }^{162}$. Because of the complexity of the enzyme, the biochemical study in nitrite reductase is limited. The filamentous fungi to which nitrite reductase in U. maydis, um03848, showed protein sequence similarity (Table 2.4 in Chapter II), they were identified based on bioinformatics and mutational analyses but not through biochemical analysis. Most of the biochemical analyses of nitrite reductase is done in yeast and bacteria.

Purified nitrite reductase in yeast Candida utilis is a heterodimer of sizes $66 \mathrm{kDa}$ and $58 \mathrm{kDa}$ where both the subunits can catalyze nitrite reduction ${ }^{228}$. In addition, each of the subunits of nitrite reductase in $C$. utilis undergoes phosphorylation dephosphorylation reaction differentially with the regulatory significance. For example, when cells are grown in nitrate in vitro, the $66 \mathrm{kDa}$ subunit is highly phosphorylated while the $58 \mathrm{kDa}$ subunit is not and this increases nitrite reductase enzyme activity ${ }^{229}$. Besides, nitrite reductase in fungi and bacteria uses $\mathrm{NAD}(\mathrm{P}) \mathrm{H}$, while plants and algae use 
ferredoxin. Nitrite reductase in plants and some fungi reduces nitrite into nitric oxide while in the assimilatory system, nitrite reductase is involved in assimilative metabolism where nitrite is directly reduced to ammonia ${ }^{230-232}$. This indicates the complexity in the structure and regulation of the nitrite reductase enzyme. Therefore, unraveling the property and structure of nitrite reductase in filamentous fungi will be helpful to understand the activity of the enzyme. Tagging proteins of interest will be helpful to purify with affinity or size-exclusion chromatography. With this, the functional/ biochemical property of the proteins can be studied better. Furthermore, optimization of the enzymatic assay for the specific cells and specific conditions is required. 


\section{APPENDIX III \\ ROLE OF DELETION OF UM00037 GENE IN FB1 AND FB2 STRAINS TO THE VIRULENCE OF U. MAYDIS}

\section{Summary}

This study was conducted to understand the role of the um00037 gene in $U$. maydis. This gene was selected because in the analysis of amino acid sequence in different bioinformatic tools such as InterPro, Pfam and PROSITE, it showed a sequence similarity to the nitrite reductase domain, Rieske and oxidoreductase domains. To evaluate the role of um00037 in mating of $U$. maydis and in the virulence to its host plant, the um00037 gene was disrupted in mating compatible haploid strains FB1 and FB2. The phenotypes of the um00037 deletion mutants regarding mating efficiency and pathogenesis were examined. The result showed that the deletion of um00037 in $U$. maydis did not affect mating with its compatible partner, as assessed by "fuzz" in the charcoal mating assay. Also, the deletion of um00037 in U. maydis does not affect its virulence in maize.

\section{Methods}

The U. maydis strains used in this study are listed in Table A.4 below. Strains were grown at $27{ }^{\circ} \mathrm{C}$ in solid or liquid YEPS. Mating assay was done by co-spotting premixed compatible haploid strains in solid PDA with $1 \%$ activated charcoal plate as mentioned previously in Chapter II. For plant infection assay, strains grown in $20 \mathrm{ml}$ YEPS media up to $\mathrm{OD}_{600}$ of 1.0 were collected and resuspended in water to concentrate up to $\mathrm{OD}_{600}$ 3.0. The 8 days old Golden Bantam corn seedlings [Organic Golden Bantam, 
Territorial Seed Company] was injected with pre-mixed haploid strains of opposite mating type. The pathogenicity symptoms were measured from $0-5$ disease index ${ }^{78}$ as mentioned earlier in Chapter II. Statistical analysis of the disease index measures was performed using a Kruskal-Wallis Test with Multiple Comparison Test using R program 119,120 .

Table A. 4 List of strains used in Appendix III.

\begin{tabular}{|c|c|c|}
\hline Ustilago Strain & Genotype & Reference \\
\hline FB1 & $a l(m f a l$ pral) bl (bEl bWl) & \multirow{2}{*}{$\begin{array}{l}\text { Banuett and } \\
\text { Herskowitz, } 1989\end{array}$} \\
\hline FB2 & $a 2$ (mfa2 pra2) b2 (bE2 bW2) & \\
\hline FB1 $\Delta u m 00037$ & alb1 um00037::hyg ${ }^{R}$ & This study \\
\hline FB2 $\Delta u m 00037$ & $a 2 b 2$ um00037::hyg $g^{R}$ & This study \\
\hline
\end{tabular}

\section{Results}

To perform the in silico analysis of um00037 gene, at first, the um00037 nucleotide sequence was obtained from Joint Genome Institute, JGI (https://genome.jgi.doe.gov/pages/search-for-genes.jsf?organism=Ustma1). Secondly, the nucleotide sequence was translated into amino acid in Expasy-translate tool at https://web.expasy.org/translate/. Third, the translated amino acid sequence was characterized in NCBI conserved domain analysis tool at https://www.ncbi.nlm.nih.gov/Structure/cdd/wrpsb.cgi. The um00037 protein has sequence similarity to the large domain of NiRB superfamily. It also had hits with Pyridine nucleotide-disulphide oxidoreductase family, 2Fe-2S cluster binding site and and Rieske domain (Figure A.7). 


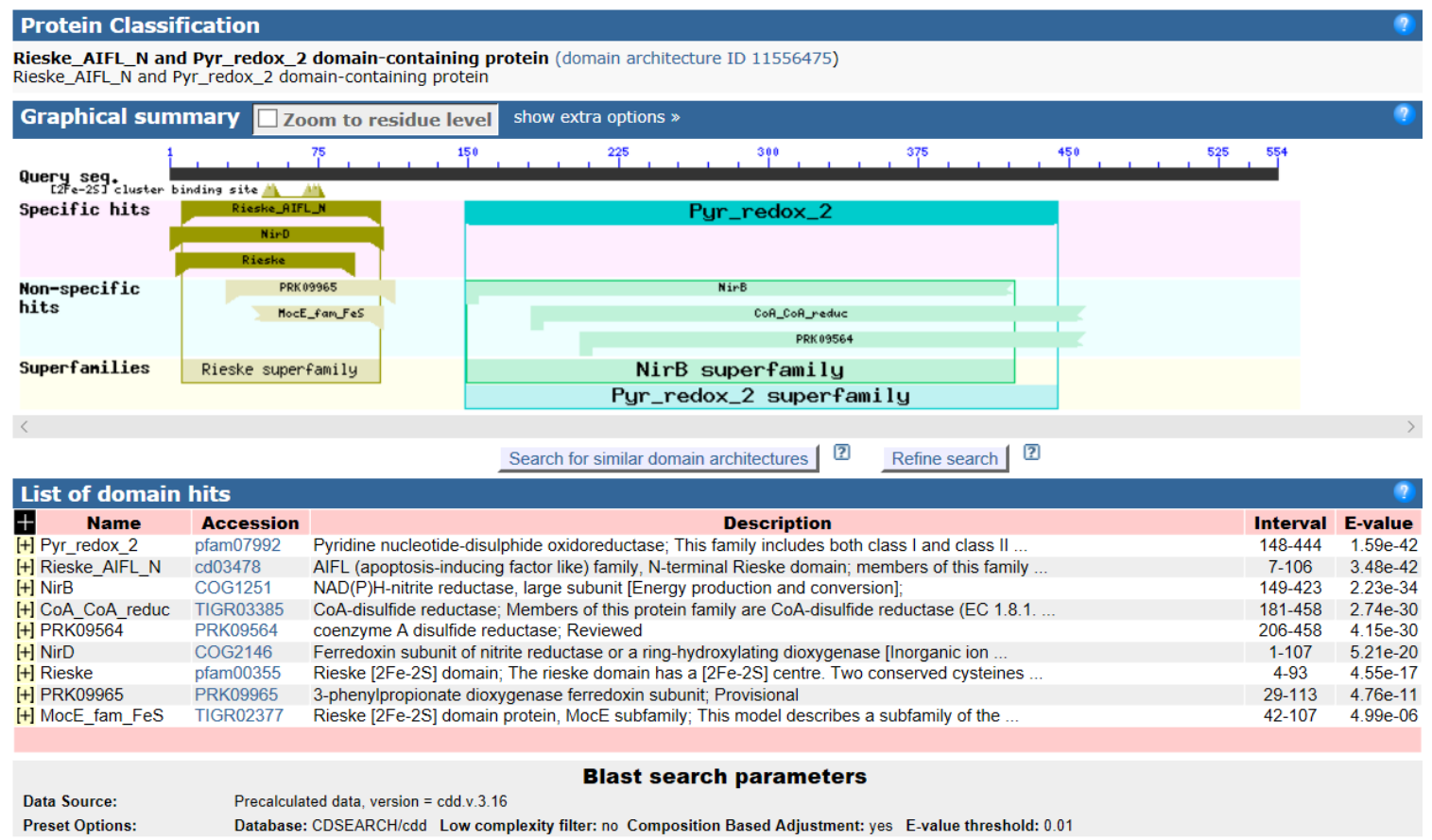

Figure A. 7 Conserved Domain Analysis for um00037 amino acid sequence.

The graphical summary indicates the presence of different conserved domains in different regions of the query um03848 sequence (black). The table gives the list of domain hits and the description of domain.

The mating assay was performed on PDA charcoal medium for um00037 deleted

FB1 and FB2 mutants along with mating compatible wild type haploids (FB1 X FB2).

The compatible mating strains of $\Delta u m 00037$ showed similar dikaryotic white "fuzz" as of wild type strains (Figure A.8 A). This depicts that the deletion of um00037 in FB1 and FB2 background doesn't affect the mating efficiency of $U$. maydis.

To determine the effects of these mutants on virulence to the host plant, the opposite haploid strains grown overnight were pre-mixed and injected into 7-days-old maize seedlings. Disease symptoms were scored at 14 days post infection (dpi) with the infection ranking of 0 to 5 as mentioned earlier. The percentage of plants with specific symptoms of infection is shown in Figure A.8 B. The infections caused by the pre-mixed 
compatible haploid strains of $\Delta u m 00037$ did not show any significant difference in plant virulence compared to the wild type strains. Therefore, gene um00037 is not involved in pathogenicity to the host plant.

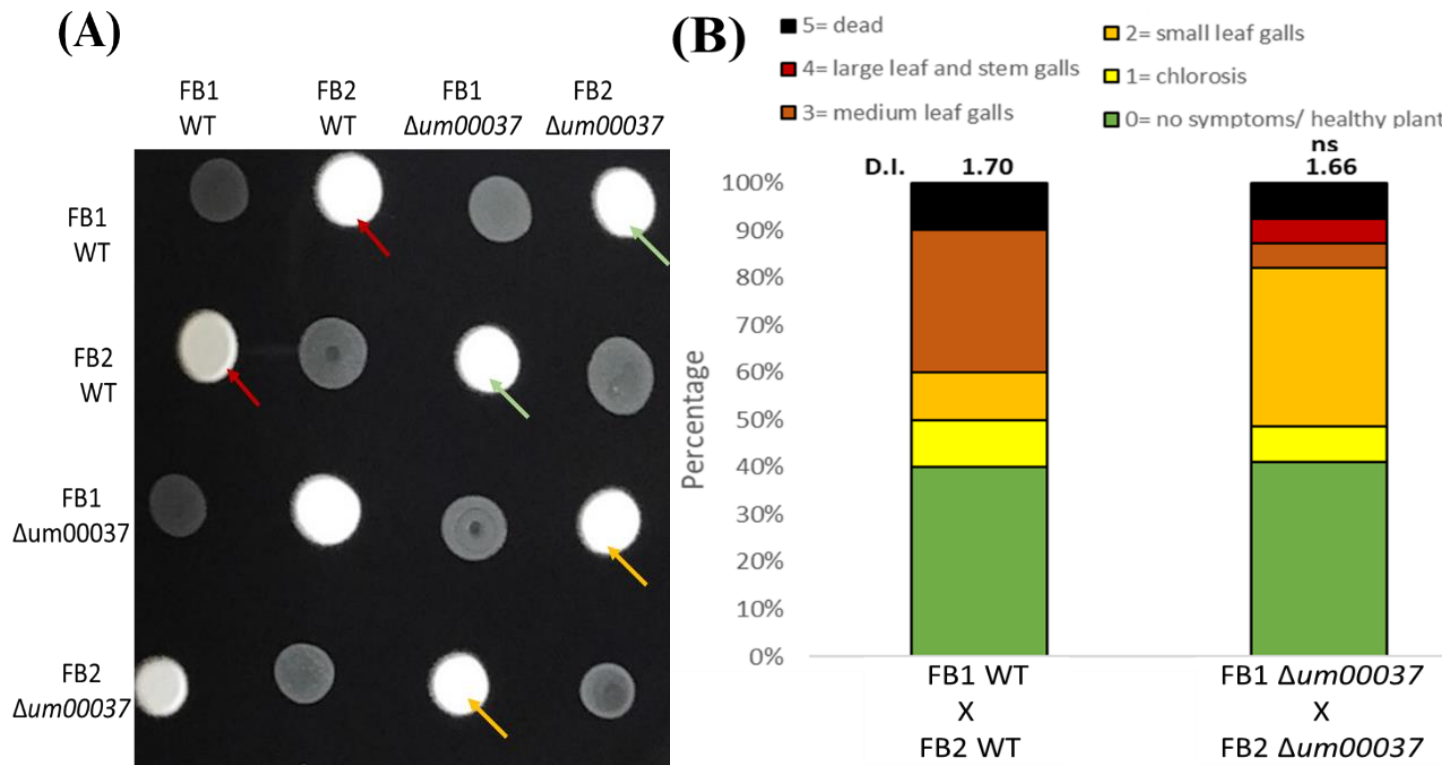

Figure A. 8 Mating and pathogenicity by $\Delta$ um00037 in U. maydis.

(A) Mating assay on PDA charcoal medium where equal mixtures of opposite mating wild type strains (FB1 X FB2), corresponding $\Delta u m 00037$ (FB1 X FB2) and the mutants with the opposite wild type strains were co-spotted. After $24 \mathrm{~h}$ incubation, mating reaction was examined by the presence of white "fuzz" phenotype of aerial hyphae production. Red and yellow arrows indicate the successful mating in wild type and $\Delta u m 00037$ respectively. Green arrows show the mating of $\Delta u m 00037$ with its opposite wild type strains (B) Pathogenicity assay where disease symptoms were scored at 14 dpi in the categories of 0 to 5 as depicted at the top of the figure. Graph shows the percentage of plants with specific symptoms of infection. The $\mathrm{X}$-axis indicates the paired background that is injected into maize plant. The data was analyzed using Kruskal-Wallis test followed by post-hoc comparison. The infection by $\Delta u m 00037$ was not significantly different (ns), p-value $>0.05$, compared to the wild type. 
CONFIRMATION OF MUTANTS BY qRT-PCR ANALYSIS

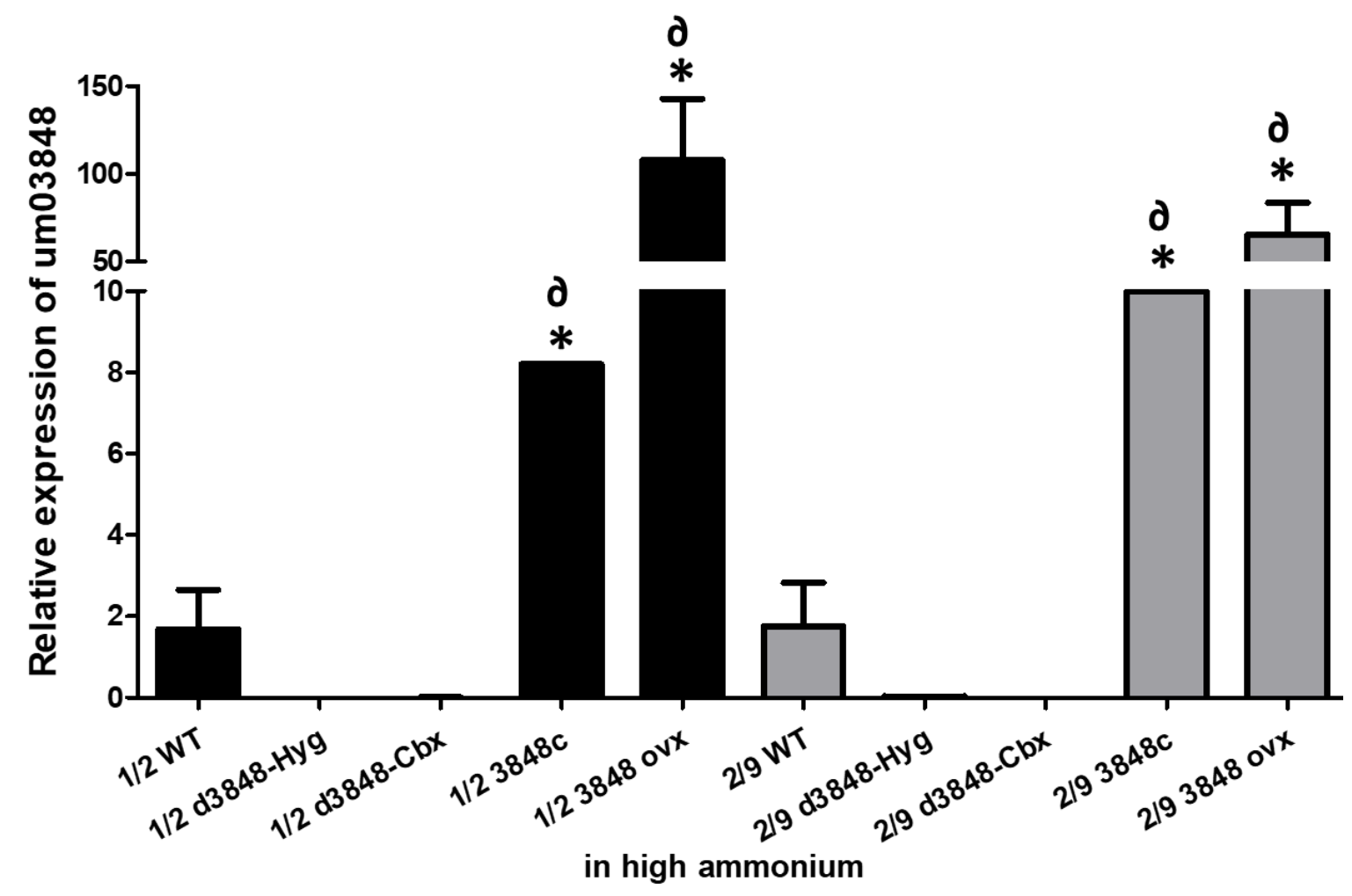

Figure A. 9 Relative expression of um03848 in mutants of $U$. maydis.

Figure represents the fold changes in expression of $u m 03848$ gene in the wild type and mutant strains (deletion, complementation and overexpression) grown on high ammonium condition. Bar represents the averages of duplicate and standard errors are indicated in the graph. One-way ANOVA followed by Tukey's comparison was performed in GraphPad Prism. P-value $<0.05$ is considered as significant. * indicates the significant difference in the um03848 expression level for mutants comparing with respective wild type i.e., mutants in $1 / 2$ mating strain was compared with $1 / 2 \mathrm{WT}$ and mutants in 2/9 was compared with 2/9 WT. $\partial$ indicates the significance in expression for the complementation and overexpression mutants comparing with expression in $\Delta u m 03848$-HygR. No expression of um03848 in d3848-Hyg and d3848-Cbx mutants on either mating background confirmed the um03848 knock-out mutants. 


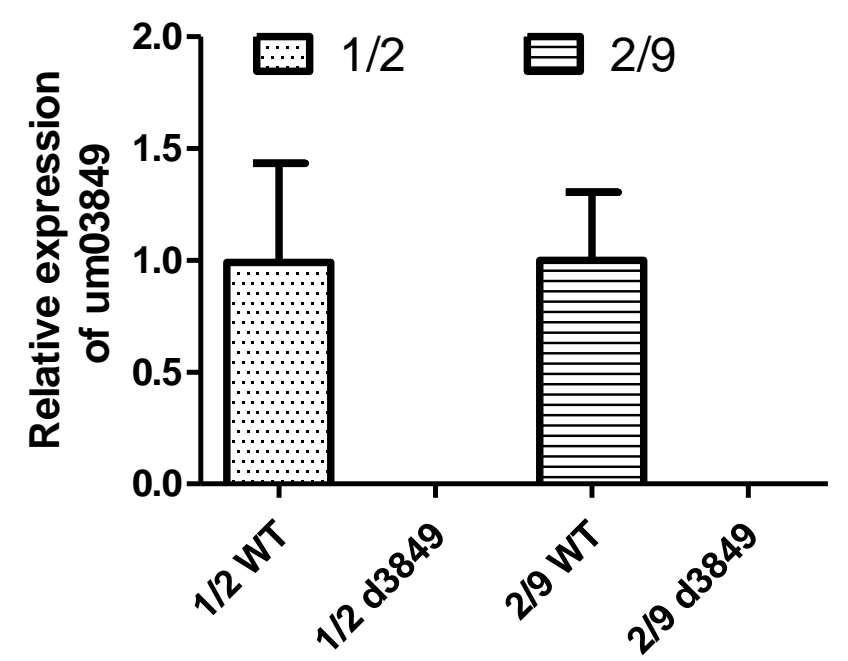

Figure A. 10 Relative expression of um03849 in mutants in U. maydis.

Figure represents the fold changes in expression of um03849 gene in the wild type and deletion strains grown on high ammonium condition. Bar represents the averages of triplicates and standard errors are indicated in the graph. Unpaired student's t-test was performed in GraphPad Prism. P-value $<0.05$ is considered as significant compared to respective wild type. No expression of um03849 in mutants on either mating background confirmed the um03849 knock-out mutants.

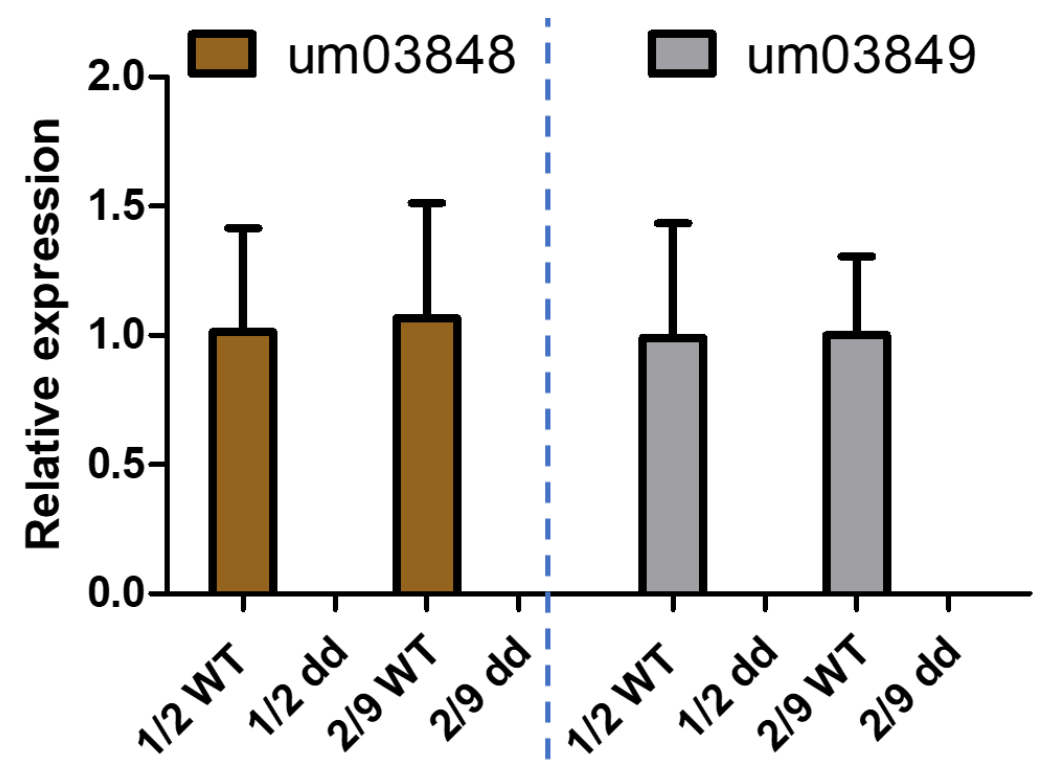

Figure A. 11 Relative expression of um03848 and um03849 in mutants in U. maydis. Fold changes in expression of um03849 gene in the wild type and deletion strains grown on high ammonium condition is shown. Bar represents the averages of triplicates and standard errors are indicated in the graph. No expression of um03848 and um03849 confirmed the double deletion of these genes in mutants. 


\section{CURRICULUM VITAE}

NAME: $\quad$ Sunita Khanal

ADDRESS: $\quad 741$ E Madison St.,

Louisville, KY 40202

DOB: $\quad$ Kathmandu, Nepal - October 12, 1987

PHONE: $\quad$ 502-715-5572

EMAIL: $\quad$ sunitakhanal7@gmail.com

EDUCATION: Tribhuvan University, Nepal Bachelor of Science: Microbiology

2006-10

Tribhuvan University, Nepal

Master of Science: Biotechnology

2010-13

University of Louisville, USA

Master of Science: Biology

2015-17

University of Louisville, USA

Doctor of Physiology in Biology: Mol., Cel and Dev. Program 2015-20

\section{PUBLICATIONS:}

S. Khanal, R. Malla, S. Shrestha (2013) Exploration of soil fungal diversity of Manaslu Conservation Area (MCA), Nepal and biotechnological and molecular characterization of selected species. M.Sc. in Biotechnology. Thesis. Tribhuvan University, Nepal. 
Lok Ranjan Bhatt, Sunita Khanal, Agni Raj Koirala and Hem Raj Pant (2019) Preparation and surface morphology of herbal based polylactide microspheres. Materials letter. 235 (15). Pp 157-160.

Sunita Khanal and M. H. Perlin. Enzyme activity of U. maydis nitrite reductase, um03848. (Manuscript under preparation).

Sunita Khanal, Kiara Smith, Alejandro Nava, Luke Schroeder and Michael H. Perlin. Role of Ustilago maydis nitrate assimilatory genes in mating and virulence. (Manuscript under preparation).

\section{PRESENTATIONS:}

Sunita Khanal, Kiara Smith and Michael H. Perlin. Role of Ustilago maydis nitrite reductase, um03848, in mating and virulence. $30^{\text {th }}$ Fungal Genetics Conference, Asilomar, CA, March 2019 (Poster presentation).

Sunita Khanal, Kiara Smith, Alejandro Nava and Michael H. Perlin. Role of Ustilago maydis nitrite reductase, um03848, in mating and virulence. $2^{\text {nd }}$ Ustilago-Smut convergence, March 2019 (Oral presentation).

\section{TEACHING EXPERIENCE:}

Graduate Teaching Assistant, Biology Department, University of Louisville, KY Laboratory for Introduction to Biological Systems - SL (BIOL 104)

Fall 2015

Spring 2016

Summer 2016

Fall 2017

Spring 2018

Principles of Biology Laboratory (BIOL 244)

Summer 2018

Fall 2018

Spring 2019

Summer 2019

Fall 2019

Spring 2020

\section{COMMUNITY SERVICES:}

Treasurer. Nepalese Student Association-University of Louisville. 
August 2017 - July 2018

Judge for Senior Biology and Health Science. Louisville Regional Science and Engineering Fair (LRSEF).

March 2018 and 2019

Reviewer for Nepal Journal of Biotechnology (NJB).

2018 - Present

\section{AWARDS/ GRANTS:}

Arts \& Sciences Research \& Creative Activities Grant.

University of Louisville

April 2016

GNAS Research Grant

University of Louisville

September 2016 and October 2017

Fungal Conference Travel Award

Genetics Society of America

November 2018

GNAS Travel Grant

University of Louisville

January 2019

BGSA Grant Award

University of Louisville

April 2019

Undergraduate Mentor-Mentee Research Award

Arts \& Science Program

University of Louisville

September 2019 - March 2020

Graduate Teaching Assistantship

University of Louisville

August 2015- July 2020 